\title{
Uncertainty Propagation through Dependability Models
}

by

Kesari Mishra

Department of Electrical and Computer Engineering

Duke University

Date:

\author{
Approved:
}

Kishor S. Trivedi, Supervisor

\begin{tabular}{c}
\hline John A. Board \\
\hline Krishnendu Chakrabarty
\end{tabular}

Benjamin C. Lee

Henri P. Gavin

Dissertation submitted in partial fulfillment of the requirements for the degree of Doctor of Philosophy in the Department of Electrical and Computer Engineering in the Graduate School of Duke University

2011 


\section{ABSTRACT \\ (Electrical and Computer Engineering) \\ Uncertainty Propagation through Dependability Models \\ by}

Kesari Mishra

Department of Electrical and Computer Engineering

Duke University

Date:

Approved:

\begin{tabular}{c}
\hline Kishor S. Trivedi, Supervisor \\
\hline John A. Board \\
\hline Krishnendu Chakrabarty \\
\hline Benjamin C. Lee \\
\hline Henri P. Gavin
\end{tabular}

An abstract of a dissertation submitted in partial fulfillment of the requirements for the degree of Doctor of Philosophy in the Department of Electrical and Computer Engineering

in the Graduate School of Duke University

2011 
Copyright (c) 2011 by Kesari Mishra

All rights reserved except the rights granted by the Creative Commons Attribution-Noncommercial Licence 


\section{Abstract}

Systems in critical applications employ various hardware and software fault-tolerance techniques to ensure high dependability. Stochastic models are often used to analyze the dependability of these systems and assess the effectiveness of the fault-tolerance techniques employed. Measures like performance and performability of systems are also analyzed using stochastic models. These models take into account randomness in various events in the system (known as aleatory uncertainty) and are solved at fixed parameter values to obtain the measures of interest. However, in real life, the parameters of the stochastic models themselves are uncertain as they are derived from a finite (limited) number of observations or are simply based on expert opinions. Solving the stochastic models at fixed values of the model input parameters result in estimates of model output metrics which do not take into account the uncertainty in model input parameters (known as epistemic uncertainty).

In this research work, we address the computation of uncertainty in output metrics of stochastic models due to epistemic uncertainty in model input parameters, with a focus on dependability and performance models of current computer and communication systems. We develop an approach for propagation of epistemic uncertainty in input parameters through stochastic dependability and performance models of varying complexity, to compute the uncertainty in the model output measures. The uncertainty propagation method can be applied to a wide range of stochastic model types with different model output measures. 
For simple analytic stochastic dependability models, we present a closed-form analytic method for epistemic uncertainty propagation, where we derive closed-form expressions for the expectation, distribution and variance of the model output metrics due to the epistemic uncertainty in the model input parameters. We analyze the results thus obtained and study their limiting behavior. For slightly more complex analytic stochastic models, where the closed-form expressions for the expectation, distribution and variance of the model output cannot be easily obtained, we present a numerical integration based method. For large and complex stochastic models, we develop a sampling based epistemic uncertainty propagation method which also considers dependencies in the input parameter values and is an improvement over previous sampling based uncertainty propagation approaches. The sampling based epistemic uncertainty propagation method explained in this dissertation acts as a wrapper to existing models and their solution types (hence the wide applicability) and provides more robust estimates of uncertainty in the model output metrics than previous sampling based methods.

We demonstrate the applicability of the uncertainty propagation approach by applying it to analytic stochastic dependability and performance models of computer systems, ranging from simple non-state-space models with a few input parameters to large state-space models and even hierarchical models with more than fifty input parameters. We further apply the uncertainty propagation approach to stochastic models with not only analytic or analytic-numeric solutions but also those with simulative solutions. We also consider a wide range of model output metrics including reliability and availability of computer systems, response time of a web service, capacity oriented availability of a communication system, security (probability of successful attack) of a network routing session, expected number of jobs in a queueing system with breakdown and repair of servers and call handoff probability of a cellular wireless communication cell. 


\section{Contents}

Abstract $\quad$ iv

List of Tables $\quad$ X

List of Figures $\quad$ xi

Acknowledgements xiv

1 Introduction $\quad 1$

1.1 Epistemic Uncertainty Propagation . . . . . . . . . . . . . . 2

1.2 Contribution of this Dissertation _. . . . . . . . . . . . 10

1.3 Outline of the Dissertation . . . . . . . . . . . . . . 14

2 Background $\quad 16$

2.1 Analytic Aleatory Model Types . . . . . . . . . . . . . . . . 16

2.1.1 Non State Space Models . . . . . . . . . . . . . . . . . 17

2.1.2 State Space Models . . . . . . . . . . . . . . . . . . 19

2.1.3 Hierarchical Models . . . . . . . . . . . . . . . 22

2.2 Bayes' Theorem . . . . . . . . . . . . . . . . . 23

2.2.1 Choice of Priors . . . . . . . . . . . . . . . 24

2.3 Epistemic Distributions . . . . . . . . . . . . . . . 26

2.3.1 Determining Epistemic Distributions . . . . . . . . . . . 27

2.3.2 Epistemic Distribution: Exponential Aleatory Distribution . . 28

2.3.3 Epistemic Distribution: Bernoulli Aleatory Distribution . . . . 29 
2.4 Aleatory Vs. Epistemic Independence . . . . . . . . . . . . . . . . . . 31

\section{Closed-form Approach for Epistemic Uncertainty Propagation 33}

3.1 Uncertainty Propagation . . . . . . . . . . . . . . . . . . . 33

3.2 Reliability of a Single Component System . . . . . . . . . . . . . 36

3.2.1 Distribution of Reliability . . . . . . . . . . . . 36

3.2.2 Expected Reliability _. . . . . . . . . . . . . . . 38

3.2.3 Variance of Reliability . . . . . . . . . . . . . . . 40

3.3 Series System Reliability . . . . . . . . . . . . . . . . 41

3.3.1 Comparing Confidence Interval . . . . . . . . . . . . . . . . . 42

3.4 Reliability of a TMR System . . . . . . . . . . . . . . . 43

3.4.1 Distribution of Reliability : TMR System . . . . . . . . . . . 44

3.4.2 Expected Reliability : TMR System . . . . . . . . . . . 45

3.4.3 Variance of Reliability : TMR System . . . . . . . . . . . 46

3.5 Reliability of a k-out-of-n System . . . . . . . . . . . . . . . . 47

3.5.1 Variance of Parallel System Reliability . . . . . . . . . . . . . 49

3.6 Duplex System with a Spare . . . . . . . . . . . . . 51

3.6.1 Expected Reliability : Duplex System with a Spare . . . . . . 51

3.6.2 Variance of reliability : Duplex System with a Spare . . . . 53

3.7 Reliability of Recovery Block Architecture . . . . . . . . . . . . . 55

3.8 Reliability of Composite Web Service . . . . . . . . . . . . 58

3.8.1 Comparison of Variance and Expectation of Reliability . . . . 63

3.9 Interval Availability: Cumulative Downtime Distribution Method . . 65

3.9.1 Distribution of Interval Availability . . . . . . . . . . . 66

3.9.2 Expected Interval Availability . . . . . . . . . . . . . . 67

3.9.3 Variance of Interval Availability . . . . . . . . . . . . . . . 68 
4.1 Reliability of a Composite Web Service . . . . . . . . . . . . . 71

$4.2 \mathrm{M} / \mathrm{M} / 1$ Queue with Server Breakdown and Repair . . . . . . . . . . 73

4.3 Probability of a Successful Attack . . . . . . . . . . . . . . 76

\section{Sampling Based Uncertainty Propagation $\quad 79$}

5.1 Overview of the Method . . . . . . . . . . . . . 80

5.1.1 Determining Epistemic Distributions . . . . . . . . . . 83

5.1 .2 Determining the Sample Size . . . . . . . . . . . . . 87

5.1.3 Sampling Procedure ................. 89

5.1.4 Analytic Model Solution . . . . . . . . . . . . . . . 90

5.1.5 Summarizing the Output ............... 91

5.2 Illustrative Examples . . . . . . . . . . . . . . . . . . . . . . 91

5.2.1 Availability of IBM Bladecenter Server System . . . . . . . . . 92

5.2.2 Availability Model of IBM SIP SLEE Server Cluster System . 97

5.2.3 Reliability of Remote Exploration and Experimentation System 103

5.2.4 Mean Response Time and Reliability of Composite Web Service111

6 Latin Hypercube Sampling $\quad 118$

6.1 Uncertainty Propagation with LHS . . . . . . . . . . . . . . 119

6.2 Illustrative Examples . . . . . . . . . . . . . . . . . . 121

6.2.1 Availability of IBM SIP Server Cluster System . . . . . . . . . 122

6.2.2 Reliability of Remote Exploration and Experimentation System 125

6.2.3 Reliability and mean response time of composite web service . 128

6.2.4 Capacity Oriented Availability of CMTS System . . . . . . . . 132

6.2.5 Fixed-Point Iterative Model of a Communication Network . . 137 
7.1 Rank Correlation Among Input Parameters . . . . . . . . . . . . 142

7.2 Illustrative Examples . . . . . . . . . . . . . . . . . . . . . 145

7.2.1 Availability Model of IBM SIP Server Cluster System . . . . . 146

7.2.2 Reliability of a Composite Web Service . . . . . . . . . . . 149

8 Aleatory Model with Simulative Solution 154

8.1 Non-Intrusive Uncertainty Propagation . . . . . . . . . . . . 158

8.1 .1 Illustrative Example . . . . . . . . . . . . . . . 158

8.2 Intrusive Uncertainty Propagation . . . . . . . . . . . . . . . 161

9 Conclusion and Future Work $\quad 164$

A Mathematica, SHARPE and SPNP Input Files 169

A.1 Random Sampling Based Uncertainty Propagation (Chapter 5) . . . 169

A.2 LHS Based Uncertainty Propagation (Chapter 6) $\ldots \ldots \ldots$

A.3 SHARPE Input File for Section $6.2 .2 \ldots \ldots \ldots \ldots$

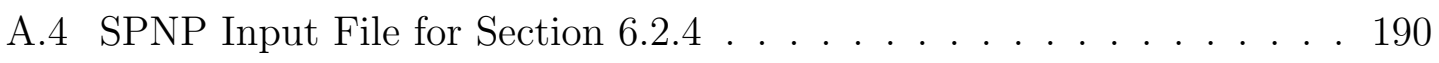

A.5 Mathematica Input File for Section $7 \ldots \ldots \ldots$

A.6 Mathematica Input File for Section 8.1.1 . . . . . . . . . . . 211

A.7 Mathematica Input File for Section $8.2 \ldots \ldots \ldots$

$\begin{array}{ll}\text { Bibliography } & 237\end{array}$

$\begin{array}{ll}\text { Biography } & 245\end{array}$ 


\section{List of Tables}

1.1 Epistemic Uncertainty Propagation for different Aleatory Model types 12

2.1 Epistemic Dependence Vs. Dependence in Aleatory Model . . . . . . 32

5.1 Parameters of the Posterior Distributions . . . . . . . . . . . . . . 95

5.2 Confidence Interval of Blade Center Availability . . . . . . . . . . . . 98

5.3 Conf. Int. using Uniform Epistemic Distributions . . . . . . . . . . 98

5.4 Point Estimates of Reliability Model Parameters . . . . . . . . . . . . 109

5.5 Epistemic Distributions of Parameters . . . . . . . . . . . . 110

6.1 Variance of Sample Mean . . . . . . . . . . . . . . . . . 132 


\section{List of Figures}

3.1 CDF of Reliability of a single-component system, for $\mathrm{r}=10 \ldots 38$

3.2 CDF of reliability of a single-component system at $t=5000$ hours $\ldots 39$

3.3 Expected Reliability of a single-component system at t=1000 hours . 40

3.4 Variance of Reliability of a single-component system at $t=1000$ hours 41

3.5 CDF of reliability of a TMR system at $\mathrm{t}=5000$ hours. . . . . . . . 45

3.6 Expected Reliability of a TMR system at $t=1000$ hours . . . . . . 46

3.7 Variance of Reliability of a TMR system at $t=1000$ hours . . . . . . . 47

3.8 Expected Reliability: Duplex System with one Spare . . . . . . . . . 53

3.9 Variance of Reliability: Duplex System with one Spare . . . . . . . . 55

3.10 Reliability Model of a Recovery Block Architecture . . . . . . . . . . 56

3.11 BPEL representation of Travel-Agent Process . . . . . . . . . . . . . 60

3.12 CTMC Model of Composite Web Service . . . . . . . . . . . . . . . 61

3.13 CDF of Interval Availability by CDD Method . . . . . . . . . . . . 67

3.14 Expected Interval Availability . . . . . . . . . . . . . . . . 68

4.1 Expected Reliability of Composite Web Service . . . . . . . . . . . 73

4.2 CTMC model of M/M/1 system with server breakdown and repair . . 74

4.3 Expected value of Expected Number of Customers in the system . . . 75

4.4 Variance of Expected Number of Customers in the system . . . . . 76

4.5 An ACT for resetting a BGP session . . . . . . . . . . . . . 77

4.6 Expected Probability of Successful Attack, $E\left[P_{\text {goal }}\right] \ldots \ldots$. . . . . . 78 
5.1 Flow Chart of Uncertainty Propagation Method . . . . . . . . . . . . 82

5.2 Top level fault tree of BladeCenter availability Model . . . . . . . . . 93

5.3 CDF of Blade Center Availability : No Standby Blade Server . . . . . 97

5.4 IBM SIP SLEE Cluster (adapted from [85]) . . . . . . . . . . . . . . 99

5.5 Hierarchical Model of the IBM SIP Server Cluster System . . . . . . 100

5.6 CDF of SIP server cluster availability . . . . . . . . . . . . . 102

5.7 REE System Architecture (adapted from [13]) . . . . . . . . . . . . 104

5.8 Flowcharts (adapted from [13]) . . . . . . . . . . . . 105

5.9 Hierarchical Reliability Model of the REE System . . . . . . . . . . . 107

5.10 Markov model for Reliability of Software . . . . . . . . . . . . . 108

5.11 CDF of Reliability of REE System at $t=5$ years $\ldots . . . . .111$

5.12 BPEL representation of Travel-Agent Process . . . . . . . . . . . . . 112

5.13 CTMC Model of Composite Web Service . . . . . . . . . . . . . 113

5.14 CDF of Mean Service Response Time (Random Sampling) . . . . . . 116

5.15 CDF of Reliability of Composite Web Service . . . . . . . . . . 117

6.1 CDF of SIP Server Cluster Availability . . . . . . . . . . . 123

6.2 CDF of Sample Mean of SIP Server Cluster Availability . . . . . . . . 124

6.3 CDF of Reliability of REE System at $t=5$ years $\ldots \ldots . . . .126$

6.4 CDF of Mean of Reliability: LHS Vs. Random Sampling . . . . . . . 128

6.5 CDF of Mean Service Response Time . . . . . . . . . . . . . . 130

6.6 CDF of Reliability of Composite Web Service . . . . . . . . . . . . 132

6.7 CMTS Cluster System Architecture (adapted from [43]) . . . . . . . . 134

6.8 Optional caption for list of figures . . . . . . . . . . . . . . 135

6.9 CDF of COA of the CMTS Cluster System without Rejuvenation . . 137

6.10 CDF of handoff call arrival rate . . . . . . . . . . . . . . 140 
7.1 CDF of SIP server cluster availability . . . . . . . . . . . . . 150

7.2 CDF of Reliability considering independent and correlated parameters 153

8.1 Availability Model: 2 Component System with Shared Repair . . . . 159

8.2 CDF: Availability of 2 Component System with Shared Repair . . . . 160

8.3 CDF of Availability by Intrusive Method . . . . . . . . . . . . . . . 162 


\section{Acknowledgements}

I would like to express my gratitude to my advisor Prof. Kishor S. Trivedi for his constant guidance, encouragement and endless patience during my PhD studies. Without his support, cooperation and understanding, it would not have been possible for me to make any significant progress towards my dissertation. Not only did he guide me with his knowledge and insight, but he himself spent countless hours reviewing, correcting and suggesting improvements to my work. His work ethic, positive attitude and attention to details are a source of inspiration to me. I am grateful to him for his guidance and providing me the opportunity to be his student.

I would like to thank my committee members Prof. John Board, Prof. Krishnendu Chakrabarty, Prof. Henri Gavin and Prof. Benjamin Lee for their kindness to be on my dissertation committee. I thank them for taking the time to read my dissertation and provide suggestions for improvement.

I am indebted to my colleagues at Duke University: I-Yun Liu, Xiaoyan Yin, Jaeshik Lim, Rahul Ghosh, Arpan Roy, Dong-Seong Kim, Fumio Machida and Ermeson Andrade for their support and many useful discussions over the course of my PhD studies. I am grateful to my colleague at Duke University, Amita Devaraj, for her valuable inputs to my research work. I am also grateful to my friend Dazhi Wang, for numerous discussions and spending several hours helping me think through problems. I thank the graduate program coordinator Samantha Morton for helping me through each step of the process. 
I am grateful to my parents and my elder brothers and sisters for their constant encouragement and always putting my needs ahead of their own. I want to thank my wife Radhika Pallavi Mishra and my son Sanatan Mishra for being so understanding and supportive. Without Radhika's support and taking up of even my part of family responsibilities and duties, I would not have been able to complete my dissertation.

I would like to thank my supervisors Chung Yen Chang and Gautam Sharma at NetApp Inc., for their support, understanding and encouragement. Without their cooperation and encouragement, I would not have been able to manage time to perform my research work. I would also like to thank my employer NetApp Inc., for financial support during part of my PhD studies. 


\section{Introduction}

A number of fault tolerance techniques are employed to ensure high dependability of systems in critical applications. Concepts of hardware and software redundancy, automatic fault detection, multiple (escalated) levels of recovery, preventive maintenance, non-disruptive failover etc. are employed to handle both transient and permanent failures $[82,85,43,79,36,46]$. Analytic models are often constructed to assess the dependability of these systems, analyze the effectiveness of the fault tolerance techniques being used and evaluate what-if scenarios.

These stochastic models capture the natural randomness in the system. Randomness in events of interest like times to failure/recovery of components, ability to detect failures, ability to perform recovery action, time to arrival, service time, etc. are taken into account by means of their distributions. This inherent randomness in the system or its environment, taken into account by stochastic models, is called aleatory uncertainty (also known as variability, stochastic uncertainty or irreducible uncertainty). These stochastic or aleatory models are mostly solved assuming fixed values for the model input parameters.

In real life, the parameters of the aleatory model may be determined from lifetime 
experiments, field failure data, maintenance logs or other sources of observed data. These data sources will provide a finite number of observations, leading to uncertainty in the estimates of model input parameters computed using the observed data. In many cases, the values of parameters may not be even based on observed data, but on expert guesses or theoretical arguments (other sources of uncertainty). This uncertainty due to lack of complete information about the parameter values, is known as epistemic uncertainty (also known as reducible uncertainty) [83, 59].

The input parameters of analytic models have been considered as unknowable and hence the computation of availability or reliability by solving models at specific parameter values, not very useful, by Singpurwalla [80]. Due to the epistemic uncertainties, the availability or reliability computed using point estimates of parameter values obtained from measurements is considered to be conditional upon the values of the parameters. Singpurwalla coins the term survivability to denote the unconditional value of reliability after the uncertainty in parameter values are taken into account.

Parametric or epistemic uncertainty introduces uncertainty in the model output. To compute the uncertainty in model output value, the epistemic uncertainties in each of the model input parameters need to be propagated through the stochastic model. The uncertainty in the model output due to the epistemic uncertainty in the input parameters, can then be summarized in the form of distribution, variance or confidence intervals of the model output values.

\subsection{Epistemic Uncertainty Propagation}

Methods of epistemic uncertainty propagation can be broadly classified into intrusive and non-intrusive methods. Intrusive methods manipulate the aleatory model to perform the uncertainty propagation. While intrusive epistemic uncertainty propagation can be easily performed through aleatory models with simulative solutions, 
they are harder to perform through analytic aleatory models. Perturbation methods, which formulate and solve a version of the original model with the model parameters incorporating the epistemic uncertainty, are examples of intrusive methods of epistemic uncertainty propagation through analytic aleatory models [65]. On the other hand, non-intrusive methods use repeated solutions of the existing model with different parameter values. In case of an aleatory model with simulative solution, uncertainty propagation can be performed in both intrusive and non-intrusive way. In the non-intrusive method, epistemic uncertainty propagation becomes a two-phase simulation, where the first phase takes into account the epistemic uncertainty while the second phase accounts for aleatory uncertainty $[66,57,91]$. The intrusive method involves only a single phase simulation where both epistemic and aleatory uncertainty is considered together. During the execution of the simulation model, each realization from the aleatory distribution accounts for both the aleatory as well as epistemic uncertainty. The single distribution of model output thus obtained upon performing the simulation incorporates both the epistemic as well as the aleatory uncertainty $[57]$.

Intrusive methods of epistemic uncertainty propagation seem to be suitable only for small models with a small number of input parameters. For most real-life systems with more complex models and several input parameters, they are hard to implement and are computationally intensive. Non-intrusive methods of uncertainty propagation rely on multiple solutions of the already existing model. They do not modify or perform any manipulation to the model or the model output and use the existing model as such. While intrusive methods are difficult to implement for complex analytic aleatory models, intrusive uncertainty propagation through aleatory models with simulative solutions can be performed with an effort comparable to that needed for non-intrusive uncertainty propagation. Several non-intrusive analytic methods, which perform manipulations on the aleatory model output, exist. 
Analytic methods for parametric epistemic uncertainty propagation, in terms of computing the confidence interval for system reliability of simple systems have been researched. Madansky et. al. [47] estimate the approximate confidence interval for reliability of series and parallel systems, when the reliability of individual components as well as the number of successes and total number of trials used in estimating the reliability of each of the components is known. The method assumes independent failures of components and the reliability of the components to be the parameters of Bernoulli aleatory distribution. It makes use of the result that a multiple of the natural logarithm of the likelihood ratio test statistic of reliability follows a chisquare distribution with 1 degree of freedom. Again assuming independent failures of components and the reliability of components to be parameter of Bernoulli aleatory distribution, Easterling et. al. [19] compute the approximate confidence interval of reliability of series and parallel systems using the reliability test data of the components. This method treats the overall reliability of the system to be a parameter of a Bernoulli aleatory distribution with the parameter of this distribution obtained by matching the variance of the reliability computed by the maximum likelihood estimation (MLE) method to that computed under the Bernoulli assumption. It then uses the incomplete beta function to estimate the confidence interval for reliability. Sarkar [74] and Lieberman et. al. [42] provide methods to calculate the exact confidence interval (lower bound) of reliability of a series system, with time to failure of each component following an exponential distribution (aleatory). This method makes use of the chi-square statistic to compute the confidence interval of the overall system reliability. To understand the method explained by Sarkar in [74], assume $X_{1}{ }^{(i)}<X_{2}{ }^{(i)}<\ldots<X_{r}{ }^{(i)}$ be the order statistics of $r$ observed values of times to failure of $i^{\text {th }}$ component in a series system with $k$ components. The time to failure of $i^{\text {th }}$ component is assumed to follow an exponential distribution with parameter $\lambda_{i}$, 
$i=1,2, \ldots, k$. Consider the following expressions:

$$
\begin{aligned}
D_{1}{ }^{(i)} & =X_{1}{ }^{(i)}, \\
D_{j}{ }^{(i)} & =X_{j}^{(i)}-X_{j-1}{ }^{(i)}, j=2, \ldots, r, \\
\Delta_{j}{ }^{(i)} & =(r-j+1) D_{j}{ }^{(i)}, j=2, \ldots, r, \\
Z_{j} & =\min _{i=1, \ldots, k} \Delta_{j}{ }^{(i)}, j=2, \ldots, r \\
V & =\sum_{j=1}^{r} Z_{j}
\end{aligned}
$$

Making use of the result that $2 r V\left(\lambda_{1}+\lambda_{2}+\ldots+\lambda_{k}\right)$ follows a chi-square distribution with $2 r$ degrees of freedom, the lower limit of $100(1-\alpha) \%$ confidence interval of reliability of series system is given in [74] by $e^{-t \chi^{2}{ }_{2 r}(\alpha) / 2 V}$. where, $\chi_{2 r}{ }^{2}(\alpha)$ is a critical value from chi-square distribution with $2 r$ degrees of freedom.

Mawaziny and Buehler [49] provide a large-sample approximation to the confidence interval for the reliability of a series system when the times to failure of the components are exponentially distributed (aleatory).

Most of the above methods are very limited in their range of application. These methods can be applied only to simple series-parallel systems. They rely on the fact that the model output is a closed-form expression of the input variables and and hence the output distribution can be derived from input distributions. However, deriving the confidence interval or distribution of the model output becomes intractable for larger number of input parameters or models more complex than simple series or series-parallel systems.

Analytic methods of Coit [16] provide a way of estimating the confidence interval of reliability for more complex non-state-space models exploiting the linearity property of expectation, linear transformations of variance and independence of failures of the components. This method works for any series-parallel system and hence is 
more widely applicable than the methods discussed so far. It takes the variance of the reliability of individual components, as its input, to estimate variance of overall system reliability. If $R_{i}$ is the reliability of the $i^{t h}$ component of a series system with $N$ components, assuming component reliabilities to be statistically independent, the reliability of the system is given by:

$$
R_{\text {series }}=R_{1} \cdot R_{2} \ldots R_{N}
$$

The variance of $R_{\text {series }}$ can be computed as:

$$
\begin{aligned}
\operatorname{Var}\left[R_{\text {series }}\right] & =\operatorname{Var}\left[R_{1} \cdot R_{2} \ldots R_{N}\right] \\
& =E\left[\left(R_{1} \cdot R_{2} \ldots R_{N}\right)^{2}\right]-\left(E\left[R_{1} \cdot R_{2} \ldots R_{N}\right]\right)^{2} \\
& =E\left[R_{1}^{2}\right] \cdot E\left[R_{2}^{2}\right] \ldots E\left[R_{N}^{2}\right]-\left(E\left[R_{1}\right] \cdot E\left[R_{2}\right] \ldots E\left[R_{N}\right]\right)^{2} \\
& =E\left[R_{1}^{2}\right] \cdot E\left[R_{2}^{2}\right] \ldots E\left[R_{N}^{2}\right]-\mu_{1}^{2} \cdot \mu_{2}^{2} \ldots \mu_{N}{ }^{2} \\
& =\left(\prod_{i=1}^{N}\left({\sigma_{i}}^{2}+\mu_{i}^{2}\right)\right)-\mu_{1}^{2} \cdot \mu_{2}^{2} \ldots \mu_{N}^{2}
\end{aligned}
$$

where, $\mu_{i}$ and $\sigma_{i}^{2}$ are the estimates of mean and standard deviation of reliability of $i^{\text {th }}$ component of the system, computed from observed lifetime data.

The reliability of a parallel system with $N$ components is given by:

$$
R_{\text {parallel }}=1-\left(1-R_{1}\right)\left(1-R_{2}\right) \ldots\left(1-R_{N}\right)
$$

The variance of parallel system reliability is given by:

$$
\begin{aligned}
\operatorname{Var}\left[R_{\text {parallel }}\right] & =\operatorname{Var}\left[1-\left(1-R_{1}\right)\left(1-R_{2}\right) \ldots\left(1-R_{N}\right)\right] \\
& =\operatorname{Var}\left[\left(1-R_{1}\right)\left(1-R_{2}\right) \ldots\left(1-R_{N}\right)\right]
\end{aligned}
$$

Substituting $Y_{i}=1-R_{i}, \operatorname{Var}\left[\left(1-R_{1}\right)\left(1-R_{2}\right) \ldots\left(1-R_{N}\right)\right]$ reduces to $\operatorname{Var}\left[Y_{1} . Y_{2} \ldots Y_{N}\right]$ which is of the same form as the expression for variance of reliability of a series system and performing similar manipulations, the variance can be 
obtained as:

$$
\begin{aligned}
\operatorname{Var}\left[R_{\text {parallel }}\right] & =\operatorname{Var}\left[Y_{1} \cdot Y_{2} \ldots Y_{N}\right] \\
& =E\left[\left(Y_{1} \cdot Y_{2} \ldots Y_{N}\right)^{2}\right]-\left(E\left[Y_{1} \cdot Y_{2} \ldots Y_{N}\right]\right)^{2} \\
& =E\left[Y_{1}^{2}\right] \cdot E\left[Y_{2}^{2}\right] \ldots E\left[Y_{N}^{2}\right]-\left(E\left[Y_{1}\right] \cdot E\left[Y_{2}\right] \ldots E\left[Y_{N}\right]\right)^{2} \\
& =E\left[Y_{1}^{2}\right] \cdot E\left[Y_{2}^{2}\right] \ldots E\left[Y_{N}^{2}\right]-\mu_{1}^{2} \cdot \mu_{2}^{2} \ldots \mu_{N}^{2} \\
& =\left(\prod_{i=1}^{N}\left(\sigma_{i}{ }^{2}+\left(1-\mu_{i}\right)^{2}\right)\right)-\prod_{i=1}^{N}\left(1-\mu_{i}\right)^{2}
\end{aligned}
$$

The method explained by Coit in [16] partitions the system into blocks where each block consists of components either entirely in series or entirely in parallel. Then it uses the variances computed using Equations 1.3 and 1.6 to compute the overall variance. It also computes the lower limit of confidence interval of system reliability, assuming the system reliability to follow a lognormal distribution with parameters computed using the mean system reliability and variance of system reliability (as computed using Equations 1.3 and 1.6). This method is applicable only to nonstate-space models. Among non-state-space models, it can be applied only to those which have a series-parallel structure (and is not applicable to bridge configurations)

Another method that uses the moments of the model output and the input parameters has been discussed by Yin et. al. in [93]. It first gets a Taylor series expansion of the model output, discards higher order terms, makes use of the properties of variance and expectation and then computes the variance of overall reliability. Assume that the expression for reliability of a system, given by $R(t)$, is a function of $m$ parameters $\left\{\lambda_{i}, i=1, \ldots, m\right\}$. The reliability $R(t)$ can be written as a second order Taylor series (ignoring higher order terms):

$$
\begin{array}{r}
\left.R(t) \approx R(t)\right|_{\mu}+\left.\sum_{i=1}^{m}\left(\lambda_{i}-\mu_{i}\right) \frac{\partial R(t)}{\partial \lambda_{i}}\right|_{\mu}+\sum_{i=1}^{m} \frac{\left(\lambda_{i}-\mu_{i}\right)^{2}}{2} \\
\left.\quad \frac{\partial^{2} R(t)}{\partial \lambda_{i}^{2}}\right|_{\mu}+\left.\sum_{i=1}^{m} \sum_{j=1}^{i-1}\left(\lambda_{i}-\mu_{i}\right)\left(\lambda_{j}-\mu_{j}\right) \frac{\partial^{2} R(t)}{\partial \lambda_{i} \partial \lambda_{j}}\right|_{\mu} .
\end{array}
$$


where, $\left.R(t)\right|_{\mu}$ is the reliability of the system evaluated at the mean of the input parameters of the reliability model $\left(\mu=\mu_{1}, \mu_{2}, \ldots, \mu_{m}\right)$, where $\mu_{i}$ is the mean of the parameter $\lambda_{i}$ ). Similarly, $\left.\frac{\partial R(t)}{\partial \lambda_{i}}\right|_{\mu},\left.\frac{\partial^{2} R(t)}{\partial \lambda_{i}^{2}}\right|_{\mu}$ and $\left.\frac{\partial^{2} R(t)}{\partial \lambda_{i} \partial \lambda_{j}}\right|_{\mu}$ are the partial derivatives of reliability, evaluated at $\mu$. The expected reliability, $E[R(t)]$, is given by:

$$
\left.E[R(t)] \approx R(t)\right|_{\mu}+\left.\sum_{i=1}^{m} \frac{\sigma_{i}^{2}}{2} \frac{\partial^{2} R(t)}{\partial \lambda_{i}^{2}}\right|_{\mu}
$$

and the variance, $\operatorname{Var}[R(t)]$, is given by

$$
\begin{aligned}
\operatorname{Var}[ & R(t)]\left.\approx \sum_{i=1}^{m} \sigma_{i}^{2}\left(\frac{\partial R(t)}{\partial \lambda_{i}}\right)^{2}\right|_{\mu}+\left.\frac{1}{2} \sum_{i=1}^{m} \sigma_{i}^{4}\left(\frac{\partial^{2} R(t)}{\partial \lambda_{i}^{2}}\right)^{2}\right|_{\mu} \\
& +\left.\sum_{i=1}^{m} \sum_{j=1}^{i-1} \sigma_{i}^{2} \sigma_{j}^{2}\left(\frac{\partial^{2} R(t)}{\partial \lambda_{i} \partial \lambda_{j}}\right)^{2}\right|_{\mu}
\end{aligned}
$$

Obtaining the derivatives of the expression for model output is computationally intensive and so the method discussed in [93] can be applied only to simple models.

In case the uncertainty in input parameters is given as deterministic bounds rather than confidence intervals, computing bounds on the model output due to the perturbations in the input parameters is appropriate. Ramesh et. al. derive analytic bounds for model output for variations (perturbations) in value of a single parameter or values of multiple parameters [65] for Markov reliability models, with the help of differential equations based on the transient solution of state probabilities (obtained by solving the Kolmogorov differential equations) [86].

Parametric sensitivity analysis via derivatives of the output measure with respect to input parameters has also been used frequently in this context. Sensitivity analysis helps identify how the variability in the model output is connected to any of the model input parameters and hence rank input parameters in order of their contribution to model output $[75,93,5]$. 
Some other methods of propagation of epistemic uncertainty (although discussed in the context of deterministic models and not stochastic models), like response surface methodology (RSM), Fourier amplitude sensitivity test (FAST) and fast probability integration (FPI) have been reviewed in [30]. The FPI and FAST methods are computationally complex (more so with larger number of input parameters) and require the model output to be closed-form expressions of the inputs.

In the RSM method, based on experimental design, values from input parameters are selected depending on their range (say a low, mid and high value) and the model is evaluated at the set of chosen parameter values. A response surface is constructed out of the model output values, thus obtained. This response surface maybe constructed by fitting a regression model to the set of output values obtained. Uncertainty propagation is then carried out on this response surface (using any of the methods like Taylor series expansion or sampling based method), in place of the original model. This method is also called method of reduced order model or surrogates, as it is based on a simplification of the original mathematical problem. The RSM method requires knowledge of experimental design to select the inputs. The uncertainty analysis in RSM method is not carried out directly on the model but on a response surface approximation to the model, which itself can be difficult to construct with high fidelity.

Polynomial Chaos Expansion method is an analytical method for non-intrusive propagation of epistemic uncertainty. It entails obtaining an expansion of the model output based on a suitable choice of orthogonal polynomial and its associated stochastic process to represent the epistemic uncertainty in input parameters. The variance and expectation of the output metric is computed from the coefficients of the expansion. The method involves computing the coefficients of the expansion. Obtaining the coefficients of expansion accurately is computationally expensive, more so when the number of parameters is large. Since the expansions result in infinite series, 
they need to be truncated at some suitable order, which introduces errors. Therefore this method not only has computational complexity with larger models or more parameters, accuracy of this method is also an issue [17].

Sampling based uncertainty propagation is a popular method of epistemic uncertainty propagation because of its wide range of applicability and feasibility even with large number of input parameters or large complex models, even though it may be computationally more intensive than analytic methods for simpler models with fewer parameters where uncertainty propagation via analytic methods may be possible [30, 28]. It also enables consideration of correlation between input parameters more easily than the analytical methods [30, 35].

Haverkort et. al. [28] compute the mean and variance of the model outputs by the method of parametric sensitivity analysis as well as the quantiles of the distribution of model output (along with mean and variance) by propagating the uncertainty through the model using Monte Carlo sampling. They also consider some degree of dependence between the parameters.

\subsection{Contribution of this Dissertation}

In this dissertation we address the computation of uncertainty in output metrics of stochastic models due to epistemic uncertainty in model input parameters, with a focus on dependability and performance models of current computer and communication systems.

Due to epistemic uncertainties, the output of dependability or performance models computed using fixed parameter values (e.g., point estimates of parameter values obtained from measurements) can be considered to be conditional upon the values of the input parameters used. By unconditioning the model output with respect to the model input parameter values (considering the epistemic uncertainty), the uncertainty in the model output metric can be estimated. This unconditioning can be 
done by means of a multi-dimensional integration.

Depending on the aleatory model type and complexity of its output, different methods need to be applied to perform this unconditioning integration. Simple analytic aleatory models may be solved analytically to get the model output as closedform expressions of model input parameters. For example, in case of a simple tripe modular redundancy (TMR) system, assuming all components of the system follow independent and identically distributed exponential failure law, with parameter $\lambda$, the reliability model has one input parameter $\lambda$ and the model output metric (reliability) can be expressed as $R(t)=3 e^{-2 \lambda t}-2 e^{-3 \lambda t}$, which is a closed-form expression of $R(t)$, in terms of the model input parameter $\lambda[86]$. On the other hand, for complex models such as the availability model of an IBM SIP SLEE server, as shown in [85], analytic closed-form expressions of output metrics are not possible and the model can only be solved analytic-numerically using tools like SHARPE [87] or SPNP [32]. Alternatively, the aleatory model may be solved via a simulation. Different methods of propagating epistemic uncertainty need to be employed for each of these cases. For analytic models which have simple closed-form model outputs, unconditioning by direct analytic integration may be possible. For more complex analytic models with closed form solutions, the expression for model output may not be amenable to analytic integration (complex expression and/or too many parameters) and numerical integration maybe needed. Sampling based uncertainty propagation $[18,55]$ can be applied to complex analytic models (can be used when the model can be solved either analytically or analytic-numerically), as well as models with simulative solutions. Table 1.1 summarizes the applicability of various methods of epistemic uncertainty propagation, for different types of aleatory models.

In this dissertation, we present an approach for propagation of parametric epistemic uncertainty through stochastic aleatory models of varying complexity. Uncertainty propagation is carried through a wide range of model types (ranging from 
Table 1.1: Epistemic Uncertainty Propagation for different Aleatory Model types

\begin{tabular}{|l|l|l|l|}
\hline & \multicolumn{3}{|l|}{ Epistemic Uncertainty Propagation Method } \\
\hline & $\begin{array}{l}\text { Analytic } \\
\text { Closed- Inte- } \\
\text { Form } \\
\text { gration }\end{array}$ & $\begin{array}{l}\text { Numerical } \\
\text { Integration }\end{array}$ & $\begin{array}{l}\text { Sampling } \\
\text { Based }\end{array}$ \\
\hline $\begin{array}{l}\text { Analytic } \\
\text { Model } \\
\text { Closed-Form } \\
\text { solution }\end{array}$ & $\begin{array}{l}\text { Applicable } \\
\text { (simple ex- } \\
\text { pressions) }\end{array}$ & Applicable & Applicable \\
\hline $\begin{array}{l}\text { Analytic } \\
\text { Model } \\
\text { Numerical } \\
\text { Solution }\end{array}$ & $:$ & $\begin{array}{l}\text { Not Applica- } \\
\text { ble Applicable }\end{array}$ & Applicable \\
\hline $\begin{array}{l}\text { Analytic } \\
\text { Model } \\
\text { Simulative } \\
\text { Solution }\end{array}$ & $:$ & $\begin{array}{l}\text { Not Applica- } \\
\text { ble Applicable }\end{array}$ & Applicable \\
\hline
\end{tabular}

simple models with closed-form expression of model output to large complex hierarchical models where only analytic-numeric solution is possible and even models with simulative solution), with different model output metrics. For simple aleatory models (with closed form expression of model output) the expressions for expectation, variance and distribution of model output metric due to the epistemic uncertainties, are derived and analyzed to understand their limiting behavior.

The contributions of this dissertation can be summarized as:

- An approach for epistemic uncertainty propagation is developed and applied to a wide range of stochastic dependability and performance models for computer systems. These models range from simple non-state-space models with a few input parameters to large state space models or even hierarchical models with more than fifty input parameters. Some of the model output metrics considered are reliability and availability of computer systems, response time of a web service, capacity oriented availability of a communication system, security (probability of successful attack) of a network routing session, expected number of jobs in a queueing system with breakdown and repair of servers and call 
handoff probability of a cellular wireless communication cell.

- For analytic models where the model output can be obtained as simple closed form expression of model input parameters, exact expressions for distribution, expectation and variance of the model output are derived and analyzed to gain insight into the uncertainty in model output, due to the epistemic uncertainty in model input parameters. Limiting behavior of metrics of uncertainty in model output, is also studied. Expressions for distribution, expectation and variance of the model output are derived for reliability models of several simple non-repairable systems and also for availability of a simple systems.

- A sampling based non-intrusive approach for epistemic uncertainty propagation through large and complex aleatory models is presented. This method is more widely applicable than existing analytic methods (as discussed in Section 1.1). This method can be applied when observed lifetime data about system and its components is directly available to the modeler, as well as when only processed information about epistemic uncertainty is available, in the form of confidence intervals of the input parameters. The form and parameters of the epistemic distributions are not arbitrarily assumed (as is done in other sampling based methods as discussed in Section 1.1, for example [28]), but derived based on the aleatory distribution and the provided confidence intervals. The method of obtaining the form and parameters of epistemic distribution is also presented. The sampling method used in this approach is more robust (demonstrated in the illustrative examples considered) and more likely to cover the entire distribution range of the input parameter. The confidence interval of reliability (and other model output metrics) is obtained using a non-parametric method, obviating any need for assuming or fitting any distribution to the model outputs. 
- The sampling based epistemic uncertainty method is successfully applied for uncertainty propagation through several dependability and performance models of computer systems, including rather large hierarchical models with a large number of model input parameters.

- The sampling based method, also enables considering correlation in the model input parameter values (epistemic dependence), even without sampling from the joint distribution function of correlated parameters. Uncertainty propagation considering epistemic dependence between input parameters is also applied to some of the dependability and performance models considered.

- The entire uncertainty propagation approach is shown to be independent of model solution tool or technique as it is applied to non-state-space, state-space, hierarchical, simulative and fixed-point iterative models solved using SHARPE, SPNP and Mathematica software packages $[87,32,2]$

\subsection{Outline of the Dissertation}

This dissertation is organized as follows:

In Chapter 2, we provide the background on analytic aleatory models used for dependability and performance analysis of computing and communication systems. We also introduce some basic concepts and derive results which are used throughout the dissertation.

In Chapter 3, we present the closed-form approach for epistemic uncertainty propagation through analytic aleatory models. We explain the method in detail, derive closed-form expressions for distribution, mean and variance of model output, due epistemic uncertainty and analyze the results to understand their limiting behavior. We illustrate the method with the help of several examples and analyze the results.

In Chapter 4, we present a numerical integration based approach for epistemic 
uncertainty propagation through analytic aleatory models. As the analytic models become more complex, closed-form approach for epistemic uncertainty propagation may no longer be possible and in such cases, a numerical integration based approach may be needed. We explain this approach and illustrate it with the help of several examples.

In Chapter 5, a sampling based epistemic uncertainty propagation method which can be applied to a wide range of analytic aleatory model types, from simple nonstate space models to complex hierarchical models is presented. This method acts as a wrapper to existing models and their solution types. The method is illustrated with the help of several real-world examples.

In Chapter 6, we improve upon the sampling based epistemic uncertainty propagation explained in Chapter 5 by using the latin hypercube sampling method in place of random sampling. We illustrate this uncertainty propagation method with several real-world examples and demonstrate that this method provides more robust estimates as compared to the one presented in Chapter 5.

In Chapter 7, we relax the assumption of epistemic independence between the input parameters of the aleatory model, considered in Chapters 3 through 6 and present a method to incorporate correlations between the aleatory model input parameters as random variables into the sampling based uncertainty propagation method explained in Chapter 6. We illustrate the sampling based method for epistemic uncertainty propagation in case of correlated input parameters, with the help of examples.

In Chapter 8, the sampling based uncertainty propagation method is applied to an aleatory model with simulative solution. We illustrate this with the help of a simple simulation model and discuss ways of interpreting the two levels of uncertainty in model output due to epistemic as well as aleatory uncertainty.

In Chapter 9, we provide the summary of this dissertation. 


\section{2}

\section{Background}

In this dissertation, we perform uncertainty propagation through dependability and performance models of current computer and communication systems. Therefore, in this chapter, we first provide a brief overview of the analytic modeling techniques used by the dependability and performance models in this dissertation. We then introduce some basic concepts and derive results that are used throughout the dissertation. We perform derivations for the epistemic distributions for parameters of exponential and Bernoulli aleatory distribution and explain the difference between aleatory and epistemic dependence.

\subsection{Analytic Aleatory Model Types}

Depending on the level of detailed system behavior to be represented by the aleatory model, the output measures and dependencies between events in the system (aleatory

dependence), state space, non-state space or hierarchical modeling techniques can be applied. 


\subsubsection{Non State Space Models}

Non state-space models represent the system operational state (UP or DOWN) based on the operational state of individual components in the system. They rely on the basic assumption of statistical independence of each component's failure and repair. They are most often used for reliability analysis of non-repairable systems. They can also be used for availability analysis for repairable systems, in which each component in the system has statistically independent failure and repair. The operational state of a system, modeled by a non state space model, can be represented with help of a structure function which is defined as follows [86]:

Definition Let $\boldsymbol{X}=x_{1}, x_{2}, \ldots, x_{n}$ be a state vector of the system with $n$ components, such that

$$
x_{i}= \begin{cases}1 & \text { if component } i \text { is up } \\ 0 & \text { if component } i \text { is down }\end{cases}
$$

The structure function $\phi(\boldsymbol{X})$ is defined by:

$$
\phi(\boldsymbol{X})= \begin{cases}1 & \text { if system is up } \\ 0 & \text { if system is down }\end{cases}
$$

In the remainder of this section we provide a brief overview of some of the non state space analytic model types.

\section{Reliability Block Diagram}

In a reliability block diagram, each component of the system is represented as a block and these blocks are then connected in series/parallel or $k-$ out $-o f-n$ combinations based on operational dependencies.

For a system that needs each of its components to be operational, the blocks need to be connected in series. The structure function of an $n$-component series system is 
given by:

$$
\phi_{\text {series }}(\boldsymbol{X})=x_{1} \cap x_{2} \cap \ldots \cap x_{n}
$$

When the system is operational if at least one of the components is operational, the blocks are connected in parallel. The structure function of an $n$-component parallel system is given by:

$$
\phi_{\text {parallel }}(\boldsymbol{X})=x_{1} \cup x_{2} \cup \ldots \cup x_{n}
$$

If the system is operational as long as at least $k$ out of the total $n$ components in the system are operational, the blocks are connected in a $k$-out-of-n combination. The structure function of an $k-$ out $-o f-n$ system is given by:

$$
\phi_{k o f n}(\boldsymbol{X})=\cup_{|J|=k}\left(\cap_{j \in J} x_{j}\right)
$$

A system can be modeled with components in combinations of series, parallel or $k-$ out - of $-n$ configurations.

Fault Trees

While the reliability block diagrams represent the conditions under which a system will be operational, fault trees represent conditions under which a system will fail. A fault tree is a representation of the combination of events that can cause a system failure. The root or top-level of the tree represents system failure. An event at any level is reduced to a combination of lower- level events by means of logic gates until a basic event is reached. The types of gates are and gate, or gate, not gate and $k-$ out $-o f-n$ gate. Each gate can have more than one input but only one output. Inputs to a gate can be either the primary event or outputs from other gates. The output of each gate is determined by boolean logic (output of an and gate is logic 1 iff all its inputs are logic 1; output of an or gate is a logic 1 if any of the inputs is a logic 1 ; output of a $k-$ out $-o f-n$ gate is a logic 1 if at least $k$ of the $n$ inputs are $\operatorname{logic} 1)$. 
Several algorithms for dependability analysis using non-state-space models exist. The most commonly used algorithms include state enumeration [10], factoring [76], inclusion/exclusion [77], sum of disjoint products (SDP) [64], and binary de-

cision diagrams (BDD) [11]. Compared to other methods, BDD-based algorithms are more powerful and efficient in dependability evaluation because of their concise representation of Boolean functions and the ease of BDD manipulations [89].

\subsubsection{State Space Models}

The non-state-space models cannot easily handle dependencies in failure/repair of components or shared repair facilities. Complex inter-component interaction is also difficult to model using the non-state space models. In such complex cases, state space models are required. State-space models use stochastic process to model the states and state transitions of a system as it progresses in time.

State space models like Markov chains [61, 86], stochastic Petri nets, stochastic reward nets [34], semi-Markov processes [78] and Markov Regenerative Processes $[45,44]$ have been widely used to model not only dependability of complex computer systems, but also for performance and performability analysis of systems [43].

\section{Markov Chains}

A Markov process is the underlying stochastic process of Markov chain models. A Markov process is a stochastic process where the probability distributions for its future development depends only on the current state and not how the process arrived to that state [86]. A Markov process with a discrete state space is known as a Markov chain. If the state change in a Markov chain can happen only at discrete time points, the Markov chain is known as a discrete time Markov chain (DTMC). If the state change can happen at any time, it is called a continuous time Markov chain (CTMC). The models used for uncertainty propagation in this dissertation are CMTCs. 
The discrete space continuous time stochastic process $X(t) \mid t \geq 0$ is called a continuous time Markov chain if:

$P\left(X(t)=x \mid X\left(t_{n}\right)=x_{n}, X\left(t_{n-1}\right)=x_{n-1}, \ldots, X\left(t_{0}\right)=x_{0}\right)=P\left(X(t)=x \mid X\left(t_{n}\right)=x_{n}\right)$

where, $t_{0}<t_{1}<t_{2}<\ldots<t_{n} \ldots<t$ In other words, the probability of being in the state $x$ at time $t$ depends only on the fact that the process is in state $x_{n}$ at time $t_{n}$ and not where it was before that. If Equation 2.1 holds for any start time $t_{n}$ and depends only on time $t-t_{n}$, the CTMC is called a homogeneous Markov chain. It is important to note that the sojourn time in each state, as well as the transition time from one state to another in a CTMC, is exponentially distributed.

The transient behavior of a CTMC is described by the Kolmogorov differential equation as [86]:

$$
\frac{d \boldsymbol{\pi}(t)}{d t}=\boldsymbol{\pi}(t) \boldsymbol{Q}
$$

where, $\boldsymbol{\pi}(t)=\pi_{1}(t), \pi_{2}(t), \ldots, \pi_{n}(t)$ is the state probability vector (probability of being in any of the $n$ states of the CTMC, at time $t$ ) and $\boldsymbol{Q}=\left[q_{i j}\right]_{n \times n}$ is known as the infinitesimal generator matrix with $q_{i j}$ being the rate of transition from state $i$ to state $j$ and $q_{i i}=-\sum_{i \neq j} q_{i j}$. In steady state (i.e. as $t \rightarrow \infty$ ), Equation 2.2 reduces to:

$$
\pi Q=0
$$

where $\boldsymbol{\pi}=\pi_{1}, \pi_{2}, \ldots, \pi_{n}$ is the steady state probability vector.

The state probability vectors for transient or steady state analysis of CTMCs are obtained by solving Equations 2.2 and 2.3, respectively.

For the dependability analysis of systems, the Markov chain is drawn as state transition diagram and the arcs representing transition from one state to another are labeled by the rate corresponding to the exponentially distributed time of the 
transition. Markov chain models can be extended by assigning reward rates to the states to obtain Markov reward models. A reward rate vector $\boldsymbol{r}$ is defined over the states of the CTMC with a reward rate $r_{i}$ assigned to state $i$. By defining specific reward structure and using the measures expected instantaneous reward rate $E I R=\boldsymbol{r}^{\boldsymbol{T}} \boldsymbol{\pi}(\boldsymbol{t})$ and expected accumulated reward rate $E A R=\sum_{i=1}^{n} r_{i} \int_{0}^{t} \pi_{i}(t)$, various measures like reliability, availability, performance and performability can be obtained (e.g. a reward rate of 1 for the "up" states and 0 for the "down" states to obtain reliability or availability) [65]. Solutions to Markov chain models and the reward rate computations can be performed by hand for small Markov chain models for larger Markov chain models software packages like SHARPE [87] can be used.

\section{Stochastic Reward Nets}

When the number of states of a Markov chain becomes prohibitively large, automated generation of the state space is needed. A stochastic Petri net (SPN) can be used to automatically generate the state space. It is a directed graph with two disjoint types of nodes: places and transitions with directed arcs connecting places to transitions. A positive integer called multiplicity can be associated with each arc. Each place may contain zero or more tokens. A transition is enabled when each of its input places has at least as many tokens as the multiplicity of the corresponding input arc. After a transition is enabled, it might fire after a certain amount of time has elapsed which is exponentially distributed with a rate known as its firing rate. Upon firing, a number of tokens equal to the multiplicity of the input arc is removed from each of the input places and a number of tokens equal to the multiplicity of the output arc are deposited at each of its output places. The number of tokens at the places at any instance is known as the marking and it is the state of the system at that instant. Keeping track of the number of tokens in all the places generates the underlying CTMC (the marking process). Thus SPN provides a way of concise representation 
of CTMCs. A stochastic reward net (SRN) is an extension of the SPN with notions of reward functions and several marking dependent features that greatly simplify the graphical representation of the model $[15,86]$.

A large variety of reward-based measures can be calculated with the help of SRN to perform dependability, performance and performability analysis of systems $[48,43]$.

\section{Semi-Markov and Markov Regenerative Process Models}

Markov chain models assume the time to the next state transition is exponentially distributed. Similarly stochastic Petri nets and stochastic reward nets also assume the firing times of transitions to be exponentially distributed. In cases where times to transition or firing times are not exponentially distribution, semi-Markov process (SMP) and Markov regenerative process (MRGP) models may be used. These models are based on the concepts of Markov renewal theory [39]. Markov regenerative stochastic Petri net (MRSPN) [14] is a concise way of representing an MRGP. MRGP and MRSPNs have been used in availability modeling [20, 78, 45, 44, 92]. Solution

techniques for MRGPs and MRSPNs have been discussed in [14, 39, 62]. Solving MRGP and SMP models by hand might often be time consuming. Software packages like SHARPE [87] can be used for obtaining steady state solutions.

\subsubsection{Hierarchical Models}

A hierarchical model consists of multiple levels of models, where the lower-level models capture the detailed behavior of subsystems and the topmost level is the system-level model. Hierarchical availability models can be constructed for cases where failures and repairs in subsystems are independent of each other.

Modeling of large systems in a one composite model might lead to state space explosion even with concise state representation like SRNs or SPNs. Hierarchical 
models scale better with the number of subsystems and subsystem components than does a composite model and thus help avoid largeness in the state space of models [6]. Besides, large difference in magnitudes of parameter values can lead to stiffness in the overall model [33]. A multilevel model helps avoid this problem. In many cases it is also more convenient and intuitive to model parts of the system(subsystems) individually rather than the whole system at once and then have a model on top of these sub-system models to account for the interactions between these lower level models. Using a combinatorial model on the top level reduces the number of states considerably [6]. The ability of tools like SHARPE [87] to combine results from different kinds of models makes it possible to use state-space methods for those parts of a system that require them, and use non-state-space methods for other parts.

Hierarchical models are widely used for dependability analysis of complex computer systems $[85,13,82]$.

\subsection{Bayes' Theorem}

In statistical inference, conclusions are drawn about the characteristics of the population from observed numerical data. Estimates of the parameters of the distribution of the population are computed from observed data. Assume that $X_{1}, X_{2}, \ldots, X_{r}$ are the independent and identically distributed random variables denoting the $r$ observed values from population with distribution parameter $\theta$. The continuous form of Bayes' theorem provides the probability density function of the parameter of distribution (as random variable) $\Theta$, conditioned on the observed data $x_{1}, x_{2}, \ldots, x_{n}$, as:

$$
f_{\Theta \mid X_{1}, X_{2}, \ldots, X_{n}}\left(\theta \mid x_{1}, x_{2}, \ldots, x_{n}\right)=\frac{f \Theta \mid X_{1}, X_{2}, \ldots, X_{n}\left(\theta, x_{1}, x_{2}, \ldots, x_{n}\right)}{\int f_{\Theta}(\theta) f_{X_{1}, X_{2}, \ldots, X_{n} \mid \Theta}\left(x_{1}, x_{2}, \ldots, x_{n} \mid \theta\right) d \theta}
$$

Where, $f_{\Theta, X_{1}, X_{2}, \ldots, X_{n}}\left(\theta, x_{1}, x_{2}, \ldots, x_{n}\right)$ is the joint probability density function of $\Theta$ and the observed data. Using rules of conditional probability, this joint probability 
density function can be written as:

$$
f_{\Theta, X_{1}, X_{2}, \ldots, X_{n}}\left(\theta, x_{1}, x_{2}, \ldots, x_{n}\right)=f_{\Theta}(\theta) f_{X_{1}, X_{2}, \ldots, X_{n} \mid \Theta}\left(x_{1}, x_{2}, \ldots, x_{n} \mid \theta\right)
$$

Hence the above equation (Equation 2.4) can then be re-written as the continuous form of Bayes' equation:

$$
f_{\Theta \mid X_{1}, X_{2}, \ldots, X_{n}}\left(\theta \mid x_{1}, x_{2}, \ldots, x_{n}\right)=\frac{f_{\Theta}(\theta) f_{X_{1}, X_{2}, \ldots, X_{n} \mid \Theta}\left(x_{1}, x_{2}, \ldots, x_{n} \mid \theta\right)}{\int f_{\Theta}(\theta) f_{X_{1}, X_{2}, \ldots, X_{n} \mid \Theta}\left(x_{1}, x_{2}, \ldots, x_{n} \mid \theta\right) d \theta}
$$

$f_{X_{1}, X_{2}, \ldots, X_{n} \mid \Theta}\left(x_{1}, x_{2}, \ldots, x_{n} \mid \theta\right)$ is the called the likelihood function and is the probability density function for occurrence of the observed sample, given the parameter of distribution.

$f_{\Theta}(\theta)$ is called the prior density function and can be thought of as the way to express uncertainty about the distributional parameter $\theta$, before observing the data. While $f_{\Theta \mid X_{1}, X_{2}, \ldots, X_{n}}\left(\theta \mid x_{1}, x_{2}, \ldots, x_{n}\right)$ is called the posterior density function, the density function for the parameter as random variable $\Theta$, incorporating the knowledge of observed data [23]. A prior density function (or simply prior) can be chosen based on several criteria, as discussed next.

\subsubsection{Choice of Priors}

Prior density function can be chosen based on observed data or the assessment of experts. Such subjective priors are called informative priors. In conjunction with the observed data (via the likelihood function), they influence the posterior density function as obtained by Bayes' rule in Equation 2.5. As more data becomes available, the posterior obtained earlier can be used as the prior to obtain a more accurate posterior density function, in light of the new data.

In many cases (especially when there is no data or information available to construct a prior density function), it is desirable to have a prior density which plays minimal role in determining the posterior distribution. Such priors are called flat 
or non-informative priors. The most common way of obtaining a non-informative prior is by the principle of insufficient reason, where in absence of any information about the parameter, equal probabilities are assigned to all possible values of the parameter.

Another criterion for choosing a prior is computational complexity. To reduce computational complexity, often times priors are chosen in such a way that the resulting posterior density function is also of the same distributional family as the prior density. The parameters of posterior density function can be directly computed from the prior and the observed data by simple computations. Such priors are called conjugate priors [23].

Jeffreys compiled a collection of rules attempting to formalize a method for choosing prior for different scenarios $[80,38]$. In case of a finite parameter space, where the parameter can take $n$ values, Jeffreys used the principle of insufficient reason and assigned equal probability of $1 / n$ to each value of the parameter, thus the prior probability mass function was chosen as $p_{\Theta}(\theta)=1 / n$. Implying, in absence of any additional information, each of the parameter values are equally likely (hence a non-informative prior). In case of bounded continuous intervals (say $[a, b]$ ) for the parameter space, the prior density function is considered to be uniform density function over $[a, b]$ (again a non-informative prior). For the parameter of Bernoulli distribution, where the parameter space is $[0,1]$, the prior according to Jeffrey's rule is the non-informative uniform prior $f_{\Theta}(\theta)=1$.

For parameter space $(-\infty,+\infty)$, (e.g., in case of mean for normal distribution), he proposes to use a flat prior of a constant value, again keeping in mind the insufficient reason (though in this case, the prior will be an improper prior as it will not integrate to 1$)$. For parameters with parameter space $[0,+\infty)$ (e.g., the scale parameter of exponential and Weibull distributions), Jeffreys proposed a prior density function $f_{\Theta}(\theta) \propto 1 / \theta$. Invariance of the prior under power transformation of the 
scale parameter $\theta$, was cited as the reason for this prior. The guiding principle was that if there is a transformation of the parameter (random variable), the resulting prior should also express the same belief towards the transformed random variable as the original prior did towards untransformed random variable. In other words, any rule for determining the prior density should yield an equivalent result if applied to the transformed parameter. For example, if $\Lambda=\Theta^{2}$, then with $f_{\Theta}(\theta) \propto 1 / \theta, f_{\lambda}(\lambda)$ is still $\propto 1 / \lambda$.

\subsection{Epistemic Distributions}

The parameters of a stochastic dependability model may be determined from lifetime experiments, field failure data, maintenance logs or other sources of observed data. These data sources will provide a finite number of observations, leading to epistemic uncertainty in the estimates of model input parameters computed using the observed data. In many cases, the values of parameters may not even be based on observed data, but on expert guesses (another source of uncertainty).

To propagate the epistemic uncertainty in the input parameters of the stochastic aleatory model, the epistemic distribution (type and its parameters) of the model input parameter needs to be known. If the modeling expert constructing the stochastic dependability model has access to lifetime data, the type and parameters of the epistemic distribution of aleatory model input parameters as random variables, needs to be derived directly from that data. If the observed data is not directly accessible, the only information about epistemic uncertainty available to the modeler may be in the form of bounds or the confidence interval of the parameter value, from manufacturer datasheet or from literature summarizing lifetime studies. In such cases, we first derive the number of observations that would have been used to compute the point estimate or confidence intervals of the model input parameter as shown in Section 5.1.1, by inverting the relation between the confidence interval, point estimate 
and number of observations that would have been used to compute them. In this section, we derive the type and parameters of epistemic distribution of parameters of aleatory distribution, assuming that the number of observations used to compute their estimates is known (or can be computed).

\subsubsection{Determining Epistemic Distributions}

Let us say $r_{i}$ observed values of time to failure, $X$, of a component, were used to compute the point and interval estimates of parameter of its time to failure distribution (aleatory), $\Theta_{i}$ (which is the $i^{\text {th }}$ input parameter of the reliability model). The epistemic distribution of $\Theta_{i}$ can be obtained based on the knowledge of the aleatory distribution and the observed values of times to failure from the aleatory distribution, using the continuous form of Bayes' theorem [23].

If $X_{1}, X_{2}, \ldots, X_{r_{i}}$ be the independent and identically distributed random variables denoting the $r_{i}$ observed values of $X$, then the probability of these observations, given $\Theta_{i}=\theta_{i}$ (likelihood), is given by $f_{X_{1}, X_{2}, \ldots, X_{r_{i}} \mid \Theta_{i}}\left(x_{1}, x_{2}, \ldots, x_{r_{i}} \mid \theta_{i}\right)$. Applying the continuous form of Bayes' theorem, the epistemic density function for $\Theta_{i}$, given the set of observed values, can be obtained by:

$$
f_{\Theta_{i} \mid X_{1}, X_{2}, \ldots, X_{r_{i}}}\left(\theta_{i} \mid x_{1}, x_{2}, \ldots, x_{r_{i}}\right)=\frac{f_{\Theta_{i}}\left(\theta_{i}\right) f_{X_{1}, X_{2}, \ldots, X_{r_{i}} \mid \Theta_{i}}\left(x_{1}, x_{2}, \ldots, x_{r_{i}} \mid \theta_{i}\right)}{\int f_{\Theta_{i}}\left(\theta_{i}\right) f_{X_{1}, X_{2}, \ldots, X_{r_{i}} \mid \Theta_{i}}\left(x_{1}, x_{2}, \ldots, x_{r_{i}} \mid \theta_{i}\right) d \theta_{i}}
$$

In the above equation, $f_{\Theta_{i}}\left(\theta_{i}\right)$ is known as the prior density function [23]. The random deviates generated from the epistemic density derived in Equation 2.6 are used in our uncertainty propagation method as values of the parameters of the aleatory model. It is clear from Equation 2.6 that the epistemic density thus derived depends on the aleatory density and will be different for different aleatory densities. In the examples

discussed in this dissertation, exponential aleatory distribution (for times to failure and times taken by different recovery actions, job inter-arrival and service completion 
times, response times etc.) and Bernoulli aleatory distribution (for ability to detect or provide coverage of faults) are assumed. The form and parameters of epistemic distributions for parameters of exponential and Bernoulli aleatory distribution are derived next.

\subsubsection{Epistemic Distribution: Exponential Aleatory Distribution}

If the time to failure, $X$, is exponentially distributed with rate parameter $\Theta_{i}$, the $i^{t h}$ parameter in the reliability model of a system, then the random variable $S$, such that $S=\sum_{i=1}^{r_{i}} X_{i}$, will have an $r_{i}$-stage Erlang distribution with parameter $\theta_{i}$. Therefore, the probability density function of $S$, given $\Theta_{i}=\theta_{i}$, is:

$$
f_{S \mid \Theta_{i}}\left(s \mid \theta_{i}\right)=\frac{\theta_{i}^{r_{i}} s^{r_{i}-1} e^{-\theta_{i} s}}{\left(r_{i}-1\right) !}
$$

Then, applying Bayes' theorem as in Equation 2.6, the $p d f$ of $\Theta_{i}$, given $S=s$, will be given by:

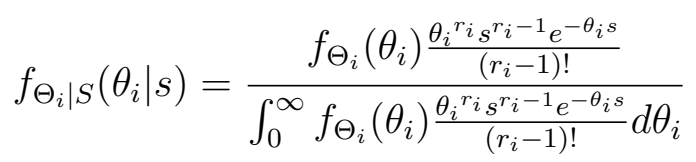

Assuming, Jeffreys' prior of $\Theta_{i}$, as $f_{\Theta_{i}}\left(\theta_{i}\right)=s / \theta_{i}$ [80], the above equation can be written as:

$$
\begin{aligned}
f_{\Theta_{i} \mid S}\left(\theta_{i} \mid s\right) & =\frac{\frac{s}{\theta} \frac{\theta_{i}{ }^{r} s^{r_{i}-1} e^{-\theta_{i} s}}{\left(r_{i}-1\right) !}}{\int_{0}^{\infty} \frac{s}{\theta} \frac{\theta_{i}^{r_{i}} s^{r_{i}-1} e^{-\theta_{i} s}}{\left(r_{i}-1\right) !} d \theta_{i}} \\
& =\frac{\frac{s^{r_{i}} \theta^{r_{i}-1} e^{-\theta s}}{r-1}}{\frac{s^{r}}{(r-1) !} \int_{0}^{\infty} \theta^{r-1} e^{-\theta s} d \theta}
\end{aligned}
$$

Using the result $\int_{0}^{\infty} x^{n} e^{-a x} d x=(n !) / a^{n+1}$, where $n$ is a non-negative integer and $a>0$, it can be seen in Equation 2.10 that the $p d f f_{\Theta_{i} \mid S}\left(\theta_{i} \mid s\right)$ results in the $p d f$ of 
$r_{i}-$ stage Erlang distribution with rate parameter $s$.

$$
\begin{aligned}
f_{\Theta_{i} \mid S}\left(\theta_{i} \mid s\right) & =\frac{\frac{s^{r} \theta^{r-1} e^{-\theta s}}{(r-1) !}}{\frac{s^{r}}{(r-1) !} \frac{(r-1) !}{s^{r}}} \\
& =\frac{\theta_{i}{ }^{r_{i}-1} s^{r_{i}} e^{-\theta_{i} s}}{\left(r_{i}-1\right) !}=\operatorname{Erlang} p d f\left(r_{i} ; s\right)
\end{aligned}
$$

This provides the epistemic probability density function for the rate parameter $\Theta_{i}$, when the aleatory distribution is exponential.

Extending Equations 2.7 and 2.10 to determine the epistemic distribution for the rate parameter of Erlang aleatory distribution will be trivial as the sum of Erlang distributed random variables $\left(S=\sum_{i=1}^{r} X_{i}\right)$ will still be Erlang distributed. An approximate epistemic $p d f$ for the parameters of Weibull aleatory distribution has been provided in [80], however it is not easy to sample from. If the epistemic distribution cannot be easily derived, a normal epistemic distribution may be assumed, based on the Central Limit Theorem, as an approximation.

\subsubsection{Epistemic Distribution: Bernoulli Aleatory Distribution}

Assume that the $j^{\text {th }}$ parameter $\theta_{j}$, of the reliability model, is a coverage parameter (say, the probability of successfully handling a fault) derived from a fault injection experiment, introducing $r_{j}$ faults. Given the probability of successfully covering a fault being $\theta_{j}$, the random variable $Y_{j}$ denoting the number of successfully covered faults out of $r_{j}$ faults injected, will have a binomial distribution with the probability mass function $(p m f)$ given by Equation 2.11.

$$
p_{Y_{j} \mid \Theta_{j}}\left(y_{j} \mid \theta_{j}\right)=\left(\begin{array}{c}
r_{j} \\
y_{j}
\end{array}\right) \theta_{j}^{y_{j}}\left(1-\theta_{j}\right)^{r_{j}-y_{j}}
$$

Then, based on Equation 2.6, the $p d f, f_{\Theta_{j} \mid Y_{j}}\left(\theta_{j} \mid y_{j}\right)$ can be derived as: 


$$
f_{\Theta_{j} \mid Y_{j}}\left(\theta_{j} \mid y_{j}\right)=\frac{f_{\Theta_{j}}\left(\theta_{j}\right)\left(\begin{array}{c}
r_{j} \\
y_{j}
\end{array}\right) \theta_{j}^{y_{j}}\left(1-\theta_{j}\right)^{r_{j}-y_{j}}}{\int_{0}^{\infty} f_{\Theta_{j}}\left(\theta_{j}\right)\left(\begin{array}{c}
r_{j} \\
y_{j}
\end{array}\right) \theta_{j}^{y_{j}}\left(1-\theta_{j}\right)^{r_{j}-y_{j}} d \theta_{j}}
$$

Choosing a non-informative prior (which is also Jeffreys' prior in this case [80]) of $f_{\Theta_{j}}\left(\theta_{j}\right)=1$, the above equation can be written as:

$$
\begin{aligned}
f_{\Theta_{j} \mid Y_{j}}\left(\theta_{j} \mid y_{j}\right) & =\frac{\left(\begin{array}{l}
r_{j} \\
y_{j}
\end{array}\right) \theta_{j} y_{j}\left(1-\theta_{j}\right)^{r_{j}-y_{j}}}{\int_{0}^{\infty}\left(\begin{array}{l}
r_{j} \\
y_{j}
\end{array}\right) \theta_{j}^{y_{j}}\left(1-\theta_{j}\right)^{r_{j}-y_{j}} d \theta_{j}} \\
& =\frac{\theta_{j}^{y_{j}}\left(1-\theta_{j}\right)^{r_{j}-y_{j}}}{\int_{0}^{1} \theta_{j}^{y_{j}}\left(1-\theta_{j}\right)^{r_{j}-y_{j}} d \theta_{j}}
\end{aligned}
$$

Using the result that for $x>0, y>0$, the integral

$$
\int_{0}^{1} t^{x-1}(1-t)^{y-1} d t=\Gamma(x) \Gamma(y) / \Gamma(x+y)
$$

the beta function $B(x, y)$, the integral in the denominator of Equation 2.13, $\int_{0}^{1} \theta_{j}^{y_{j}}\left(1-\theta_{j}\right)^{r_{j}-y_{j}} d \theta_{j}$ results in the beta function

$$
B\left(y_{j}+1, r_{j}-y_{j}+1\right)=\Gamma\left(y_{j}+1\right) \Gamma\left(r_{j}-y_{j}+1\right) / \Gamma\left(r_{j}+2\right)
$$

The $p d f f_{\Theta_{j} \mid Y_{j}}\left(\theta_{j} \mid y_{j}\right)$ then results in the beta density function with parameters $y_{j}+1$ and $r_{j}-y_{j}+1$ as shown below:

$$
\begin{aligned}
f_{\Theta_{j} \mid Y_{j}}\left(\theta_{j} \mid y_{j}\right) & =\frac{\theta_{j}^{y_{j}}\left(1-\theta_{j}\right)^{r_{j}-y_{j}}}{\int_{0}^{1} \theta_{j}^{y_{j}}\left(1-\theta_{j}\right)^{r_{j}-y_{j}} d \theta_{j}} \\
& =\frac{\theta_{j}^{y_{j}}\left(1-\theta_{j}\right)^{r_{j}-y_{j}}}{\frac{\Gamma\left(y_{j}+1\right) \Gamma\left(r_{j}-y_{j}+1\right)}{\Gamma\left(r_{j}+2\right)}} \\
& =\underbrace{\frac{\Gamma\left(r_{j}+2\right)}{\Gamma\left(y_{j}+1\right) \Gamma\left(r_{j}-y_{j}+1\right)} \theta^{y_{j}}\left(1-\theta_{j}\right)^{r_{j}-y_{j}}}_{\text {beta pdf(yjo }\left(y_{j}+r_{j}-y_{j}+1\right)}
\end{aligned}
$$




\subsection{Aleatory Vs. Epistemic Independence}

As seen in Section 2.1, the aleatory dependability models can range from simple non-state-space models which assume independence between events in the aleatory model (e.g. independence of component failures and repairs) to more complex state space models like Markov chains, semi-Markov and Markov regenerative processes which capture dependency between events or stochastic Petri nets to capture large state space $[8,56,24,4,86,31]$.

These models are solved at fixed values of the input parameters, however, in this dissertation, we consider the input parameters of the aleatory model to be random variables and propagate the uncertainty in the input parameters through the aleatory model. These epistemic random variables are often assumed to be independent for ease of uncertainty propagation by using marginal $p d f \mathrm{~s}$ of the input parameters as random variables, in place of joint $p d f \mathrm{~s}$. In real life, there may be dependencies between them, originating from the fact that they may be obtained from a common data or information source. In other words, some input parameters of the model may be correlated. Ignoring correlations between parameters can lead to errors and biases in the output metrics, depending on the degree of correlation and the parameters which are correlated. If the correlation is not very strong or if the model output is not very sensitive to the correlated parameters, the distribution

of the model output may not be affected in a significant way $[81,12]$. It has been argued that the dependency between model parameters arising out of commonality in the data or information source can be more intuitively captured by rank correlations rather than direct correlation between parameters $[28,35,30]$. Widely used distribution free methods of imposing rank correlation between parameter values, like the one proposed by Iman and Conover [35] can be used to take into account the epistemic dependence between model input parameters. It should be noted that 
assuming epistemic independence between the model input parameters as random variables, does not rule out considering dependency of any kind between events in the aleatory model (e.g., dependency between failure or repair events of components or dependency between failure modes of components). Dependence can always be allowed in the aleatory model via Markov chains, stochastic Petri nets or other state space models $[56,8,4,86]$, even when independence is assumed among the epistemic variables. Table 2.1 summarizes the differences between dependence in the aleatory model and the epistemic dependence between model input parameters as random variables.

Table 2.1: Epistemic Dependence Vs. Dependence in Aleatory Model

\begin{tabular}{|l|l|l|}
\hline & Epistemic Dependence & $\begin{array}{l}\text { Dependence in Aleatory } \\
\text { model }\end{array}$ \\
\hline $\begin{array}{l}\text { Dependence } \\
\text { Between }\end{array}$ & $\begin{array}{l}\text { Model input parameters as } \\
\text { random variables }\end{array}$ & $\begin{array}{l}\text { Events in the aleatory } \\
\text { model of the system }\end{array}$ \\
\hline $\begin{array}{l}\text { Reason for } \\
\text { dence }\end{array}$ & $\begin{array}{l}\text { Common source of data } \\
\text { used to compute parame- } \\
\text { ter values; Parameter values } \\
\text { guessed at by the same ex- } \\
\text { pert }\end{array}$ & $\begin{array}{l}\text { Failure or repair events of } \\
\text { components dependent on } \\
\text { the system; Failure modes } \\
\text { of components dependent } \\
\text { on each other or on state of } \\
\text { the system }\end{array}$ \\
\hline $\begin{array}{l}\text { Accounting } \\
\text { for Depen- } \\
\text { dence }\end{array}$ & $\begin{array}{l}\text { Use joint pdf of all model } \\
\text { input parameters; Employ } \\
\text { methods like Iman-Conover } \\
\text { method [35] to introduce } \\
\text { correlation between sam- } \\
\text { pled values from marginals }\end{array}$ & $\begin{array}{l}\text { Use state-space models like } \\
\text { Markov chains, stochas- } \\
\text { tic Petri nets/stochastic } \\
\text { reward nets. Hierarchical } \\
\text { models may also be used } \\
\text { [56, 8, 4, 86] }\end{array}$ \\
\hline
\end{tabular}




\section{3}

\section{Closed-form Approach for Epistemic Uncertainty Propagation}

In this chapter, we present a closed-form approach for propagation of epistemic uncertainty through aleatory models with closed-form expressions of model output. Method for deriving closed form expressions of CDF, expected value and variance of reliability, due to epistemic uncertainty in input parameter values, is discussed. Closed-form expressions for the distribution function, expected value and variance of reliability, are derived for several systems for a range of model output metrics. Limiting behavior of the CDF, expected value and variance of model output metric, is also studied ${ }^{1}$.

\subsection{Uncertainty Propagation}

Due to the epistemic uncertainties, the input parameters of a stochastic model can be considered a random vector. Therefore, the model output metric, can be considered a random variable that is a function of these input random variables. If ran-

dom variables $\left\{\Theta_{i}, i=1,2, \ldots, l\right\}$ are the set of $l$ input parameters of the aleatory

${ }^{1}$ Adapted from [53] 
model as random variables, the output measure $M$, can be viewed as a random variable (function) $g$ of the $l$ input parameters as $M=g\left(\Theta_{1}, \Theta_{2}, \ldots, \Theta_{l}\right)$. Due to the uncertainty associated with the model parameters, computing the reliability at specific parameter values can be seen as computing the conditional reliability $M\left(\Theta_{1}=\theta_{1}, \Theta_{2}=\theta_{2}, \ldots, \Theta_{l}=\theta_{l}\right)$ (denoted by $M($.$) in Equation 3.1). Applying the$ theorem of total probability [86], this can be unconditioned to compute the distribution of reliability via the joint epistemic density $f_{\Theta_{1}, \Theta_{2}, \ldots, \Theta_{l}}\left(\theta_{1}, \theta_{2}, \ldots, \theta_{l}\right)$ of the input parameters as random variables (denoted by $f($.$) in Equation 3.1).$

$$
F_{M}(m)=\int \ldots \int I(M \leq m) f(.) d \theta_{1} \ldots d \theta_{l}
$$

where $I(\xi)$ is the indicator variable of the event $\xi$. The unconditional expected value of model output $M$, can be computed as:

$$
E[M]=\int \ldots \int M(.) f(.) d \theta_{1} \ldots d \theta_{l}
$$

Similarly, the second moment of $M, E\left[M^{2}\right]$ can be computed, as:

$$
E\left[M^{2}\right]=\int \ldots \int(M(.))^{2} f(.) d \theta_{1} \ldots d \theta_{l}
$$

With the second moment and the expected value, the variance of model output metric, $\operatorname{Var}[M]$, can be computed using the relation $\operatorname{Var}[Y]=E\left[Y^{2}\right]-(E[Y])^{2}$, where $Y$ is a random variable [86]. When the aleatory model output is the reliability of a system at time $t, R(t)$, the unconditional expected value of the model output metric, as computed in Equation 3.2, has been termed as survivability, by Singpurwalla [80].

For simple aleatory models which can be solved analytically, a closed form expression of model output in terms of the model input parameters, can be obtained - In such cases, the above integrations can theoretically, be directly performed on 
the expression of model output, to propagate the epistemic uncertainty. For simpler expressions of the model output, the integration can be performed analytically, while for more complex expressions of reliability, numerical integration [63] may be performed. However, the task of evaluating these integrals quickly becomes intractable for complex expressions of model output or for larger number of model input parameters. Apart from the computational problem, the joint epistemic density of all the aleatory model input parameters as random variables, also needs to be specified. The problem becomes somewhat simpler if the epistemic random variables can be assumed to be independent, as the joint probability density functions can then be factored into the product of marginals. It should be noted that assuming epistemic independence between model parameters as random variables does not preclude considering dependency of all kinds between events in the aleatory reliability model, as explained in Section 2.4 (state-space models [86] can still be used to capture dependency between events, as usual).

In this chapter, propagation of parametric epistemic uncertainty through analytic integration of closed-form expression of the aleatory model output is considered. In the examples discussed here, the model parameters are considered to be independent.

Clearly, for this approach, the epistemic distributions of each of the parameters need to be determined or known first. Determination of epistemic distribution of aleatory model input parameters as random variables, from observed data and the knowledge of aleatory distributions, is discussed in Section 2.3.1 and is reproduced here for ease of reference in the later parts of this chapter.

The epistemic $p d f$ for the rate parameter of exponential aleatory distribution as random variable, $\Theta_{i}$, is given by:

$$
f_{\Theta_{i} \mid S}\left(\theta_{i} \mid s\right)=\frac{\theta_{i}^{r_{i}-1} s^{r_{i}} e^{-\theta_{i} s}}{\left(r_{i}-1\right) !}=\operatorname{Erlang} p d f\left(r_{i} ; s\right)
$$

where, $s$ is the value of total time on test random variable $S$ and $r_{i}$ is the number of 
observations used to compute the point estimate of $\theta_{i}$.

The epistemic $p d f$ of the probability parameter of Bernoulli aleatory distribution as random variable, $\Theta_{j}$, is given by:

$$
\begin{aligned}
f_{\Theta_{j} \mid Y_{j}}\left(\theta_{j} \mid y_{j}\right) & =\frac{\left(\begin{array}{l}
r_{j} \\
y_{j}
\end{array}\right) \theta_{j}{ }^{y_{j}}\left(1-\theta_{j}\right)^{r_{j}-y_{j}}}{\int_{0}^{\infty}\left(\begin{array}{l}
r_{j} \\
y_{j}
\end{array}\right) \theta_{j}^{y_{j}}\left(1-\theta_{j}\right)^{r_{j}-y_{j}} d \theta_{j}} \\
& =\underbrace{\frac{\Gamma\left(r_{j}+2\right)}{\Gamma\left(y_{j}+1\right) \Gamma\left(r_{j}-y_{j}+1\right)} \theta_{j}^{y_{j}}\left(1-\theta_{j}\right)^{r_{j}-y_{j}}}_{\text {beta pdf }\left(y_{j}+1 ; r_{j}-y_{j}+1\right)}
\end{aligned}
$$

where, $r_{j}$ is the number of faults injected in the fault injection experiment used to derive the point estimate of $\theta_{j}$ and the random variable $Y_{j}$ denotes the number of successfully covered faults out of $r_{j}$ faults injected.

Using the $p d f \mathrm{~s}$ in Equations 3.4 and 3.5, we perform closed-form epistemic uncertainty propagation through several aleatory models, next.

\subsection{Reliability of a Single Component System}

Reliability of a single component system at time $t$, when the time to failure of the component follows the exponential distribution with parameter $\lambda$, is given by, $R(t)=e^{-\lambda t}[86]$. In this section, we discuss obtaining the distribution, expectation and variance of reliability of such a single component system.

\subsubsection{Distribution of Reliability}

Due to the epistemic uncertainty in parameter $\lambda$, the reliability $R(t)$, computed at a fixed value of $\lambda$, can be seen to be conditioned on the value of $\lambda$ used. If the point estimate $\hat{\lambda}$ of parameter $\lambda$, were computed from $r$ observations of times to failure, then applying the theorem of total probability and using Equations 3.1 and 3.4, the 
cumulative distribution function $(\mathrm{CDF})$ of reliability at time $t$ can be computed as shown below.

$$
F_{R(t)}(p)=\int_{0}^{\infty} I(R(t) \leq p) \frac{\lambda^{r-1} s^{r} e^{-\lambda s}}{(r-1) !} d \lambda
$$

As $I($.$) is the indicator function, the above integral is non-zero only for values of$ $\lambda$ for which $R(t) \leq p$. Since $R(t)=e^{-\lambda t}$, it can be shown that the integral will be non-zero for $\lambda \geq \lambda_{a}$, such that $\lambda_{a}=-\ln p / t$. Using the expression for CDF of an Erlang distributed random variable [86] and knowing that $s=r / \hat{\lambda}$, the above equation reduces to:

$$
\begin{aligned}
F_{R(t)}(p) & =\int_{\lambda_{a}}^{\infty} \frac{\lambda^{r-1} s^{r} e^{-\lambda s}}{(r-1) !} d \lambda=1-\underbrace{\int_{0}^{\lambda_{a}} \frac{\lambda^{r-1} s^{r} e^{-\lambda s}}{(r-1) !} d \lambda}_{\text {Erlang } C D F} \\
& =\sum_{i=0}^{r-1} e^{-s \lambda_{a}} \frac{\left(s \lambda_{a}\right)^{i}}{i !} \\
& =\sum_{i=0}^{r-1} e^{\frac{r \ln p}{\hat{\lambda} t}} \frac{\left(\frac{-r \ln p}{\hat{\lambda} t}\right)^{i}}{i !}
\end{aligned}
$$

At any $t$ the reliability $R(t)$ of the system will have a distribution given by Equation 3.7 and hence will have an expected value, a variance and a confidence interval. Figure 3.1, shows the distribution of reliability, $F_{R(t)}(p)$, at different values of $t$. As $t$ increases, the CDF shifts to the left (i.e., the reliability tends to 0 , as $t$ increases, as expected). In this figure, the value of $\hat{\lambda}$ is chosen to be $5.7078 \times$ $10^{-5}$ per $h r .$, corresponding to an MTTF of 17,520 hrs., used for failure of software in [82] and number of observations, $r$, is chosen to be 10 .

Next we discuss the limiting behavior of this distribution as $r$ is varied. As provided by the Central Limit Theorem (CLT) [86], for very large values of $r$, the Erlang distribution for $\Lambda$ as derived in Equation 3.4 will tend to a normal distribution with mean $\mu_{\text {Normal }}=n / s=\hat{\lambda}$ and standard deviation $\sigma=\sqrt{r} / s=\hat{\lambda} / \sqrt{r}$. As 


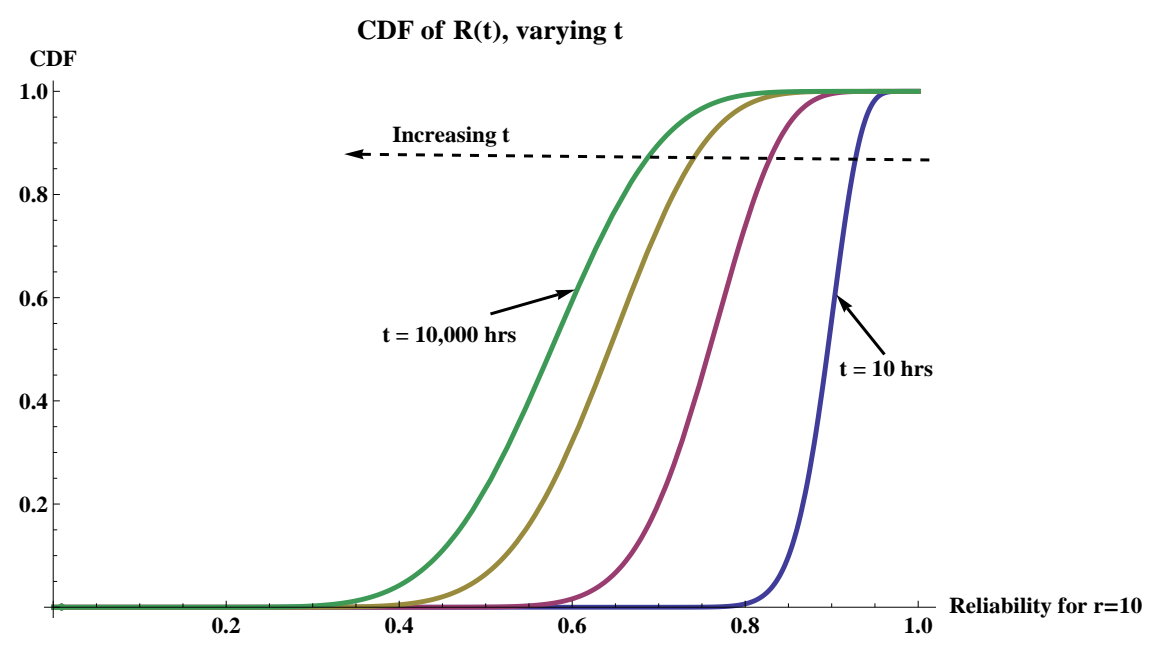

Figure 3.1: CDF of Reliability of a single-component system, for $r=10$

$r \rightarrow \infty, \sigma \rightarrow 0$ and the normal pdf tends to Dirac-delta function [60]. Hence the CDF of $\Lambda$ tends to Heaviside step function (or unit step function) $H[\lambda-\hat{\lambda}]$ [60], such that, for $\lambda \geq \hat{\lambda}$, the CDF evaluates to 1 and for all other values of $\lambda$, it evaluates to 0 . In other words, the only possible value of $\lambda$ as $r \rightarrow \infty$ is $\hat{\lambda}$, which being an unbiased estimate, is the true value of $\lambda$. Therefore, the only value of $R(t)$ possible as $r \rightarrow \infty$ is $e^{-\hat{\lambda} t}$ and the CDF of reliability, $F_{R(t)}(p)$ tends to Heaviside step function $H\left[p-e^{-\hat{\lambda} t}\right]$. Figure 3.2 shows the CDF of reliability, $R(t)$, at time $t=5000$ hours, as the number of observations $r$, is varied from 10 to 1000 . It can be seen clearly that as $r$ increases, the CDF tends to the step function. The value of $\hat{\lambda}$ is chosen as earlier to be $5.7078 \times 10^{-5}$ per hr.

\subsubsection{Expected Reliability}

If the point estimate $\hat{\lambda}$ of parameter $\lambda$, were computed from $r$ observations of times to failure, then using Equations 3.2 and 3.4, the unconditional expected reliability 


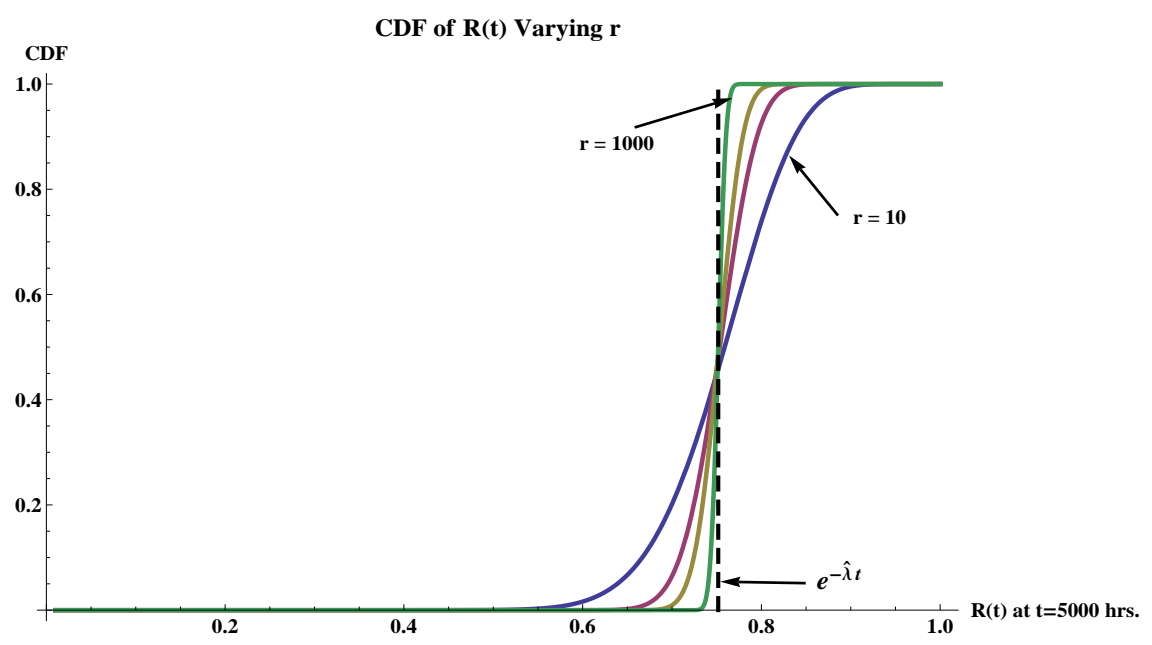

Figure 3.2: CDF of reliability of a single-component system at $t=5000$ hours

at time $t$ can be computed as:

$$
\begin{aligned}
E[R(t)] & =\int_{0}^{\infty} e^{-\lambda t} \cdot \underbrace{\frac{\lambda^{r-1} s^{r} e^{-\lambda s}}{(r-1) !}}_{\text {Erlang } p d f} d \lambda \\
& =\left(\frac{s}{s+t}\right)^{r}=\left(\frac{1}{1+\hat{\lambda} t / r}\right)^{r}
\end{aligned}
$$

The expression $(s /(s+t))^{r}$ in Equation 3.8 is of the form of complementary CDF of Pareto distribution with scale parameter $s$ and shape parameter $r$. The above equation makes use of the expression $\hat{\lambda}=r / s$, used to calculate the Maximum Likelihood Estimate (MLE) of $\lambda$. Using the identity $\lim _{h \rightarrow \infty}(1+1 / h)^{h}=e[86]$, the limiting value of unconditional expectation of reliability at time $t$, can be shown in Equation 3.9 to be $e^{-\hat{\lambda} t}$.

$$
\lim _{r \rightarrow \infty} E[R(t)]=\frac{1}{\lim _{r \rightarrow \infty}\left(1+\frac{\hat{\lambda} t}{r}\right)^{r}}=e^{-\hat{\lambda} t}
$$

Since $\hat{\lambda}$ is an unbiased estimate, it tends to the true value of $\lambda$, as $r \rightarrow \infty$. Figure 3.3 plots the expected value of $R(t)$, at $t=1000$, as a function of $r$. The value of 
$\hat{\lambda}$ is chosen to be $5.7078 \times 10^{-5}$ per $h r$., corresponding to an MTTF of 17, $520 \mathrm{hrs}$, used for failure of software in [82]. It can be seen that $E[R(t)]$ tends to $e^{-\hat{\lambda} t}$, as $r$ increases.

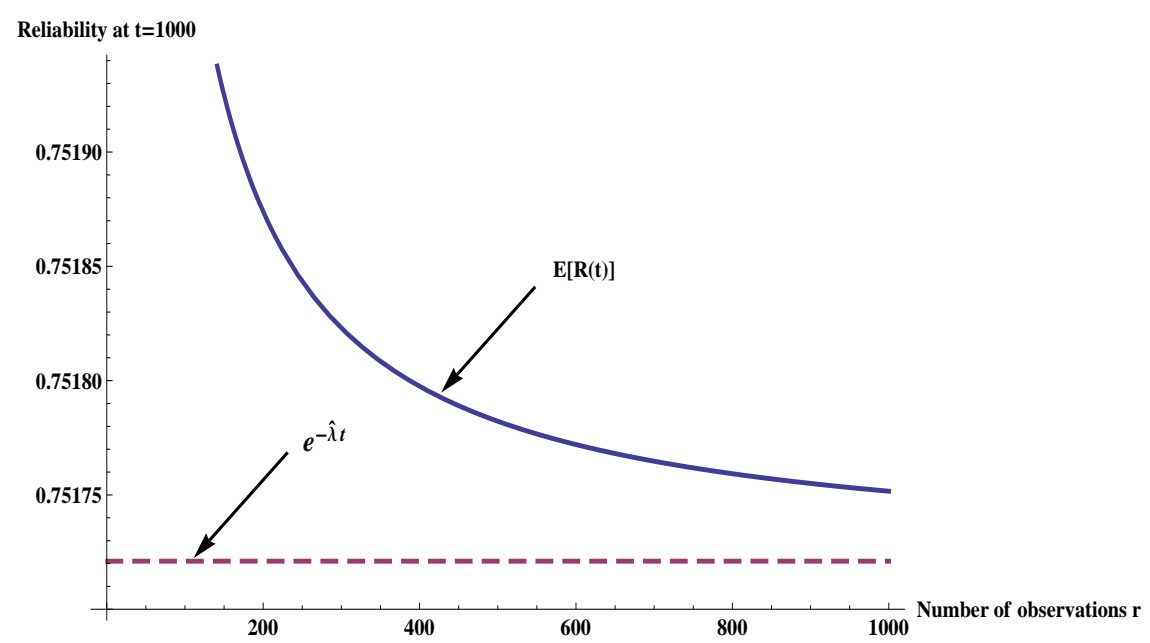

FigurE 3.3: Expected Reliability of a single-component system at $t=1000$ hours

\subsubsection{Variance of Reliability}

The relation $\operatorname{Var}[Y]=E\left[Y^{2}\right]-(E[Y])^{2}$, where $Y$ is a random variable, can be used to derive the variance of reliability at time $t, \operatorname{Var}[R(t)] . E\left[(R(t))^{2}\right]$ can be derived in a similar fashion as $E[R(t)]$ has been derived in Equation 3.8. Variance of reliability of a singe component system, at time $t$, is shown in Equation 3.10.

$$
\begin{aligned}
\operatorname{Var}[R(t)] & =\underbrace{\left(\frac{s}{s+2 t}\right)^{r}}_{E\left[(R(t))^{2}\right]}-\underbrace{\left(\frac{s}{s+t}\right)^{2 r}}_{E[R(t)]^{2}} \\
& =\left(\frac{1}{1+2 \hat{\lambda} t / r}\right)^{r}-\left(\frac{1}{1+\hat{\lambda} t / r}\right)^{2 r}
\end{aligned}
$$

It follows from Equation 3.10 that $\operatorname{Var}[R(t)] \rightarrow 0$ as $r \rightarrow \infty$. Using the same value of $\hat{\lambda}$ as in Section 3.2.2, Figure 3.4 shows the variance of reliability at time $t=1000$ hours. It is clear from the figure that $\operatorname{Var}[R(t)]$ tends to 0 as $r$ increases. 


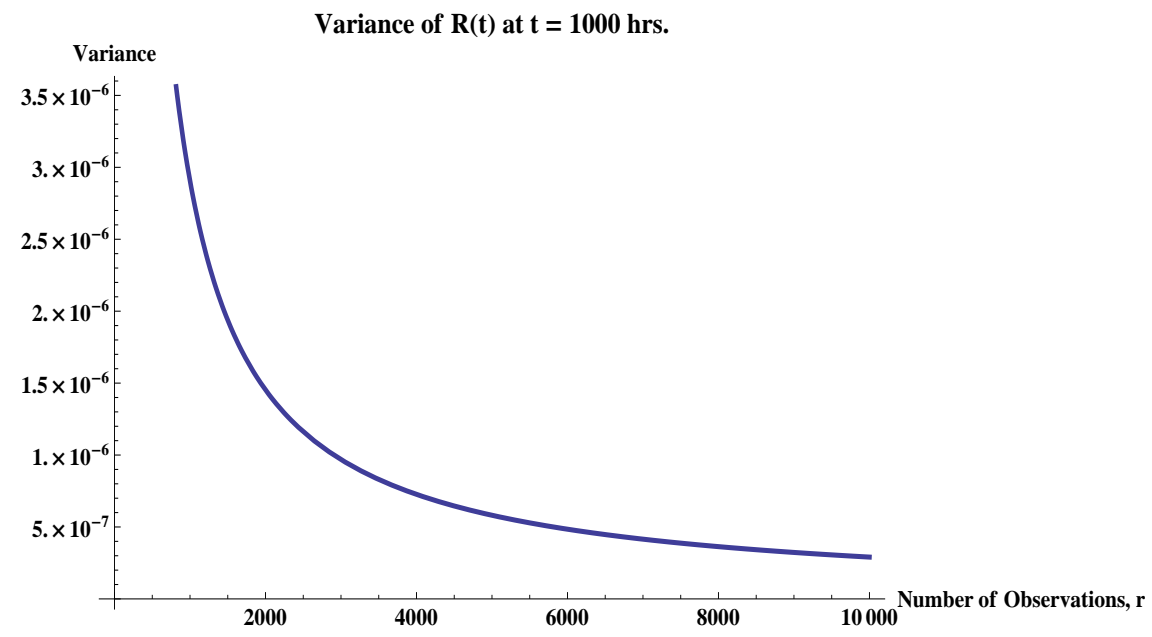

FiguRE 3.4: Variance of Reliability of a single-component system at $t=1000$ hours

\subsection{Series System Reliability}

For a system with $n$ identical components, each with independent and identically distributed times to failure, following the exponential distribution with parameter $\lambda$, the reliability $R(t)$ is given by $R(t)=e^{-n \lambda t}$. Building upon the distribution and expressions for the expected value and variance of reliability derived for a single component case in Section 3.2, expressions for the same can be derived for the $n$ component series system. Extending Equation 3.7, the distribution of reliability of an $n$-component series system can be derived as:

$$
\begin{aligned}
& F_{R(t)}(p)=\int_{\lambda_{a n}}^{\infty} \frac{\lambda^{r-1} s^{r} e^{-\lambda s}}{(r-1) !} d \lambda=1-\int_{0}^{\lambda_{a n}} \frac{\lambda^{r-1} s^{r} e^{-\lambda s}}{(r-1) !} d \lambda \\
& =1-\underbrace{\int_{C D F \text { or Incomplete gamma function }}^{\int_{0}^{\frac{-\ln p}{n t}} \frac{\lambda^{r-1} s^{r} e^{-\lambda s}}{(r-1) !} d \lambda}}_{\text {gamma }}
\end{aligned}
$$

where, $\lambda_{a n}$ is the value of $\lambda$, such that the reliability of an $n$-component series system $R(t)=e^{-n \lambda t} \geq p$. The limiting behavior of distribution as $r \rightarrow \infty$, seen in Section 3.2.1 will apply to the $n$-component series system as well, with the distribution tending to a Heaviside step function $H\left[R(t)-e^{-n \hat{\lambda} t}\right]$, as $r \rightarrow \infty$. 
The expected reliability for the series system can be derived as shown below:

$$
E\left[R_{n \text { series }}(t)\right]=\left(\frac{s}{s+n t}\right)^{r}=\left(\frac{1}{1+n \hat{\lambda} t / r}\right)^{r}
$$

It can be shown, similar to Equation 3.9, that as the number of observations, $r$, used to compute the rate parameter $\lambda$, tends to $\infty$, the expected value of reliability on $n$-component system will tend to $e^{-n \hat{\lambda} t}$.

The variance of reliability, due to the epistemic uncertainty in parameter $\lambda$ can be derived using the relation, $\operatorname{Var}[Y]=E\left[Y^{2}\right]-(E[y])^{2}$, where $Y$ is a random variable. The variance of reliability of an $n$-component series system is :

$$
\begin{aligned}
\operatorname{Var}\left[R_{\text {nseries }}(t)\right] & =\left(\frac{s}{s+2 n t}\right)^{r}-\left(\frac{s}{s+n t}\right)^{2 r} \\
& =\left(\frac{1}{1+2 n \hat{\lambda} t / r}\right)^{r}-\left(\frac{1}{1+n \hat{\lambda} t / r}\right)^{2 r}
\end{aligned}
$$

The limiting behavior of the variance, as $r \rightarrow \infty$ can be computed making use of the identity $\lim _{h \rightarrow+\text { infty }}(1+1 / h)^{h}=e$. I can be seen that as $r \rightarrow \infty, \operatorname{Var}[R(t)] \rightarrow$ $e^{-2 n \hat{\lambda} t}-e^{-2 n \hat{\lambda} t}=0$.

\subsubsection{Comparing Confidence Interval}

We compare the confidence interval of reliability of a series system obtained by using the value of reliability at appropriate percentile points from the distribution computed as in Equation 3.11, to the confidence interval of reliability of series system computed as explained by Sarkar in [74] and discussed in Equation 1.1.

Consider a series system with $n=10$ components, each with an exponentially distributed time to failure, with a failure rate $\lambda$ and the point estimate $\hat{\lambda}$ to be $5.7078 \times 10^{-5}$ per $h r$. (corresponding to an MTTF of 17, 520 hrs., used for failure of software in [82]). If the number of observed times to failure, used to compute the 
point estimate of failure rate were $r=50$, then the lower limit of $95 \%$ upper onesided confidence interval of reliability of the series system, at time $t=1000 \mathrm{hrs.}$, is given by $e^{-t n \hat{\lambda} \chi^{2}{ }_{2 r}(0.05) /(2 r)}$ as shown by Sarkar in [74], and is computed to be 0.4918 . Using Equation 3.11, the CDF of reliability of the series system is first generated and the value of reliability at the $5^{\text {th }}$ percentile point, 0.4925 , is chosen as the lower limit of $95 \%$ upper one-sided confidence interval of reliability of the series system, at time $t=1000$ hrs.. Thus both the method by Sarkar in [74] as well as the method explained in this chapter, provide almost the same values for the limits of the confidence interval.

However, the confidence interval of reliability of a series system can be obtained directly by the method explained by Sarkar in [74] whereas in the closed-form analytic method explained in this chapter, the confidence interval has to be obtained by first computing the distribution and then choosing the value of reliability from appropriate percentile points of the distribution. On the other hand, the method explained in [74] can only be used to compute the confidence intervals for reliability of a series system, while the method explained in this chapter can be used to obtain the distribution, expectation and variance of the aleatory model output for a wider range of systems.

\subsection{Reliability of a TMR System}

A triple modular redundant (TMR) system is considered to be operational as long two of its three components are operational [86]. The reliability of a TMR system, at time $t$, where all the three components have independent and identically distributed times to failure, following the exponential distribution with parameter $\lambda$, is given by:

$$
R(t)=3 e^{-2 \lambda t}-2 e^{-3 \lambda t}
$$




\subsubsection{Distribution of Reliability : TMR System}

The distribution of reliability of a TMR system due to the epistemic uncertainty in parameter $\lambda$ can be derived using Equations 3.1 and 3.4, as shown in Section 3.2.1. Equation 3.15 gives the CDF of reliability of a TMR system.

$$
F_{R(t)}(p)=\int_{0}^{\infty} I(R(t) \leq p) \frac{\lambda^{r-1} s^{r} e^{-\lambda s}}{(r-1) !} d \lambda
$$

The above integral is non-zero only for values of $\lambda$ for which $R(t) \leq p$. The inequality $3 e^{-2 \lambda t}-2 e^{-3 \lambda t}-p \leq 0$ is solved to obtain $\lambda_{\text {atmr }}$ such that, for $\lambda \geq \lambda_{\text {atmr }}, I(R(t) \leq$ $p)=1$. Using the CDF of an Erlang distributed random variable, Equation 3.15 then reduces to:

$$
\begin{aligned}
F_{R(t)}(p) & =\int_{0}^{\infty} I(R(t) \leq p) \frac{\lambda^{r-1} s^{r} e^{-\lambda s}}{(r-1) !} d \lambda \\
& =\int_{\lambda_{a t m r}}^{\infty} \frac{\lambda^{r-1} s^{r} e^{-\lambda s}}{(r-1) !} d \lambda=1-\underbrace{\int_{0}^{\lambda_{a t m r}} \frac{\lambda^{r-1} s^{r} e^{-\lambda s}}{(r-1) !} d \lambda}_{\text {Erlang } C D F} \\
& =\sum_{i=0}^{r-1} e^{-s \lambda_{a t m r}} \frac{\left(s \lambda_{a t m r}\right)^{i}}{i !}=\sum_{i=0}^{r-1} e^{\frac{-r \lambda_{a t m r}}{\hat{\lambda}}} \frac{\left(\frac{r \lambda_{a t m r}}{\hat{\lambda}}\right)^{i}}{i !}
\end{aligned}
$$

As discussed in Section 3.2.1, as the number of observations of times to failure, $r$, tends to $\infty, \hat{\lambda}$ tends to the true value of $\lambda$ and hence the distribution of $\Lambda$ is a Heaviside step function. It follows that the CDF of reliability of a TMR system, $F_{R(t)}(p)$, as $r \rightarrow \infty$, tends to a Heaviside step function, $H\left[p-\left(3 e^{-2 \hat{\lambda} t}-2 e^{-3 \hat{\lambda} t}\right)\right]$, with the step at $3 e^{-2 \hat{\lambda} t}-2 e^{-3 \hat{\lambda} t}$. Figure 3.5 shows the CDF of reliability of a TMR system, $R(t)$, at time $t=5000$ hours, as the number of observations $r$, is varied from 10 to 1000. It can be seen clearly that as $r$ increases, the CDF tends to the step function. The value of $\hat{\lambda}$ is chosen as earlier to be $5.7078 \times 10^{-5}$ per $h r$. . 


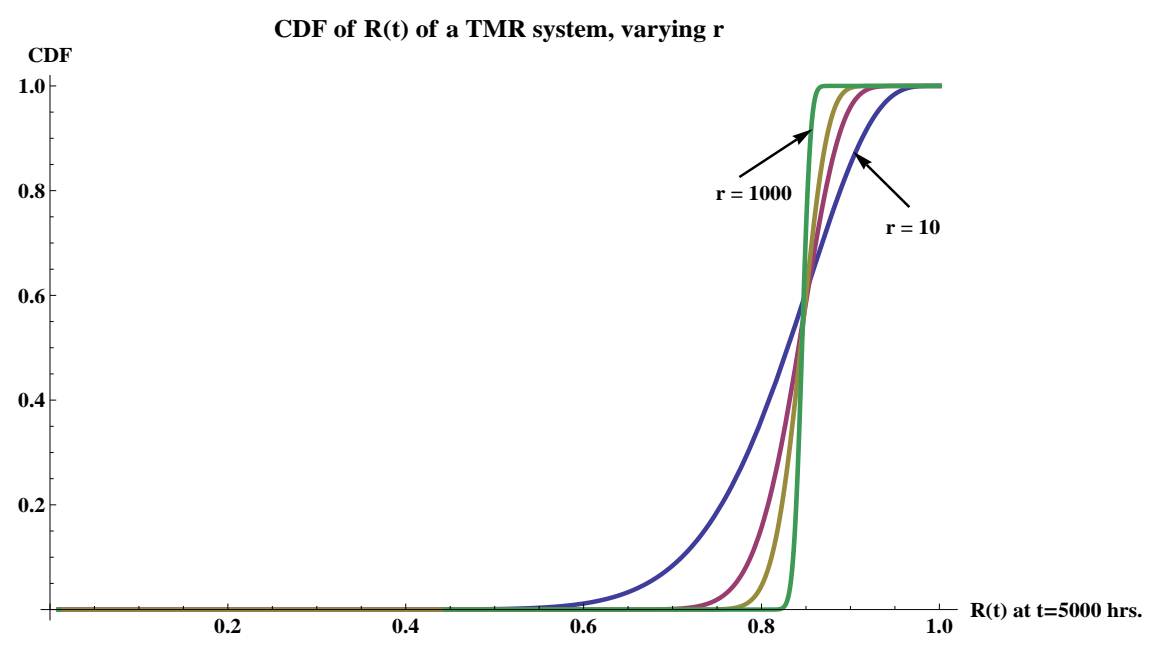

FiguRE 3.5: CDF of reliability of a TMR system at $\mathrm{t}=5000$ hours.

\subsubsection{Expected Reliability: TMR System}

Based on Equations 3.8 and 3.12, the unconditional expected reliability of a TMR system, at time $t$ can be computed as:

$$
\begin{aligned}
E[R(t)] & =\int_{0}^{\infty}\left(3 e^{-2 \lambda t}-2 e^{-3 \lambda t}\right) \cdot \underbrace{\frac{\lambda^{r-1} s^{r} e^{-\lambda s}}{(r-1) !}}_{\text {Erlang pdf }} d \lambda \\
& =3\left(\frac{s}{s+2 t}\right)^{r}-2\left(\frac{s}{s+3 t}\right)^{r} \\
& =3\left(\frac{1}{1+2 \hat{\lambda} t / r}\right)^{r}-2\left(\frac{1}{1+3 \hat{\lambda} t / r}\right)^{r}
\end{aligned}
$$

Using the identity $\lim _{h \rightarrow \infty}(1+1 / h)^{h}=e$, similar to Equation 3.9, it can be shown that as the number of observations $r \rightarrow \infty$, the value of unconditional expectation of reliability at time $t$ tends to $3 e^{-2 \hat{\lambda} t}-2 e^{-3 \hat{\lambda} t}$. Figure 3.6 plots the expected value of $R(t)$, at $t=1000$, as a function of $r$. The value of $\hat{\lambda}$ is chosen to be $5.7078 \times$ $10^{-5}$ per $h r$., as earlier. It can be seen that $E[R(t)]$ tends to $3 e^{-2 \hat{\lambda} t}-2 e^{-3 \hat{\lambda} t}$, as $r$ increases. 


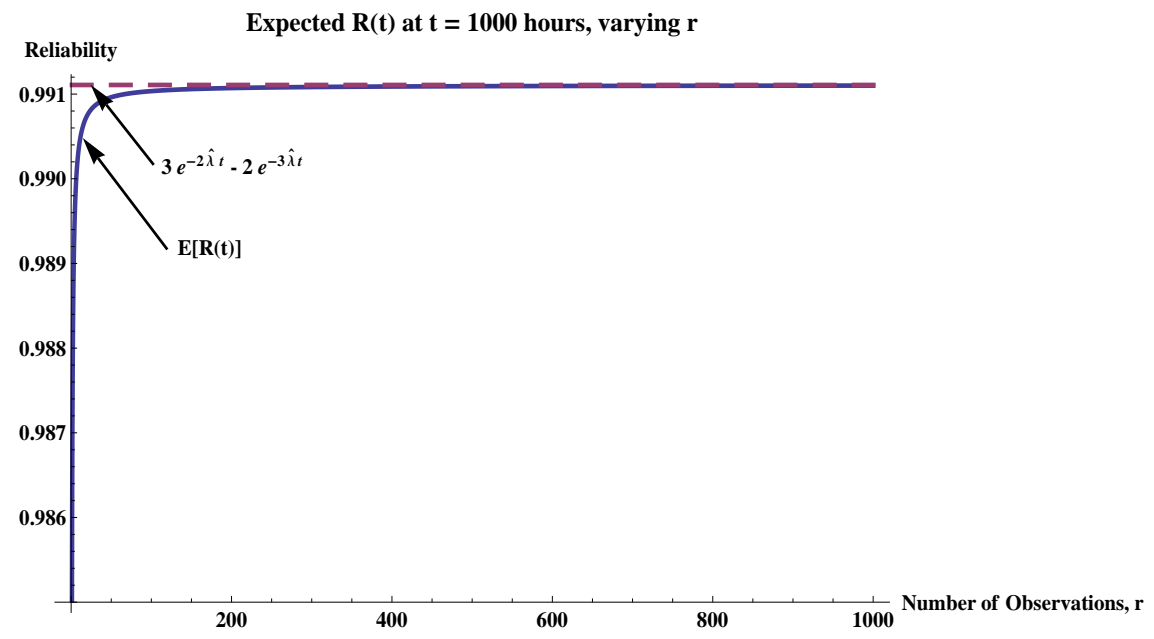

FIgURE 3.6: Expected Reliability of a TMR system at $\mathrm{t}=1000$ hours

\subsubsection{Variance of Reliability : TMR System}

Variance of reliability of a TMR system at time $t$, due to the epistemic uncertainty in the rate of component failure rate parameter $\lambda$, is derived by extending Equations 3.10 and 3.13 , as:

$$
\begin{aligned}
\operatorname{Var}[R(t)]= & \underbrace{9\left(\frac{s}{s+4 t}\right)^{r}-12\left(\frac{s}{s+5 t}\right)^{r}+4\left(\frac{s}{s+6 t}\right)^{r}}_{E\left[(R(t))^{2}\right]} \\
& -\underbrace{\left(3\left(\frac{s}{s+2 t}\right)^{r}-2\left(\frac{s}{s+3 t}\right)^{r}\right)^{2}}_{E[R(t)]^{2}} \\
= & 9\left(\frac{1}{1+4 \hat{\lambda} t / r}\right)^{r}-12\left(\frac{1}{1+5 \hat{\lambda} t / r}\right)^{r}+4\left(\frac{1}{1+6 \hat{\lambda} t / r}\right)^{r} \\
& -\left(3\left(\frac{1}{1+2 \hat{\lambda} t / r}\right)^{r}-2\left(\frac{1}{1+3 \hat{\lambda} t / r}\right)^{r}\right)^{2}
\end{aligned}
$$

It follows from Equation 3.19 that $\operatorname{Var}[R(t)] \rightarrow 0$ as $r \rightarrow+\infty$. Using the same value of $\hat{\lambda}$ as in Section 3.2.1, Figure 3.7 shows the variance of reliability at time $t=1000$ hours. It is clear from the figure that $\operatorname{Var}[R(t)]$ tends to 0 as $r$ increases. 


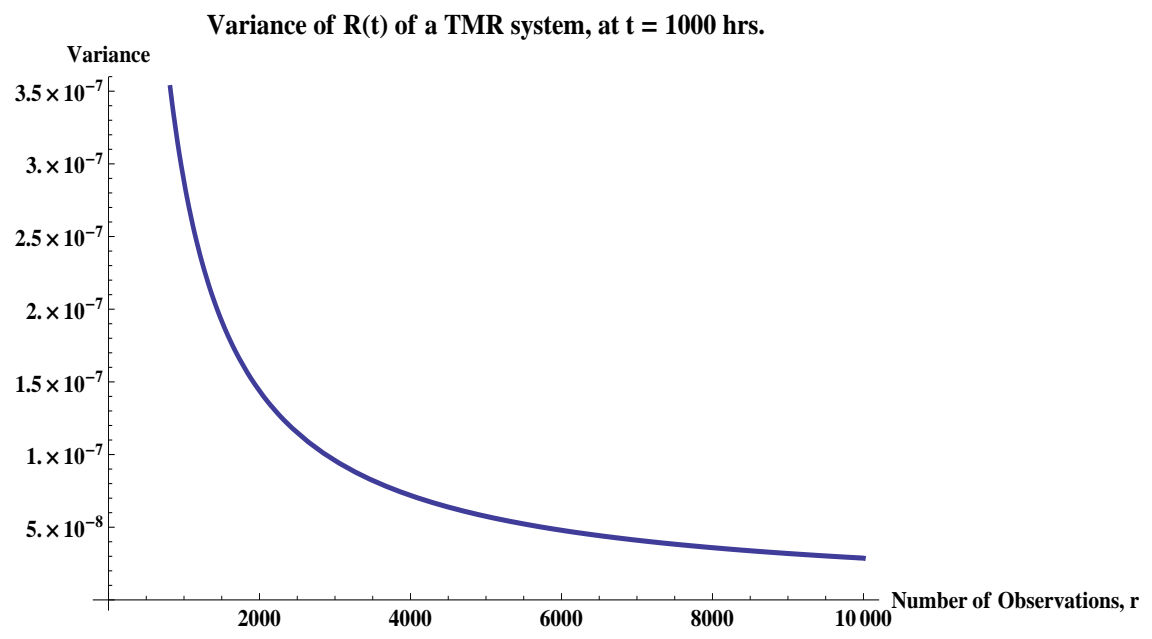

FiguRE 3.7: Variance of Reliability of a TMR system at $t=1000$ hours

\subsection{Reliability of a k-out-of-n System}

A $k-$ out $-o f-n$ system is considered to be operational as long as at least $k$ of the total $n$ components in the system are operational [86]. Consider a $k$ - out of $-n$ system, where each of the $n$ components has an independent and identically distributed time to failure, following the exponential distribution with parameter $\lambda$. The reliability $R(t)$ of such a system is given by:

$$
R(t)=\sum_{i=k}^{n}\left(\begin{array}{l}
n \\
i
\end{array}\right)\left(\begin{array}{l}
i-1 \\
k-1
\end{array}\right)(-1)^{i-k} e^{-i \lambda t}
$$

Since $e^{-i \lambda t}$ is the same expression as the reliability of an $i$-component series system, using the linearity property of expectation [86], the expected reliability of a $k-$ out - of $-n$ system can be derived based on Equation 3.12. As in the earlier sections, assume that the number of observations of times to failure of a component, used to compute the point estimate $\hat{\lambda}$, is $r$ and that the value of random variable $S$, denoting the sum of observed times to failure, is $s$. Equation 3.21 provides the expression for the expected reliability, $E[R(t)]$, of a $k-$ out - of $-n$ system with all 
components having independent and identically distributed times to failure.

$$
\begin{aligned}
E[R(t)] & =\sum_{i=k}^{n}\left(\begin{array}{l}
n \\
i
\end{array}\right)\left(\begin{array}{l}
i-1 \\
k-1
\end{array}\right)(-1)^{i-k}\left(\frac{s}{s+i t}\right)^{r} \\
& =\sum_{i=k}^{n}\left(\begin{array}{l}
n \\
i
\end{array}\right)\left(\begin{array}{l}
i-1 \\
k-1
\end{array}\right)(-1)^{i-k}\left(\frac{1}{1+i \hat{\lambda} t / r}\right)^{r}
\end{aligned}
$$

The limiting value of expected reliability of a $k-$ out $-o f-n$ system, as $r \rightarrow+\infty$ can easily be shown to be tending to the value of $R(t)$, (Equation 3.20), computed at

point estimate $\hat{\lambda}$, which, being an unbiased estimate, is also equal to the true value, as $r \rightarrow \infty$.

Since the components have independent failures, from Equation 3.20 it follows that the variance of reliability of a $k-$ out $-o f-n$ system, can be derived as:

$$
\begin{aligned}
\operatorname{Var}\left[R_{k o f n}(t)\right]= & \sum_{i=k}^{n}\left(\left(\begin{array}{c}
n \\
i
\end{array}\right)\left(\begin{array}{l}
i-1 \\
k-1
\end{array}\right)(-1)^{i-k}\right)^{2} \operatorname{Var}\left[e^{-i \lambda t}\right] \\
= & \sum_{i=k}^{n}\left(\left(\begin{array}{c}
n \\
i
\end{array}\right)\left(\begin{array}{l}
i-1 \\
k-1
\end{array}\right)\right)^{2} \\
& \left(\left(\frac{1}{1+2 i \hat{\lambda} t / r}\right)^{r}-\left(\frac{1}{1+i \hat{\lambda} t / r}\right)^{2 r}\right)
\end{aligned}
$$

The variance of the $k$ - out - of $-n$ system can be be shown to tend to 0 , as $r \rightarrow \infty$, as each of the terms in the summation in Equation 3.22 tend to 0, when $r \rightarrow \infty$.

The distribution of reliability of a $k-$ out $-o f-n$ system, due to the epistemic uncertainty in parameter $\lambda$ can be derived using Equations 3.1 and 3.4, as shown in 
Section 3.2. The CDF of reliability of a $k-$ out - of $-n$ system is given by:

$$
\begin{aligned}
F_{R_{k o f n}(t)}(p) & =\int_{0}^{\infty} I\left(R_{k o f n}(t) \leq p\right) \frac{\lambda^{r-1} s^{r} e^{-\lambda s}}{(r-1) !} d \lambda \\
& =\int_{\lambda_{\text {akofn }}}^{\infty} \frac{\lambda^{r-1} s^{r} e^{-\lambda s}}{(r-1) !} d \lambda \\
& =1-\underbrace{\int_{0}^{\lambda_{\text {akofn }}} \frac{\lambda^{r-1} s^{r} e^{-\lambda s}}{(r-1) !} d \lambda}_{\text {Erlang } C D F} \\
& =\sum_{i=0}^{r-1} e^{-s \lambda_{\text {akofn }} \frac{\left(s \lambda_{\text {akofn }}\right)^{i}}{i !}}
\end{aligned}
$$

where $\lambda_{\text {akofn }}$ is the value of $\lambda$ for which $R_{k o f n}(t)-p \leq 0$.

Using different values for $k$ and $n$, the above expressions can be used to compute the expected reliability and variance of reliability, due to epistemic uncertainty in failure rate parameter, for different series, parallel or k-out-of-n (also called N-modular redundant (NMR) [58]) systems.

\subsubsection{Variance of Parallel System Reliability}

We compare the variance of reliability of a parallel system computed by the method of closed-form analytic solution of the unconditioning integral 3.3, as discussed in this chapter to that obtained by the method explained by Coit in [16], which uses the linearity property of expectation and linear transformations of variance (assuming independent failures of the components). Based on Equation 3.22, the expression for variance of an $n$ component parallel system, where each component has an independent and identically distributed time to failure following the exponential distribution 
with parameter $\lambda$, is given by:

$$
\begin{aligned}
\operatorname{Var}\left[R_{\text {parallel }}(t)\right]= & \sum_{i=1}^{n}\left(\left(\begin{array}{c}
n \\
i
\end{array}\right)(-1)^{i-1}\right)^{2} \operatorname{Var}\left[e^{-i \lambda t}\right] \\
= & \sum_{i=1}^{n}\left(\left(\begin{array}{c}
n \\
i
\end{array}\right)\right)^{2} \\
& \left(\left(\frac{1}{1+2 i \hat{\lambda} t / r}\right)^{r}-\left(\frac{1}{1+i \hat{\lambda} t / r}\right)^{2 r}\right)
\end{aligned}
$$

The method explained in [16] computes the reliability of an $n$ component parallel system, with independent and identically distributed times to failure as:

$$
\operatorname{Var}\left[R_{\text {parallel }}(t)\right]=\left(\prod_{i=1}^{n}\left(\sigma^{2}+(1-\mu)^{2}\right)\right)-\prod_{i=1}^{n}(1-\mu)^{2}
$$

where, $\sigma^{2}$ is the variance of the reliability of a component and $\mu$ is the mean of the reliability of a component.

It should be noted that while Equation 3.24 (based on the closed-form analytic method explained in this chapter) uses the point estimate of parameter of time to failure distribution (aleatory) and the number of observations used to compute it (and these can be easily obtained from the observed failure data), Equation 3.25 (based on Coit's method [16]), requires the mean and variance of reliability of the component (which is not as straightforward to obtain). However, Equation 3.24 can be applied only when the time to failure of each component is exponentially distributed, while Equation 3.25 (from Coit's method) can be applied to any distribution of failure times of components. On the other hand the method explained in this chapter can be applied to compute the distribution, expectation and variance of the aleatory model output due to the epistemic uncertainties in the input parameters, for a much wider range of models (as can be seen in the examples in this chapter) than reliability 
models of just series-parallel systems (as possible with Coit's method explained in $[16])$.

\subsection{Duplex System with a Spare}

Consider a duplex system with a spare, where 2 components are initially in operation (active) and a third component is a warm spare (de-energized standby). Each active component has an exponentially distributed time to failure with parameter $\lambda$ (independent and identically distributed), while the standby component has an exponentially distributed time to failure with parameter $\mu$ (where, $\mu$ is usually expected to be less than $\lambda$ ). Upon failure of an active component, the standby component is brought into active operation and then follows the exponential failure law with pa-

rameter $\lambda$. The system is considered to be operational as long as 2 of the components are in active operation [86]. The reliability $R(t)$ of such a system is:

$$
R(t)=\left(2 \frac{\lambda}{\mu}+1\right) e^{-2 \lambda t}-2 \frac{\lambda}{\mu} e^{(-2 \lambda+\mu) t}
$$

While the epistemic random variables $(\Lambda$ and $M)$ are assumed to be independent for uncertainty propagation purposes, the aleatory model takes into account different failure rates of components in different states (active or standby).

\subsubsection{Expected Reliability : Duplex System with a Spare}

The expected reliability of a duplex system with a spare, taking into account the epistemic uncertainties in parameters $\lambda$ and $\mu$, can be derived based on Equation 3.2. Assume that the point estimate $\hat{\lambda}$ is computed using $r$ observations of times to failure and that the value of random variable representing sum of observed times to failure $S$, is $s$, while the point estimate $\hat{\mu}$ (of random variable $M$ corresponding to failure rate in standby mode) is derived using $u$ observations of times to failure 
of component in standby mode, with the value of sum of observed times to failure random variable $B$, being $b$. The expected reliability derived, is shown below in 3.27.

$$
\begin{aligned}
E[R(t)]= & \int_{0}^{\infty} \int_{0}^{\infty}\left(\left(2 \frac{\lambda}{\mu}+1\right) e^{-2 \lambda t}-2 \frac{\lambda}{\mu} e^{(-2 \lambda+\mu) t}\right) \underbrace{\frac{\lambda^{r-1} s^{r} e^{-\lambda s}}{(r-1) !} \underbrace{\frac{\mu^{u-1} b^{u} e^{-\mu b}}{(u-1) !}}_{\text {pdf of } M} d \lambda d \mu}_{\text {pdf of } \Lambda} \\
= & {\left[\left(\frac{s}{s+2 t}\right)^{r} \frac{1}{s+2 t}\left(\frac{b}{b+t}\right)^{u-1} \frac{(-2) b \times r}{u-1}\right] } \\
& +\left[\left(\frac{s}{s+2 t}\right)^{r} \frac{1}{s+2 t} \frac{2 b \times r}{u-1}\right]+\left(\frac{s}{s+2 t}\right)^{r}
\end{aligned}
$$

Using the number of observations $r$ and the point estimate $\hat{\lambda}$, the value $s$, of sum of observed times to failure random variable, $S$ can be expressed as, $s=r / \hat{\lambda}$. Similarly, $b$ can be expressed as $b=u / \hat{\mu}$. Equation 3.27 can then be re-written as:

$$
\begin{aligned}
E[R(t)]= & \underbrace{\left[\left(\frac{1}{1+2 \hat{\lambda} t / r}\right)^{r} \frac{1}{r / \hat{\lambda}+2 t}\left(\frac{1}{1+\hat{\mu} t / u}\right)^{u-1} \frac{(-2) u \times r}{\hat{\mu}(u-1)}\right]}_{I} \\
& +\underbrace{\left[\left(\frac{1}{1+2 \hat{\lambda} t / r}\right)^{r} \frac{1}{r / \hat{\lambda}+2 t} \frac{2 u \times r}{\hat{\mu}(u-1)}\right]}_{I I}+\underbrace{\left(\frac{1}{1+2 \hat{\lambda} t / r}\right)}_{I I I}
\end{aligned}
$$

It can be shown, similar to Equation 3.9, that as $r \rightarrow \infty$ and $u \rightarrow \infty$, the term in Equation 3.28 marked by $I$, tends to $-2 e^{-(2 \hat{\lambda}+\hat{\mu}) t} \hat{\lambda} / \hat{\mu}$, term $I I$ tends to $2 e^{-2 \hat{\lambda} t} \hat{\lambda} / \hat{\mu}$ and term marked by $I I I$ tends to $e^{-2 \hat{\lambda} t}$. Thus in the limiting case, the expected reliability of a duplex system with a spare, tends to the reliability evaluated at $\hat{\lambda}$ and $\hat{\mu}$ (which being unbiased estimates, are also the true values of $\lambda$ and $\mu$ as $r$ and $u$ tend to $\infty$, respectively). 
Figure 3.8 plots the expected reliability, $E[R(t)]$ of a duplex system with one spare, at time $t=1000$ hours. The value for $\hat{\lambda}$ is chosen to be $5.7078 \times 10^{-5} \mathrm{per} h r$. corresponding to an MTTF of 17,520 hours and $\hat{\mu}$ is chosen to be half of $\hat{\lambda}$. While Equation 3.28 allows different values for $r$ and $u$, in Figure 3.8, the number of observations, $r$ and $u$ are kept equal at each point, for ease of illustration. It can be seen that as $r$ and $u$ increase, the expected reliability $E[R(t)]$, tends to the value of reliability computed at point estimates $\hat{\lambda}$ and $\hat{\mu}$ (which are the true values for $\lambda$ and $\mu$, as $r$ and $u$ tend to $\infty)$.

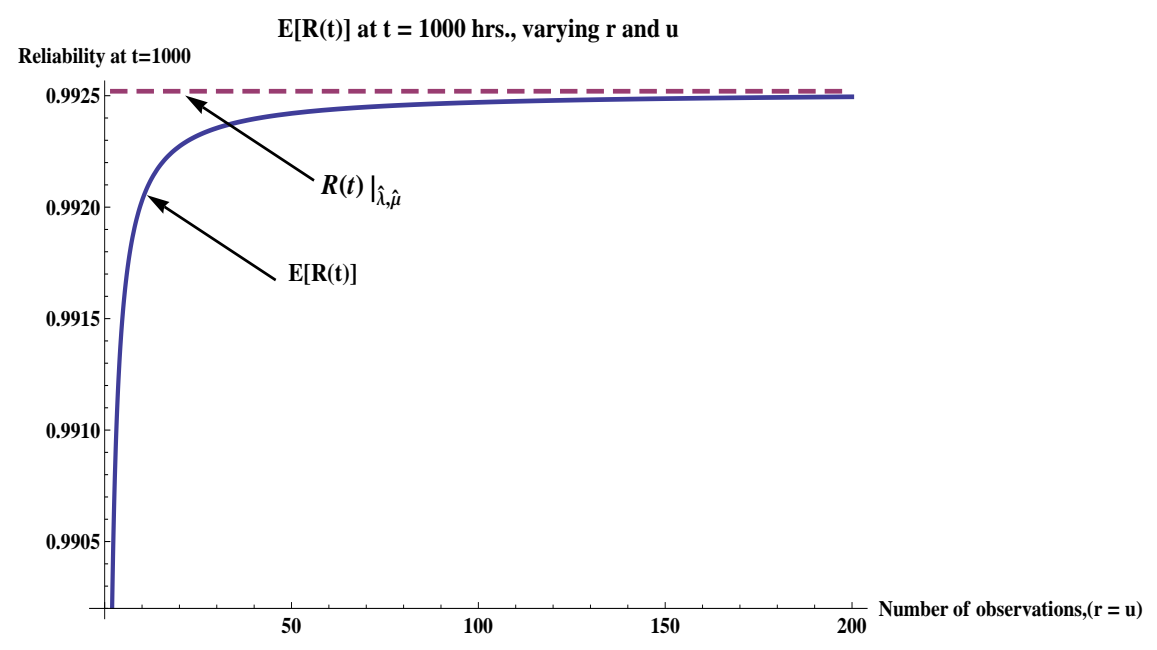

FigurE 3.8: Expected Reliability: Duplex System with one Spare

\subsubsection{Variance of reliability : Duplex System with a Spare}

For a duplex system with one spare, the variance of reliability, due to epistemic uncertainty in the rate parameters of times to failure distribution for components in active and standby mode, can be computed using the expected reliability $E[R(t)]$ and the second moment of reliability, $E\left[(R(t))^{2}\right]$. While $E[R(t)]$ has already been 
derived in Equation 3.28, $E\left[(R(t))^{2}\right]$ is derived below:

$$
\begin{aligned}
& E\left[(R(t))^{2}\right]=\left(\frac{s}{s+4 t}\right)^{r}\left(\frac{1}{s+4 t}\right)\left[\frac{4 b^{2} r(r+1)}{(u-1)(u-2)}\left(\frac{1}{s+4 t}\right)\right. \\
& -\frac{8 r(r+1) b^{2}}{(u-1)(u-2)}\left(\frac{b}{b+t}\right)^{u-2}\left(\frac{1}{s+4 t}\right) \\
& +\frac{4 r(r+1) b^{2}}{(u-1)(u-2)}\left(\frac{b}{b+2 t}\right)^{u-2}\left(\frac{1}{s+4 t}\right)+\frac{4 b \times r}{u-1} \\
& \left.-\frac{4 r \times b}{u-1}\left(\frac{b}{b+t}\right)^{u-1}\right]+\left(\frac{s}{s+4 t}\right)^{r} \\
& =\left(\frac{1}{1+4 \hat{\lambda} t / r}\right)^{r}\left(\frac{1}{r / \hat{\lambda}+4 t}\right)\left[\frac{4 u^{2} r(r+1)}{\hat{\mu}^{2}(u-2)}\left(\frac{1}{r / \hat{\lambda}+4 t}\right)\right. \\
& -\frac{8 r(r+1) u^{2}}{(u-1)(u-2) \hat{\mu}^{2}}\left(\frac{1}{1+\hat{\mu} t / u}\right)^{u-2}\left(\frac{1}{r / \hat{\lambda}+4 t}\right) \\
& +\frac{4 r(r+1) u^{2}}{\hat{\mu}^{2}(u-1)(u-2)}\left(\frac{1}{1+2 \hat{\mu} t / u}\right)^{u-2}\left(\frac{1}{r / \hat{\lambda}+4 t}\right)+\frac{4 r \times u}{\hat{\mu}(u-1)} \\
& \left.-\frac{4 r \times u}{\hat{\mu}(u-1)}\left(\frac{1}{1+\hat{\mu} t / u}\right)^{u-1}\right]+\left(\frac{1}{1+4 \hat{\lambda} t / r}\right)^{r}
\end{aligned}
$$

The variance $\operatorname{Var}[R(t)]$ can be derived next from Equations 3.28 and 3.29, simply using the relation $\operatorname{Var}[R(t)]=E\left[(R(t))^{2}\right]-(E[R(t)])^{2}$. It can also be shown that as the number of observations used to compute the estimates of parameters $\lambda$ and $\mu$ tend to $\infty$, the variance of reliability tends to 0 , as expected. Figure 3.9 shows the variance of reliability of this system (at $t=1000$ hours), as $r$ and $u$ are varied. It is clear that the variance tends to 0 as $r$ and $u$ increase. 


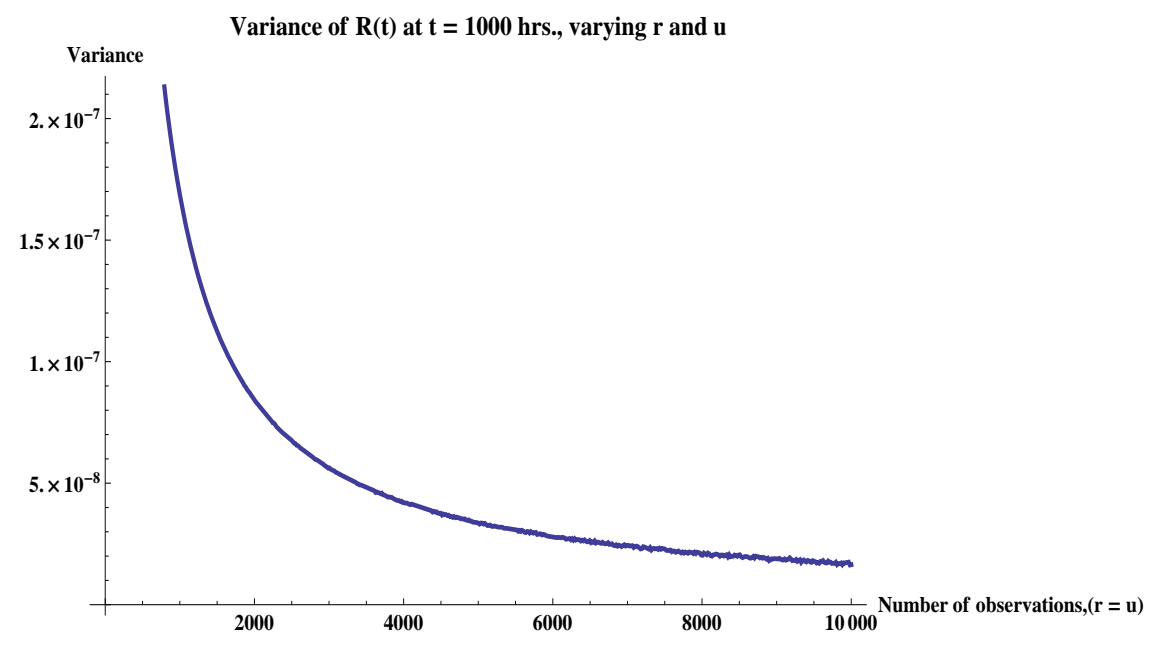

Figure 3.9: Variance of Reliability: Duplex System with one Spare

\subsection{Reliability of Recovery Block Architecture}

Consider a slightly more complex model of a recovery block architecture (RB) implemented with hot standby hardware [41]. The system is able to tolerate one hardware fault (due to one hot standby hardware server also running RB software) and one software fault. The reliability model considers imperfect coverage of hardware faults (coverage parameter) and different failure rates of different components (hardware and software). The Markov chain reliability model of the system is reproduced below in Figure 3.10. In Figure 3.10, states 1 and 2 are $U P$ states while states $S F$ and $U F$ (safe failure and unsafe failure state, respectively)are $D O W N$ states. For details of the reliability model, the reader is referred to [41]. The reliability of this system is given by:

$$
R_{R B}(t)=2 c e^{-\left(\lambda_{H}+\lambda_{S D}+\lambda_{S U}\right) t}-(2 c-1) e^{-\left(2 \lambda_{H}+\lambda_{S D}+\lambda_{S U}\right) t}
$$

where, $\lambda_{H}$ is the hardware component failure rate, $\lambda_{S D}$ and $\lambda_{S U}$ are the rates for detected and undetected failures and $c$ is probability of coverage for hardware failure.

Using the epistemic density functions for rate parameters as shown in Equation 3.4 and for the coverage parameter as shown in Equation 3.5, the expected reliability 


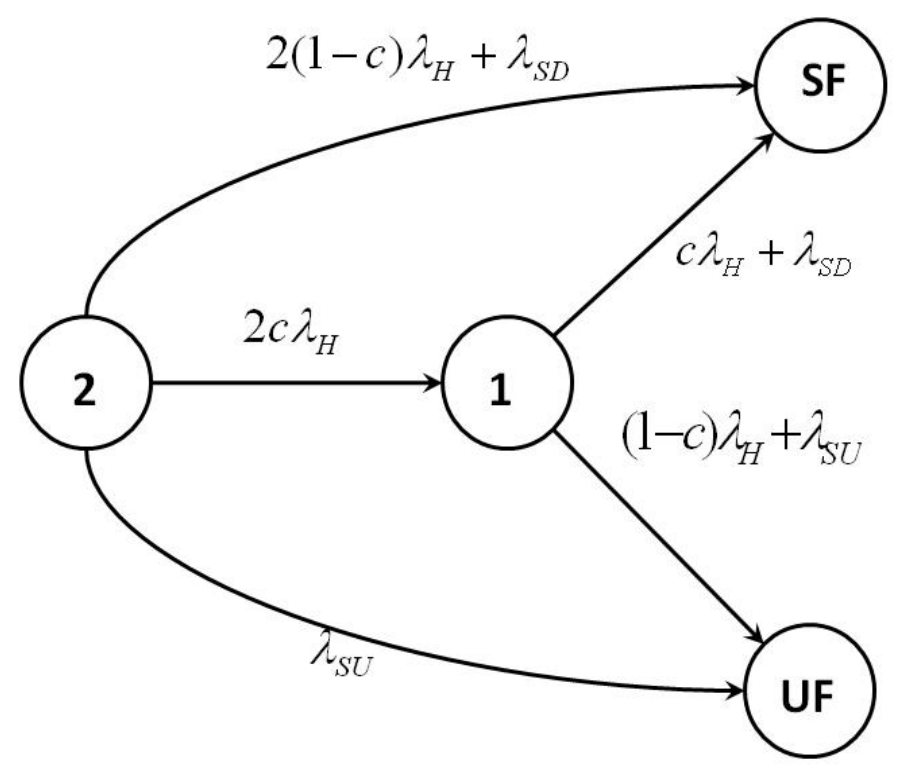

FiguRE 3.10: Reliability Model of a Recovery Block Architecture

of the $R B$ system, due to epistemic uncertainties in the parameters is obtained as:

$$
\begin{aligned}
E\left[R_{R B}(t)\right]= & \int_{0}^{\infty} \int_{0}^{\infty} \int_{0}^{1} R_{R B}(t) f_{\Lambda_{H}}\left(\lambda_{H}\right) f_{\Lambda_{S D}}\left(\lambda_{S D}\right) \\
& f_{\Lambda_{S U}}\left(\lambda_{S U}\right) f_{C}(c) d \lambda_{H} d \lambda_{S D} d \lambda_{S U} d c
\end{aligned}
$$

Evaluating the integral in Equation 3.31, the expected reliability of the recovery block architecture, at time $t$, can be obtained as:

$$
\begin{aligned}
E\left[R_{R B}(t)\right]= & \left(\frac{s_{1}}{s_{1}+t}\right)^{r_{1}}\left(\frac{s_{2}}{s_{2}+t}\right)^{r_{2}} \\
& \left(\left(\frac{s}{s+2 t}\right)^{r}\left(\frac{r_{i n j}-2 y}{r_{i n j}+2}\right)+2\left(\frac{s}{s+t}\right)^{r}\left(\frac{1+y}{r_{i n j}+2}\right)\right) \\
= & \left(\frac{1}{1+\hat{\lambda}_{S D} t / r_{1}}\right)^{r_{1}}\left(\frac{1}{1+\frac{\hat{\lambda}_{S U} t}{r_{2}}}\right)^{r_{2}} \\
& \left(\left(\frac{1}{1+\frac{2 \hat{\lambda}_{H} t}{r}}\right)^{r}\left(\frac{1-2 \hat{c}}{1+\frac{2}{r_{i n j}}}\right)+2\left(\frac{1}{1+\frac{\hat{\lambda}_{H} t}{r}}\right)^{r}\left(\frac{\hat{c}+1}{1+\frac{2}{r_{i n j}}}\right)\right)
\end{aligned}
$$


where, $r$ is the number of observed failures used to compute the point estimate $\hat{\lambda_{H}}$ and $s$ is the value of random variable $S$, denoting the sum of observed times to failure of the hardware. Similarly, $r_{1}$ and $r_{2}$ are the number of observations used to compute the point estimates $\hat{\lambda}_{S D}$ and $\hat{\lambda}_{S U}$, respectively and $s_{1}$ and $s_{2}$ are the values of random variable $S_{1}$ and $S_{2}$ denoting the sum of observed times to failure used to compute $\hat{\lambda}_{S D}$ and $\hat{\lambda}_{S U}$, respectively. $y$ is the value of number of successfully covered hardware faults random variable, $Y$, out of $r_{i n j}$ faults injected in the fault injection experiment to compute the point estimate of hardware fault coverage, $\hat{c}$.

Using the identity $\lim _{h \rightarrow \infty}(1+1 / h)^{h}=e$, it can be seen that as the number of observations/fault injections (i.e., $r_{1}, r_{2}$ and $r_{i n j}$ ) used to compute the point estimates of the model parameters increases (tends to $\infty$ ), the expected reliability of $R B, E\left[R_{R B}(t)\right]$, tends to $R(t)$ computed at the point estimates of the parameter values (which are the true values at infinite sample size).

Based on Equations 3.2 and 3.3, as shown in Sections 3.2 and 3.5, the variance of reliability of $R B$ architecture at time $t, \operatorname{Var}\left[R_{R B}(t)\right]$ due to epistemic uncertainties in the input parameters of the reliability model, is derived as:

$$
\begin{aligned}
\operatorname{Var}\left[R_{R B}(t)\right]= & 4\left(\frac{1}{6+5 r_{i n j}+r_{i n j}{ }^{2}}\right)\left(\frac{s_{1}}{s_{1}+2 t}\right)^{r_{1}}\left(\frac{s_{2}}{s_{2}+2 t}\right)^{r_{2}} \\
& \times\left[\left(\frac{s}{s+2 t}\right)^{r}(1+y)(2+y)\right. \\
& \left.+\left(\frac{s}{s+4 t}\right)^{r}\left(2+r_{i n j}+r_{i n j}{ }^{2}-4 r_{i n j} y+4 y^{2}\right)\right] \\
& \left.\left.-\left(\frac{1}{\left(2+r_{i n j}\right)^{2}}\right)\left(\frac{s_{1}}{s_{1}+t}\right)^{2 r_{1}}\left(\frac{s_{2}}{s_{2}+t}\right)^{2 r_{2}}\right)^{2 r}\left(r_{i n j}-2 y\right)^{2}+4\left(\frac{s}{s+t}\right)^{2 r}(1+y)^{2}\right) \\
& \left(\left(\frac{s}{s+2 t}\right)^{2 r}\right)
\end{aligned}
$$


Using the relation between point estimates, number of observations used to compute them and the values of sum of observed times to failure random variable for the rate parameters, as $s=r / \hat{\lambda}_{H}, s_{1}=r_{1} / \hat{\lambda}_{S D}$ and $s_{2}=r_{2} / \hat{\lambda}_{S U}$ as well as the relation $\hat{c}=y / r_{i n j}$, for the coverage probability of hardware fault, Equation 3.33 can be re-written as:

$$
\begin{aligned}
& \operatorname{Var}\left[R_{R B}(t)\right]=4\left(\frac{1}{\frac{6}{r_{\mathrm{inj}}{ }^{2}}+\frac{5}{r_{\mathrm{inj}}}+1}\right)\left(\frac{1}{1+\frac{2 \hat{\lambda}_{\mathrm{SD}} t}{r_{1}}}\right)^{r_{1}}\left(\frac{1}{1+\frac{2 \hat{\lambda}_{\mathrm{SU}} t}{r_{2}}}\right)^{r_{2}} \\
& \times\left[\left(\frac{1}{1+\frac{2 \hat{\lambda}_{H} t}{r}}\right)^{r}\left(\hat{c}+\frac{1}{r_{\mathrm{inj}}}\right)\left(\hat{c}+\frac{2}{r_{\mathrm{inj}}}\right)\right. \\
& \left.+\left(\frac{1}{1+\frac{4 \hat{\lambda}_{H} t}{r}}\right)^{r}\left(\frac{2}{r_{\mathrm{inj}}^{2}}+\frac{1}{r_{\mathrm{inj}}}+1-4 \hat{c}+4 \hat{c}^{2}\right)\right] \\
& -\left(\frac{1}{\left(\frac{2}{r_{\mathrm{inj}}}+1\right)^{2}}\right)\left(\frac{1}{1+\frac{\hat{\lambda}_{\mathrm{SD}} t}{r_{1}}}\right)^{2 r_{1}}\left(\frac{1}{1+\frac{\hat{\lambda}_{\mathrm{SU}} t}{r_{2}}}\right)^{2 r_{2}} \\
& \left(\left(\frac{1}{1+\frac{2 \hat{\lambda}_{H} t}{r}}\right)^{2 r}(1-2 \hat{c})^{2}+4\left(\frac{1}{1+\frac{\hat{\lambda}_{H} t}{r}}\right)^{2 r}(1+\hat{c})^{2}\right)
\end{aligned}
$$

Again, using the identity $\lim _{h \rightarrow \infty}(1+1 / h)^{h}=e$, it can be shown that as the number of observations used to compute point estimates of each of the parameters tend to $\infty$, the variance of reliability, due to epistemic uncertainties in the parameters, tends to 0 .

\subsection{Reliability of Composite Web Service}

We perform uncertainty propagation through the service reliability and performance model of a composite web service called Travel-Agent which was discussed in [75]. 
A composite web service combines several different web services to provide a new service to its users. It uses high level languages such as BPEL (Business Process Execution Language) [3] to quickly combine the existing web services into new services. The web service Travel-Agent, interacts with different services to make airlines and hotel reservations for customers. Each of the individual interactions (web services) may have complex interactions within themselves and have their own mean response time and reliability. When initialized, it first looks up for vacancy, fare and other information from two different airlines, in parallel. Upon getting the response from both, it chooses one of them, based on some requirements (e.g., fare, schedule, etc.). If only one of them responds, it chooses the only response. Then it continues to make airlines reservation for the chosen airlines. After making airline reservation, it makes hotel reservation and notifies the user. Each of these operations may fail and can be retried/restarted. The entire process is considered successful only if a successful airline and hotel reservation is made and a reply is sent back to the user. The look up for vacancy in airlines happens concurrently on both airlines. While the details of the service can be obtained from [75], the BPEL representation of the service, has been reproduced in Figure 5.12, for ease of reference. A CTMC model is formulated to study the overall reliability and response time of the entire composite web service. The response time of each of these services is assumed to follow an exponential distribution. The reliability of each of the individual services is computed as the ratio of number of successful executions of the service to the total number of executions of the service attempted and so is the parameter of Bernoulli aleatory distribution. The CTMC considers concurrent execution of the two airlines services, failure of any of the services and restarts/retries of services to attempt recovery from failure of a service. It also considers overhead of time spent in restarts (assuming this overhead time to follow an exponential distribution) as well as imperfect coverage of restarts of services. While the details of the CTMC model can be seen at [75], it has been 


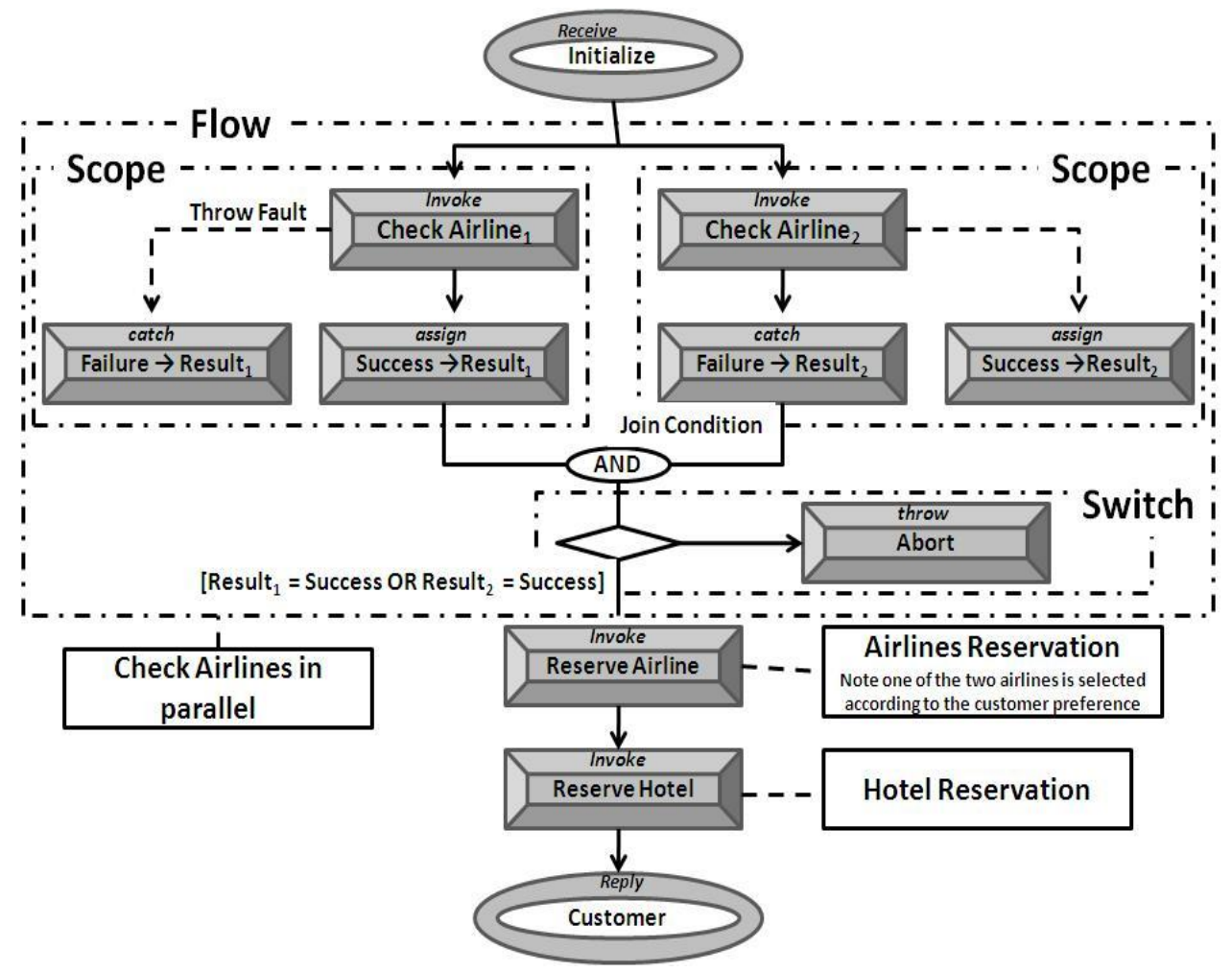

Figure 3.11: BPEL representation of Travel-Agent Process

reproduced in Figure 5.13, for ease of reference. In the CTMC in Figure 5.13, the "Initialization" has mean response time of $m r s p_{i}$ and reliability $R_{i}$. Upon failure of this state, it is restarted with mean overhead time $m r_{i}$ (from state RIni). The restart can be successful with a coverage parameter $C_{i}$. The concurrent airline selection process has been broken down into 5 states. " $A_{1} \mid A_{2}$ " depicts the state when both airlines selection processes are ongoing. Rest of the four states depict one of the airlines selection process ongoing while the other has either finished or failed. Retry is not considered for airlines selection process. The mean response times for airline selection process are $m r s p_{a 1}$ and $m r s p_{a 2}$ and reliabilities are $R_{a 1}$ and $R_{a 2}$, for airlines 1 and 2, respectively. Restart and imperfect coverage of restart is considered for the states airline reservation "A resv", hotel reservation "HT resv" and "Reply to customer". 


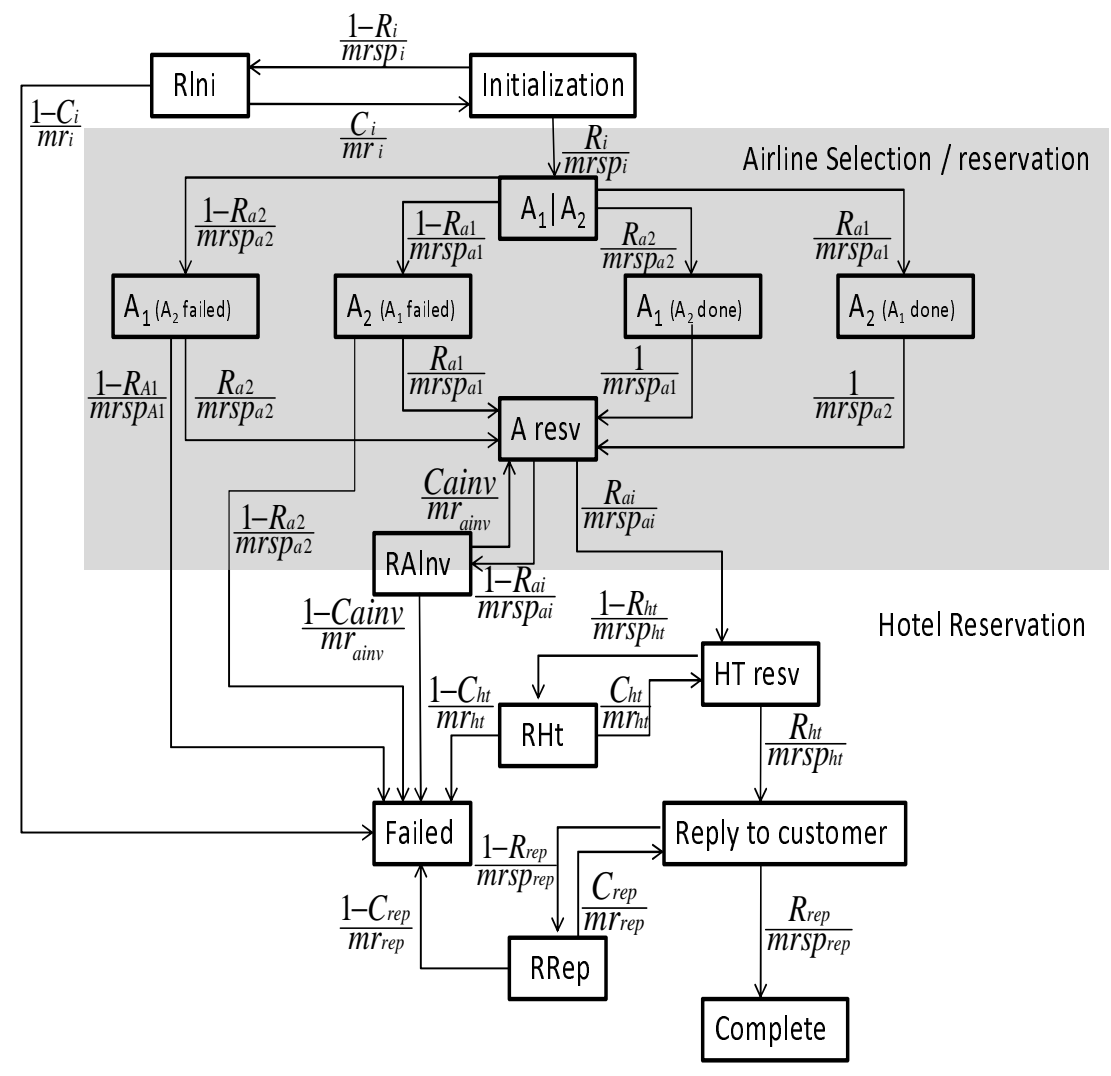

Figure 3.12: CTMC Model of Composite Web Service

A closed-form expression for reliability of the composite web service was derived in $[75]$ as:

$$
\begin{aligned}
R_{s y s}= & \underbrace{\left(\frac{R_{i}}{1-C_{i}\left(1-R_{i}\right)}\right)}_{(i)} \underbrace{\left(\frac{\left(R_{a 1}+R_{a 2}-R_{a 1} \times R_{a 2}\right) R_{a i}}{1-C_{a i}\left(1-R_{a i}\right)}\right)}_{(i i)} \\
& \underbrace{\left(\frac{R_{h t}}{1-C_{h t}\left(1-R_{h t}\right)}\right)}_{(i i i)} \underbrace{\left(\frac{R_{r e p}}{1-C_{r e p}\left(1-R_{r e p}\right)}\right)}_{(i v)}
\end{aligned}
$$

While the expression for reliability of the composite web service is difficult to analytically integrate (several variables and complex expression of reliability), the expressions for lower and upper bounds of reliability, obtained by assuming no coverage 
(coverage probabilities $=0$ ) and perfect coverage, respectively are simpler expressions, where closed form propagation of epistemic uncertainty can be performed and the expected reliability as well as the variance of reliability thus obtained can be analyzed. The expression for lower bound of reliability (obtained by assuming $C_{i}=1$ and all other coverage probabilities $=0$ ) of the composite web service is reproduced:

$$
R_{\text {sys-lb }}=\left(R_{a 1}+R_{a 2}-R_{a 1} \times R_{a 2}\right) \times R_{a i} \times R_{h t} \times R_{r e p}
$$

In the reliability model of the composite web service, since the input parameters of the model are all parameters of Bernoulli aleatory distribution (probability of successful completion of a service and coverage probabilities), the epistemic distribution of each of the parameters is a beta distribution, as derived in Equation 3.5. Therefore using the epistemic distribution as derived in Equation 3.5, based on Equation 3.2 the expected reliability lower bound of the composite web service is derived as:

$$
\begin{aligned}
E\left[R_{\text {sys-lb }}\right]= & \frac{\left(\frac{1}{r_{1}}+\frac{1}{r_{2}}+\frac{3}{r_{1} r_{2}}+\hat{R}_{a 1}+\frac{\hat{R}_{a 1}}{r_{2}}+\hat{R}_{a 2}+\frac{\hat{R}_{a 2}}{r_{1}}-\hat{R}_{a 1} \hat{R}_{a 2}\right)}{\left(1+\frac{2}{r_{1}}\right)\left(1+\frac{2}{r_{2}}\right)} \\
& \times \frac{\left(\frac{1}{r_{3}}+\hat{R}_{a i}\right)\left(\frac{1}{r_{4}}+\hat{R}_{h t}\right)\left(\frac{1}{r_{5}}+\hat{R}_{r e p}\right)}{\left(1+\frac{2}{r_{3}}\right)\left(1+\frac{2}{r_{4}}\right)\left(1+\frac{2}{r_{5}}\right)}
\end{aligned}
$$

Using simple algebraic manipulations, it can be shown that as the number of attempted executions $\left(r_{1}, r_{2}, r_{3}, r_{4}\right.$ and $\left.r_{5}\right)$ of the individual services to compute the point estimates $\left(\hat{R}_{a 1}, \hat{R}_{a 2}, \hat{R}_{a i}, \hat{R}_{h t}\right.$ and $\hat{R}_{r e p}$, respectively), tend to $\infty$, the expected value of lower bound of the overall reliability of the composite web service tends to the reliability $R_{s y s-l b}$ computed at point estimates of the parameters.

Similarly, based on Equations and 3.2 and 3.3, the variance of the lower bound of reliability of composite web service, due to epistemic uncertainties in the input 
parameters of the reliability model, is obtained as:

$$
\begin{aligned}
\operatorname{Var}\left[R_{\text {sys }-l b]}\right]= & {\left[\frac{\left(\hat{R}_{\mathrm{a} 1}+\frac{1}{r_{1}}\right)\left(\hat{R}_{\mathrm{a} 1}+\frac{2}{r_{1}}\right)\left(\frac{1}{r_{1}}+1-\hat{R}_{\mathrm{a} 2}\right)\left(\frac{2}{r_{1}}+1-\hat{R}_{\mathrm{a} 2}\right)}{\left(1+\frac{5}{r_{1}}+\frac{6}{r_{1}{ }^{2}}\right)\left(1+\frac{5}{r_{2}}+\frac{6}{r_{2}{ }^{2}}\right)}\right.} \\
& +\frac{2\left(\hat{R}_{\mathrm{a} 1}+\frac{1}{r_{1}}\right)\left(\hat{R}_{\mathrm{a} 2}+\frac{1}{r_{2}}\right)\left(\frac{1}{r_{2}}+1-\hat{R}_{\mathrm{a} 2}\right)}{\left(1+\frac{2}{r_{1}}\right)\left(1+\frac{5}{r_{2}}+\frac{6}{r_{2}{ }^{2}}\right)} \\
& \left.+\frac{\left(\hat{R}_{\mathrm{a} 2}+\frac{1}{r_{2}}\right)\left(\hat{R}_{\mathrm{a} 2}+\frac{2}{r_{2}}\right)}{\left(1+\frac{5}{r_{2}}+\frac{6}{r_{2}{ }^{2}}\right)}\right] \\
& \frac{\left(\hat{R}_{\mathrm{ai}}+\frac{1}{r_{3}}\right)\left(\hat{R}_{\mathrm{ai}}+\frac{2}{r_{3}}\right)\left(\hat{R}_{\mathrm{ht}}+\frac{1}{r_{4}}\right)\left(\hat{R}_{\mathrm{ht}}+\frac{2}{r_{4}}\right)}{\left(1+\frac{5}{r_{3}}+\frac{6}{r_{3}{ }^{2}}\right)\left(1+\frac{2}{r_{4}}\right)\left(1+\frac{3}{r_{4}}\right)} \\
& \times \frac{\left(\hat{R}_{\mathrm{rep}}+\frac{1}{r_{5}}\right)\left(\hat{R}_{\mathrm{rep}}+\frac{2}{r_{5}}\right)}{\left(1+\frac{2}{r_{5}}\right)\left(1+\frac{3}{r_{5}}\right)}-E\left[R_{s y s-l b}\right]^{2}
\end{aligned}
$$

Using simple algebraic manipulations, it can be shown that as the number of attempted executions of the individual services tend to $\infty$, the variance of lower bound of the overall reliability of the composite web service, due to the epistemic uncertainties in the parameter values, tends to 0 .

\subsubsection{Comparison of Variance and Expectation of Reliability}

We compare the expectation and variance of the reliability of the composite web service computed by the closed-form analytic integration method discussed in this chapter to that obtained by the method of performing manipulations on the Taylor series expansion of the expression of reliability, discussed by Yin and Trivedi in [93]. Based on the method in [93], using Equation 1.8, the expected value of lower bound of reliability of the composite web service $R_{s y s-l b}$ (Equation 3.36), is given by:

$$
E\left[R_{s y s-l b}\right] \approx\left(R_{a 1}+R_{a 2}-R_{a 1} \times R_{a 2}\right) \times R_{a i} \times R_{h t} \times\left. R_{r e p}\right|_{\mu_{R_{a 1}}, \mu_{R_{a 2}}, \mu_{R_{a i}}, \mu_{R_{h t}}, \mu_{R_{r e p}}}
$$


where $\mu_{Y}$ is the mean of the input parameter as random variable $Y$. In other words the expression for reliability evaluated at the mean of the parameters. As it can be seen here, the expression for expected value of $R_{s y s-l b}$ is different from the one obtained by the method explained in this chapter in Equation 3.37. While the expected reliability computed by the method described by Yin and Trivedi in [93] in Equation 3.39 is approximate, the method in this chapter (Equation 3.37) provides the exact value for $E\left[R_{\text {sys-lb }}\right]$. Equation 3.39 requires the mean of the aleatory model input parameters to compute the expected value of the aleatory model output, whereas Equation 3.37 takes the point estimate of input parameters and the number of observations used to compute the point estimate, as its input.

Similarly, based on Equation 1.9, the variance of the lower bound of reliability of the composite web service $R_{\text {sys-lb }}$, due to the epistemic uncertainties in the model input parameters, is given by:

$$
\begin{aligned}
& \operatorname{Var}\left[R_{\text {sys-lb }}\right] \approx\left(1-R_{\mathrm{a} 2}\right)^{2} R_{\mathrm{ai}}^{2} R_{\mathrm{ht}}^{2} R_{\mathrm{rep}}^{2} \operatorname{var}_{R_{\mathrm{a} 1}}+\left(1-R_{\mathrm{a} 1}\right)^{2} R_{\mathrm{ai}}^{2} R_{\mathrm{ht}}^{2} R_{\mathrm{rep}}^{2} \operatorname{var}_{R_{\mathrm{a} 2}}+ \\
& R_{\mathrm{ai}}^{2} R_{\mathrm{ht}}^{2} R_{\mathrm{rep}}^{2} \operatorname{var}_{R_{\mathrm{a} 1}} \operatorname{var}_{R_{\mathrm{a} 2}}+\left(R_{\mathrm{a} 1}+R_{\mathrm{a} 2}-R_{\mathrm{a} 1} R_{\mathrm{a} 2}\right)^{2} R_{\mathrm{ht}}^{2} R_{\mathrm{rep}}^{2} \operatorname{var}_{R_{\mathrm{a} i}}+ \\
& \left(1-R_{\mathrm{a} 2}\right)^{2} R_{\mathrm{ht}}^{2} R_{\mathrm{rep}}^{2} \operatorname{var}_{R_{\mathrm{a} 1}} \operatorname{var}_{R_{\mathrm{ai}}}+\left(1-R_{\mathrm{a} 1}\right)^{2} R_{\mathrm{ht}}^{2} R_{\mathrm{rep}}^{2} \operatorname{var}_{R_{\mathrm{a} 2}} \operatorname{var}_{R_{\mathrm{ai}}}+ \\
& \left(R_{\mathrm{a} 1}+R_{\mathrm{a} 2}-R_{\mathrm{a} 1} R_{\mathrm{a} 2}\right)^{2} R_{\mathrm{ai}}^{2} R_{\mathrm{rep}}^{2} \operatorname{var}_{R_{\mathrm{ht}}}+\left(1-R_{\mathrm{a} 2}\right)^{2} R_{\mathrm{ai}}^{2} R_{\mathrm{rep}}^{2} \operatorname{var}_{R_{\mathrm{a} 1}} \operatorname{var}_{R_{\mathrm{ht}}}+ \\
& \left(1-R_{\mathrm{a} 1}\right)^{2} R_{\mathrm{ai}}^{2} R_{\mathrm{rep}}^{2} \operatorname{var}_{R_{\mathrm{a} 2}} \operatorname{var}_{R_{\mathrm{ht}}}+ \\
& \left(R_{\mathrm{a} 1}+R_{\mathrm{a} 2}-R_{\mathrm{a} 1} R_{\mathrm{a} 2}\right)^{2} R_{\mathrm{rep}}^{2} \operatorname{var}_{R_{\mathrm{ai}}} \operatorname{var}_{R_{\mathrm{ht}}}+\left(R_{\mathrm{a} 1}+R_{\mathrm{a} 2}-R_{\mathrm{a} 1} R_{\mathrm{a} 2}\right)^{2} R_{\mathrm{ai}}^{2} R_{\mathrm{ht}}^{2} \operatorname{var}_{R_{\mathrm{rep}}}+ \\
& \left(1-R_{\mathrm{a} 2}\right)^{2} R_{\mathrm{ai}}^{2} R_{\mathrm{ht}}^{2} \operatorname{var}_{R_{\mathrm{a} 1}} \operatorname{var}_{R_{\mathrm{rep}}}+\left(1-R_{\mathrm{a} 1}\right)^{2} R_{\mathrm{ai}}^{2} R_{\mathrm{ht}}^{2} \operatorname{var}_{R_{\mathrm{a} 2}} \operatorname{var}_{R_{\mathrm{rep}}}+ \\
& \left(R_{\mathrm{a} 1}+R_{\mathrm{a} 2}-R_{\mathrm{a} 1} R_{\mathrm{a} 2}\right)^{2} R_{\mathrm{ht}}^{2} \operatorname{var}_{R_{\mathrm{ai}}} \operatorname{var}_{R_{\mathrm{rep}}}+ \\
& \left.\left(R_{\mathrm{a} 1}+R_{\mathrm{a} 2}-R_{\mathrm{a} 1} R_{\mathrm{a} 2}\right)^{2} R_{\mathrm{ai}}^{2} \operatorname{var}_{R_{\mathrm{ht}}} \operatorname{var}_{R_{\mathrm{rep}}}\right|_{\mu_{R_{a 1}, \mu_{R_{a 2}}, \mu_{R_{a i}}, \mu_{R_{h t}}, \mu_{R_{r e p}}}}
\end{aligned}
$$

The variance of the lower bound of reliability of the composite web service $R_{\text {sys-lb }}$ computed as in Equation 3.40, provides an approximate value of reliability. However, Equation 3.38 provides an expression for the exact value of variance of the 
lower bound of reliability of the composite web service. Equation 3.40 (based on the method used by Yin and Trivedi [93]) requires the variance and mean of each of the model input parameters as its input, whereas the method explained in this chapter (resulting in Equation 3.38) does not require computation of variance and mean of each of the input parameter, but uses the point estimates of the aleatory model input parameters and the number of observations used to compute them. The method of manipulating the Taylor series expansion of the model output provides only the approximate expressions for expected value and variance of the model output, whereas the method discussed in this chapter provides the exact expression for the expected value and variance as well as the distribution of the aleatory model output, due to the epistemic uncertainties in the aleatory model input parameters.

\subsection{Interval Availability: Cumulative Downtime Distribution Method}

Fricks et. al. [21] propose a method for computing interval availability of systems, from failure data. In this method, the interval availability of system, $A_{I}(T)$, over the time interval $T$ is obtained by:

$$
A_{I}(T)=1-\frac{\sum_{i=1}^{n} d_{i}}{n \times T}
$$

where, $d_{i}$ s are the values of $n$ independent and identically distributed random variables $D_{i} \mathrm{~s}$, following exponential distribution (aleatory) with parameter $\mu$ and denoting the $n$ down times of the system observed during the period $T$. In this method of interval availability calculation, there is only one epistemic random variable, $M$. Using $S=\sum_{i=1}^{n} D_{i}$, the total observed down time random variable $S$ with value $s$, and $n / S=M$, Equation 3.41 can be written as:

$$
A_{I}(T)=1-\frac{1}{\mu T}
$$


Since the down time of the system is assumed to follow an exponential aleatory distribution, then as shown in Equation 3.4, epistemic $p d f$ of $\mu$ will be $n$-stage Erlang with parameter $s$. Performing the unconditioning integrals as explained in Section 3.1, using this epistemic distribution, the distribution, expectation and variance of interval availability computed by the cumulative downtime distribution (CDD) method, due to the epistemic uncertainty in repair rate, have been derived next.

\subsubsection{Distribution of Interval Availability}

The distribution of interval availability of a system calculated by CDD method,due to the epistemic uncertainty in parameter $\mu$ can be derived using Equations 3.1 and 3.4, as shown in Section 3.2.1. Equation 3.15 gives the CDF of reliability of a TMR system.

$$
F_{A_{I}(T)}(a)=\int_{0}^{\infty} I\left(A_{I}(T) \leq a\right) \frac{\mu^{n-1} s^{r} e^{-\mu s}}{(n-1) !} d \mu
$$

The above integral is non-zero only for values of $\mu$ for which $A_{I}(T) \leq a$. Since $A_{I}(T)=1-1 /(\mu T)$, it can be shown that the integral will be non-zero for $\mu \leq \mu_{a}$, such that $\mu_{a}=1 /(T(1-a))$. Using the CDF of an Erlang distributed random variable, Equation 3.43 then reduces to:

$$
\begin{aligned}
F_{A_{I}(T)}(a) & =\int_{0}^{\infty} I\left(A_{I}(T) \leq a\right) \frac{\mu^{n-1} s^{r} e^{-\mu s}}{(n-1) !} d \mu \\
& =\underbrace{\int_{0}^{\mu_{a}} \frac{\mu^{n-1} s^{n} e^{-\mu s}}{(n-1) !} d \mu}_{\text {Erlang } C D F} \\
& =1-\sum_{i=0}^{n-1} e^{-s \mu_{a}} \frac{\left(s \mu_{a}\right)^{i}}{i !}
\end{aligned}
$$

As discussed in Section 3.2.1, as the number of observations of down time, $n$, 
tends to $\infty, \hat{\mu}$ tends to the true value of $\mu$ and hence the distribution of $M$ is a Heaviside step function (Erlang distribution with infinite stages) with step at $\mu=$ $\hat{\mu}$. It follows that the CDF of interval availability of a system calculated by CDD method, $F_{A_{I}(T)}(a)$, as $n \rightarrow \infty$, tends to a Heaviside step function, $H[a-(1-1 /(\hat{\mu} T))]$, with the step at $1-1 /(\hat{\mu} T)$. Figure 3.13 shows the CDF of interval availability of a system calculated by CDD method, $A_{I}(T)$, as the number of observed downtimes $n$, is varied from 10 to 500 . It can be seen clearly that as $n$ increases, the CDF tends to the step function. The value of $\hat{\mu}$ is chosen to be $0.01 \mathrm{per} h r$. and interval availability is computed over $T=1000$ hrs..

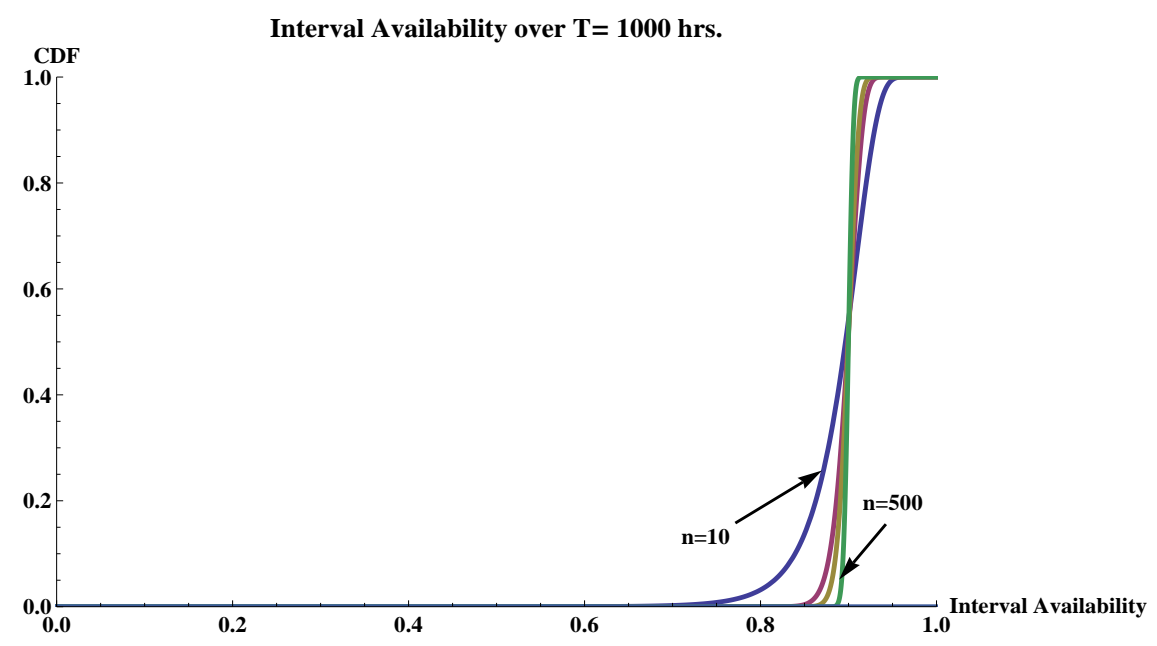

FIguRE 3.13: CDF of Interval Availability by CDD Method

\subsubsection{Expected Interval Availability}

Based on Equation 3.2, the unconditional expected interval availability of a system computed by the CDD method, for the interval $T$, can be computed as:

$$
\begin{aligned}
E\left[A_{I}(T)\right] & =\int_{0}^{\infty}\left(1-\frac{1}{\mu T}\right) \cdot \underbrace{\frac{\mu^{n-1} s^{n} e^{-\mu s}}{(n-1) !}}_{\text {Erlang pdf }} d \mu \\
& =1-\frac{s}{T(n-1)}=1-\frac{1}{T \hat{\mu}(1-1 / n)}
\end{aligned}
$$


As the number of downtimes observed in the period $T$ tends to $\infty$, the expected interval availability tends to $1-1 /(\hat{\mu} T)$, which is the true value.

Figure 3.14 plots the expected value of $A_{I}(T)$ over $T=1000 \mathrm{hrs}$., as a function of $n$. The value of $\hat{\mu}$ is chosen to be 0.01 per $h r$., as earlier. It can be seen that $E\left[A_{I}(T)\right]$ tends to $1-1 /(\hat{\mu} T)$, as $n$ increases.

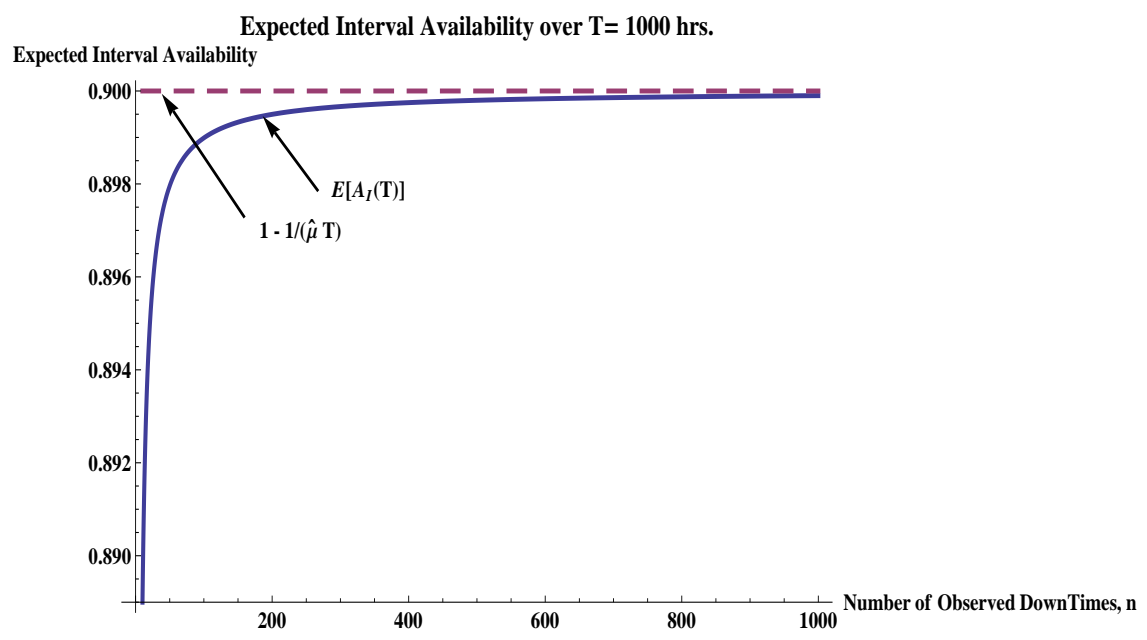

Figure 3.14: Expected Interval Availability

\subsubsection{Variance of Interval Availability}

Based on the Equations 3.3 and 3.45 and using the relation $\operatorname{Var}[Y]=E\left[Y^{2}\right]-$ $(E[Y])^{2}$, where $Y$ is a random variable, variance of interval availability of a system 
over a period $T$, due to the epistemic uncertainty in the repair rate $\mu$, is obtained as:

$$
\begin{aligned}
\operatorname{Var}\left[A_{I}(T)\right]= & \underbrace{\int_{0}^{\infty}\left(1-\frac{1}{\mu T}\right)^{2} \cdot \underbrace{\frac{\mu^{n-1} s^{n} e^{-\mu s}}{(n-1) !}}_{\text {Erlang } p d f}}_{E\left[\left(A_{I}(T)\right)^{2}\right]} \mu \\
& -\underbrace{\left(1-\frac{1}{T \hat{\mu}(1-1 / n)}\right)^{2}}_{\left(E\left[A_{I}(T)\right]\right)^{2}} \\
= & \frac{s^{2}}{T^{2}(n-1)^{2}(n-2)}=\frac{1}{T^{2}\left(1-\frac{1}{n}\right)^{2}(n-2)}
\end{aligned}
$$

It follows from Equation 3.47 that $\operatorname{Var}\left[A_{I}(T)\right] \rightarrow 0$ as $n \rightarrow+\infty$. 
4

\section{Numerical Integration Approach for Uncertainty Propagation}

As the expression for the output of the aleatory model gets more complex, analytical integration of the closed-form expression of aleatory model output, as shown is Equations 3.1 through 3.3, to propagate the epistemic uncertainty in input parameters of the aleatory model, may not always be possible. Integrating the expression may become intractable due to its complexity or number of variables (number of dimensions), or it may not be possible to express the integration in finite terms of familiar elementary functions (it may be difficult or impossible to find an antiderivative which is an elementary function). In such cases, numerical integration techniques may be used to perform the unconditioning integrations in Equations 3.1 through 3.3. In many cases, performing a numerical integration (though approximate), may be easier and faster than an analytic one. Unconditioning the model output via numerical integration may also be performed when closed-form solutions of model output do not exist. As demonstrated in examples towards the end of Chapter 3, analytic integration of expressions of aleatory model output becomes intractable for even simple 
expressions of model outputs. In this chapter we perform epistemic uncertainty propagation through numerical integration of closed-form expressions for aleatory model output of some example systems, with different output metrics (reliability, attack probability and average number of jobs in the system). Epistemic independence of the input parameters of the aleatory is assumed and the models considered in this chapter have output expressions more complex than those in Chapter $3^{1}$.

\subsection{Reliability of a Composite Web Service}

In Chapter 3, analytical closed-form uncertainty propagation was performed on the expression for the lower bound of reliability of a composite web service (discussed in [75]) and a brief overview of the example was provided. The reliability of a composite web service as shown in Equation 3.35 has a complex expression for which the unconditioning integrations become intractable due to the large number of variables (dimensions of integration), computational complexity and an expression not amenable to analytic integration. The expression for reliability of the composite web service is reproduced below for ease of reference in this chapter:

$$
\begin{aligned}
R_{\text {sys }}= & \underbrace{\left(\frac{R_{i}}{1-C_{i}\left(1-R_{i}\right)}\right)}_{(i)} \underbrace{\left(\frac{\left(R_{a 1}+R_{a 2}-R_{a 1} \times R_{a 2}\right) R_{a i}}{1-C_{a i}\left(1-R_{a i}\right)}\right)}_{(i i)} \\
& \underbrace{\left(\frac{R_{h t}}{1-C_{h t}\left(1-R_{h t}\right)}\right)}_{(i i i)} \underbrace{\left(\frac{R_{r e p}}{1-C_{r e p}\left(1-R_{r e p}\right)}\right)}_{(i v)}
\end{aligned}
$$

In this chapter we use the full expression for reliability of the composite web service as shown in Equation 4.1(and not just the simplified expression for the lower or upper bounds of it) to perform epistemic uncertainty propagation by analytic-numerical method. Assumption of epistemic independence between parameters allows for nu-

\footnotetext{
${ }^{1}$ Adapted from [53]
} 
merical integration of the independent factors $((i),(i i),(i i i)$ and $(i v)$ in Equation 4.1) of the expression for reliability, thus simplifying the unconditioning integrations. The coverage probabilities in the model, $C_{i}, C_{a i}, C_{h t}$ and $C_{r e p}$ are parameters of a Bernoulli aleatory distribution. Since the reliabilities of each of the individual web service $R_{i}, R_{a i}, R_{a 1}, R_{a 2}$ and $R_{h t}$ are computed in [75] as the ratio of successful invocations of the web service to the total number of invocations of that web service, they too are parameters of Bernoulli aleatory distribution. As explained in Section 2.3.3, the form of epistemic distribution of these reliability and coverage parameters is beta.

Using a beta epistemic distribution as derived in Equation 2.14, for each of the reliability and coverage parameters in Equation 4.1, based on Equation 3.2, the expected reliability of the composite web service is computed using the Global Adaptive method of numerical integration (a built-in method, supported by NIntegrate function in Mathematica [2]). The independent factors $((i),(i i),(i i i)$ and $(i v)$ in Equation 3.35) are numerically integrated using the Global Adaptive method, using the point estimates of reliability as well as coverage parameters, provided in [75]. Figure 4.1 shows the expected reliability of the composite web service. It can be seen that as the number of invocations of each individual service (to compute the reliability of each of the services) is increased, the expected reliability of the composite web service tends to the point estimate of reliability, $\hat{R}_{\text {sys }}$ (which tends to the true value of reliability as the number of invocations of each individual service tends to $\infty)$. In Figure 4.1, we have assumed the number of invocations of each service to be the same, for ease of illustration (the method however, allows for different number of invocations used to compute the reliability of different services).

Similarly, based on Equations 3.2 and 3.3, the variance of reliability of the composite web service can be obtained. The variance thus computed will tend to 0 as the number of invocations of each service tends to $\infty$ (indicating $\hat{R_{\text {sys }}}$ approaching 


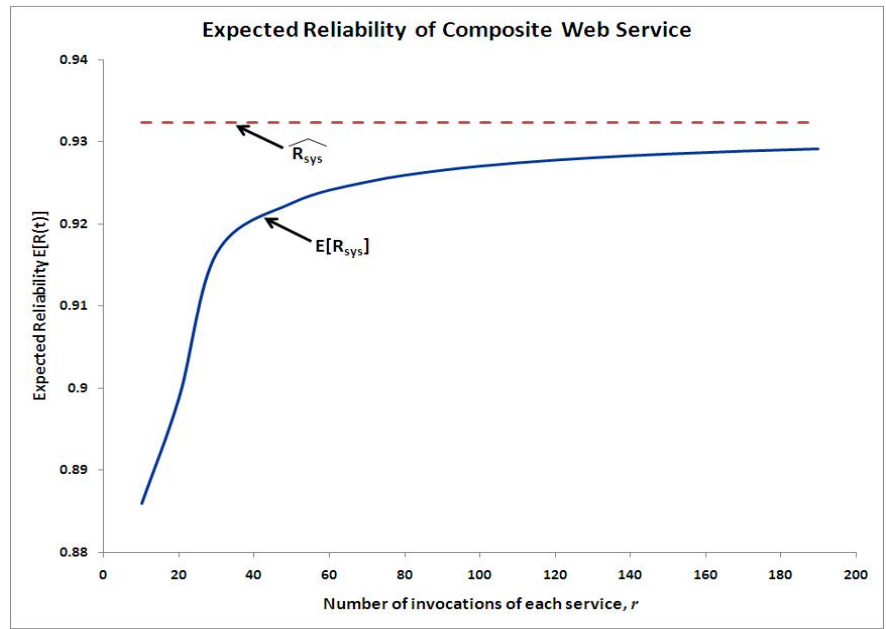

FiguRE 4.1: Expected Reliability of Composite Web Service

the true value).

\subsection{M/M/1 Queue with Server Breakdown and Repair}

We use the $M / M / 1$ queuing system with server breakdown and repair, explained in [7], as another example to illustrate analytic-numeric epistemic uncertainty propagation. In [7], an approximate expression for the average number of jobs in the system, is derived for an $M / M / 1$ system with breakdown and repair of the server, using an aggregation technique developed to handle the stiffness in the model due to orders of magnitude of difference in parameter values (rate of job arrival and service being much faster than the rate of server breakdown and repair). The Markov chain used to model the system in [7] has been reproduced in Figure 4.2, for ease of reference (while the figure in [7] is for an $M / M / 1 / m$ system with server breakdown and repair, here, we have modified the figure by setting $m$ to $\infty$, for it to model an $M / M / 1$ system with server breakdown and repair). The states of the Markov chain are denoted by $(l, k)$, where $l$ is the number of customers/jobs in the system and $k$ is the number of non-failed servers. $\lambda$ and $\mu$ are the customer arrival and service rates, 
respectively, while $\gamma$ and $\tau$ are the server failure and repair rates, respectively.

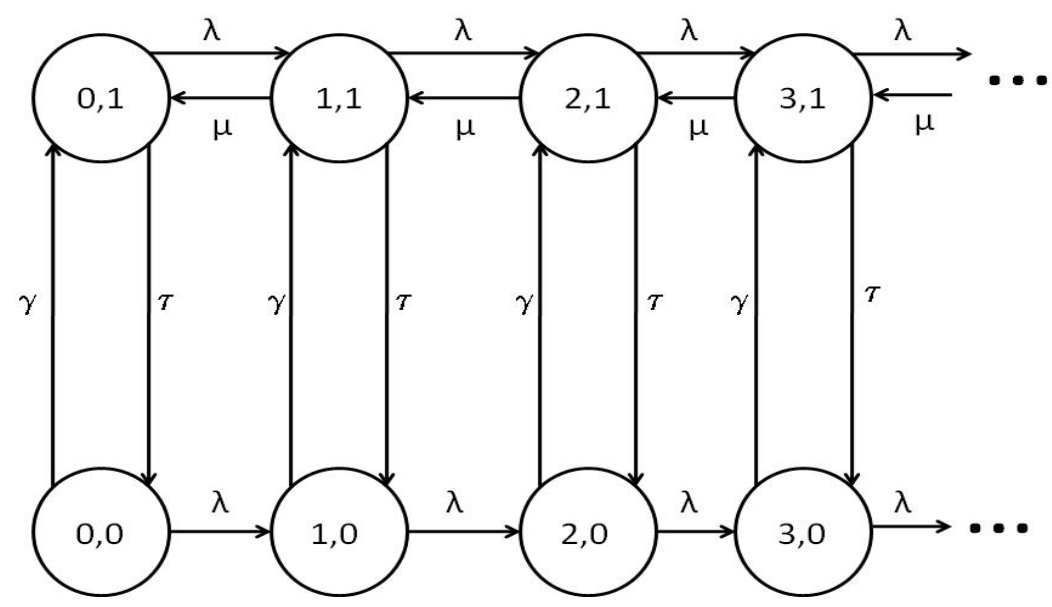

Figure 4.2: CTMC model of M/M/1 system with server breakdown and repair

An approximate expression for expected number of customers in the system, is derived in [7] as:

$$
\bar{N}=\frac{\rho}{1-\rho}+\frac{\lambda \gamma}{\tau(\gamma+\tau)(1-\rho)}
$$

where, $\rho=\lambda / \mu, \lambda$ and $\mu$ are the customer arrival and service rates, respectively, while $\gamma$ and $\tau$ are the server failure and repair rates, respectively.

Performing the unconditioning integrations (as shown in Equations 3.1 through 3.3) analytically is difficult, as the expression for $\bar{N}$ involves ratios of random variables with gamma distributions and a closed-form antiderivative for it is hard to obtain. Therefore, the unconditioning integrations have to be performed numerically.

Since the parameters in Equation 4.2, are all rate parameters of exponential aleatory distribution, the epistemic density function of each parameter is beta and its parameters can be derived as shown in Equation 3.4. The expected value and variance due to the epistemic uncertainties in the model input parameters, for the 
expected number of customers in the system are obtained by computing the integrations shown in Equations 3.2 and 3.3, numerically using the Global Adaptive method of numerical integration (a built-in method, supported by NIntegrate function in Mathematica [2]). To simplify the computations, we exploit the linearity property of expectation property of expectation of product of independent random variables. Figure 4.3 shows the expectation of $\bar{N}$, as the number of observations used to compute the point estimates of the parameters is increased. As in the other examples in this chapter, when the number of observations, $r$, used to compute the point estimates of the parameters increases, the expectation tends to expected number of customers $\bar{N}$, computed at point estimates of the parameters (which tends to the true value as the number of observations is increased). While the uncertainty propagation method allows different number of observations for different parameters, same value of $r$ is used for all the parameters, at each point in Figure 4.3, for ease of illustration. Similarly,

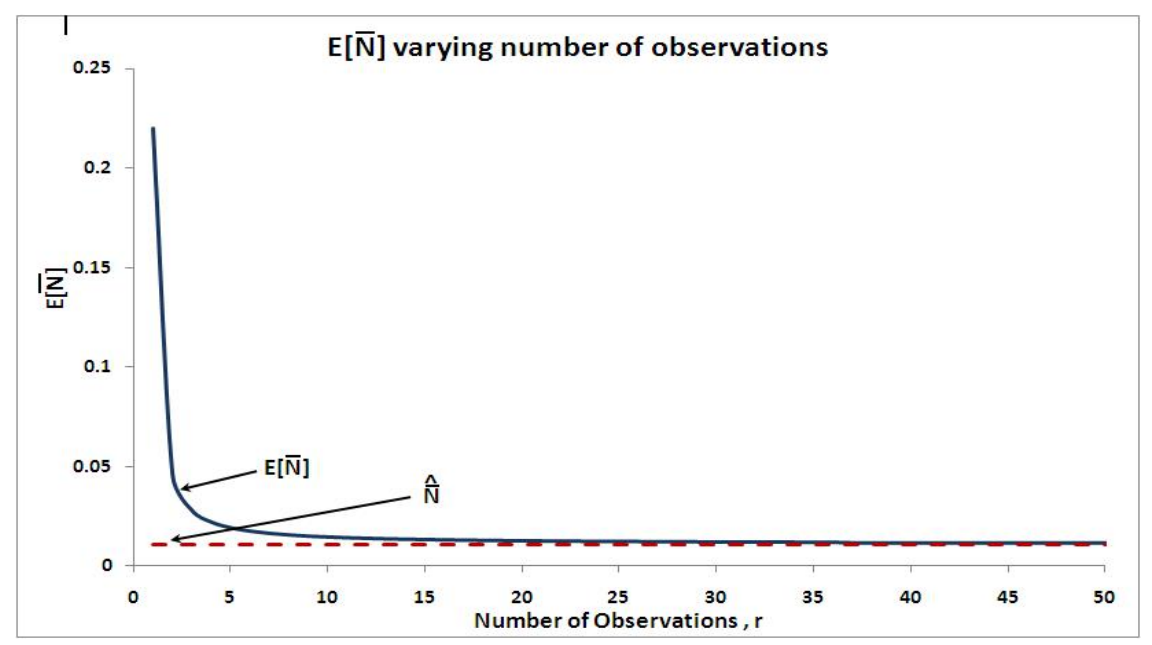

FiguRE 4.3: Expected value of Expected Number of Customers in the system

based on Equations 3.2 and 3.3, the variance of expected number of customers in the system, due to the epistemic uncertainty in model input parameters, is obtained. The computation of variance also requires using linearity property of variance of independent random variables $(\operatorname{Var}[A+B]=\operatorname{Var}[A]+\operatorname{Var}[B]$, where $A$ and $B$ are 
independent random variables) and properties of variance of product of two independent random variables $\left(\operatorname{Var}[A B]=(E[A])^{2} \operatorname{Var}[B]+(E[B])^{2} \operatorname{Var}[A]-\operatorname{Var}[A] \operatorname{Var}[B]\right.$, where $A$ and $B$ are two independent random variables). The variance thus computed (shown in Figure 4.4) tends to 0 as the number of observations used to compute the point estimate of $\bar{N}$ increases (indicating the point estimate of $\bar{N}$ approaching the true value).

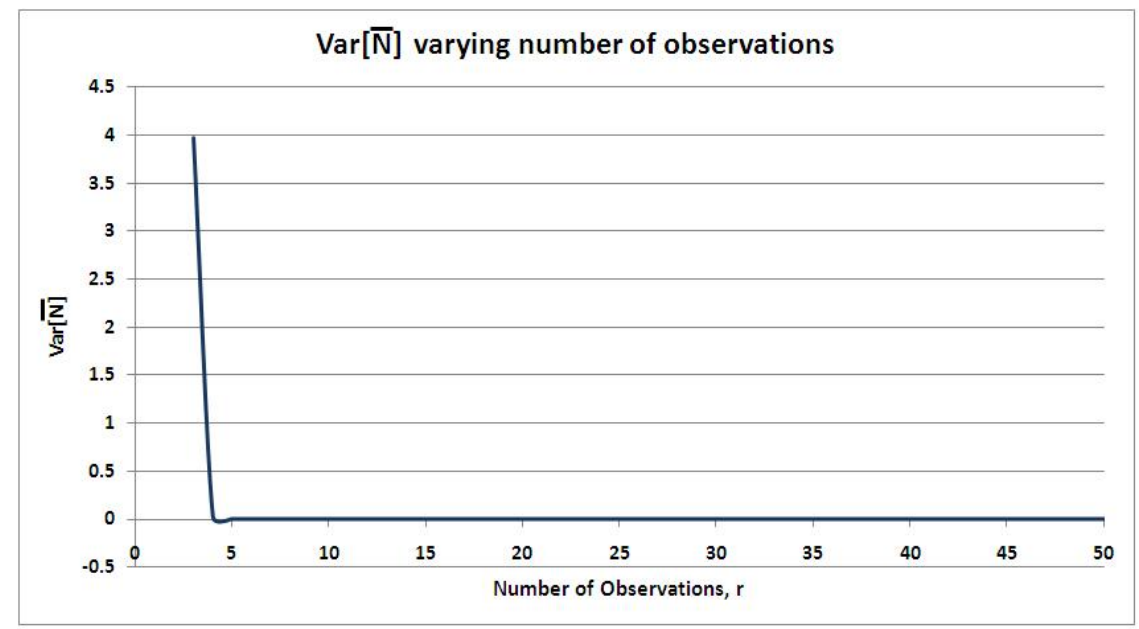

FiguRE 4.4: Variance of Expected Number of Customers in the system

\subsection{Probability of a Successful Attack}

We perform epistemic uncertainty propagation through an attack countermeasure tree (ACT) model, discussed in [71], as another illustration of analytic-numeric epistemic uncertainty propagation. An attack countermeasure tree is a new attack tree paradigm to analyze security of systems and evaluate measures of importance like return on investment for security countermeasures or a return on attack (the benefit to the attacker from a particular attack). The ACT takes into account both the attacks as well as the countermeasures (the attack detection and mitigation events). We consider the ACT model of the attack event of resetting a Border Gateway Protocol (BGP) routing session, described in [71]. The ACT model discussed in [71] is 
reproduced below in Figure 4.5 for ease of reference. The probability of occurrence of

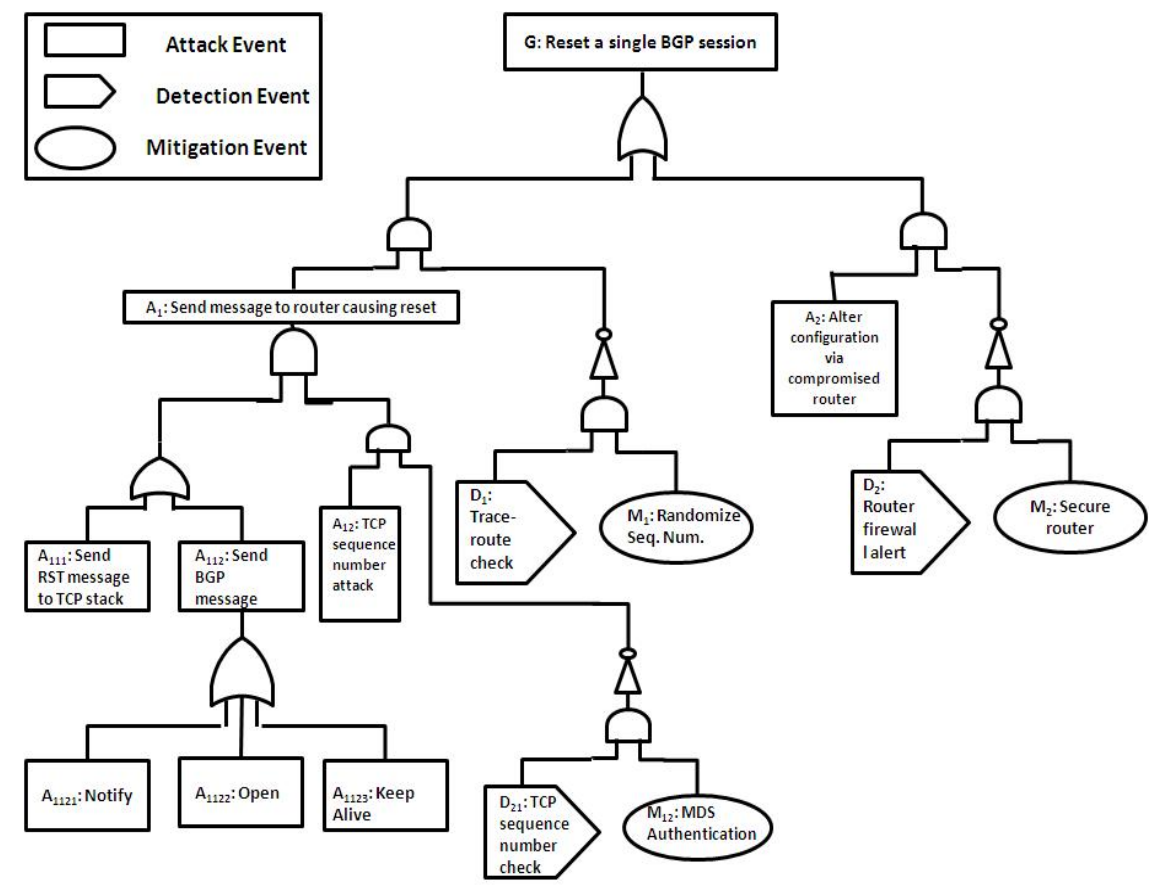

Figure 4.5: An ACT for resetting a BGP session

the top level event in the ACT gives the probability of a successful attack. Various detection and their corresponding mitigation events (countermeasures) have been considered in this model, as shown in Figure 4.5. Probability of successful attack, $P_{\text {goal }}$, is given by:

$$
\begin{aligned}
P_{\text {goal }}= & \left(p_{A_{111}}+p_{A_{1121}}+p_{A_{1122}}+p_{A_{1123}}\right) \times\left(p_{A_{12}} \times \overline{p_{D_{12}} \times p_{M_{12}}}\right) \\
& \times \overline{p_{D_{1}} \times p_{M_{1}}}+p_{A_{2}} \times \overline{p_{D_{2}} \times p_{M_{2}}}
\end{aligned}
$$

where, $p_{A_{i}}$ is the probability of an attack event $A_{i}$, while $p_{D_{i}}$ and $p_{M_{i}}$ are probabilities of the corresponding detection and mitigation events, respectively. We compute the expectation and variance of probability of a successful attack (resetting a BGP session), in presence of imperfect detection and mitigation of attacks. Based on Equation 3.2 and using the beta density from Equation 2.14, as the epistemic density of each of the input parameters, expectation and variance of $P_{\text {goal }}$ due to the epistemic 
uncertainties in the probabilities of various attack, detection and mitigation events, are computed using the Global Adaptive method of numerical integration and NIntegrate function of Mathematica [2]. Figure 4.6 shows the expectation of $P_{\text {goal }}$, as the number of observations that would have been used to compute the point estimates of the model input parameters, $r$, is increased. As seen in earlier examples, the expected probability of successful attack, $E\left[P_{\text {goal }}\right]$, tends to the point estimate $\hat{P}_{\text {goal }}$, as the number of observations increase.

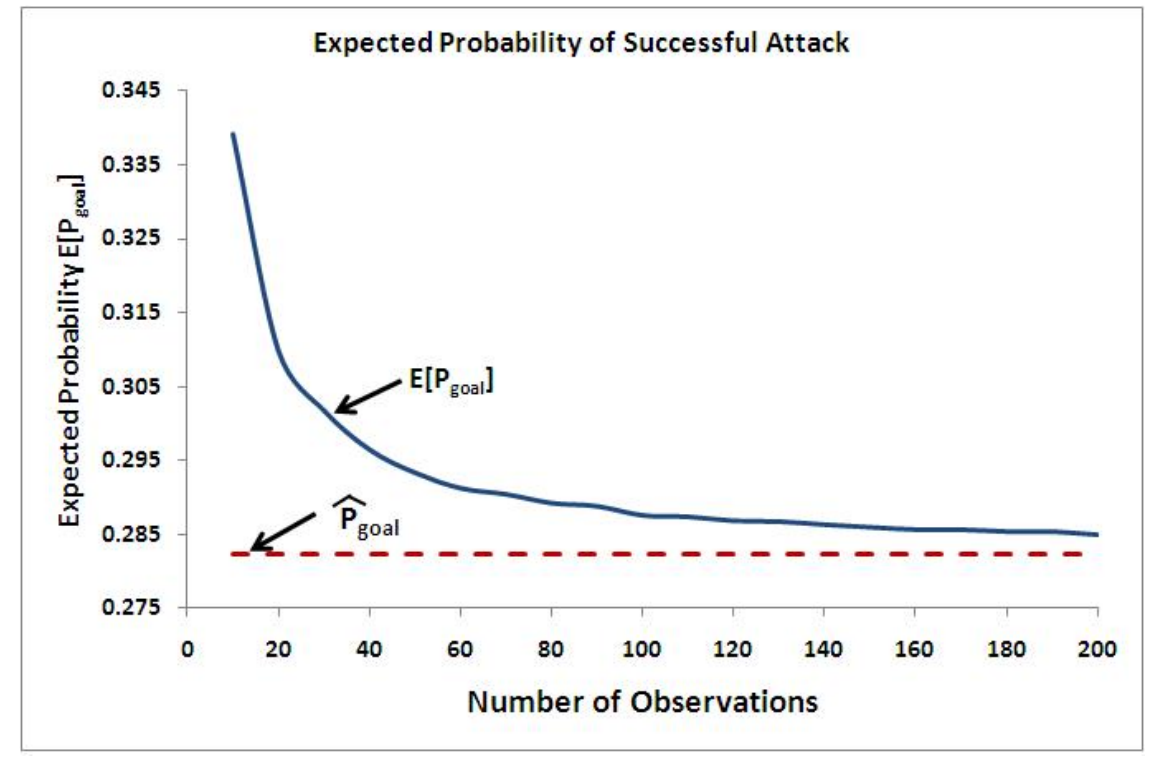

Figure 4.6: Expected Probability of Successful Attack, $E\left[P_{\text {goal }}\right]$ 


\section{5 Sampling Based Uncertainty Propagation}

Epistemic uncertainty propagation via unconditioning integrations as shown in Section 3.1, becomes difficult for complex aleatory models. Integration (analytical or numerical) of the aleatory model output expression quickly becomes intractable for complex models or models with several input parameters, as it becomes obvious in the examples shown in Chapters 3 and 4.

In this chapter, we present a sampling based method for epistemic uncertainty propagation through stochastic models to compute uncertainty in model output metrics. This method is more widely applicable than existing analytic methods. This

method does not require the output of the model to be a closed-form expression of input parameters and is easier to use for more complex models as it does not have to manipulate the model output. It is a sampling based unobtrusive method which acts as a wrapper to already existing stochastic models and their solution methods. Since this uncertainty propagation method does not require the model output to be a closed-form expression of the input parameters, it can be applied to model types ranging from simple non-state-space models (reliability block diagrams, fault trees etc. [87]) which assume independence of component failures and repairs, to more 
complex state space models like Markov chains, semi-Markov and Markov regenerative processes which capture dependence between events or stochastic Petri nets to capture large state space $[8,56,24,4,86,31]$. This method will also work with large hierarchical models [72] and fixed-point iterative models [87, 84].

The confidence interval of the aleatory model output metrics is obtained using a non-parametric method, obviating any need for assuming or fitting any distribution to the model outputs. The form and parameters of the epistemic distributions are not arbitrarily assumed (as is done in other sampling based methods as discussed in [28]), but derived based on the aleatory distribution and information about uncertainty in model input parameters (confidence intervals of the parameters or number of observation used to compute the estimates of the parameter) provided confidence intervals. The method of obtaining the form and parameters of epistemic distribution is also discussed. The details of this method and its illustration with the help of uncertainty analysis of dependability of some complex computer systems are discussed in the rest of this chapter ${ }^{1}$.

\subsection{Overview of the Method}

Considering the aleatory model output $M$ to be a function $g\left(\Theta_{1}, \Theta_{2}, \ldots, \Theta_{k}\right)$ of the $k$ model input parameters as epistemic random variables $\left\{\Theta_{i}, i=1,2, \ldots, k\right\}$, the computation of distribution, expectation and second moment (hence variance) by unconditioning integrations have been shown in Equations 3.1 through 3.3.

Although these integrals can be formally evaluated as shown in Chapters 3 and 4, they quickly become intractable for complex expressions of model output or for a large number of input parameters. As it was clear in examples towards the end of Chapters 3 and 4, integration (analytical or numerical) of expressions of aleatory model output was already getting intractable for even simple expressions of model

\footnotetext{
${ }^{1}$ Adapted from $[18,55]$
} 
outputs. Apart from the computational problem, the joint epistemic density of all the model parameters, also needs to be specified. In this chapter we assume that these epistemic random variables are independent and hence the joint density can be factored into the product of the marginals. Then, we employ a simple Monte Carlo approach to computing this integral. We later relax the assumption of independence by considering rank correlation between parameters in Chapter 7 . It should be noted that dependence can always be allowed in the aleatory model (e.g., dependence between failure and repair events in a system) by using state-space models, even when independence is assumed among the epistemic random variables (model input parameters), as explained in Section 2.4.

Evaluating $M\left(\theta_{1}, \theta_{2}, \ldots, \theta_{k}\right)$ at samples drawn from the joint probability distribution of all the input parameters, $F_{\Theta_{1}, \Theta_{2}, \ldots, \Theta_{k}}\left(\theta_{1}, \theta_{2}, \ldots, \theta_{k}\right)$, would yield a sample of values of the (conditional) model output metric. Under the assumption of independence among the parametric random variables, samples can be drawn independently from the probability distribution of individual parameters (marginal distributions). The sample of values of the model output metric thus obtained, can be analyzed to infer its distribution and hence the confidence interval.

Figure 5.1 summarizes the steps in the uncertainty propagation method.

We first determine the epistemic distribution and the parameters of epistemic distribution for each of the aleatory model input parameters. In the determination of epistemic distributions, we make use of the aleatory distribution and the number of observations (failures, repairs, recoveries etc.) that would have been used to estimate the parameters of the aleatory distribution. The number of samples to be drawn from the epistemic distributions is then computed and samples are drawn from the epistemic distribution of each of the aleatory model parameters. The set of output values obtained by solving the model at each set of sampled parameter values is finally analyzed to obtain the confidence interval of the model output metric 


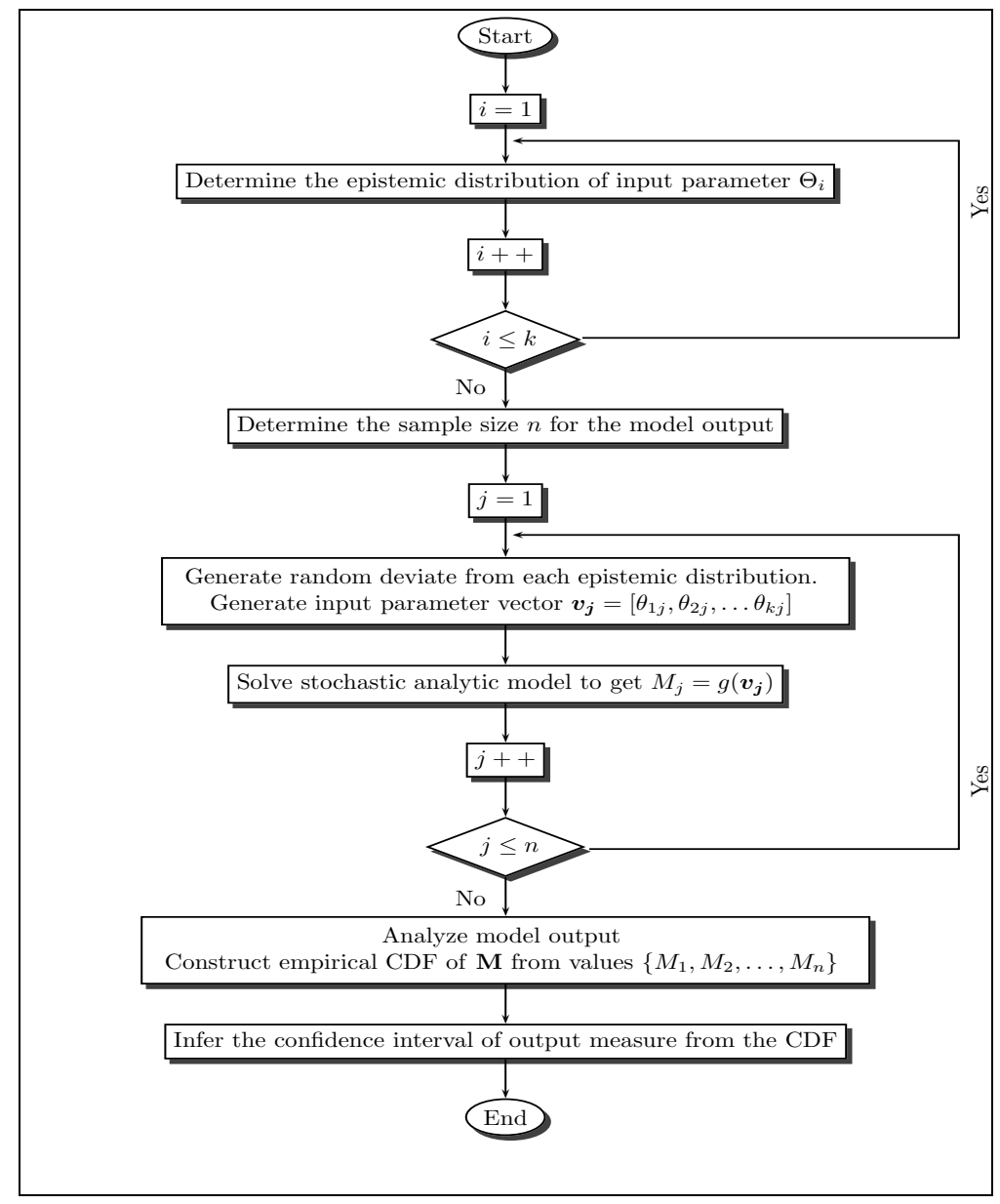

Figure 5.1: Flow Chart of Uncertainty Propagation Method

(unconditional availability). In this chapter, we discuss each of these steps in detail and then implement this method for parametric epistemic uncertainty propagation to compute the uncertainty in outputs of a dependability or a performance model of computer systems to illustrate its applicability. 


\subsubsection{Determining Epistemic Distributions}

Since this method is based on sampling from the epistemic distributions of the model parameters, we first need to determine the epistemic distribution (types and their parameters) of the model input parameters, from the uncertainty in the input parameter value and the aleatory distribution type. If the lifetime data is available, we determine epistemic distributions (types and their parameters) from the observed data.

As shown in Section 2.3, the posterior distribution obtained by applying Bayes' theorem to the likelihood of input parameters (based on the aleatory distributions) and an appropriate prior [23], provides the epistemic distribution of parameters of the aleatory distribution [83].

The random deviates generated from the epistemic density derived in Equation 2.6 are used in our uncertainty propagation method as values of the parameters of the aleatory model. It is clear from Equation 2.6 that the epistemic density thus derived depends on the aleatory distribution and will be different for different aleatory distributions. In the examples discussed in this chapter, the exponential aleatory distribution (for times to failure and times taken by different recovery actions, response times etc.) and the Bernoulli aleatory distribution (for ability to detect or provide coverage of faults) are assumed. The form and parameters of epistemic distributions for parameters of exponential and Bernoulli aleatory distribution are derived in Sections 2.3.2 and 2.3.3, respectively and are reproduced here for ease of reference.

The epistemic $p d f$ of the rate parameter (as random variable) of the exponential aleatory distribution, $\Theta_{i}$ ( $i^{\text {th }}$ parameter of the aleatory model, say rate of failure), is given by:

$$
f_{\Theta_{i}}\left(\theta_{i}\right)=\frac{\theta_{i}^{r_{i}-1} s^{r_{i}} e^{-\theta_{i} s}}{\left(r_{i}-1\right) !}=\text { Erlang } p d f\left(r_{i} ; s\right)
$$


where, $s$ is the value of the sum of observed times to failure random variable $S$ and $r_{i}$ is the number of observed failures, used to compute the point estimate of $\theta_{i}$. Equation 5.1 has been obtained using Jeffrey's prior of $\Theta_{i}$, as $f_{\Theta_{i}}\left(\theta_{i}\right)=s / \theta_{i}[80]$. With a uniform prior, the epistemic $p d f$ of parameter of exponential distribution comes out to be Erlang $p d f\left(r_{i}+1 ; s\right)$. In the examples in this chapter we use Erlang $p d f\left(r_{i} ; s\right)$ as the epistemic $p d f$ for the rate parameter of exponential aleatory distribution.

If the $j^{\text {th }}$ parameter $\theta_{j}$, of the reliability model, is a coverage parameter (say, the probability of successfully handling a fault) derived from a fault injection experiment, introducing $r_{j}$ faults. The epistemic $p d f$ of $\Theta_{j}, f_{\Theta_{j}}\left(\theta_{j}\right)$ is derived in Section 2.3.3 and is reproduced below for ease of reference in this chapter :

$$
f_{\Theta_{j}}\left(\theta_{j}\right)=\frac{\left(\begin{array}{l}
r_{j} \\
y_{j}
\end{array}\right) \theta_{j}^{y_{j}}\left(1-\theta_{j}\right)^{r_{j}-y_{j}}}{\int_{0}^{\infty}\left(\begin{array}{l}
r_{j} \\
y_{j}
\end{array}\right) \theta_{j}^{y_{j}}\left(1-\theta_{j}\right)^{r_{j}-y_{j}} d \theta_{j}}=\underbrace{\frac{\Gamma\left(r_{j}+2\right)}{\Gamma\left(y_{j}+1\right) \Gamma\left(r_{j}-y_{j}+1\right)} \theta_{j}^{y_{j}}\left(1-\theta_{j}\right)^{r_{j}-y_{j}}}_{\text {beta pdf }\left(y_{j}+1 ; r_{j}-y_{j}+1\right)}
$$

where, the random variable $Y_{j}$ denotes the number of successfully covered faults out of $r_{j}$ faults injected,

If the lifetime data or data from fault injection experiments, used to compute the input parameter values, is directly available, it can be used to obtain the parameters of the above epistemic distributions $\left(r_{i}\right.$ and estimate for $s$ for Equation $5.1 ; r_{j}$ and $y_{j}$ for Equation 5.2 can be obtained/computed directly from the observed data). If the modeling expert does not have direct access to observed data and the epistemic uncertainty of parameters is available only in the form of confidence intervals of parameters (which is the case assumed in the illustrative examples in this chapter), this method of determining the epistemic distribution requires knowledge of the aleatory distribution type and the number of observations that would have been used to infer the confidence interval of the parameter. 
In this section, we first discuss computing the number of observations, $r_{i}$, that would have been used to compute the point estimate and confidence interval of the $i^{\text {th }}$ model parameter, $\theta_{i}(i=1,2, \ldots, k)$ (when observed data is not directly available). Aleatory distributions for the times to failure (exponential) or the ability to successfully detect or recover from failures (Bernoulli) have been considered. Using the computed $r_{i}$ and the point estimate of the parameter, the epistemic distributions for each of the model parameters are determined based on Equations 2.10 and 2.14.

In the rest of the chapter we will consider $r_{i},(i=1,2, \ldots, k)$, to be the number of observations that would have been used to compute the point estimate and confidence interval of the model input parameter $\theta_{i}$ (parameter of the aleatory distribution). However, in the remaining parts of this section, when determining the number of observations for different aleatory distribution types, we denote it simply as $r$.

\section{Computation of number of observations}

We compute the number of observations by inverting the relation between the width of the confidence interval and the number of observations and make use of the point estimate of the input parameter. In case the point estimate of the parameter is not provided and only the confidence interval is provided, the mid point of the confidence interval is assumed to be the point estimate, for the purpose of this calculation. We consider determining the number of observations when the aleatory distribution of times to failure and repair follow an exponential distribution and the aleatory distribution of the indicator of successfully detecting or recovering from a failure follow a Bernoulli distribution.

Exponential distribution : Number of observations from two-sided con-

\section{fidence interval}

When the time to failure or repair of a component is exponentially distributed, the

point estimate of the rate parameter $\hat{\lambda}$ of the exponential distribution is given by 
$\hat{\lambda}=r / s_{r}$, where, $r$ is the number of observed failures during the period of observation and $s_{r}$ is the value of accumulated life on test random variable $S_{r}$. Making use of the upper and lower limits of $100(1-\alpha) \%$ two sided confidence interval of $\lambda[67,86]$, the half width of the confidence interval of $\lambda$, given by $d$, can be determined as:

$$
d=\frac{1}{4 s_{r}}\left\{\chi_{2 r, \alpha / 2}^{2}-\chi_{2 r, 1-\alpha / 2}^{2}\right\}
$$

where, $\chi_{2 r, 1-\alpha / 2}^{2}$ is the critical value of chi-square distribution with $2 r$ degrees of freedom. From Equation (5.3), the number of observations (number of observed failures, repairs etc.) that would have resulted in a given $d$ at a confidence coefficient $(1-\alpha)$ can be computed as:

$$
r=\left\lceil\frac{\hat{\lambda}}{4 d}\left\{\chi_{2 r, \alpha / 2}^{2}-\chi_{2 r, 1-\alpha / 2}^{2}\right\}\right\rceil
$$

Starting with an initial assumption for the value of $r$, fixed-point Equation (5.4) is solved by successive substitution to converge at a value of $r$.

\section{Exponential distribution : Number of observations from one-sided con-}

\section{fidence interval}

Using the lower limit of the $100(1-\alpha) \%$ upper one-sided confidence limit of $\lambda[67,86]$, the number of observations, $r$, that would have resulted in this lower limit can be computed as:

$$
r=\left\lceil\frac{\hat{\lambda}}{\lambda_{L}}\left\{\chi_{2 r, 1-\alpha}^{2}\right\}\right\rceil
$$

where $\lambda_{L}$ is the lower limit of the upper one-sided confidence interval. First we generate an approximate answer using the normal approximation of the chi-square distribution. Then we bisection to calculate the exact value of $r$, using half and two times the approximate value as the initial bounds. Similarly, we can get the number 
of observations for the $100(1-\alpha) \%$ lower one-sided limit to be:

$$
r=\left\lceil\frac{\hat{\lambda}}{\lambda_{U}}\left\{\chi_{2 r, \alpha}^{2}\right\}\right\rceil
$$

where $\lambda_{U}$ is the upper limit of the $100(1-\alpha) \%$ lower one-sided confidence interval.

\section{Bernoulli distribution : Coverage with one-sided confidence interval}

The point estimate of the coverage probability is given by $\hat{c}=s_{r} / r$, where $S_{r}$ is the random variable denoting the number of faults/errors detected or recovered, $s_{r}$ is its value and $r$ is the total number of faults/errors injected. The lower limit of the upper one sided $100(1-\alpha) \%$ confidence interval for the coverage probability is given by Equation (5.7) [86]:

$$
c_{L}=1-\frac{\chi_{2\left(r-s_{r}+1\right), \alpha}^{2}}{2 r}
$$

Inverting Equation (5.7), the number of injections that would have resulted in this lower limit of the confidence interval, $c_{L}$, at a confidence coefficient $(1-\alpha)$ can be obtained as:

$$
r=\left\lceil\frac{\chi_{2(r(1-\hat{c})+1), \alpha}^{2}}{2\left(1-c_{L}\right)}\right\rceil
$$

Equation (5.8) is iteratively solved using successive substitution to obtain the value of $r$, starting with an initial approximation calculated using the normal approximation of the chi-square distribution.

\subsubsection{Determining the Sample Size}

After determining the marginal epistemic density function for each of the parameters, the total number of samples $n$, to be drawn from each of them, needs to be determined. The total number of samples is computed as $n=\max \left\{r_{1}, r_{2}, \ldots, r_{k}, m_{1}, m_{2}, \ldots, m_{l}\right\}$, where $r_{i}$ is the number of observations that would have been used to compute 
the point estimate and confidence interval of each model input parameter $\Theta_{i}(i=$ $1,2, \ldots, k)$ and $m_{o}(o=1,2, \ldots, l)$ is the sample size needed for the output measures, $M_{o}$. The sample size based on the output measure(s) is considered only if a desired confidence interval of any of the output measures is provided. Calculation of sample size for model output measures

If the output measure follows a normal distribution $N(\mu, \sigma)$, then using $100(1-\alpha) \%$ two sided confidence interval for the mean $\mu$, the half width of the confidence interval of $\mu$ is given by Equation (5.9) [86]:

$$
d=\frac{t_{m_{o}-1, \alpha / 2} s}{\sqrt{m_{o}}}
$$

where, $m_{o}$ is the sample size of the output metric, $t_{m_{o}-1, \alpha / 2}$ is a critical value of the Student's $t$ - distribution with $m_{o}-1$ degrees of freedom, $\bar{x}$ is the value of sample mean and $s$ is the value of sample standard deviation of the output measure. Inverting Equation (5.9), the number of samples needed for the half-width $d$, at a confidence coefficient $(1-\alpha)$ can be obtained as:

$$
m_{o}=\left\lceil\left\{\frac{t_{m_{o}-1, \alpha / 2} s}{d}\right\}^{2}\right\rceil
$$

Starting with an initial approximation for $m_{o}$, Equation (5.10) is iteratively solved to obtain the value for $m_{o}$. The initial approximation for $m_{o}$ is obtained by replacing the critical values of $t$-distribution in Equation (5.10) with critical values of standard normal distribution. This method can also be applied for determining number of samples if the output measure followed a lognormal distribution. Similar reasoning can be used if the one-sided confidence interval is provided. The lower limit on the $100(1-\alpha) \%$ upper one-sided confidence interval for parameter $\mu$ is given by:

$$
\mu_{L}=\bar{x}-\frac{t_{m_{o}-1, \alpha} s}{\sqrt{m_{o}}}
$$


The half width, $d$, is thus given by:

$$
d=\frac{t_{m_{o}-1, \alpha} s}{\sqrt{m_{o}}}
$$

Inverting Equation (5.12), the number of samples needed for the half-width $d$, at a confidence coefficient $(1-\alpha)$ can be obtained :

$$
m_{o}=\left\lceil\left\{\frac{t_{m_{o}-1, \alpha} s}{d}\right\}^{2}\right\rceil
$$

The sample size for the $100(1-\alpha) \%$ lower one sided confidence interval is also given by Equation (5.13). Equation (5.13) is iteratively solved to obtain the value for $m_{o}$, starting with an initial approximation for $m_{o}$ is obtained by replacing the critical values of $t$-distribution in Equation (5.13) with critical values of standard normal distribution. This method can also be applied for determining number of samples if the output measure followed a lognormal distribution.

\subsubsection{Sampling Procedure}

Once the epistemic distribution and its parameters are determined for each model input parameter and the total number of samples $n$, needed from each epistemic distribution, is calculated as in Section 5.1.2, samples of random deviates from the epistemic distribution of each parameter need to be obtained next. Random sampling from epistemic distributions of the model input parameters is performed. The samples of random numbers or the random deviates can be generated by any number of methods such as the inverse transform method [86], the Box-Mueller transform [9] or rejection sampling [88]. A sample from the distribution of each of the $k$ model input parameters will result in a vector of $k$ parameter values. Since, $n$ samples are needed from each distribution, $n$ such vectors $\left(\boldsymbol{v}_{\mathbf{1}}\right.$ through $\left.\boldsymbol{v}_{\boldsymbol{n}}\right)$ will be generated, as shown in Equation (5.14). 
The actual samples of random numbers or the random deviates can be generated by any of the methods like inverse transform method, rejection sampling, Box-Mueller transform or Johnson's translation [37].

A sample from the distribution of each of the $k$ model input parameters will result in a vector of $k$ parameter values. If $n$ samples are drawn from each distribution, an $n-b y-k$ matrix can be generated. The elements of each column in this matrix are then randomly rearranged. This rearrangement is necessary to ensure randomness in order of samples obtained by LHS sampling procedure. Each of the $n$ rows of the matrix $\boldsymbol{V}$, after rearrangement, can be considered as $n$ vectors $\left(\boldsymbol{v}_{\mathbf{1}}\right.$ through $\left.\boldsymbol{v}_{\boldsymbol{n}}\right)$ of model input parameter values as:

$$
\boldsymbol{V}=\left[\begin{array}{c}
\boldsymbol{v}_{\mathbf{1}} \\
\boldsymbol{v}_{\mathbf{2}} \\
\vdots \\
\boldsymbol{v}_{\boldsymbol{n}}
\end{array}\right]=\left[\begin{array}{c}
\theta_{11}, \theta_{21}, \ldots \theta_{k 1} \\
\theta_{12}, \theta_{22}, \ldots \theta_{k 2} \\
\vdots \\
\theta_{1 n}, \theta_{2 n}, \ldots \theta_{k n}
\end{array}\right]
$$

\subsubsection{Analytic Model Solution}

The stochastic analytic model is solved at each of the $n$ sets of samples of input parameter values obtained from their epistemic distributions. Solving the model at each of these sets of input parameter values, yields a set of $n$ values of the model output metric, as:

$$
\left\{M_{1}, M_{2}, \ldots, M_{n}\right\}=\left\{g\left(\boldsymbol{v}_{\mathbf{1}}\right), g\left(\boldsymbol{v}_{\mathbf{2}}\right), \ldots, g\left(\boldsymbol{v}_{\boldsymbol{n}}\right)\right\}
$$

This approach is not dependent on the model solution method nor does it require the model outputs to be a closed-form expression of the input parameters, as it does not manipulate the model or its solution. The models may range from simple non-state-space models like fault trees and reliability block diagrams, which can capture independent failures and repairs to state space model like Markov chains and stochastic reward nets which capture dependency between events to complex 
multi-level hierarchical models which address largeness of state-space and/or stiffness (presence of rare events or very fast events). The model may be solved by using software packages like SHARPE [87] or SPNP [32] or if closed-form solutions exist, they may be solved programatically by simple user programs. Our technique simply provides a wrapper around the existing aleatory model solver.

\subsubsection{Summarizing the Output}

Using the set of model output values $\left\{M_{1}, M_{2}, \ldots, M_{n}\right\}$ we quantify the uncertainty in the output due to the epistemic uncertainties in model input parameters, by computing the confidence interval of the output metric(s), at a desired confidence level. In the confidence interval computation, we use a non-parametric method to avoid having to make any assumptions about the distribution of the model output or fit a distribution to the model output values. An empirical Cumulative Distribution Function (CDF) [86] of the set of output values $\left\{M_{1}, M_{2}, \ldots, M_{n}\right\}$, is constructed. The values of the output measure at appropriate percentile points from the CDF, provide the confidence interval at the desired confidence level. In our examples, we use the values of the model output corresponding to $2.5^{\text {th }}$ and $97.5^{\text {th }}$ percentile, as the limits of the $95 \%$ two sided confidence interval. Similarly the value corresponding to $5^{\text {th }}$ percentile is chosen as the lower limit of the $95 \%$ upper one sided confidence interval and the value corresponding to the $95^{\text {th }}$ percentile is chosen as the upper limit of the $95 \%$ lower one sided confidence interval.

\subsection{Illustrative Examples}

We illustrate the epistemic uncertainty propagation method discussed in this chapter via examples of some real-world systems. These examples illustrate that this uncertainty propagation method can be applied in a non-obtrusive way to a wide range of stochastic model types and is independent of the model solution technique. The 
dependability models in these examples range from a CTMC model of a composite web service with a closed-form output of the model to a large hierarchical availability model of a complex clustered server system with 63 model input parameters, for each a closed-form solution does not exist. The model output metrics of the aleatory models considered are availability, reliability and mean response time. The uncertainty propagation wrapper is implemented in Mathematica, while the models are solved using SHARPE [87] and Mathematica [2]. The remainder of this chapter describes these example systems and their models and illustrates epistemic uncertainty propagation through these stochastic models.

\subsubsection{Availability of IBM Bladecenter Server System}

We use the availability computation of the IBM Bladecenter system discussed in [82], as an example for the numerical illustration of this method. First we briefly introduce the example system and then go through each of the steps in the uncertainty propagation method. We also discuss the intermediate outputs (along with the parameter values) and finally summarize the results of uncertainty propagation and compute the confidence interval of system availability.

The IBM BladeCenter system [82] consists of up to fourteen computing elements known as blade servers in one chassis. The chassis consists of redundant blowers (cooling system), two power domains each containing two redundant power supply modules, redundant management modules, redundant Ethernet switches, and redundant Fiber Channel network switches. These subsystems are shared by all the blade servers in the chassis. Redundant subsystems exist inside each blade server as well. Each of the subsystems or components have been modeled as Markov chains with exponentially distributed times to failure and repair. Markov chain models for availability of chassis components (midplane, cooling subsystem, power domain and network subsystem) and blade server components (blade server, processor subsys- 
tem, software, memory subsystem and disk storage subsystem) are constructed. The overall availability of the system is modeled using a hierarchical model. The top level model is a fault tree, with the unavailability of each subsystem, as its input. Repeated events are used in the fault tree to capture failures due to shared components or subsystems. For details of these models, the reader is referred to the paper [82], in IBM Systems Journal. The top-level fault tree model is reproduced below in Figure 5.2 for ease of reference.

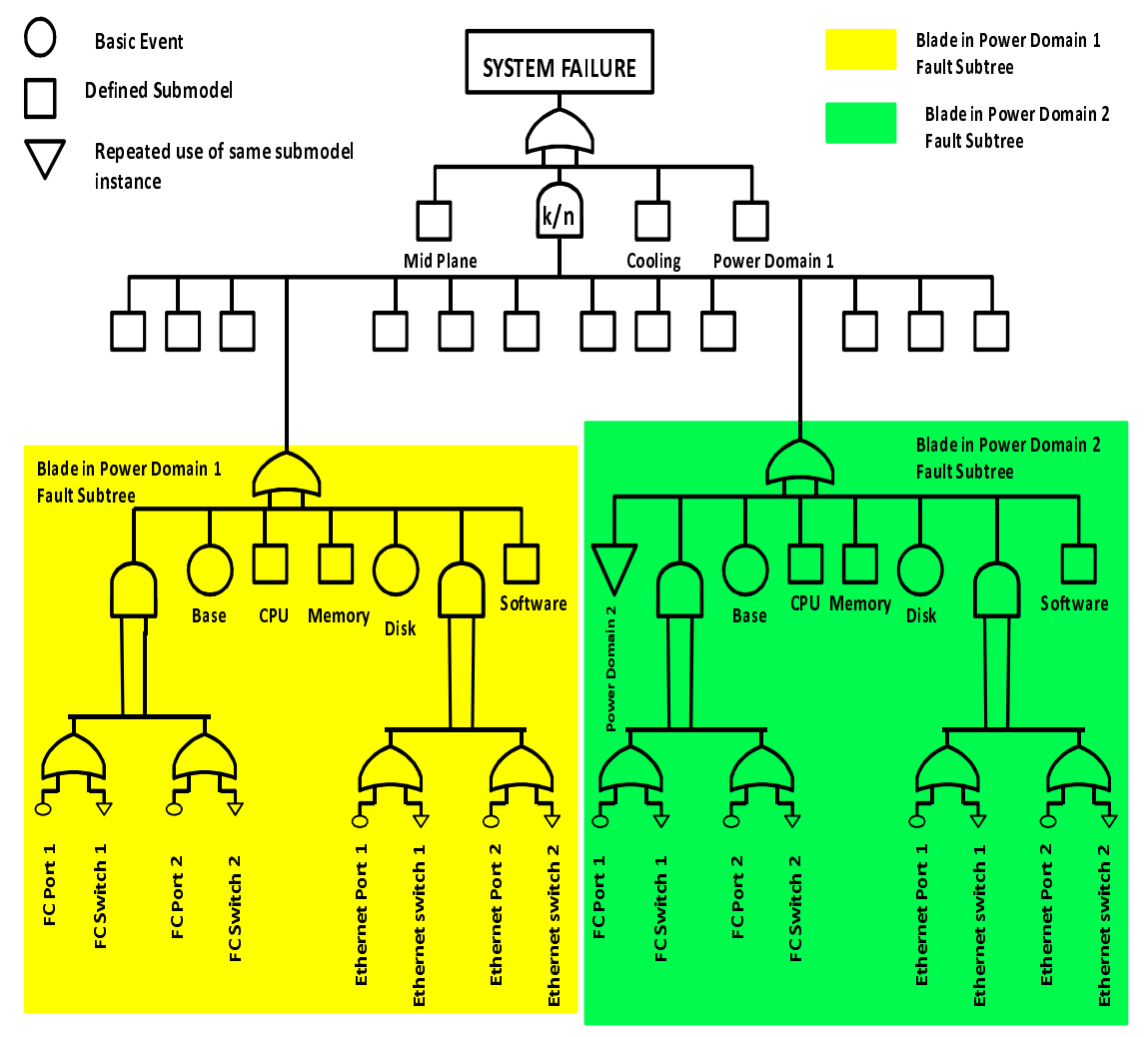

Figure 5.2: Top level fault tree of BladeCenter availability Model

The steps in propagating the uncertainty in parameter values through the availability model for the IBM BladeCenter are as follows :

(a) Determining the epistemic distributions of model parameters : We 
first determine the number of observations that would have been used to compute the point estimate and $95 \%$ confidence interval, for each model parameter, as explained in Section 5.1.1. The number of observations, thus calculated for each model input parameter, is summarized in Table 5.1. To illustrate the iterative process involved in computing the number of observations, consider calculating the number of observations of $1 / m t t f f c s w$ which has a point estimate $2.6247 e-06$ and halfwidth $3.517 e-07$ at $95 \%$ confidence level. We begin with an initial approximation for the number of observations as 50 (an arbitrarily chosen number) and iteratively generate the number of observations to be $104,150,180,197,206,210,212,213$. We stop iterating when we converge on the number of observations (when the number of observations of two successive iterations differ by 1). The number of observations for $1 / m t t f f c s w$ is thus computed to be 213 .

The next step is to determine the epistemic distribution of each parameter by generating the posterior distributions. Since the subsystems have been modeled as Markov chains, the time to failure and time to repair have been modeled as exponential distributions. The coverage factors have been modeled using Bernoulli distribution. Therefore, as explained in Section 5.1.1, the posterior distribution of the failure and repair parameters is Erlang distribution and the coverage parameters have a beta posterior distribution. The parameters of the posterior distribution have been obtained from the hardware and software input parameters section of [82] and are summarized in Table 5.1.

(b) Determination of number of samples from epistemic distributions : The number of samples needed from the distribution of each input parameter is determined to be $n=901$, largest of all the number of observations of the input parameters.

(c) Generating Random Deviates from the epistemic distributions : The samples from the Erlang and beta distributions for the parameters are generated 
Table 5.1: Parameters of the Posterior Distributions

\begin{tabular}{|c|c|c|}
\hline Parameter & $\begin{array}{l}\text { Num. Obser- } \\
\text { vations }\end{array}$ & $\begin{array}{l}\text { Parameter Values of } \\
\text { Posterior }\end{array}$ \\
\hline $1 / m t t f h d d$ & 17 & Erlang(17,5.1e06) \\
\hline $1 / m t t r h d d$ & 44 & Erlang $(44,22)$ \\
\hline $1 / m t t r s p$ & 383 & Erlang $(383,957.5)$ \\
\hline $1 / m t t f s w$ & 383 & Erlang $(383,6.7 \mathrm{e} 06)$ \\
\hline $1 / m t t$ boot 1 & 383 & Erlang $(383,126.4)$ \\
\hline $1 /$ mttboot 2 & 383 & Erlang(383,287.3) \\
\hline $1 / m t t r s w$ & 383 & Erlang $(383,766)$ \\
\hline $1 / m t t r f c s w$ & 62 & Erlang $(62,31)$ \\
\hline $1 / m t t f f c s w$ & 213 & Erlang $(213,8.1 \mathrm{e} 07)$ \\
\hline $1 /$ mttrmem & 62 & Erlang $(62,31)$ \\
\hline $1 / \mathrm{mttfmem}$ & 110 & Erlang(110,6.28e07) \\
\hline $1 / m t t r c p u$ & 62 & Erlang $(62,31)$ \\
\hline $1 / m t t f c p u$ & 136 & Erlang $(136,4 \mathrm{e} 08)$ \\
\hline $1 /$ mttrps 1 & 71 & Erlang $(71,41.42)$ \\
\hline $1 /$ mttrps & 62 & Erlang $(62,31)$ \\
\hline $1 / m t t f p s$ & 124 & Erlang(124,9.76e07) \\
\hline $1 / m t t r c$ & 62 & Erlang $(62,31)$ \\
\hline $1 / m t t r 2 c$ & 71 & Erlang $(7,41.42)$ \\
\hline $1 / m t t f c$ & 122 & Erlang $(122,4.47 \mathrm{e} 08)$ \\
\hline $1 / m t t f m p$ & 125 & Erlang(125,4.51e07) \\
\hline $1 / m t t r m p$ & 62 & Erlang $(62,31)$ \\
\hline $1 /$ mttfbase & 102 & Erlang $(102,2.67 \mathrm{e} 07)$ \\
\hline $1 /$ mttrbase & 62 & Erlang $(62,31)$ \\
\hline $1 / m t t f e s w$ & 117 & Erlang(117,1.66e07) \\
\hline $1 /$ mttresw & 62 & Erlang $(62,31)$ \\
\hline $1 /$ mttfniceth & 121 & Erlang $(121,8.83 \mathrm{e} 08)$ \\
\hline $1 /$ mttrniceth & 62 & Erlang $(62,31)$ \\
\hline $1 / m t t f n i c f c$ & 101 & Erlang $(101,1.57 \mathrm{e} 08)$ \\
\hline $1 /$ mttrnicfc & 62 & Erlang $(62,31)$ \\
\hline$c 1$ & $\begin{array}{l}\text { faults injected: } \\
224 \text { faults de- } \\
\text { tected: } 190\end{array}$ & $\operatorname{beta}(191,35)$ \\
\hline$c 2$ & $\begin{array}{l}\text { faults injected: } \\
115 \text { faults de- } \\
\text { tected: } 109\end{array}$ & $\operatorname{beta}(110,7)$ \\
\hline$c p t$ & $\begin{array}{l}\text { faults injected: } \\
36 \text { faults de- } \\
\text { tected: } 35\end{array}$ & $\operatorname{beta}(36,2)$ \\
\hline cpsub & $\begin{array}{l}\text { faults injected: } \\
92 \text { faults de- } \\
\text { tected: } 91\end{array}$ & $\operatorname{beta}(92,2)$ \\
\hline $1-f m$ & $\begin{array}{l}\text { faults injected: } \\
901 \text { faults de- } \\
\text { tected: } 897\end{array}$ & $\operatorname{beta}(898,5)$ \\
\hline
\end{tabular}


using Mathematica's [2] built-in function RandomReal. For comparison with the method explained in [28], samples are also drawn from uniform distribution, $U(a, b)$, where $a$ and $b$ are the lower and upper bounds of the two-sided confidence interval of the parameter, provided. In case the upper one-sided confidence interval of the parameter is provided, $b$ is chosen to be 1 .

(d) Solving the Analytic Model : At each of the $n=901$ set of values of the parameters, the hierarchical model is solved using SHARPE [87].

(e) Summarizing the Output Values : The empirical Cumulative Distribution Function (CDF) of the output by both methods is constructed from the $n$ values of output, obtained by solving the system availability model using SHARPE. Figure 5.3 shows the empirical CDF of the availability of the BladeCenter System obtained by our method as well as by the method explained in [28] (sampling from uniform distribution), when there were no standby Blade Servers (all 14 Blade servers needed for system to be operational). Similar CDFs were constructed for availability of the BladeCenter system for different number of standby Blade Servers and number of Blade Servers needed for system to be considered operational. Figure 5.3 shows that sampling from uniform distribution for parameter values, provides a more optimistic and slightly wider confidence interval. The values for availability at higher percentile points seem to be higher than what our method suggests, in this example. Ignoring the shape of real epistemic distribution of a parameter and sampling from a uniform distribution, implies not using enough samples from regions of high probability and hence not representing the sample space correctly. Therefore, the distribution of model output thus generated may not represent the real distribution.

The $5^{\text {th }}$ percentile from the CDF provides the lower limit for the $95 \%$ upper one-sided confidence interval of availability. The upper and lower limits of the twosided $95 \%$ confidence interval of availability are provided by the $97.5^{\text {th }}$ percentile and $2.5^{\text {th }}$ percentile, respectively. The 95\% upper one-sided and two-sided confidence in- 


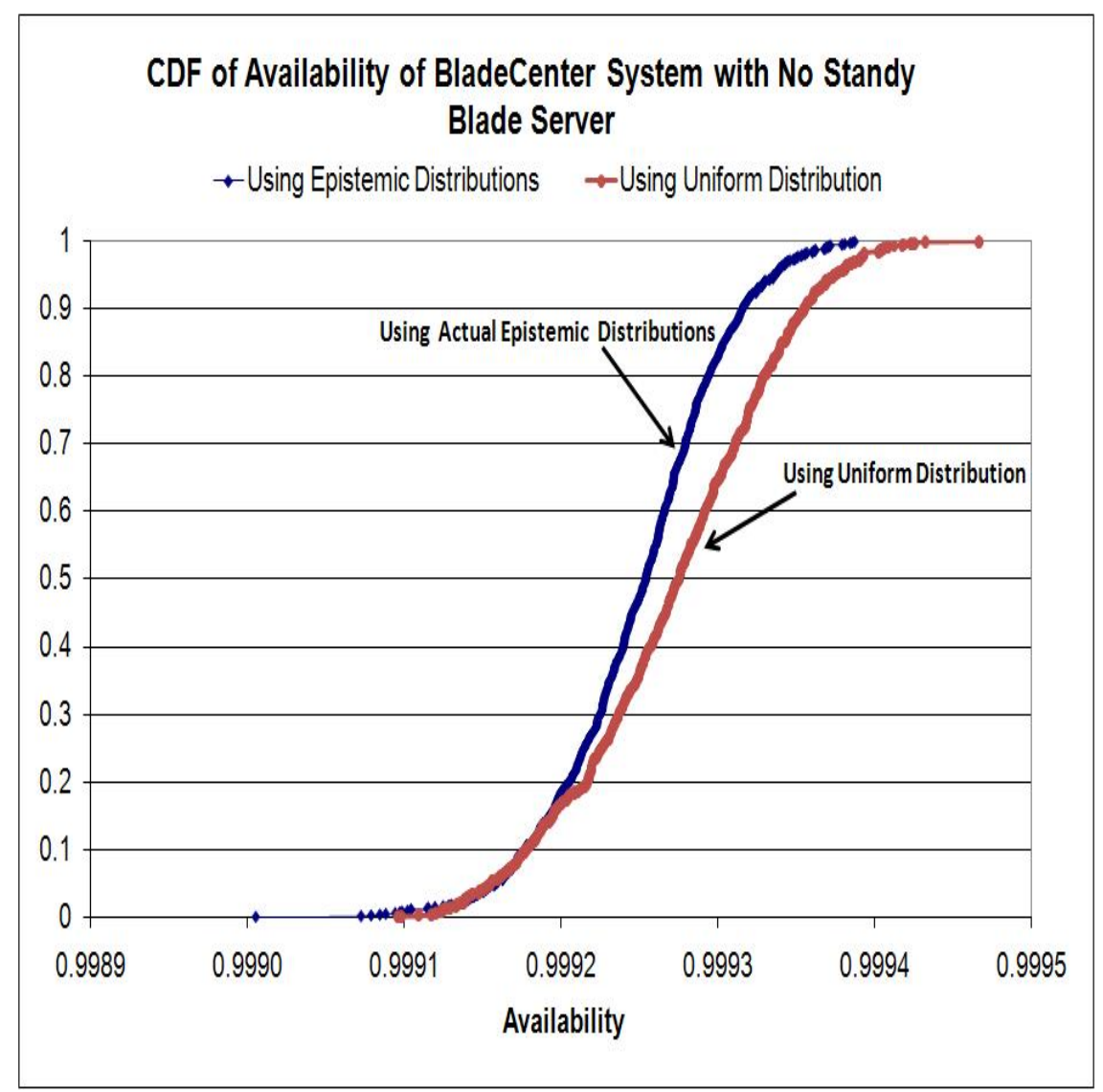

Figure 5.3: CDF of Blade Center Availability : No Standby Blade Server

tervals of availability of the BladeCenter system for different number of stand-by Blade Servers by the uncertainty propagation methods in this chapter, have been summarized in Table 5.2. Table 5.3 summarizes the confidence intervals and mean of availability, when parameter values are sampled from uniform distribution as discussed in [28].

\subsubsection{Availability Model of IBM SIP SLEE Server Cluster System}

We use the availability computation of the IBM SIP SLEE application server cluster system discussed in [85], as the next example for the numerical illustration of the uncertainty propagation method. The IBM SIP application server cluster is configured out of two IBM BladeCenter systems, with four blades (nodes) each. It employs both 
Table 5.2: Confidence Interval of Blade Center Availability

\begin{tabular}{|l|l|l|l|l|}
\hline $\begin{array}{l}\text { Num. } \\
\text { Reqd. }\end{array}$ & $\begin{array}{l}\text { Num. } \\
\text { Standby }\end{array}$ & Mean & $\begin{array}{l}95 \% \text { Upper } \\
\text { One-Sided } \\
\text { Conf. Int. }\end{array}$ & $\begin{array}{l}\text { 95\% Conf. Int. } \\
\text { Coided }\end{array}$ \\
\hline 14 & 0 & 0.9992495 & $0.9991582,1$ & $0.9991384,0.9993499$ \\
\hline 13 & 1 & 0.9999980 & $0.9999974,1$ & $0.9999972,0.9999985$ \\
\hline 12 & 2 & 0.9999982 & $0.9999976,1$ & $0.9999975,0.9999988$ \\
\hline 9 & 5 & 0.9999982 & $0.9999976,1$ & $0.9999975,0.9999988$ \\
\hline 6 & 8 & 0.9999984 & $0.9999980,1$ & $0.9999979,0.9999988$ \\
\hline 3 & 11 & 0.9999984 & $0.9999980,1$ & $0.9999979,0.9999988$ \\
\hline
\end{tabular}

Table 5.3: Conf. Int. using Uniform Epistemic Distributions

\begin{tabular}{|l|l|l|l|l|}
\hline $\begin{array}{l}\text { Num. } \\
\text { Reqd. }\end{array}$ & $\begin{array}{l}\text { Num. } \\
\text { Standby }\end{array}$ & Mean & $\begin{array}{l}95 \% \text { Upper } \\
\text { One-Sided } \\
\text { Conf. Int. }\end{array}$ & $\begin{array}{l}\text { 95\% Two-Sided } \\
\text { Conf. Int. }\end{array}$ \\
\hline 14 & 0 & 0.9992702 & $0.9991657,1$ & $0.9991496,0.9993808$ \\
\hline 13 & 1 & 0.9999978 & $0.9999973,1$ & $0.9999972,0.999998$ \\
\hline 12 & 2 & 0.9999981 & $0.9999975,1$ & $0.9999974,0.9999987$ \\
\hline 9 & 5 & 0.9999981 & $0.9999975,1$ & $0.9999974,0.9999987$ \\
\hline 6 & 8 & 0.9999983 & $0.9999978,1$ & $0.9999977,0.9999987$ \\
\hline 3 & 11 & 0.9999983 & $0.9999978,1$ & $0.9999978,0.9999987$ \\
\hline
\end{tabular}

hardware and software redundancy. Two of the nodes (one on each chassis) are set up to function as proxy for load balancing and to perform failover, while rest of the (six) nodes are application server nodes. Two instances of web application servers (WAS) run on each application server node, sharing the hardware and OS resources. Each WAS instance forms a failover pair (called a replication domain) with WAS installed on another node on a different chassis. In total, there are twelve WAS and hence six replication domains, as shown in Figure 5.4. Failovers are performed by proxy servers. Load-balancing between instances of WAS is performed by an IP sprayer. The availability model does not consider failure-repair behavior of the IP sprayer.

The system has two levels of software implemented fault detection for the application servers : fault detection by workload manager of the cluster (upon detection may trigger failover) and detection by node agent which may result in killing and restarting of application server process or other recovery actions. Upon detection 


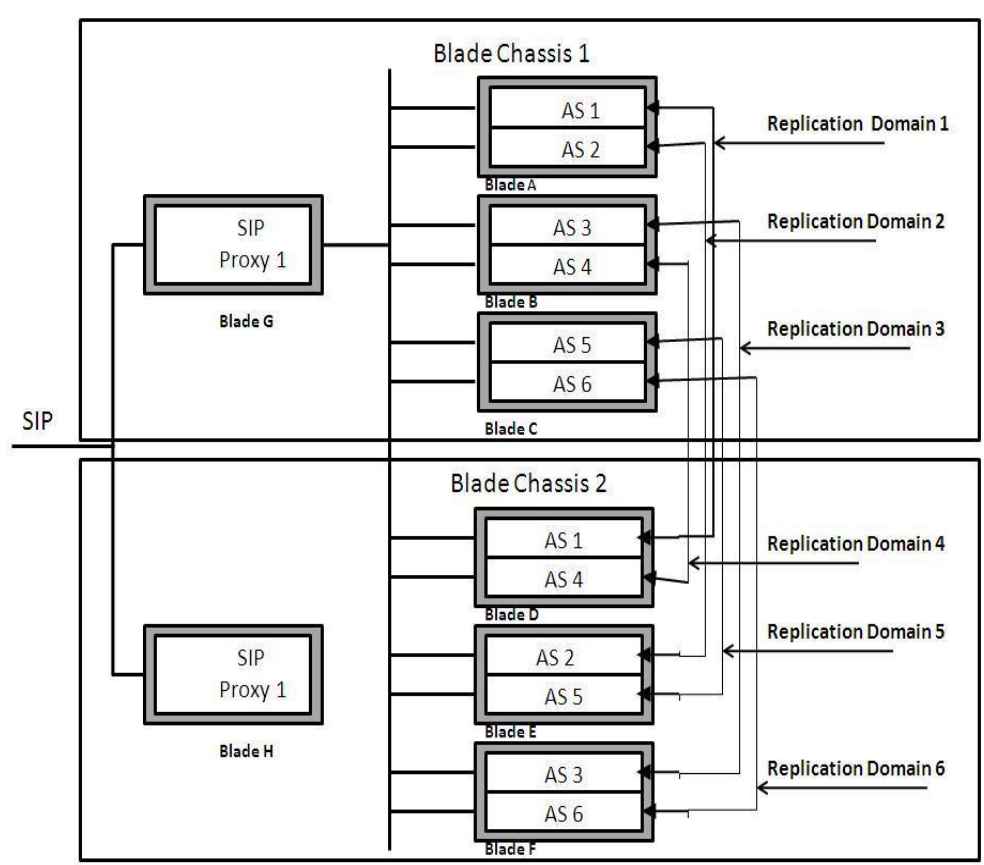

Figure 5.4: IBM SIP SLEE Cluster (adapted from [85])

of failure in the proxy servers or any of the web application servers, a sequence of recovery operations are performed, until recovery is successful. The recovery operations considered in sequence are, automatic process restart, manual process restart, manual reboot and finally manual repair. Subsequent operations are considered only if the previous operation does not lead to successful recovery (escalated levels of recovery).

The availability model for the system comprehensively models the hardware (chassis, CPU, memory, RAID, n/w interfaces etc.) and software (OS, application servers, proxy servers) components of the system and considers different levels of failure detection (manual and two levels of automated software implemented failure detection) and recovery mechanisms. Imperfect coverage is considered both for fault/failure detection as well as for the recovery mechanism. Escalated levels of recovery - failover, process restart, reboot and manual repair are also considered. A 3-level hierarchical model is used where the top two levels are system level and 
sub-system level fault trees while at the bottom level, Markov models are used to model failure-repair behavior of individual hardware or software components. While the entire model is still of considerable size with several subcomponents, hierarchical approach helps limit the state space. The entire hierarchical model has 63 input parameters. To illustrate the different levels and types of sub-models in this hierarchical model, Figure 5.5 shows a part of the hierarchical model. For details on the system and the hierarchical model, the reader is referred to [85].

\section{Hierarchical Composition}

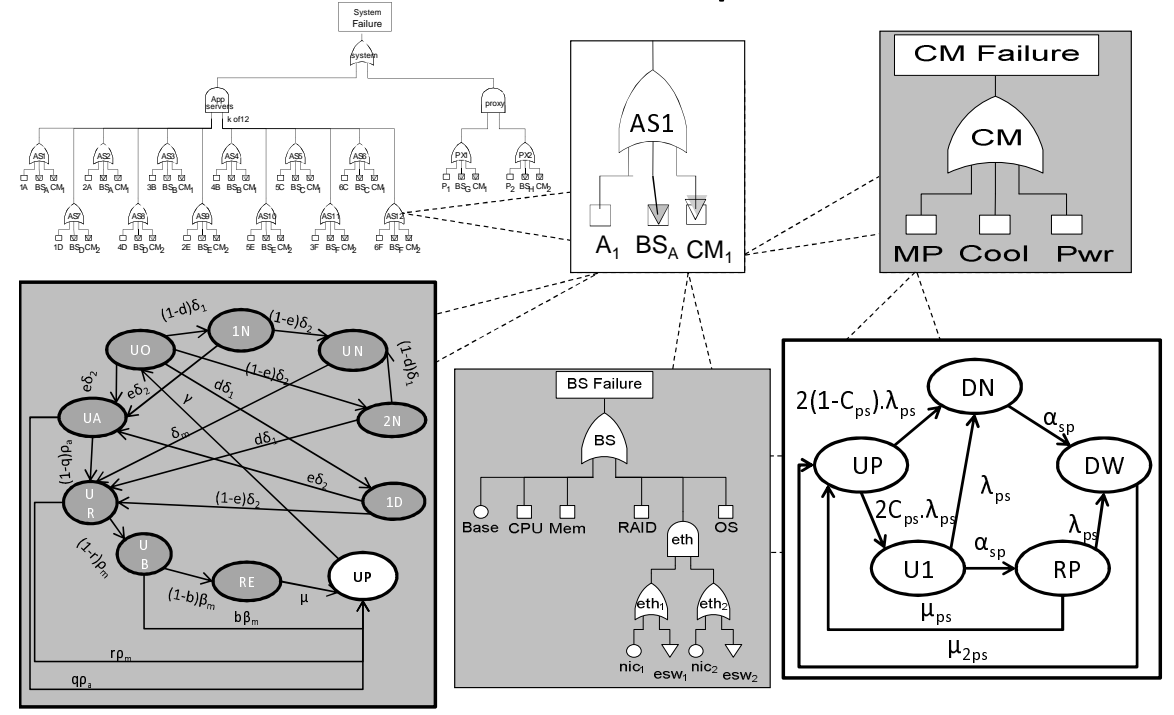

A single monolithic Markov model will have too many states

Figure 5.5: Hierarchical Model of the IBM SIP Server Cluster System

\section{Uncertainty Propagation through the Availability Model}

The point estimates of the model parameters were obtained from Tables 3 and 4 in [85]. As a measure of the epistemic uncertainty in the model parameter values, we use $95 \%$ two-sided confidence intervals for the rate parameters of the model and $95 \%$ upper one-sided confidence interval for the coverage parameters. We choose such 
values for the limits of the $95 \%$ two-sided confidence interval for the input parameter values, so as to get ratio between point estimate and width of confidence interval to be the same as the ratio between the point estimate and the width of the bounds of parameter values as in [82]. The half width of $95 \%$ two-sided confidence interval for parameters for which the bounds have not been specified in [82] are assumed to be at most $10 \%$ of the point estimate. For the coverage parameters, the lower limit of upper one-sided confidence interval is chosen to be at least $90 \%$ of the point estimate.

In order to determine the form and parameters of marginal epistemic distributions of each of the model input parameters, we first determine the number of observations that would have been used to compute the point estimate and $95 \%$ confidence interval, for each of the 63 model input parameters, as explained in Section 5.1.1. The number of observations thus computed, are found to range from 11 to 269 . As explained in Section 5.1.1, the posterior distribution of the failure and repair rates is Erlang and the coverage parameters have a beta posterior distribution.

After determining the marginal epistemic density function for each of the parameters, the total number of samples $n$, to be drawn from each of them, needs to be determined. As explained in Section 5.1.2, the number of samples to be drawn from each of the epistemic distributions, is computed as the largest of the number of observations that would have been used to compute the point and interval estimates, across all the model input parameters. Since, in this example, the number of observations are computed to range from 11 to 269, the number of samples to be drawn from the epistemic distribution of each input parameter is determined to be $n=269$.

We follow the random sampling method to generate random deviates form the epistemic distributions of each of the 63 input parameters . The samples from the Erlang and beta distributions for the parameters are generated using Mathematica's [2] built-in function RandomReal. At each of the $n=269$ sets of values of the param- 
eters, the hierarchical aleatory model is solved using SHARPE [87]. Figure 5.6 shows the empirical CDF of the availability of the SIP server cluster system constructed from the $n=269$ values of model output (availability) obtained by solving the availability model random sampling procedure. Using the $2.5^{\text {th }}$ and $97.5^{\text {th }}$ percentiles

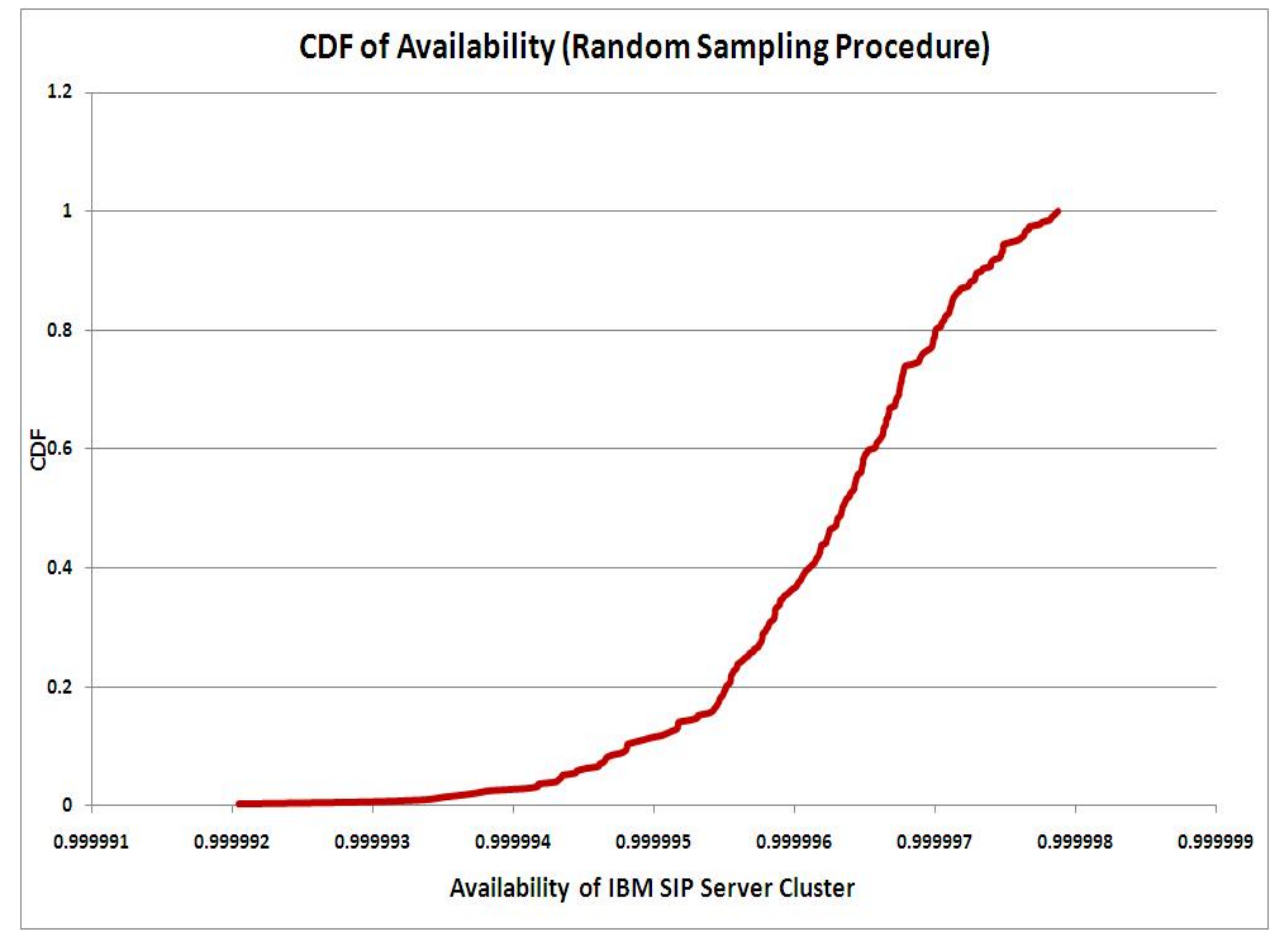

FiguRE 5.6: CDF of SIP server cluster availability

from the CDF in Figure 5.6, the 95\% two-sided confidence interval of availability of IBM SIP SLEE cluster system was computed to be (0.99999433, 0.99999741). Using the $5^{\text {th }}$ percentile, the $95 \%$ upper one-sided confidence interval of availability was obtained as $(0.99999434,1)$. The variance of the availability of the system, due to the epistemic uncertainties in the input parameters of the availability model, was computed to be $9.2147 e-13$. 


\subsubsection{Reliability of Remote Exploration and Experimentation System}

We now apply the sampling based uncertainty propagation method, to the reliability analysis of the NASA Remote Exploration and Experimentation (REE) system $[40,13,90]$. The NASA Jet Propulsion Laboratory REE Project was a large multi-year technology demonstration project to develop a low-power, scalable, faulttolerant, high-performance computing platform for use in space and to demonstrate that significant on-board computing enables a new class of scientific missions. A REE testbed was developed to test, refine, and validate scalability of the architecture and system approaches used to achieve the dependability goals.

The REE system was expected to provide continuous operation through graceful degradation despite experiencing transient or permanent failures. It was expected to experience a small number of radiation induced transient component failures per day. Permanent component failures were expected to be experienced a small number of times over several years. The system provided fault detection and recovery mechanisms so that applications could operate in the presence of faults. Availability and reliability of the REE system was analyzed in [13, 90], with the help of several models, to assess its fault-tolerance features. These models were specified and solved using the SHARPE [87] software package.

The REE system is a collection of processing elements (referred to as nodes) connected together by a Myrinet [1]. Each node is a commercially available computer running commercial off-the-shelf (COTS) operating system based on UNIX. The architecture of REE system is shown in Figure 5.7. Redundancy in hardware, software, time and data are used to achieve fault tolerance. In the version of the REE system analyzed in this chapter, fault detection and recovery are provided by Software Implemented Fault-Tolerance (SIFT) and a System Executive (SE) [22, 68, 69, 70]. The SE is responsible for local error detection and failure recovery while the System 


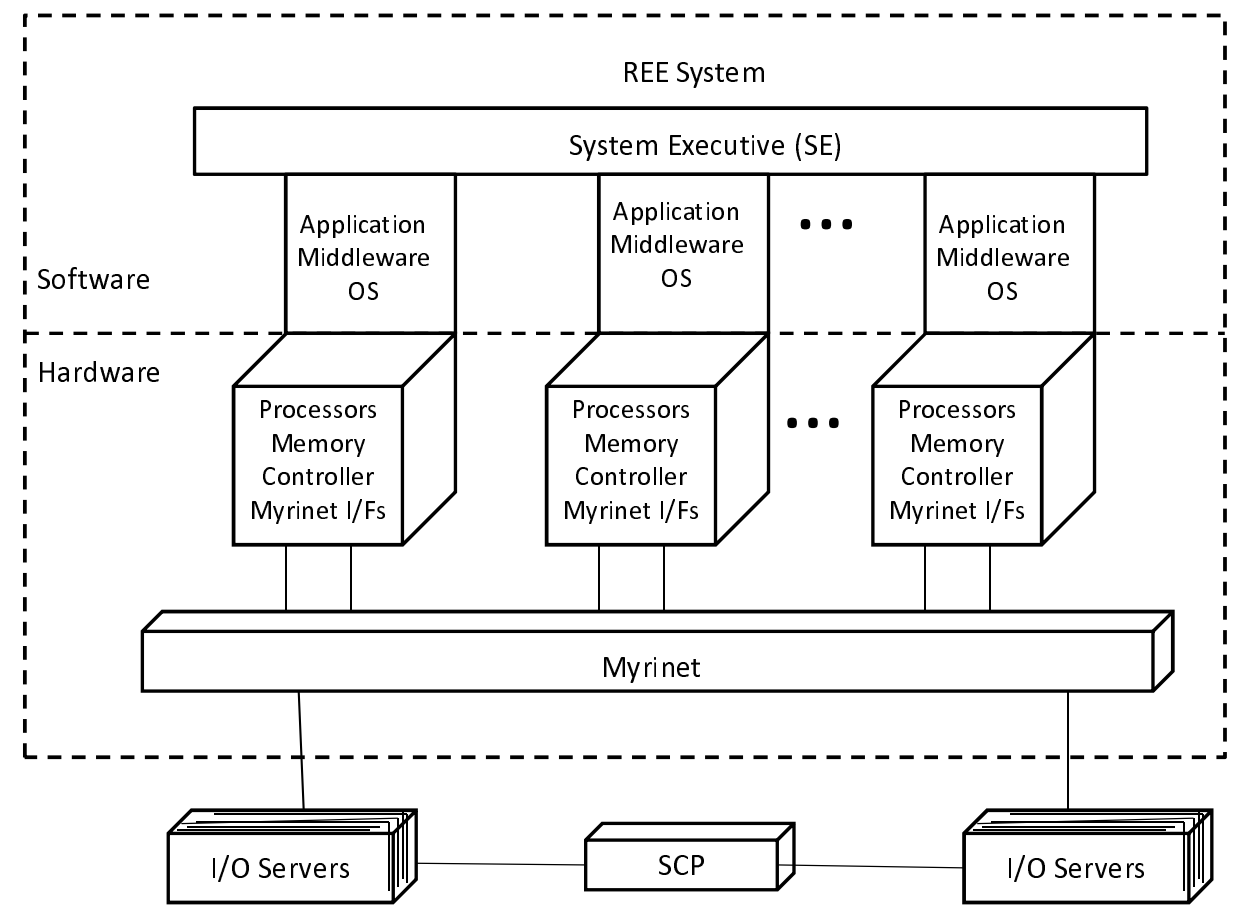

FiguRE 5.7: REE System Architecture (adapted from [13])

Control Processor (SCP) provides fault detection and recovery at the system level. The SCP relies on periodic node health status messages or heartbeats from the SE on each node to determine their status. Nodes can be individually reset/restarted by either the SE or the SCP.

A service request to the system is assigned to multiple nodes to be processed in parallel, by a fault-tolerant scheduler. The same software components have different implementations across different nodes in the system, to provide redundancy and design diversity. Results from these nodes are then compared by the SE to detect and tolerate faults. Intermittent and transient faults [33] can be detected and recovered from, by SIFT, SE or SCP. However, as the REE system operates without human intervention, components cannot be replaced after permanent failures. Hence the permanent failures require the system to be reconfigured to a degraded mode of 


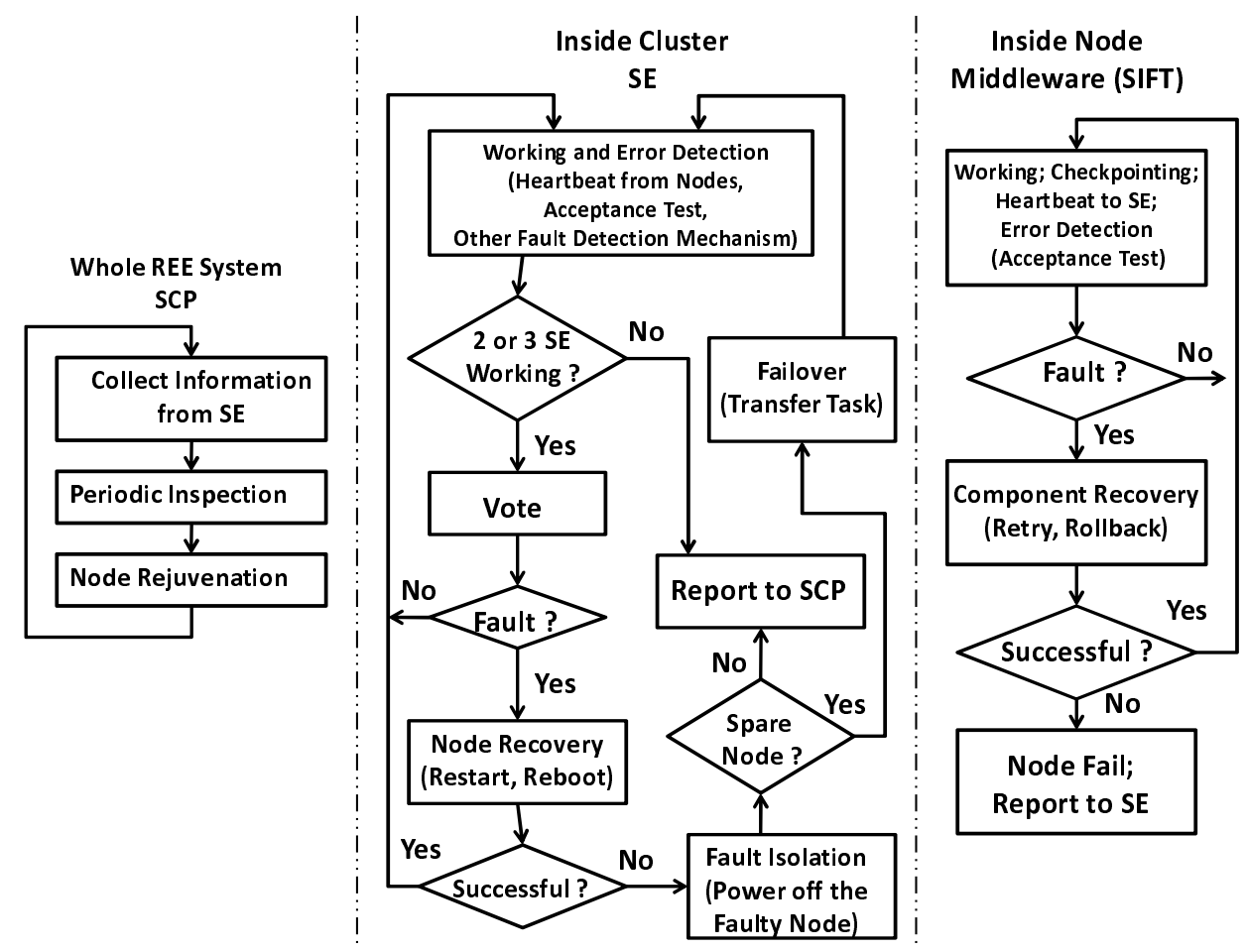

FIGURE 5.8: Flowcharts (adapted from [13])

operation and affect the long term behavior of the system. Figure 5.8 captures the fault detection and recovery scheme employed in this version of the REE computing system.

Hardware and software component failures can be transient or permanent in nature. The majority of transient faults are masked due to the use of error correcting codes and redundant hardware. Unmasked transient failures may freeze the processors making the system unresponsive or generate erroneous outputs. Heartbeat messages are relied upon, by the SE to detect unresponsive or frozen nodes. Erroneous outputs are detected with the help of acceptance tests by SIFT. Upon detection of either an unresponsive node or erroneous output, the node is rebooted after transferring its tasks to other spare nodes (the tasks are restarted from the last checkpoint). In addition, the SCP selectively reboots several nodes as a preventive 
action, according to their uptime and current health status. This preventive action, which is called rejuvenation, helps in getting rid of latent faults and effectively tolerate "soft" or aging related faults such as memory leaks [26].

Transient and intermittent faults are assumed to not affect reliability of the system as after recovery actions, the failed nodes will eventually return to use. If there is a permanent fault involved, the faulty node cannot be rebooted successfully. In this case, a notification is sent to the SE, the faulty node will be isolated, and the system reconfigured with the remaining working nodes. Eventually, when the number of working nodes is smaller than the minimum requirement ( 2 in the testbed), the whole system fails.

We use the reliability model of the REE system as an example to illustrate the uncertainty propagation method. Hence we will only consider permanent faults.

\section{Reliability Model of REE System}

A two-stage hierarchical model [87] is used to capture the failure behavior of the system and its components in [13]. Here, we modify the model to make it a threestage hierarchical model to capture the failure behavior of the software components in greater details. At the lowest level, a Markov model captures the failure behavior of the software subsystem. In the middle level, the failure behavior of individual components is captured using reliability block diagrams (RBD). The reliability computed from the Markov model, at the lowest level, provides one of the inputs to the RBD. The top level model is a fault tree that takes the reliability of each of the individual component (obtained by solving the middle level RBD model), as an input and takes into account the interactions between the individual components to provide the overall system reliability. The modified three-stage hierarchical model is shown in Figure 5.9.

The REE architecture uses redundancy at several levels. Each node uses redun- 


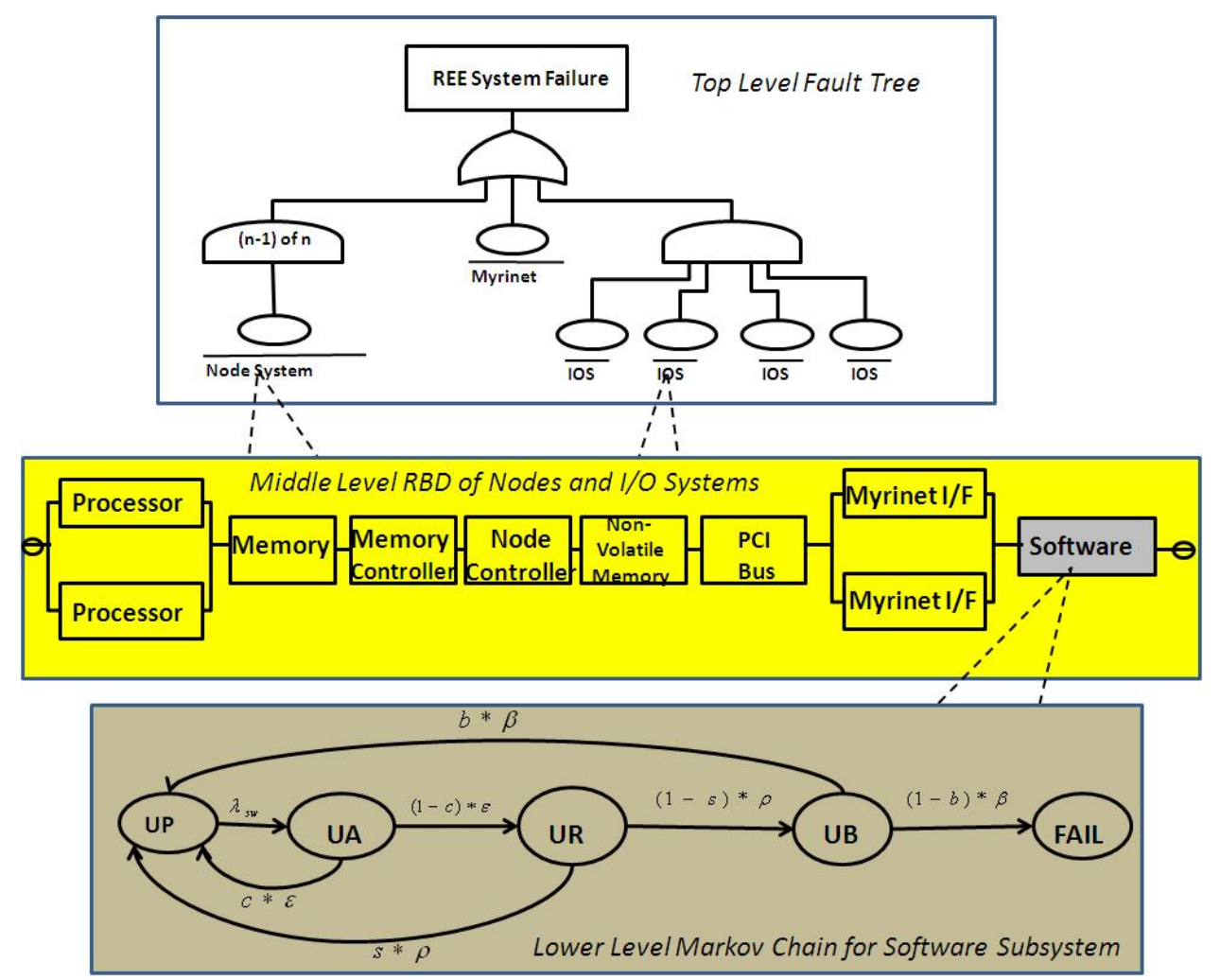

Figure 5.9: Hierarchical Reliability Model of the REE System

dancy in the form of spare processor chips and spare Myrinet interfaces to increase its reliability. The node subsystem is configured as a k-out-of-n system of such nodes (with $k=2$ in the prototype implementation). The I/O subsystem also has redundancy in its configuration and is implemented as a parallel system with several redundant nodes. All the nodes in node system as well as the I/O system are assumed to exhibit the same stochastic behavior regarding faults. The faults are considered to be mutually independent. Since the REE system operates without human intervention, no replacement of components after a permanent failure has been assumed. It is these permanent failures of components that are considered in the reliability model. Most software failures in the nodes are expected to be recovered from by retry, process restart or node reboot. The Markov model of the software subsystem shown in Figure 5.9 and reproduced in Figure 5.10 for clearer illustration, captures 
these escalated levels of recovery actions. The software component is considered to have failed only if the software failures cannot be recovered from, even after node reboot. Software failures that cannot be recovered from even after retry, restart or reboot can be identified with Bohrbugs while those that can be recovered from by use of these actions, can be identified with Mandelbugs [26, 25]. Various rates and

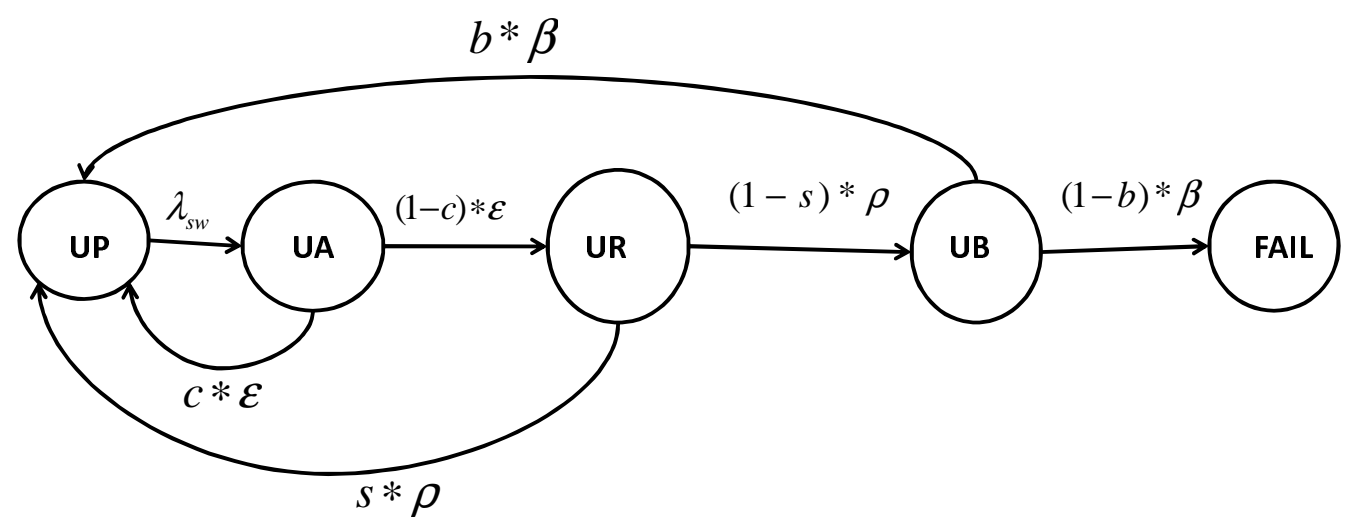

FiguRE 5.10: Markov model for Reliability of Software

coverage probabilities in Figure 5.9 have been defined in the nomenclature table. Rates of failure, retry, restart and reboot as well as the coverage probabilities in the Markov model in Figure 5.10 have also been defined in the nomenclature table.

Uncertainty Propagation through the reliability model

The times to failure of individual components in the middle level RBD model in Figure 5.9 are assumed to follow an exponential distribution. Table 5.4 summarizes the point estimates of the parameters of the time to failure distribution of various components in the REE system and coverage probabilities of various recovery actions (in Figure 5.10). To represent the uncertainty in the model input parameters, we choose the half-width of $95 \%$ two-sided confidence interval of the rate parameters to range from $15 \%$ to $33 \%$ of the point estimate. For the coverage parameters, we 
choose the lower limit of the $95 \%$ upper one-sided confidence interval to be ranging from $80 \%$ to $90 \%$ of the point estimate.

Table 5.4: Point Estimates of Reliability Model Parameters

\begin{tabular}{|c|c|}
\hline Parameter & Value \\
\hline$\lambda_{\text {pro }}$ & 0.01 \\
\hline$\lambda_{\text {mem }}$ & 0.03 \\
\hline$\lambda_{\text {bmc }}$ & 0.01 \\
\hline$\lambda_{\text {ndc }}$ & 0.01 \\
\hline$\lambda_{\text {nvm }}$ & 0.01 \\
\hline$\lambda_{\text {bus }}$ & 0.02 \\
\hline$\lambda_{\text {nif }}$ & 0.03 \\
\hline$\lambda_{\text {net }}$ & 0.0006 \\
\hline$\lambda_{\text {sw }}$ & 8.76 \\
\hline$\varepsilon$ & 31536000 \\
\hline$\rho$ & 3153600 \\
\hline$\beta$ & 52560 \\
\hline$c$ & 0.9 \\
\hline$r$ & 0.9 \\
\hline$b$ & 0.9 \\
\hline
\end{tabular}

The steps in uncertainty propagation through the reliability model of the REE system are as follows :

(a) Determining the Epistemic Distribution of Model Parameters: We first determine the number of observations that would have been used to compute the point estimate and 95\% confidence interval, for each model parameter, as explained in Section 5.1.1. The number of observations, thus calculated for each model input parameter, is summarized in Table 5.5. Since the times to failures of the components in the system have been assumed to follow exponential distribution, as explained in Section 5.1.1, the epistemic distributions of the parameters as random variables are calculated to be Erlang distribution with parameters as summarized in Table 5.5. The parameters of the beta epistemic distributions (of the coverage parameters as 
random variables) are also provided in the table.

Table 5.5: Epistemic Distributions of Parameters

\begin{tabular}{|c|c|c|}
\hline Parameter & Num. Observations & Epistemic Distributions \\
\hline$\lambda_{\text {pro }}$ & 117 & $\operatorname{Erlang}(117,8.57 \mathrm{e}-5)$ \\
\hline$\lambda_{m e m}$ & 167 & $\operatorname{Erlang}(167,1.79 \mathrm{e}-4)$ \\
\hline$\lambda_{b m c}$ & 92 & $\operatorname{Erlang}(92,1.08 \mathrm{e}-4)$ \\
\hline$\lambda_{n d c}$ & 117 & $\operatorname{Erlang}(117,8.54 \mathrm{e}-5)$ \\
\hline$\lambda_{n v m}$ & 103 & $\operatorname{Erlang}(103,9.71 \mathrm{e}-5)$ \\
\hline$\lambda_{b u s}$ & 146 & $\operatorname{Erlang}(146,1.37 \mathrm{e}-4)$ \\
\hline$\lambda_{n i f}$ & 208 & $\operatorname{Erlang}(208,1.44 \mathrm{e}-4)$ \\
\hline$\lambda_{n e t}$ & 19 & $\operatorname{Erlang}(19,4.21 \mathrm{e}-5)$ \\
\hline$\lambda_{s w}$ & 65 & $\operatorname{Erlang}(81,1.54 \mathrm{e}-3)$ \\
\hline$\beta$ & 81 & $\operatorname{beta}(52,5)$ \\
\hline$b$ & faults injected $: 57$, faults detected 51 & \\
\hline
\end{tabular}

(b) Determining Number of Samples from Epistemic Distributions:

The number of samples needed from the epistemic distribution of each input parameter is determined to be $n=208$, largest of all the number of observations of the input parameters.

(c) Sampling Procedure: To implement the random sampling procedure, we use Mathematica's [2] built-in function RandomReal. We generate $n=208$ random deviates from the epistemic distributions of each of the input parameters.

(d) Solving the Analytic Model: At each of the $n=208$ set of values of the parameters, the hierarchical model is solved using SHARPE [87].

(e) Summarizing the Output Values: The empirical Cumulative Distribution Function $(\mathrm{CDF})$ of the reliability of the system output is constructed from the $n=$ 208 values of the output obtained by solving the system availability model using SHARPE. Figure 5.11 shows the empirical CDF of the reliability of the REE system at a time $t=5$ years.

The $5^{\text {th }}$ percentile from the CDF provides the lower limit for the $95 \%$ upper one-sided confidence interval of reliability, which is computed to be $(0.955861,1)$. 


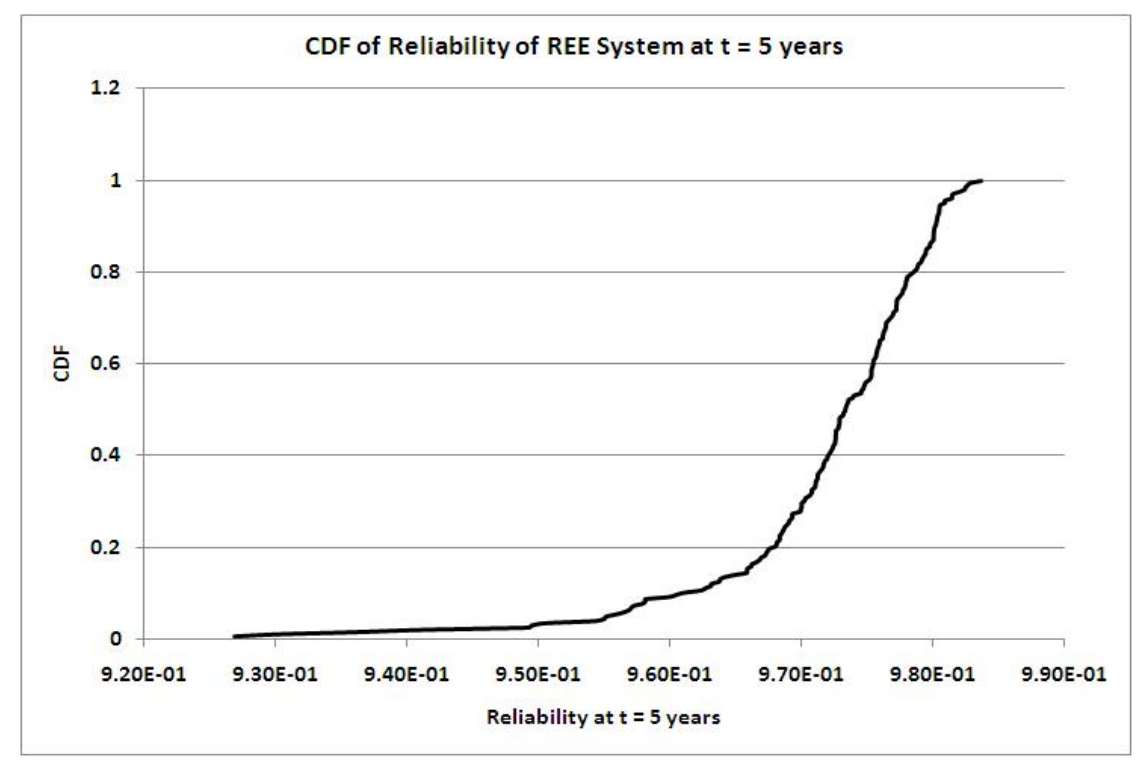

Figure 5.11: CDF of Reliability of REE System at $t=5$ years

The upper and lower limits of the two-sided 95\% confidence interval of reliability are provided by the $97.5^{\text {th }}$ percentile and $2.5^{\text {th }}$ percentile, respectively. The two-sided $95 \%$ confidence interval of reliability is computed to be $(0.949485,0.981994)$.

\subsubsection{Mean Response Time and Reliability of Composite Web Service}

As another example to illustrate the versatility and the ease of use of this uncertainty propagation method, we perform uncertainty propagation through the service reliability and performance model of a composite web service called Travel-Agent, which was discussed in [75].

A composite web service combines several different web services to provide a new service to its users. It uses high level languages such as BPEL (Business Process Execution Language) [3] to quickly combine the existing web services into new services. The web service Travel-Agent, interacts with different services to make airlines and hotel reservations for customers. Each of the individual interactions (web services) may have complex interactions within themselves and have their own mean response time and reliability. When initialized, it first looks up for vacancy, fare and other 
information from two different airlines, in parallel. Upon getting the response from both, it chooses one of them, based on some requirements (e.g., fare, schedule, etc.). If only one of them responds, it chooses the only response. Then it continues to make airlines reservation for the chosen airlines. After making airline reservation, it makes hotel reservation and notifies the user. Each of these operations may fail and can be retried/restarted. The entire process is considered successful only if a successful airline and hotel reservation is made and a reply is sent back to the user. The look up for vacancy in airlines happens concurrently on both airlines. While the details of the service can be obtained from [75], the BPEL representation of the service, has been reproduced in Figure 5.12, for ease of reference. A CTMC model is

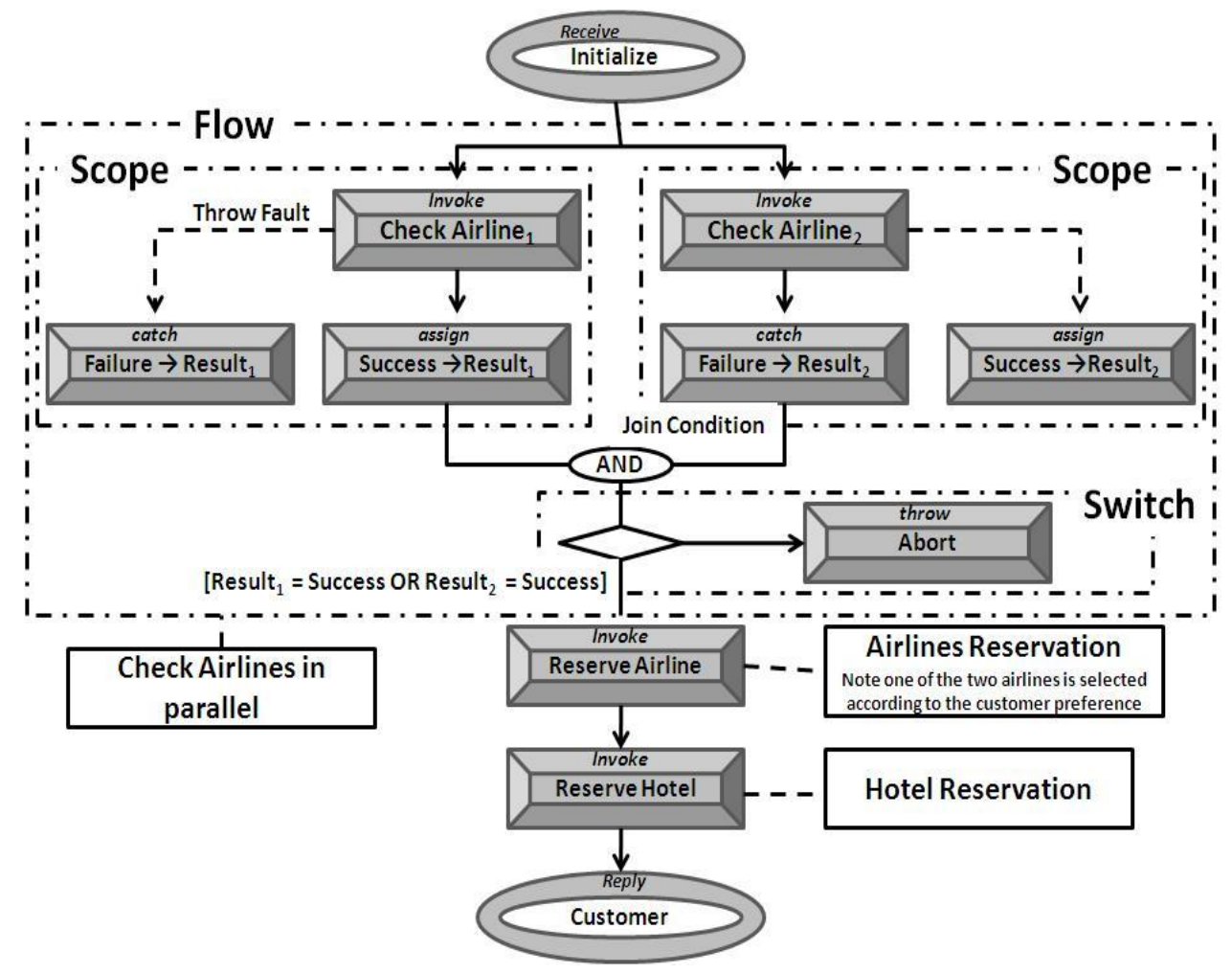

Figure 5.12: BPEL representation of Travel-Agent Process

formulated to study the overall reliability and response time of the entire composite web service. The response time of each of these services is assumed to follow an ex- 
ponential distribution. The reliability of each of the individual services is computed as the ratio of number of successful executions of the service to the total number of executions of the service attempted and so it can be considered to be parameter of Bernoulli aleatory distribution. The CTMC considers concurrent execution of the two airlines services, failure of any of the services and restarts/retries of services to attempt recovery from failure of a service. It also considers overhead of time spent in restarts (assuming this overhead time to follow an exponential distribution) as well as imperfect coverage of restarts of services. While the details of the CTMC model can be seen at [75], it has been reproduced in Figure 5.13, for ease of reference. In

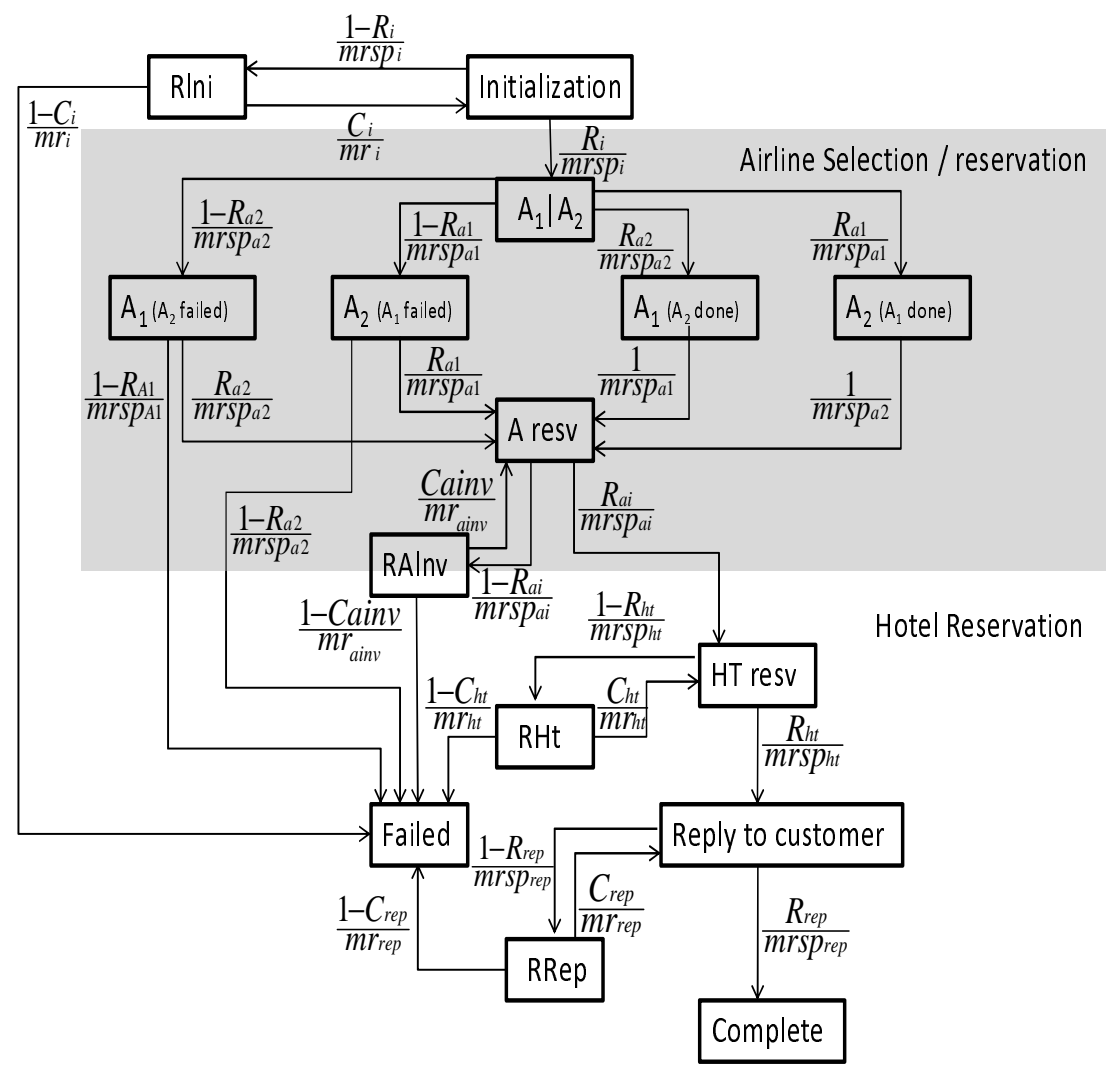

Figure 5.13: CTMC Model of Composite Web Service

the CTMC in Figure 5.13, the "Initialization" has mean response time of $m r s p_{i}$ and 
reliability $R_{i}$. Upon failure of this state, it is restarted with mean overhead time $m r_{i}$ (from state $R I n i$ ). The restart can be successful with a coverage parameter $C_{i}$. The concurrent airline selection process has been broken down into 5 states. " $A_{1} \mid A_{2}$ " depicts the state when both airlines selection processes are ongoing. Rest of the four states depict one of the airlines selection process ongoing while the other has either finished or failed. Retry is not considered for airlines selection process. The mean response times for airline selection process are $m r s p_{a 1}$ and $m r s p_{a 2}$ and reliabilities are $R_{a 1}$ and $R_{a 2}$, for airlines 1 and 2 , respectively. Restart and imperfect coverage of restart is considered for the states airline reservation "A resv", hotel reservation "HT resv" and "Reply to customer".

\section{Uncertainty Propagation through service reliability and performance model}

The point estimates of the model parameters for computation of mean service response time and overall service reliability, were obtained from Tables 1 and 2, respectively, in [75]. To represent the uncertainty in the model input parameters, the confidence intervals of input parameters are chosen as: half-width of $95 \%$ two-sided confidence interval of rate parameters to be at most $10 \%$ of point estimate and lower limit of $95 \%$ upper one-sided confidence interval to be at least $90 \%$ of point estimate. The aleatory distribution for rate parameters (reciprocal of mean response times for each process and the mean overhead for restart of each process) is considered to be exponential, while aleatory distribution for coverage parameters and reliability of each process is considered to be Bernoulli distribution. The number of observations (or number of attempted executions of the services) that would have been used to compute the point estimate and the given confidence intervals of the parameters, are computed, as explained in Section 5.1.1. The number of observations (or number of attempted executions of services), thus calculated, for parameters used in response time computation of the composite web service, range from 67 to 421, for the param- 
eters used in overall reliability computation, they range from 67 to 123 . The form and parameters of epistemic distribution of each parameter are derived next: Erlang for the mean service response time parameters and beta for the reliability as well coverage parameters, as explained in Section 5.1.1.

As explained in Section 5.1.2, the number of samples to be drawn from each of the epistemic distributions, is computed as the largest of the number of observations that would have been used to compute the point and interval estimates, across all the model input parameters. Since, the number of observations (or number of attempted executions of services), for parameters used in response time computation of the composite web service, range from 67 to 421, the number of samples to be drawn from the epistemic distribution of each input parameter is determined to be $n=421$. The number of observations computed for the parameters used in overall reliability computation range from 67 to 123 and hence the number of samples to be drawn from the epistemic distribution of each input parameter is determined to be $n=123$, for this example.

We follow the random sampling procedure as explained in Section 5.1.3. The samples from the Erlang and beta distributions for the parameters are generated using Mathematica's [2] built-in function RandomReal, in the same manner as discussed in the previous examples. However in this case, the CTMC models can be solved to obtain closed-form solutions for both the mean service response time as well as the overall service reliability. Therefore, at each of the $n$ sets of values of the parameters, we evaluate the closed-form expressions using Mathematica (unlike using SHARPE to solve the model as in the previous examples in this chapters in Sections 5.2.1, 5.2.2 and 5.2.3, where closed-form solutions did not exist).

Figure 5.14 shows the empirical CDF of the mean service response time, taking into account the restarts of processes and overhead of time spent in retries, constructed from the $n=421$ values of output obtained by the LHS sampling pro- 


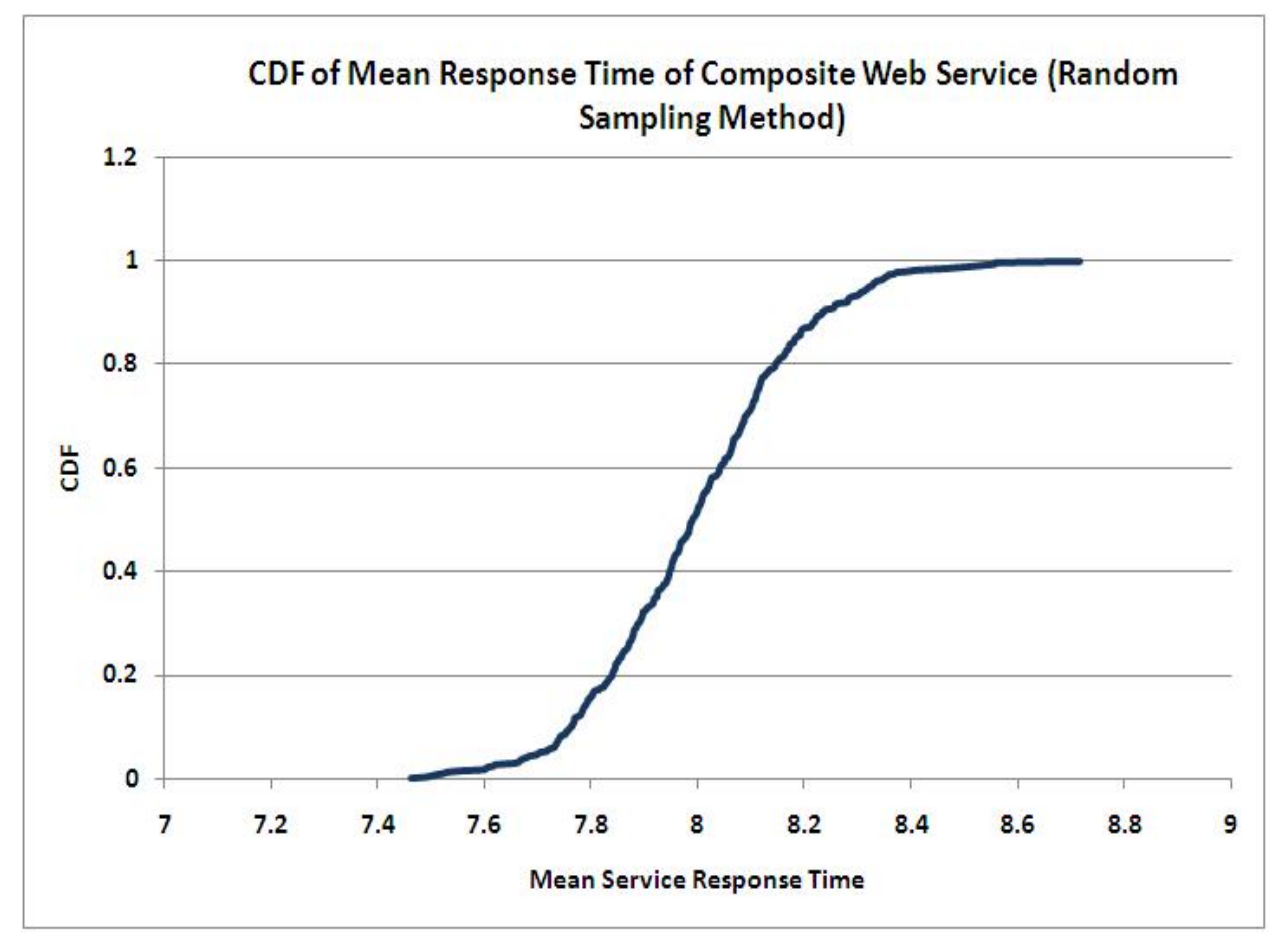

Figure 5.14: CDF of Mean Service Response Time (Random Sampling)

cedure. Based on this CDF, the $95 \%$ two-sided confidence interval of mean service response time of the composite web service is calculated to be $(7.61841,8.36969)$. the variance of the mean response time of the composite web service, due to the epistemic uncertainties in the input parameters of its aleatory model is computed to be 0.0369908 .

The closed-form expression for overall reliability of the composite web service, $R_{\text {sys }}$, upon considering perfect coverages for airline reservation (not airline selection) and hotel reservation and perfect reliability for customer reply and service initialization (parameter values as considered for the reliability computation in [75]), reduces to:

$$
R_{\text {sys }}=R_{a 1}+R_{a 2}-R_{a 1} \cdot R_{a 2}
$$

The epistemic density function for $R_{a 1}$ and $R_{a 2}$ are determined to be beta distribution with parameters 99 and 26. Figure 5.15 shows the empirical CDF of the service 
reliability, obtained by using the random sampling procedure.

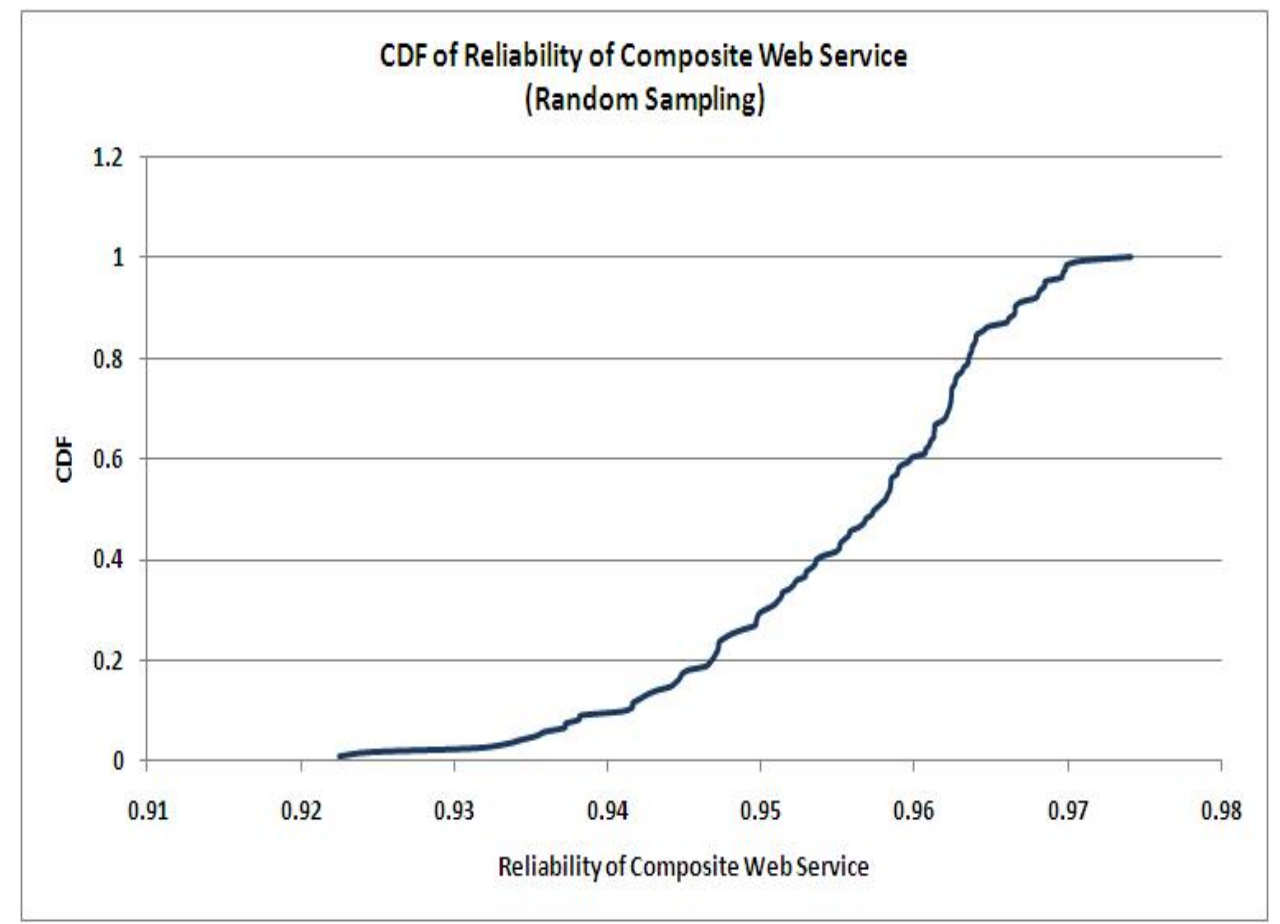

Figure 5.15: CDF of Reliability of Composite Web Service

The $95 \%$ two-sided confidence interval of reliability of the composite web service (without considering restarts) is calculated to be $(0.931374,0.969912)$ and the variance of reliability of composite web service due to epistemic uncertainties in the reliability model inputs is calculated to be 0.000107 . 


\section{6 Latin Hypercube Sampling}

The random sampling based epistemic uncertainty propagation discussed in Chapter 5 can be applied to a wide range of models in a non-intrusive way and is easy to understand and implement. However, since it draws sample elements randomly from the entire sample space, it does not guarantee that sample elements will be generated from any particular subset of sample space. Therefore, low probability yet high impact regions of the sample space may be missed or a large number of sample elements may be needed to cover them. Stratified sampling addresses this by dividing the entire sample space into regions or strata, each with a probability of sampling (strata probability). Depending on the strata probability, it results in more samples to be obtained from one subset of the sample space over another. However, to implement it, one has to define the strata and strata probabilities, which is significant task in itself. Latin Hypercube Sampling (LHS) provides properties between random sampling and stratified sampling. Like random sampling, it is easy to implement and does not require defining the strata and its probabilities, yet similar to stratified sampling, it is more likely to reach even low probability regions of the sample space. It can be thought of as stratified sampling with equal strata size and one sample 
from each stratum [30]. It is known that for a given sample size, a statistic of model output obtained by LHS sampling, will have a variance less than or equal to that obtained by random sampling. In other words, at a particular sample size, LHS sampling yields a more efficient estimator for a statistic of model output than that by random sampling, providing a more robust sampling procedure [29, 50]. It can be seen later in Chapter 7 that LHS sampling also enables relaxing the assumption of epistemic independence between the input parameters of the aleatory model.

In this chapter, we discuss epistemic uncertainty propagation through stochastic dependability models, using Latin Hypercube Sampling. We first introduce the method and then illustrate it with the help of dependability or performance models of several real life systems. We also show that the mean of aleatory model output has lesser variance when using LHS as compared to that obtained using random sampling and hence LHS provides a more robust sampling method in these examples ${ }^{1}$.

The aleatory models considered are of varying complexity (simpler Markov chain model to large hierarchical model with 63 input parameters) and have a wide range of output metrics (availability of large systems, reliability, mean response time, capacity oriented availability, handoff call arrival rate). While the uncertainty propagation wrapper has been implemented in Mathematica, the models themselves are solved in SHARPE, SPNP or Mathematica [2, 87, 32].

\subsection{Uncertainty Propagation with LHS}

Most of the steps in epistemic uncertainty propagation through stochastic dependability models, using Latin Hypercube Sampling are the same as those with random sampling, as explained in Section 5.1, except the sampling step.

We first derive the epistemic distribution of each of the $k$ model input parameters (marginal distribution for each parameter, assuming epistemic independence). Then

${ }^{1}$ Adapted from [55, 54, 52, 53] 
we determine the number of samples, $n$ to be drawn from each of these epistemic distributions, assuming the input parameters to be independent. Subsequent to the determination of epistemic distribution of each input parameter, $n$ samples or random deviates need to be drawn from each of the $k$ epistemic distributions.

We employ Latin Hypercube Sampling (LHS) procedure in our uncertainty propagation method. Latin Hypercube Sampling method (LHS) divides the entire probability space into equal intervals (number of intervals is equal to the number of samples needed) and draws a random sample from each of the intervals. Thus it is almost guaranteed to address the entire probability space evenly and easily reaches low probability - high impact areas (rare events) of the epistemic distribution of input parameters. Once the samples are generated, random pairings without replacement is carried out between samples of different parameters, to ensure randomness in sequence of the samples from distributions of different parameters. The actual samples of random numbers or the random deviates can be generated by any of the methods like inverse transform method, rejection sampling, Box-Mueller transform or Johnson's translation [37].

The range of each parameter as random variable $\Theta_{i}(i=1,2, \ldots, k)$, is divided into $n$ equal intervals (disjoint and exhaustive). One random sample is drawn from each of the $n$ intervals. $n$ samples drawn from each distribution, result in an $n-b y-k$ matrix. The elements of each column in this matrix are then randomly rearranged. This rearrangement is necessary to ensure randomness in order of samples obtained by LHS sampling procedure. Each of the $n$ rows of the matrix $\boldsymbol{V}$, after rearrangement, can be considered as $n$ vectors $\left(\boldsymbol{v}_{\mathbf{1}}\right.$ through $\left.\boldsymbol{v}_{\boldsymbol{n}}\right)$ of model input parameter values as:

$$
\boldsymbol{V}=\left[\begin{array}{c}
\boldsymbol{v}_{\mathbf{1}} \\
\boldsymbol{v}_{\mathbf{2}} \\
\vdots \\
\boldsymbol{v}_{\boldsymbol{n}}
\end{array}\right]=\left[\begin{array}{c}
\theta_{11}, \theta_{21}, \ldots \theta_{k 1} \\
\theta_{12}, \theta_{22}, \ldots \theta_{k 2} \\
\vdots \\
\theta_{1 n}, \theta_{2 n}, \ldots \theta_{k n}
\end{array}\right]
$$


The aleatory model is solved at each of the $n$ vectors of input parameter values obtained from their epistemic distributions. Solving the model at each of these sets of input parameter values, yields a set of $n$ values of the model output metric, $M_{1}$ through $M_{n}$. An empirical CDF is constructed out of these $n$ model output values. The values of model output at appropriate percentile points from the empirical CDF, provide the confidence interval of model output.

In the remainder of this chapter, we illustrate epistemic uncertainty propagation using LHS, with the help of dependability or performance models of some real life systems. We also show that LHS provides a more robust sampling method than random sampling, in these examples.

\subsection{Illustrative Examples}

In this section, we illustrate epistemic uncertainty propagation through dependability models, using Latin Hypercube Sampling method, with the help of several examples with a wide range of model types, output metrics and model complexity. These examples include availability model of IBM SIP SLEE server cluster system, reliability of REE system, reliability and mean response time model of a composite web service, performability model of a cable modem termination system (with capacity oriented availability as output metric) to the performance model of a cellular wireless communication network cell (with handoff call arrival rate as output metric). The model types range from simple CTMC models with few parameters to large hierarchical model with 63 input parameters and even a fixed-point iteration model. While the uncertainty propagation wrapper for all these examples is implemented in Mathematica, the aleatory models themselves are solved using SHARPE, SPNP and Mathematica $[87,32,2]$, depending on the model type. We do not manipulate the aleatory model or its output and use the uncertainty propagation wrapper around the pre-existing model solution method. 
These examples illustrate the versatility and wide applicability of sampling based epistemic uncertainty propagation through stochastic dependability models of computer and communication systems, using Latin Hypercube Sampling. They also demonstrate that LHS provides a more robust sampling method than random sampling, in these examples.

Since most of the steps in epistemic uncertainty propagation using Latin Hypercube Sampling method are same as the steps in epistemic uncertainty propagation via random sampling, only the sampling step and the results are shown here for examples that are used to illustrate both these methods. For details not presented in this section, the reader is referred to Section 5.2.

\subsubsection{Availability of IBM SIP Server Cluster System}

As the first example to illustrate sampling based epistemic uncertainty propagation, using Latin Hypercube Sampling, we use the availability of IBM SIP SLEE server cluster, discussed in [85]. Uncertainty propagation through this hierarchical aleatory model, by the random sampling method, has already been presented in Section 5.2.2. Since the steps of determining the epistemic uncertainty and the number of samples $n$ from each epistemic distribution are the same as in Section 5.2.2, we skip the details of these two steps here.

The number of samples needed from the epistemic distribution of each of the 63 input parameters is determined to be $n=269$, largest of all the number of observations of the input parameters. We follow the Latin Hypercube Sampling procedure as explained in Section 6.1. To implement the LHS procedure, we first divide the probability space into $n=269$ intervals and then use Mathematica's built-in functions RandomReal and Quantile to generate the random deviates from different intervals in the distribution. We then use the function RandomSample to obtain random pairing without replacement, between samples from different epistemic distributions. At 
each of the $n=269$ sets of values of the parameters, the hierarchical aleatory model is solved using SHARPE [87]. Figure 6.1 shows the empirical CDF of the availability of the SIP server cluster system constructed from the $n=269$ values of output obtained by LHS procedure.

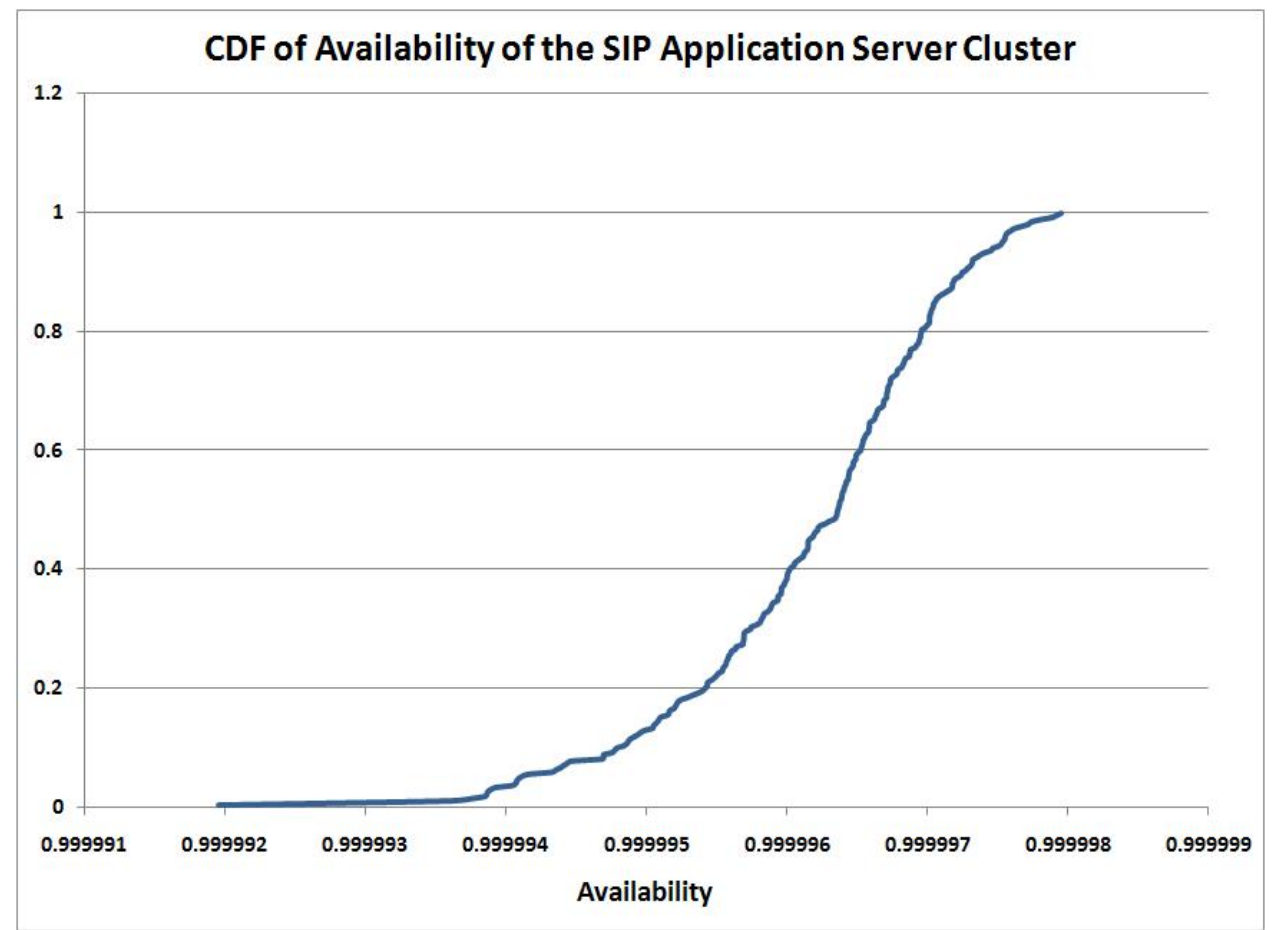

Figure 6.1: CDF of SIP Server Cluster Availability

Using the $2.5^{\text {th }}$ and $97.5^{\text {th }}$ percentiles from the CDF in Figure 6.1, the $95 \%$ two-sided confidence interval of availability of IBM SIP SLEE cluster system was computed to be $(0.99999509,0.99999649)$. Using the $5^{\text {th }}$ percentile, the $95 \%$ upper one-sided confidence interval of availability was obtained as $(0.99999533,1)$. We next evaluated the robustness of the LHS sampling as compared to the random sampling procedure. We made 20 runs of the entire uncertainty propagation method, each run with 269 samples from each epistemic distribution, using both the LHS sampling as well as random sampling. The variance of sample mean of the availability, computed in each of the 20 runs was computed as an indicator of the robustness of the sampling 
procedures. Figure 6.2 shows the CDF of sample mean of availability of the IBM SIP cluster system. The variance obtained by using LHS sampling was $1.12264 E-16$, while that computed by using random sampling was $2.78972 E-15$. Thus in this example, LHS provides a more robust sampling method.

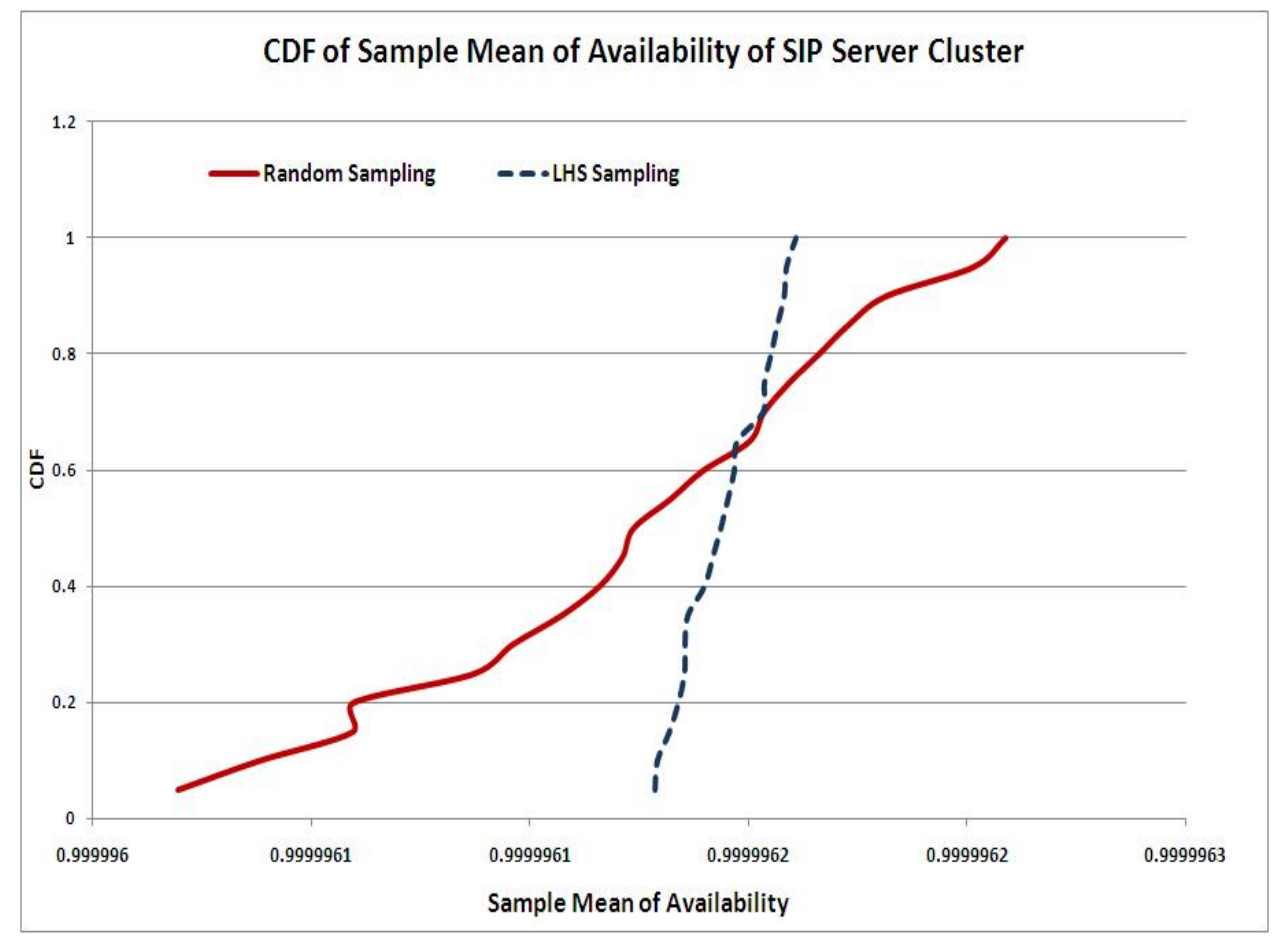

Figure 6.2: CDF of Sample Mean of SIP Server Cluster Availability

Next we compare the time taken to perform the epistemic uncertainty propagation through the hierarchical availability model of the IBM SIP server cluster system by using LHS method, to that taken when using the random sampling procedure. The time taken was determined using AbsoluteTiming function of Mathematica [2], which provides the real (wall clock) time, ignoring any time taken in printing or formatting of the outputs. The timings were measured on a system with dual-core Intel i5 processor with clock frequency of 2.40 Ghz. and 2.92GB of RAM, running Microsft Windows XP Professional, operating system. At the sample number of samples, the difference in time taken between the two sampling procedures can be 
attributed to the extra steps in LHS for dividing the probability space and performing random pairings without replacement between samples from different epistemic distributions (the number of executions of the aleatory model and the number of samples from each epistemic distribution being the same in both cases). However, since LHS requires fewer samples to provide a more robust estimate and cover the entire probability space, the computational savings in drawing fewer samples from the epistemic distributions and fewer executions of the aleatory model can more the compensate for the computational time taken to divide the probability space and perform random pairings without replacement between samples from different epistemic distributions. For epistemic uncertainty propagation through the hierarchical availability model of the IBM SIP server cluster system, the time taken by LHS method, measured using the AbsoluteTiming function of Mathematica [2], on a was 118.14 seconds, while that by the random sampling method was 95.86 seconds.

\subsubsection{Reliability of Remote Exploration and Experimentation System}

As the next example, we choose epistemic uncertainty propagation through the reliability model of the NASA Remote Exploration and Experimentation system, discussed in $[40,13,90]$. The example system and its modified 3-level hierarchical reliability model has been introduced in Section 5.2.3. Propagation of epistemic uncertainty in the parameters of the aleatory hierarchical model, via random sampling method, has also been presented in Section 5.2.3. Therefore, in this section we skip the steps of determination of form and parameters of epistemic distributions and determination of number of samples from each epistemic distribution (steps common to both random sampling and LHS based epistemic uncertainty propagation).

The type and parameters of epistemic distribution of each of the aleatory model input parameter as random variable, has been summarized in Table 5.5. The number of samples $n$, to be drawn from each of the epistemic distributions is calcualted to 
be $n=208$, largest of all the number of observations of the input parameters.

Sampling Procedure: To implement the LHS procedure, we use Mathematica's built-in function RandomReal to generate random deviates from Uniform distribution in the intervals the probability space has been divided into, for LHS sampling. Using those random deviates, we obtain quantiles from Erlang or beta distribution using Mathematica's function Quantile. We also use the function RandomSample to obtain random pairing without replacement, between samples from different epistemic distributions.

Solving the Analytic Model: At each of the $n=208$ set of values of the parameters, the hierarchical model is solved using SHARPE [87].

Summarizing the Output Values: The empirical Cumulative Distribution Function (CDF) of the reliability of the system output is constructed from the $n=$ 208 values of the output obtained by solving the system availability model using SHARPE. Figure 6.3 shows the empirical CDF of the reliability of the REE system at a time $t=5$ years.

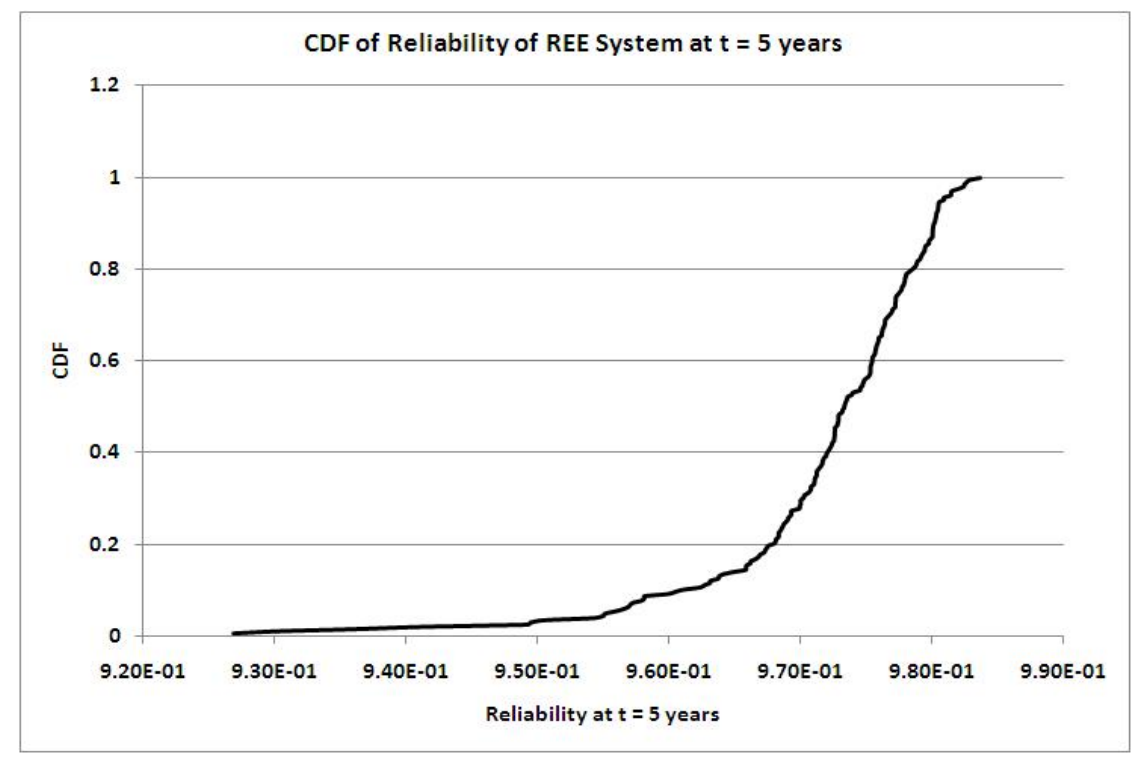

FigurE 6.3: CDF of Reliability of REE System at $t=5$ years 
The $5^{\text {th }}$ percentile from the CDF provides the lower limit for the $95 \%$ upper one-sided confidence interval of reliability, which is computed to be $(0.955861,1)$. The upper and lower limits of the two-sided 95\% confidence interval of reliability are provided by the $97.5^{\text {th }}$ percentile and $2.5^{\text {th }}$ percentile, respectively. The two-sided $95 \%$ confidence interval of reliability is computed to be $(0.949485,0.981994)$.

The robustness of LHS procedure was compared to the random sampling procedure by performing 20 runs of the entire uncertainty propagation method, each run with 208 samples from each epistemic distribution, by both the LHS sampling procedure as well as the random sampling procedure. The variance of sample mean of the reliability at time $t=5$ years, computed in each of the 20 iterations, was computed as an indicator of the robustness of the sampling procedure. This variance obtained by using LHS sampling procedure in the uncertainty propagation method was $4.17 e-8$, which was less than that computed by using the random sampling procedure as $1.56 e-7$. The empirical CDF of means of reliability obtained in the 20 executions by both the sampling procedures are summarized in Figure 6.4. It can be shown that the variance of other statistics and quantiles of the distribution of reliability will also have lower variance with LHS procedure. Thus in this example, LHS provides a more robust sampling procedure.

Next we compare the time taken to perform the epistemic uncertainty propagation through the hierarchical reliability model of the REE system by using LHS method, to that taken when using the random sampling procedure. The time taken was determined using AbsoluteTiming function of Mathematica [2], which provides the real (wall clock) time, ignoring any time taken in printing or formatting of the outputs. At the sample number of samples, the difference in time taken between the two sampling procedures can be attributed to the extra steps in LHS for dividing the probability space and performing random pairings without replacement between samples from different epistemic distributions (the number of executions of the aleatory 


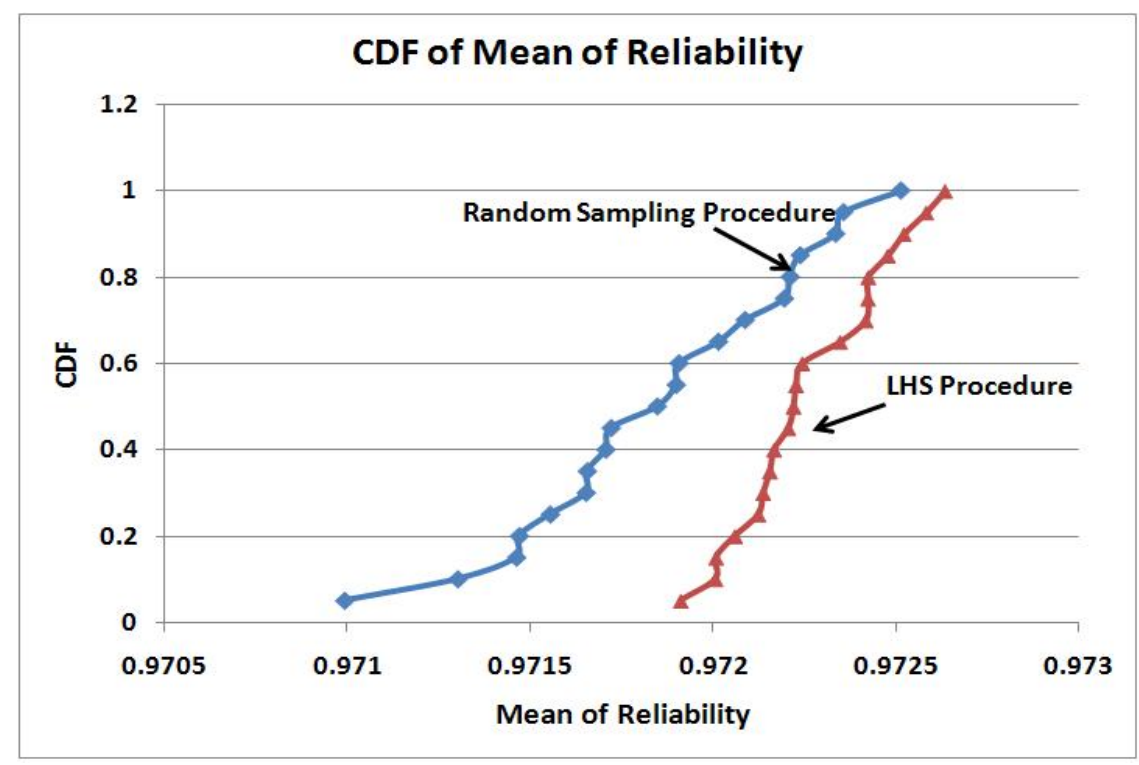

Figure 6.4: CDF of Mean of Reliability: LHS Vs. Random Sampling

model and the number of samples from each epistemic distribution being the same in both cases). However, since LHS requires fewer samples to provide a more robust estimate and cover the entire probability space, the computational savings in drawing fewer samples from the epistemic distributions and fewer executions of the aleatory model can more the compensate for the computational time taken to divide the probability space and perform random pairings without replacement between samples from different epistemic distributions. For epistemic uncertainty propagation through the hierarchical reliability model of the REE system, the time taken by LHS method was 158.78 seconds, while that by the random sampling method was 149 seconds.

\subsubsection{Reliability and mean response time of composite web service}

We perform epistemic uncertainty propagation by LHS method, through the service reliability and performance model of a composite web service called Travel-Agent, which was discussed in [75]. This example was already considered in Section 5.2.4 in context of epistemic uncertainty propagation using random sampling method. 
Therefore, in this section, we skip the common steps of determining the form and parameters of epistemic distribution of each of the model input parameters as random variables and computing the number of samples from each epistemic distribution.

As explained in Section 5.2.4, the number of samples to be drawn from each of the epistemic distributions, is computed as the largest of the number of observations that would have been used to compute the point and interval estimates, across all the model input parameters. Since, the number of observations (or number of attempted executions of services), for parameters used in response time computation of the composite web service, range from 67 to 421, the number of samples to be drawn from the epistemic distribution of each input parameter is determined to be $n=421$. The number of observations computed for the parameters used in overall reliability computation range from 67 to 123 and hence the number of samples to be drawn from the epistemic distribution of each input parameter is determined to be $n=123$, for this example.

We follow the Latin Hypercube Sampling procedure as explained in Section 6.1. To implement the LHS sampling procedure, we use Mathematica's built-in functions RandomReal, Quantile and RandomSample, in the same manner as discussed in the previous examples. However in this case, the CTMC models can be solved to obtain closed-form solutions for both the mean service response time as well as the overall service reliability. Therefore, at each of the $n$ sets of values of the parameters, we evaluate the closed-form expressions using Mathematica (unlike using SHARPE to solve the model as in the first example, where closed-form solution did not exist).

Figure 6.5 shows the empirical CDF of the mean service response time, taking into account the restarts of processes and overhead of time spent in retries, constructed from the $n=421$ values of output obtained by the LHS sampling procedure. Based on this CDF, the $95 \%$ two-sided confidence interval of mean service response time of the composite web service is calculated to be $(7.6730,8.3856)$. 


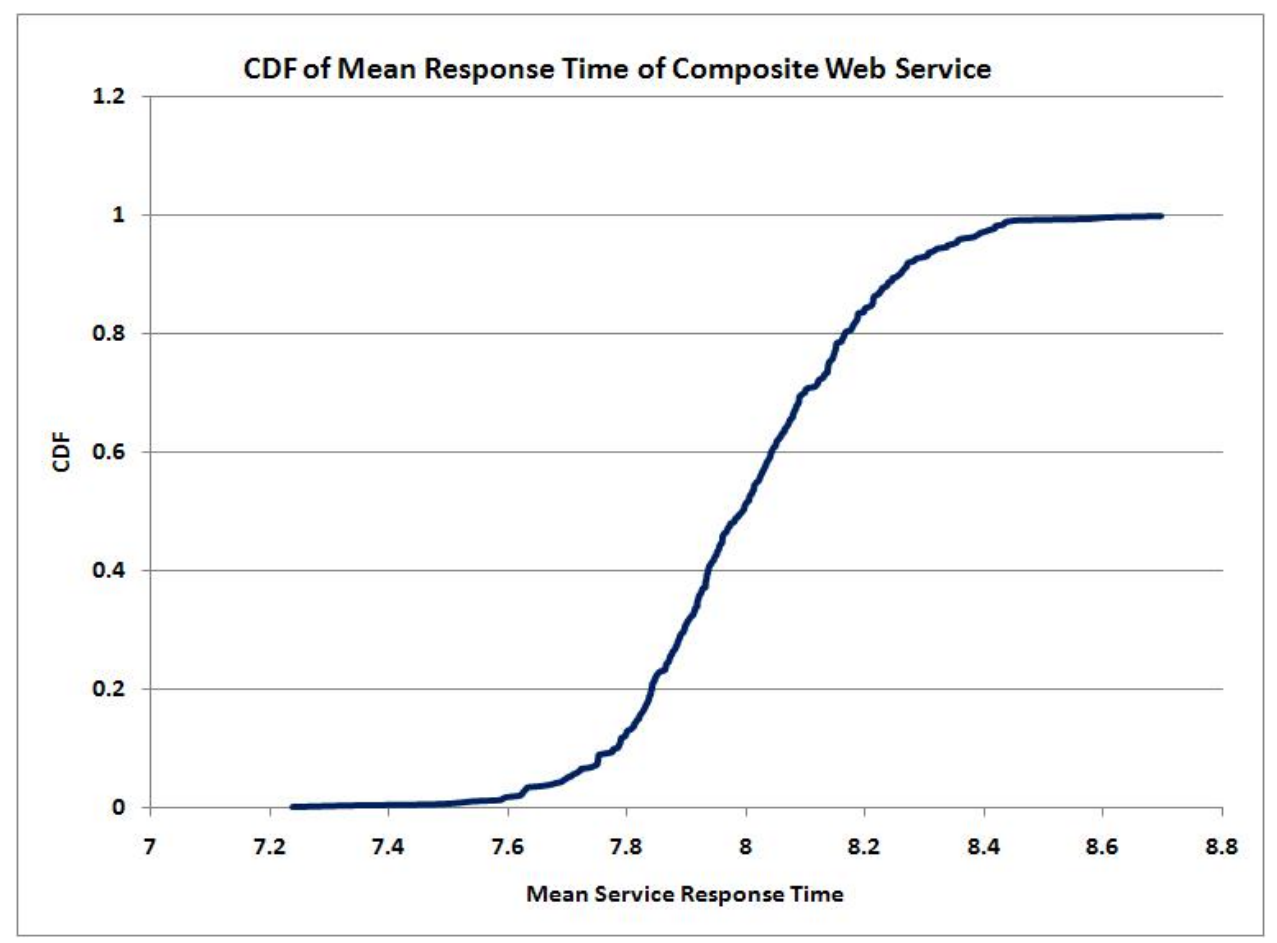

Figure 6.5: CDF of Mean Service Response Time

The closed-form expression for overall reliability of the composite web service, $R_{\text {sys }}$, upon considering perfect coverages for airline reservation (not airline selection) and hotel reservation and perfect reliability for customer reply and service initialization (parameter values as considered for the reliability computation in [75]), reduces to:

$$
R_{\text {sys }}=R_{a 1}+R_{a 2}-R_{a 1} \cdot R_{a 2}
$$

The epistemic density function for $R_{a 1}$ and $R_{a 2}$ are determined to be beta distribution with parameters 99 and 26. Then as explained in Equation 3.1, the expression for 
CDF of the overall reliability of the composite web service, $R_{\text {sys }}$, can be obtained as:

$$
\begin{aligned}
F_{R_{s y s}}(r)= & \int_{0}^{1} \int_{0}^{1} I\left(R_{\text {sys }} \leq r\right) \underbrace{\left(\frac{\Gamma(99+26)}{\Gamma(99) \Gamma(26)} r_{a 1}{ }^{99-1}\left(1-r_{a 1}\right)^{26-1}\right)}_{\text {beta pdf }} \\
& \underbrace{\left(\frac{\Gamma(99+26)}{\Gamma(99) \Gamma(26)} r_{a 2}{ }^{99-1}\left(1-r_{a 2}\right)^{26-1}\right)}_{\text {beta pdf }} d r_{a 1} d r_{a 2}
\end{aligned}
$$

Figure 6.6 shows the empirical CDF of the service reliability, obtained by using the LHS sampling procedure as well as by formal integration of the closed-form expression for service reliability (evaluating the integral at $n=123$ values of reliability), as shown in Equation (6.3). As is clear from the figure, the CDF, variance and percentiles obtained by both formal integration method and the LHS sampling method are very close. Thus the sampling procedure provides an accurate way to compute the CDF and the confidence interval of service reliability. In this case, since the aleatory model is relatively simple, repeated solutions of the model is not computationally prohibitive. As a result, the repeated numerical integrations take longer time than the sampling based uncertainty propagation method. Performing the epistemic uncertainty propagation using the same computing system as explained in Section 6.2.1, the time taken in the LHS sampling based approach was 0.484 seconds while the repeated numerical integrations took 47.76 seconds.

The $95 \%$ two-sided confidence interval of reliability of the composite web service (without considering restarts) is calculated to be $(0.93818,0.9719)$.

We then evaluate the robustness of results obtained by both LHS and random sampling procedure, in our method. We compute the variance of sample mean, calculated over 50 runs of the entire uncertainty propagation method, using both the sampling procedures. Table 6.1 shows that LHS provides a lower variance for the sample mean of the response time as well as service reliability, computed over 50 


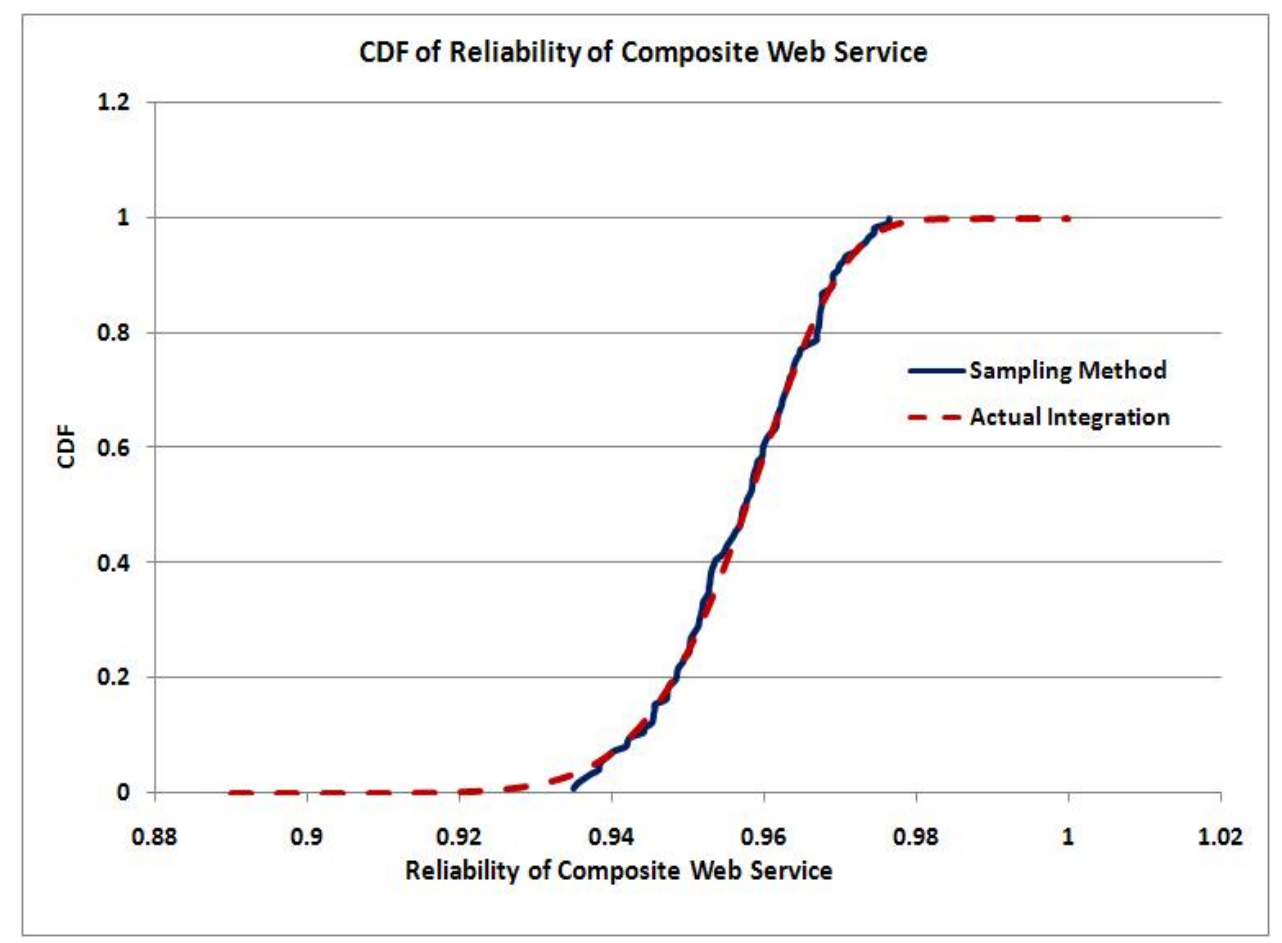

Figure 6.6: CDF of Reliability of Composite Web Service

runs of the entire uncertainty propagation method. Therefore, in this example too, LHS provides a more robust sampling method.

Table 6.1: Variance of Sample Mean

\begin{tabular}{|c|c|c|}
\hline Output Metric & Sampling Procedure & Variance of Sample Mean \\
\hline Mean Resp. Time & Random & $8.35 \mathrm{E}-05$ \\
\hline Mean Resp. Time & LHS & $3.10 \mathrm{E}-07$ \\
\hline Service Reliability & Random & $7.79 \mathrm{E}-07$ \\
\hline Service Reliability & LHS & $1.38 \mathrm{E}-08$ \\
\hline
\end{tabular}

\subsubsection{Capacity Oriented Availability of CMTS System}

We consider uncertainty propagation through the stochastic reward net (SRN) model of the cable modem termination system (CMTS) cluster system consisting of $N$ active nodes and one standby node, described in [43], as the next example to illustrate the applicability of Latin Hypercube Sampling based epistemic uncertainty propagation. 
The CMTS provides an interface between the cable network and the Internet for the traffic of a cable modem based broadband internet access system. It also provide the quality of service (QoS) as well as billing and subscriber authorization services. This system comprises of very complex hardware and software components. To ensure its high availability, hardware redundancy and software resiliency is employed. Figure 6.7 shows a high availability CMTS cluster. It consists of $N$ primary CMTS (PCMTS) nodes and one secondary CMTS (SCMTS) node. In this example, we consider the SCMTS to be a hot standby node for the $N$ primary active nodes. Upon failure of any PCMTS node, the traffic from the failed PCMTS node is switched over to the SCMTS node, which now acts as an active node and repair is initiated $\mathrm{n}$ the failed PCMTS node. Upon completion of repair of PCMTS node, it is switched back in and SCMTS is switched out back. the switch-overs are carried out by hybrid fiber coaxial (HFC) interface switch (HIS).

The SRN Model of CMTS considers both hardware as well as software failures. Software failures caused due to Mandelbugs and aging-related bugs [26] are considered. Hardware failures are assumed to be automatically detected and repaired. The software failures are assumed to be either automatically detected or manually detected (with some probability). Detection delays as well as time to repair are considered for both hardware and software failures. Switchover (and switchover delays) after failure to the standby node and switch back after repair, are considered in case of both hardware and software failures. For details on the CMTS system and the SRN model, the reader is referred to [43]. The model output metric considered for epistemic uncertainty propagation is capacity oriented availability (COA) which is computed as a ratio of average number of available primary CMTS nodes to the the total number of primary CMTS nodes in the cluster. For ease of reference the SRN model of CMTS cluster system, without rejuvenation, has been reproduced below in Figure 6.2.4. 


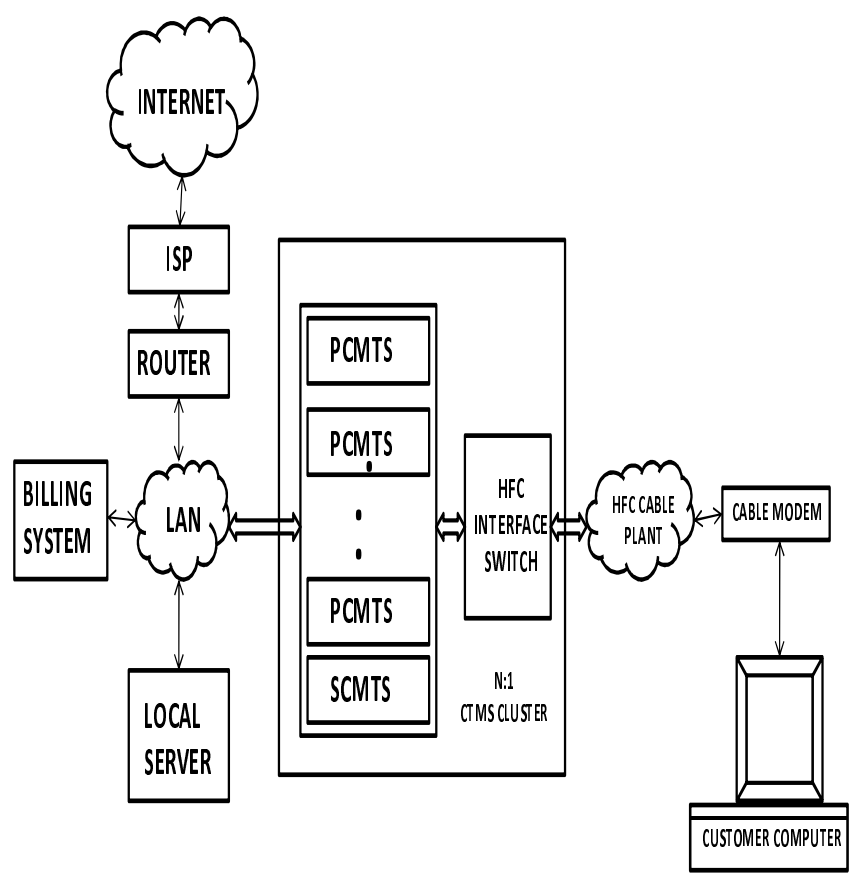

Figure 6.7: CMTS Cluster System Architecture (adapted from [43])

For the uncertainty propagation through the SRN model of CMTS system without rejuvenation, we assume that the uncertainty in parameter values (epistemic uncertainty) is presented in the form of confidence interval of input parameter values (and not the epistemic distribution of the parameters) and that we do not have direct access to the lifetime data or fault injection experiment data for the system. Hence, the form and parameters of epistemic distribution of each of the input parameters as random variables (in this example assuming epistemic independence) needs to be determined first, as explained in Section 5.1.1, based on the point estimate and the input confidence interval of each parameter. 


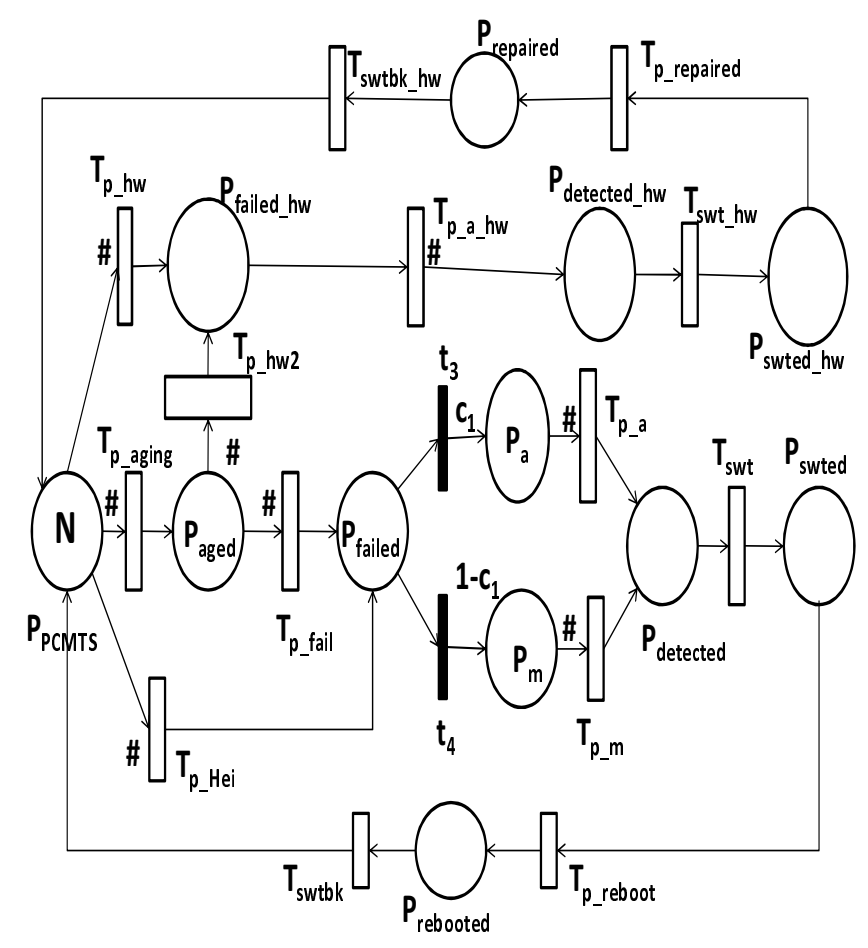

(a) PCMTS

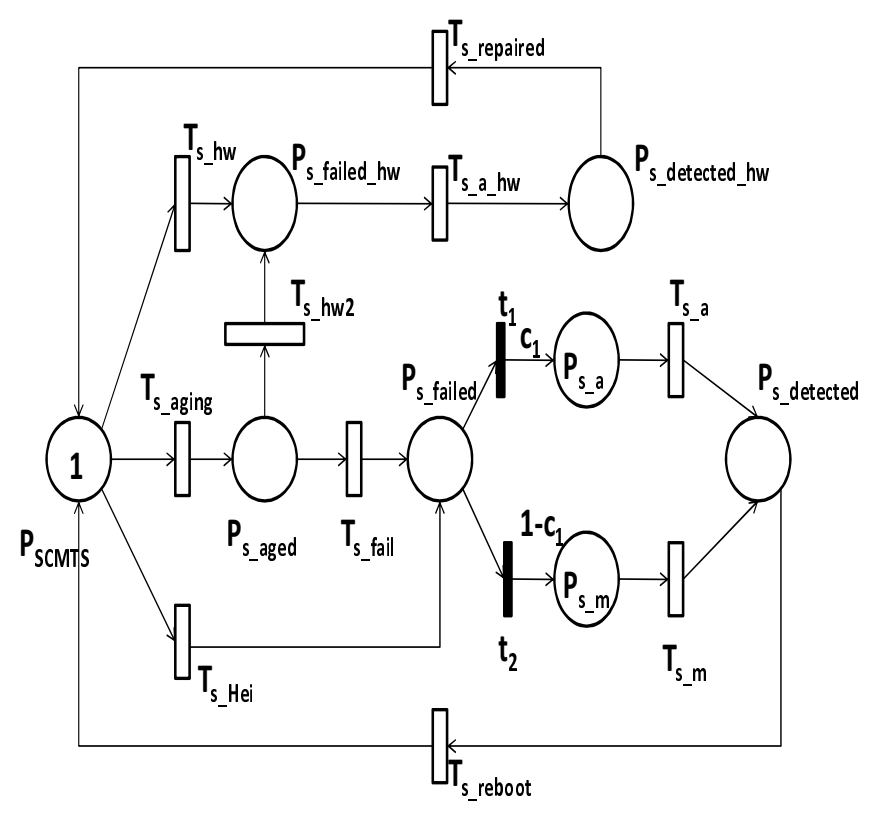

(b) SCMTS

Figure 6.8: SRN model of CMTS Cluster System without Rejuvenation (adapted from [43]) 
The point estimates of parameters of the SRN are taken from [43], while the half width of the $95 \%$ two-sided confidence interval of the rate parameters in the model are assumed to at most $10 \%$ of the point estimate, the lower limit of $95 \%$ upper one-sided confidence interval of the coverage parameter is assumed to be $90 \%$ of the point estimate. In order to determine the form and parameters of epistemic distributions of each of the model input parameters as random variables, the number of observations that would have been used to compute the point estimate and the confidence interval of input parameters, are computed to range from 46 to 165 . Using the number of observations thus computed, the parameters of the Erlang epistemic density function of each rate parameter is derived as shown in Equation 3.4 and the parameters of beta epistemic density function of the coverage parameter is derived as shown in Equation 2.14.

Once the form and parameters of epistemic density functions are determined, latin hypercube sampling (LHS) is employed and samples are drawn from the epistemic distributions of each of the input parameters as random variables. The number of samples needed from the distribution of each input parameter is determined to be $\mathbb{N}=165$, largest of all the number of observations that would have been used to compute the point and interval estimates of the input parameters of the aleatory model. To implement the LHS procedure, we use Mathematica's built-in functions RandomReal, Quantile and RandomSample. At each of the $\mathbb{N}=165$ set of sampled values of the parameters, the SRN model is solved using the software package SPNP [32], to obtain $\mathbb{N}$ values for the model output (capacity oriented availability). Empirical cumulative distribution function (CDF) [86] of the model output measure, is constructed out of the set of $\mathbb{N}$ output values thus obtained. Confidence intervals of the model output, due to the epistemic uncertainties in the model input parameters, are computed by choosing the appropriate percentiles from the CDF (for example, values of the model output corresponding to $2.5^{\text {th }}$ and $97.5^{\text {th }}$ percentile, provide the 
limits of the $95 \%$ two sided confidence interval of the model output). Figure 6.9 shows the empirical CDF of the capacity oriented availability of the CMTS cluster system, constructed from the $\mathbb{N}=165$ values of model output. With the assumed

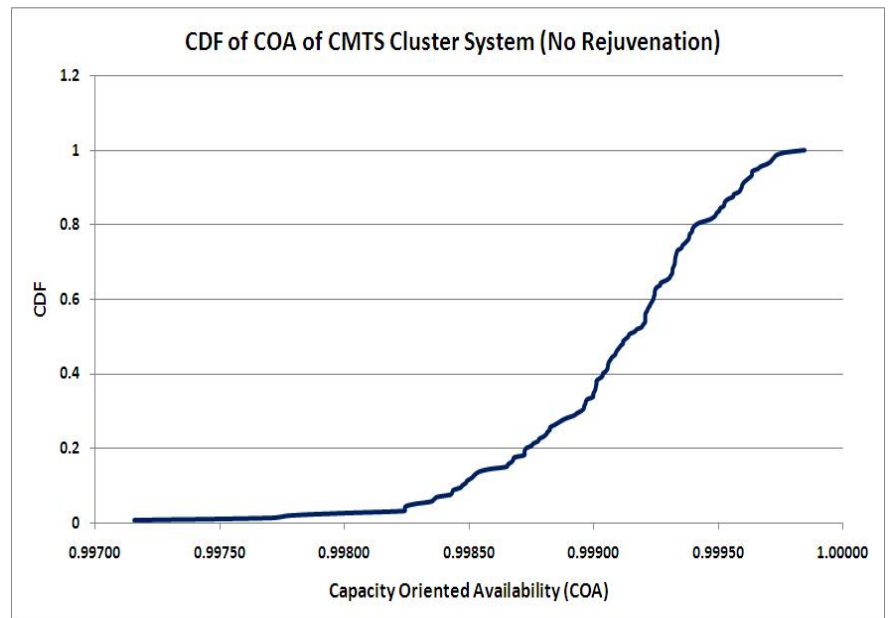

Figure 6.9: CDF of COA of the CMTS Cluster System without Rejuvenation

confidence intervals of the model input parameter values, the expected value of the COA is calculated to be 0.9990776 and the variance is $0.2 E-06$. Using the appropriate percentiles from the empirical CDF in Figure 6.9, the 95\% two-sided confidence interval is computed to be $(0.9979764,0.9997159)$.

\subsubsection{Fixed-Point Iterative Model of a Communication Network}

As yet another example to illustrate the versatility of LHS based epistemic uncertainty propagation, we consider the performance model of a single cell in a cellular wireless communication network, discussed in [27]. The model is solved using a fixed point iteration method. Poisson arrival at a rate $\lambda_{1}$ of new calls and at a rate $\lambda_{2}$ for the handoff calls into the cell is assumed. An ongoing call in the cell (new or handoff) is assumed to complete at rate $\mu_{1}$ (and have an exponential call time) and a call is assumed to move to another cell at rate $\mu_{2}$. Each cell has $N$ channels. A handoff call is accepted if there any idle channels in the cell. However, a new call is accepted 
only if there are more than $g+1$ channels idle, otherwise the new call is blocked. $g$ is said to be the number of guard channels. To not exclude new calls completely $g \leq N$. The CTMC model of this wireless communication cell was presented in [27] and is reproduced below for ease of reference. The arrival and departure rates in the above CTMC, are state-dependent. The state of system is denoted by $n$, the number of busy channels. The state dependent rates are given by:

$$
\Lambda(n)=\left\{\begin{array}{lr}
\lambda & n=0,1, \ldots, N-g-1 \\
\lambda_{2} & n=N-g, \ldots, N-1 ; g>0
\end{array}\right.
$$

and $M(n)=n \mu, n=1, \ldots, N$, where $\mu=\mu_{1}+\mu_{2}$.

New calls originating in the cell when more than $N-g-1$ channels are busy, are blocked. Therefore, the blocking probability of new calls, $P_{b}$ is the sum of probabilities of being in states $(N-g)$ through $N$. Calls handed-off from other cells are dropped only if all the $N$ channels are busy. Therefore, the dropping probability of hand-off calls, $P_{d}$ is the probability of being in state $(N)$.

Since the number of channels $N$ is usually large and the expression for $P_{d}$ and $P_{b}$ involves factorials and exponents, computing their values presents computational difficulties. Recursive formulas shown in Equations 6.5 through 6.7 have been presented in $[27]$ to compute these probabilities.

$$
E_{B}(A, k)=\frac{\frac{A}{k} E_{B}(A, k-1)}{1+\frac{A}{k} E_{B}(A, k-1)}, \quad k=1,2, \ldots, N
$$

with $E_{B}(A, 0)=1.0 . E_{B}(A, k)$ is the loss probability with traffic intensity $A=\lambda / \mu$ and total $k$ channels (This is a recursive version of the Erlang-B loss formula).

The dropping probability of handoff calls, $P_{d}(N, g)$ in a cell with total $N$ channels and $g$ guard channels is computed recursively as:

$$
P_{d}\left(N_{1}+k, k\right)=\frac{P_{d}\left(N_{1}+(k-1), k-1\right)}{\frac{N}{\alpha A}+P_{d}\left(N_{1}+(k-1), k-1\right)}, \quad k=1,2, \ldots, g .
$$


with $P_{d}\left(N_{1}, 0\right)=E_{B}\left(A, N_{1}\right)$. Where $N_{1}=N-g$ and $\alpha=\left(\lambda_{2} \mu\right) /\left(\lambda\left(\mu_{1}+\mu_{2}\right)\right)$.

The new call blocking probability $P_{b}(N, g)$ in a cell with total $N$ channels and $g$ guard channels is computed recursively as:

$P_{b}\left(N_{1}+k, k\right)=\frac{\frac{N}{\alpha A} P_{b}\left(N_{1}+(k-1), k-1\right)+P_{d}\left(N_{1}+(k-1), k-1\right)}{\frac{N}{\alpha A}+P_{d}\left(N_{1}+(k-1), k-1\right)}, k=1,2, \ldots, g$.

with $P_{b}\left(N_{1}, 0\right)=E_{B}\left(A, N_{1}\right)$.

In Equations 6.5 through 6.7, the handoff call arrival rate $\lambda_{2}$ has been assumed to be given. But in real-life the value of $\lambda_{2}$ needs to be estimated. In [27], existence of fixed point has been demonstrated and a fixed point equation for $\lambda_{2}$ is derived as:

$$
x=\frac{\mu_{2} \lambda_{1}\left(1-P_{b}(x)\right)}{\mu-\mu_{2}\left(1-P_{d}(x)\right)}
$$

where, $P_{b}(x)$ and $P_{d}(x)$ are call blocking and dropping probabilities, respectively, as a function of handoff call arrival rate $x$. The initial assumption is $x=\mu_{2} \lambda_{1} / \mu_{1}$.

\section{Uncertainty Propagation through Fixed Point Iteration Model}

The point estimates of the parameters in the Equations 6.5 through 6.8 were obtained from [27]. The total number of channels $N$ is assumed to be 100 and number of guard channels $g$ is assumed to be 3 . The point estimate of new call arrival rate $\lambda_{1}$ is taken to be 40 per unit time and the completion rates of both handoff and new calls is assumed to be $\mu_{1}=\mu_{2}=0.5$, per unit time. The epistemic uncertainty is assumed to be presented in the form of confidence intervals of each of the input parameters. The half-width of $95 \%$ two-sided confidence interval of the parameters is assumed to be at most $10 \%$ of the point estimate.

Since all the parameters in the fixed point iterative model are rate parameters of exponential distribution, the epistemic density function of each of the aleatory model input parameter is Erlang, as shown in 3.4. The number of samples to be drawn from 
each epistemic distribution is computed to be $n=269$, the largest of all the number of observations that would have been used to compute the point estimates of the aleatory model input parameters.

The uncertainty propagation wrapper as well as the recursive and the floating point iterative equation are implemented in Mathematica. For each of the $n=269$ set of input parameters values obtained by LHS sampling, first Equations 6.5 through 6.7 are solved recursively and then Equation 6.8 is solved for each value of $\lambda_{2}$ (starting at the initial assumption of value for $\lambda_{2}$,), until convergence in value of $\lambda_{2}$, between two successive solutions of Equation 6.8, is reached.

An empirical CDF is constructed out of the $n=269$ values of $\lambda_{2}$ thus obtained. Figure 6.10 shows the empirical CDF of the handoff call arrival rate. The $95 \%$ twosided confidence interval of the handoff call arrival rate $\lambda_{2}$, due to the epistemic uncertainty in the input parameters of the fixed point iteration model is obtained as $(32.56,45.77)$.

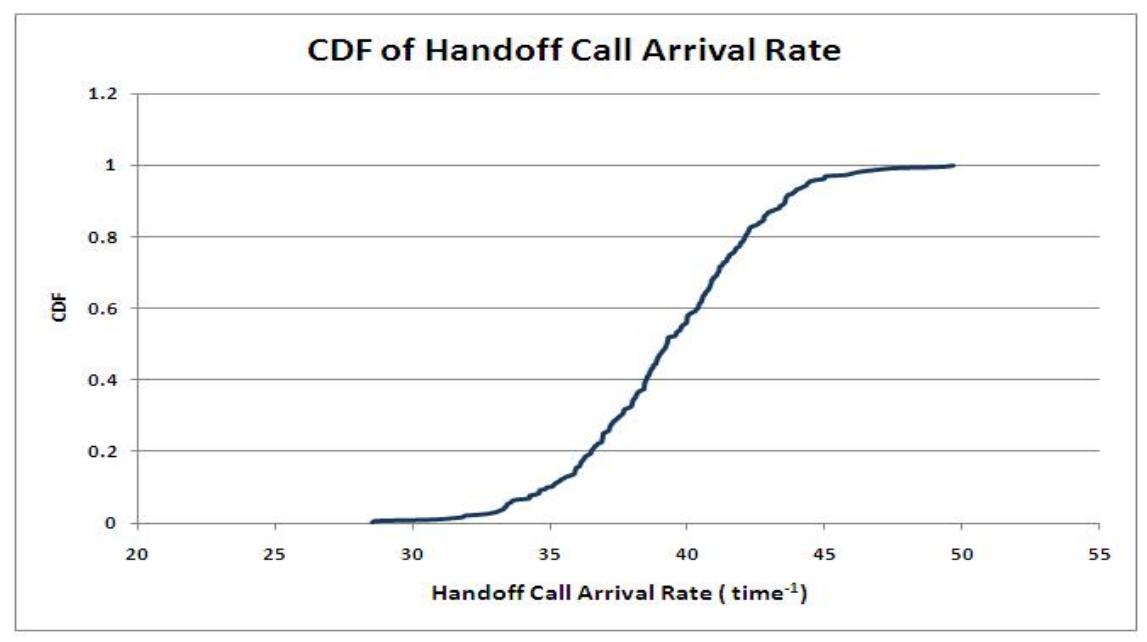

FiguRE 6.10: CDF of handoff call arrival rate 


\section{7}

\section{Correlated Parameters}

In the uncertainty propagation method presented in Chapters 3 through 6, the model input parameters are considered to be independent random variables. This assumption of epistemic independence simplifies the epistemic uncertainty propagation as it allows for using the product of marginal densities in place of the joint epistemic density of all the aleatory model input parameters as random variables. This simplifies sampling of parameter values in the sampling based method as well as the unconditioning integrations (Equation 3.1 through 3.3) in the closed-form and analyticnumerical uncertainty propagation methods described in Chapters 3 and 4, respectively.

However, in real life, there may be dependencies in the aleatory model input parameter values as random variables, originating from the fact that the parameter values may be obtained from a common data or information source. Correlations provide a way to measure dependence between random variables. Ignoring correlations between parameters can lead to errors and biases in the output metrics, depending on the degree of correlation $[81,12]$. It has been argued that the dependency between model parameter values arising out of commonality in data or information 
sources can be more intuitively captured by rank correlations rather than direct correlation between parameter values $[28,35,29]$. Therefore, in this chapter, we relax the assumption of epistemic independence by considering rank correlation between the aleatory model input parameters as random variables.

It should be noted that if the correlation is not very strong or if the model output is not very sensitive to the correlated parameters, the distribution of model output may not be affected in a significant way $^{1}[81,12]$.

\subsection{Rank Correlation Among Input Parameters}

We employ a widely used and distribution free method of imposing rank correlation between parameter values sampled from the marginal epistemic distributions of dependent parameters as random variables, proposed by Iman and Conover [35, 29]. Being distribution free, this method can be applied to any epistemic distribution. It does not require determining and sampling from joint distributions but imposes rank correlations on values already sampled from marginal distributions. It also preserves any stratification introduced by the sampling method (thus retains properties of LHS sampling).

\section{Introducing Correlation between Parameters}

Using the Iman-Conover (IC) method to introduce correlations between values of model input parameters as random variables, sampled from their marginal epistemic distributions, can easily be incorporated into the sampling based method epistemic uncertainty propagation method, explained in Chapters 5 and 6 . The steps for uncertainty propagation explained in Sections 5.1.1 through 5.1.5, with an exception to generation of the sets of model input parameters discussed in Section 5.1.3, stay the same. Once the marginal epistemic distributions (type and parameters) of each of

\footnotetext{
${ }^{1}$ Adapted from [54]
} 
the $k$ aleatory model input parameter as random variable is determined, the number of samples $n$, to be drawn from each of these epistemic distribution is computed. Then latin hypercube sampling (LHS) is performed as explained in Section 6.1 to generate an $n-b y-k$ matrix of sampled parameter values, $\boldsymbol{V}$ shown in Equation 6.1. The next step is to introduce desired correlation among the parameter values thus sampled. The desired correlation is provided in the form a $k-b y-k$ matrix $\boldsymbol{S}$, where each element $s_{i j}$ is the value of rank correlation between the $i^{\text {th }}$ and $j^{\text {th }}$ input parameter as random variable, $\Theta_{i}$ and $\Theta_{j}$, respectively $(i, j \in 1,2, \ldots, k)$.

The IC method takes the $n-b y-k$ matrix of sampled parameter values, $\boldsymbol{V}$ (matrix shown in Equation 6.1) and the desired rank correlation matrix $\boldsymbol{S}$ (a $k-b y-k$ matrix) as its input. It is based on the result that the product of an orthogonal matrix, in which each column has mean of zero and standard deviation of one (matrix with Van der Waerden scores [35] used here), with the upper triangular matrix of desired correlation matrix (obtained by Cholesky decomposition), results in a matrix with ordering of elements in a column to be same as what would give the desired rank correlation matrix. Simply rearranging the sampled values (obtained by LHS) from each epistemic distribution, in the same order as this new matrix, ensures that the input parameters have the desired rank correlation. The steps in introduction of correlation are :

1. Perform Cholesky decomposition of the desired correlation matrix $\boldsymbol{S}$ to obtain an upper triangular matrix $\boldsymbol{C}$, such that. $\boldsymbol{S}=\boldsymbol{C}^{\boldsymbol{T}} \boldsymbol{C}$, where, $\boldsymbol{C}^{\boldsymbol{T}}$ is the transpose of $\boldsymbol{C}$.

2. Generate an $n-b y-k$ matrix, $\boldsymbol{M}$, such that its elements are values of independent variables and each column has mean zero and standard deviation of one. This is accomplished by using random rearrangements of Van Waerden scores (quantiles of standard normal distribution), as elements of each column 
of this matrix.

3. Generate a matrix $\boldsymbol{N}$, where $\boldsymbol{N}=\boldsymbol{M C}$. It can be shown that this matrix will have the same rank correlation between its columns, as specified between parameters, in the desired rank correlation matrix $\boldsymbol{S}$ [51].

4. Simply re-arrange the elements of each column in the matrix $\boldsymbol{V}$ in the same rank order as the elements in the corresponding column of matrix $\boldsymbol{N}$. This re-arrangement imposes the desired rank correlation between parameters, as specified in $\boldsymbol{S}$.

Rank correlation between any number of input parameters can be considered by this method, as long as the correlation matrix $\boldsymbol{S}$ reflects the various correlations. Ignoring correlations between parameters can lead to errors and biases in the output metrics, depending on the degree of correlation and the parameters which are correlated. If the correlation is not very strong or if the model output is not very sensitive to the correlated parameters, the distribution of model output may not be affected in a significant way $[81,12]$.

For an $n-b y-k$ matrix $\boldsymbol{A}$ where the $j^{\text {th }}$ column contains $n$ sample elements from a random variable $X_{j}(j=1,2, \ldots, k)$, the covariance matrix of the random variables $X_{j}(j=1,2, \ldots, k)$ is given by $\boldsymbol{B}^{\boldsymbol{T}} \boldsymbol{B}$. The matrix $\boldsymbol{B}$ is the matrix obtained by subtracting the mean of $n$ elements in column $j$ from each element in the $j^{\text {th }}$ column of matrix $\boldsymbol{A}$.

In the matrix $\boldsymbol{M}$ above, since each column has a zero mean, the covariance matrix is given by $\boldsymbol{M}^{\boldsymbol{T}} \boldsymbol{M}$. As the matrix $\boldsymbol{M}$ has uncorrelated columns, the covariance matrix of $\boldsymbol{M}$ is given by $\boldsymbol{M}^{\boldsymbol{T}} \boldsymbol{M}=\boldsymbol{I}$, where $\boldsymbol{I}$ is a $k-b y-k$ identity matrix. This covariance matrix is also the correlation matrix because standard deviation of each columns of $\boldsymbol{M}$ is one. Based on this reasoning, the following equations illustrate the workings of Iman-Conover method of imposing rank correlation between 
parameter values sampled from marginal epistemic distributions of aleatory model input parameters as random variables.

$$
\begin{aligned}
N & =M C \\
\operatorname{Correlation}(\boldsymbol{N}) & =N^{T} N \\
& =(M C)^{T}(M C) \\
& =C^{T} M^{T} M C \\
& =C^{T} I C \\
& =C^{T} C=S
\end{aligned}
$$

Since the columns of matrix $\boldsymbol{N}$ are linear combinations of the columns of $\boldsymbol{M}$ (which have zero mean by assumption), the columns of $\boldsymbol{N}$ too have zero mean.

The $j^{\text {th }}$ column in matrix $\boldsymbol{M}$ has elements $m_{i j}(i=1,2, \ldots, n)$ such that $\sum_{i=1}^{n} m_{i j}=$ 0 and $\sum_{i=1}^{n} m_{i j}{ }^{2}=1$. In the IC method, the $i^{\text {th }}$ element of the first column is generated by taking the $i /(n+1)^{\text {th }}$ quantile from standard normal distribution (known as Van der Waerden scores). The rest of the columns are obtained by performing random permutations of the first column [51]. In case the correlation matrix for $\boldsymbol{M}$ does not result in an identity matrix, a correction is applied by setting $\boldsymbol{N}=\boldsymbol{M} \boldsymbol{E} \boldsymbol{C}$. If $\boldsymbol{M}^{\boldsymbol{T}} \boldsymbol{M}=\boldsymbol{T}$ (and not $\boldsymbol{I}$ ), $\boldsymbol{T}=\boldsymbol{D}^{\boldsymbol{T}} \boldsymbol{D}$ and $\boldsymbol{E}=\boldsymbol{D}^{-1}$.

In the rest of this chapter, we illustrate this method of introducing rank correlation between sampled values of input parameters of the aleatory model, obtained by LHS method, with the help of dependability or performance models of real systems.

\subsection{Illustrative Examples}

In this section, we illustrate the applicability of epistemic uncertainty propagation through dependability models, when the aleatory model input parameters as random 
variables are dependent, using Iman-Conover method of imposing rank correlation between input parameter values sampled from their marginal epistemic distributions.

The examples considered are availability model of IBM SIP SLEE server cluster system and reliability model of a composite web service. While the availability model of IBM SIP SLEE server cluster system is a large hierarchical model with 63 input parameters, the reliability model of composite web service is a much simpler CTMC model. We do not manipulate the aleatory model or its output and use the uncertainty propagation wrapper around the pre-existing model solution method. For the IBM SIP server cluster system example, the uncertainty propagation wrapper is implemented in Mathematica while the hierarchical model is solved in SHARPE. For the composite web service example, both the wrapper as well as the model are implemented in Mathematica [87, 2].

\subsubsection{Availability Model of IBM SIP Server Cluster System}

As an example to illustrate the method for epistemic uncertainty propagation by LHS sampling based method, in presence of dependence between model input parameters as random variables, we use the availability of IBM SIP SLEE server cluster, discussed in [85].

Uncertainty propagation through the hierarchical aleatory model of IBM SIP SLEE server cluster, by the random sampling method and LHS, have already been presented in Sections 5.2.2 and 6.2.1, assuming the model input parameters as random variable to be independent (uncorrelated). In this section we relax the assumption of epistemic independence by considering two of the model input parameters to be highly correlated.

The point estimates of the model parameters were obtained from Tables 3 and 4 in [85]. As a measure of the epistemic uncertainty in the model parameter values, we use $95 \%$ two-sided confidence intervals for the rate parameters of the model and $95 \%$ 
upper one-sided confidence interval for the coverage parameters. We choose such values for the limits of the $95 \%$ two-sided confidence interval for the input parameter values, so as to get ratio between point estimate and width of confidence interval to be the same as the ratio between the point estimate and the width of the bounds of parameter values as in [82]. The half width of $95 \%$ two-sided confidence interval for parameters for which the bounds have not been specified in [82] are assumed to be at most $10 \%$ of the point estimate. For the coverage parameters, the lower limit of upper one-sided confidence interval is chosen to be at least $90 \%$ of the point estimate. Since the steps of determining the epistemic uncertainty and the number of samples $n$ from each epistemic distribution are the same as in Section 5.2.2, we skip the details of these two steps here.

The number of samples needed from the epistemic distribution of each of the 63 input parameters is determined to be $n=269$, largest of all the number of observations of the input parameters. We follow the Latin Hypercube Sampling procedure as explained in Section 6.1.

\section{Implementing the IC Method}

While the method explained in Section 7.1 allows considering correlation among any number of input parameters, we consider the failure rates of application server processes and the operating system on the node to be highly correlated (as these were the parameters where epistemic dependence seemed likely, based on the thinking that often times, it maybe difficult to accurately differentiate between failures due to application server and failures due to the operating system), with a rank correlation of 0.7 .

We first perform the LHS procedure to obtain samples from each of the 63 marginal epistemic distributions (Erlang distribution for the failure and repair rate parameters and beta distribution for the coverage parameters of the hierarchical 
aleatory model). To implement the LHS procedure, we first divide the probability space into $n=269$ intervals and then use Mathematica's built-in functions RandomReal and Quantile to generate the random deviates from different intervals in the distribution. We then use the function RandomSample to obtain random pairing without replacement, between samples from different epistemic distributions.

The $n-b y-k(n=269$ and $k=63)$ matrix of sampled parameter values (the matrix $\boldsymbol{V}$ ) and the desired rank correlation matrix $\boldsymbol{S}$, serves as the starting point for the Iman-Conover method. To implement the Iman-Conover method [35] as explained in Section 7.1, in our uncertainty propagation wrapper, we make use of Mathematica's matrix manipulations functions and the function CholeskyDecomposition to get the upper triangular matrix of the correlation matrix. The rearrangement of the sampled parameter values matrix $\boldsymbol{V}$ to have the same rank order as the reference matrix $\boldsymbol{N}$, is carried out as follows:

The Mathematica function Ordering is used on each column $j(j=1,2, \ldots, k)$ of the reference matrix $\boldsymbol{N}$ to obtain the position of successive elements in the column, from smallest to largest. The corresponding column $j$, in the matrix $\boldsymbol{V}$ is first sorted using the function Sort and then its elements are rearranged in positions obtained as a result of using Ordering on the $j^{\text {th }}$ column of $\boldsymbol{N}$. The new matrix $\boldsymbol{W}$ obtained as a result of re-arranging each of the columns in $\boldsymbol{V}$ has the same correlation as provided in the desired rank correlation matrix $\boldsymbol{S}$.

At each of the $n=269$ sets of values of the parameters, the hierarchical aleatory model is solved using SHARPE [87]. Figure 7.1 shows the difference in the shapes of empirical CDF of availability of the IBM SIP server system for the two cases : independent parameter values and correlated parameters. While the $95 \%$ twosided confidence interval of availability in case of all independent parameters was $(0.99999509,0.99999649)$, the confidence interval when two of the parameters are correlated is obtained from Figure 7.1 to be $(0.99994055,0.99997671)$. 
Next we compare the time taken to perform the uncertainty propagation through the hierarchical model, assuming epistemic dependence (correlated parameters) to the case of epistemic independence (no correlation between model input parameter values). The time taken was determined using AbsoluteTiming function of Mathematica, which provides the real (wall clock) time taken, ignoring any printing or formatting of the outputs. The time taken in the case of uncertainty propagation assuming independent parameters (no correlation) was found to be 118.14 seconds, while it was found to be 122.72 seconds for the case of 2 -correlated parameters. The extra time taken in case of correlated parameters can be attributed to the additional steps in inducing the desired correlation between two of the aleatory model input parameters as random variables (perform Cholesky's decomposition on the rank correlation matrix $\boldsymbol{S}$ to obtain the upper triangular matrix $\boldsymbol{C}$; the product $\boldsymbol{N}=\boldsymbol{M C}$; rearrangement of elements in rows of $\boldsymbol{V}$ ). Since only two parameters are considered to be correlated in our case, the rank correlation matrix $\boldsymbol{S}$ is relatively sparse and hence performing Cholesky's decomposition as well as obtaining the product matrix $\boldsymbol{N}$ are not that time consuming. These steps may get prohibitive if rank correlations between a large number of parameters need to be considered.

\subsubsection{Reliability of a Composite Web Service}

As a second example to illustrate the applicability of epistemic uncertainty propagation in presence of epistemic dependence between the aleatory model input parameters as random variables, by the Iman-Conover method, we perform uncertainty propagation through the service reliability of a composite web service called TravelAgent, which was discussed in [75].

The web service Travel-Agent, interacts with different services to make airlines and hotel reservations for customers. Each of the individual interactions (web services) may have complex interactions within themselves and have their own mean 


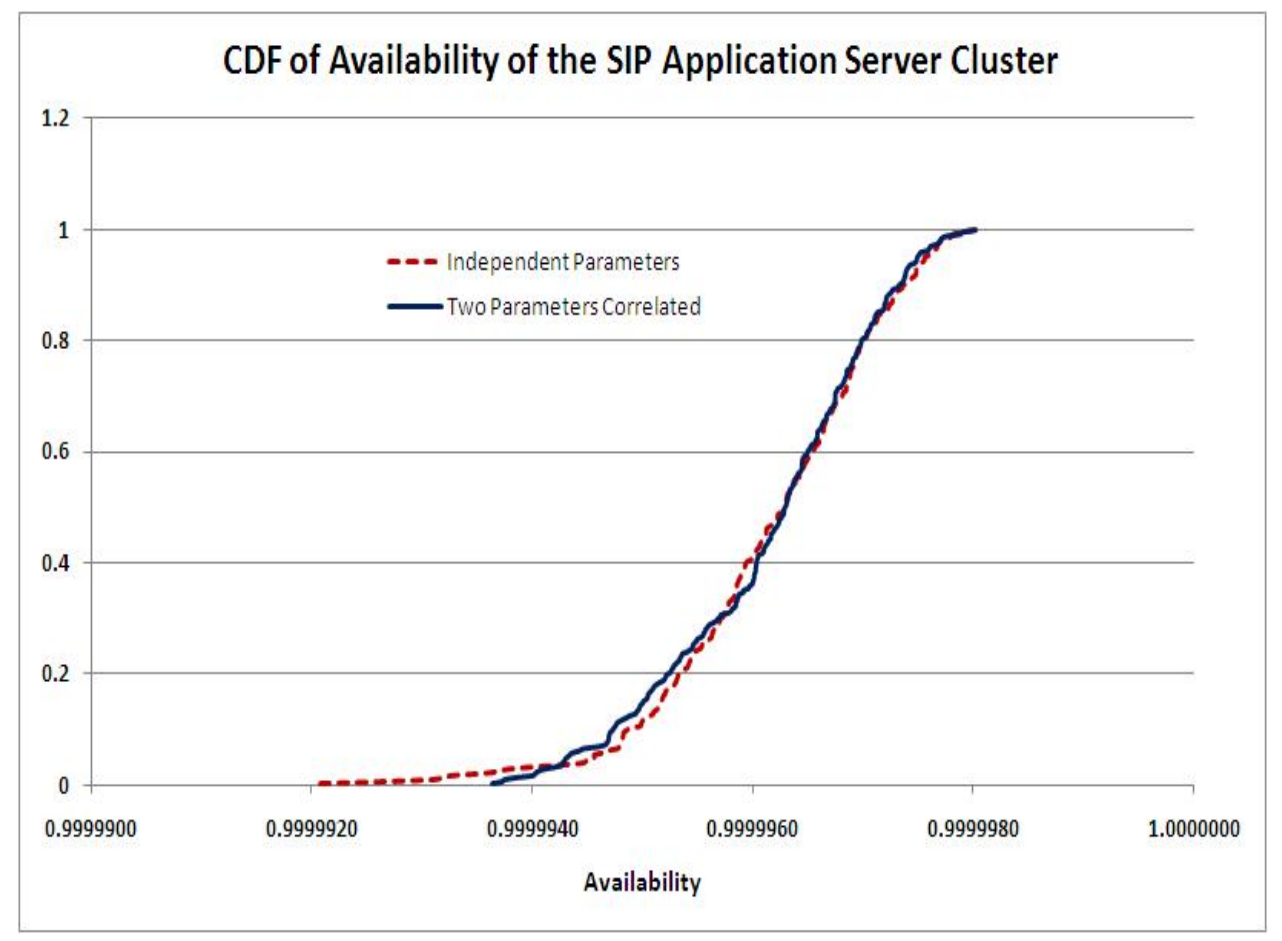

Figure 7.1: CDF of SIP server cluster availability

response time and reliability. When invoked, it first invokes services from two different airlines, in parallel. Upon getting the response from both, it chooses one of them, based on some requirements. If only one of them responds, it chooses the only response. Then it makes airlines reservation and hotel reservation and notifies the user. Each of these operations may fail and can be retried/restarted. The entire process is considered successful only if a successful airline and hotel reservation is made and a reply is sent back to the user. The look up for vacancy in airlines happens concurrently on both airlines.

A CTMC model is formulated to study the overall reliability and response time of the entire composite web service in [75]. The reliability of each of the individual services is computed as the ratio of number of successful executions of the service to the total number of executions of the service attempted and is assumed to follow a Bernoulli distribution. The CTMC considers concurrent execution of the two 
airlines services, failure of any of the services and restarts/retries of services to attempt recovery from failure of a service. It also considers overhead of time spent in restarts (assuming this overhead time to follow an exponential distribution) as well as imperfect coverage of restarts of services.

Propagation of epistemic uncertainty propagation through service reliability and performance model of the composite web service, by random sampling and LHS method have been discussed in Sections 5.2.4 and 6.2.3, respectively (and by closedform analytical as well as analytic-numerical method in Sections 3.8 and 4.1, respectively). While all the analysis so far considered the model input parameters as random variables to be independent of each other, in many cases, there may be dependency between parameter values, arising out of common data or information sources. In this section we consider uncertainty propagation through the reliability model of the composite web service when two of its parameters are correlated. Since most of the steps (except the sampling step) are the same as shown in sampling based uncertainty propagation in Sections 5.2.4 and 6.2.3, we will only briefly go over them here.

The closed-form expression for overall reliability of the composite web service, $R_{\text {sys }}$, upon considering perfect coverages for airline reservation (not airline selection) and hotel reservation and perfect reliability for customer reply and service initialization (parameter values as considered for the reliability computation in [75]), reduces to:

$$
R_{\text {sys }}=R_{a 1}+R_{a 2}-R_{a 1} \cdot R_{a 2}
$$

The epistemic density function for $R_{a 1}$ and $R_{a 2}$ are determined to be beta distribution with parameters 99 and 26.

As explained in Section 5.2.4, the number of samples to be drawn from each of the epistemic distributions, is computed as the largest of the number of observations 
that would have been used to compute the point and interval estimates. The number of observations computed for the parameters used in overall reliability computation range from 67 to 123 and hence the number of samples to be drawn from the epistemic distribution of each input parameter is determined to be $n=123$, for this example.

While all the analysis so far considered parameters to be independent of each other, in many cases, there may be dependency between parameter values, arising out of common data or information sources, which need to be considered. We employ the Iman-Conover method for imposing rank correlation between parameter values already sampled from the marginal distributions of the parameters by LHS method. While rest of the steps in uncertainty propagation stay the same, considering correlation between parameters adds an extra step in the sample generation phase, where Iman-Conover method is applied to the samples already obtained from the marginal epistemic distributions, by the LHS method, as explained in Section 7.1. For the purpose of illustration, we consider a rank correlation of 0.7 between $R_{a 1}$ and $R_{a 2}$, the reliabilities of the two airlines selection processes. The empirical CDF of overall reliability of the composite web service considering independent parameters, as well as correlated parameters, has been shown in Figure 7.2. The $95 \%$ two-sided confidence interval of reliability of composed web service, considering correlated parameters is computed to be $(0.92217,0.97759)$, while the confidence interval for reliability, computed assuming independent parameters, is $(0.92739,0.97678)$.

Using AbsoluteTiming function of Mathematica, the time taken to perform epistemic uncertainty propagation when considering independent parameters (no correlation) was found to be 0.484 seconds, while it was found to be 1.81 seconds for the case of 2-correlated parameters. 


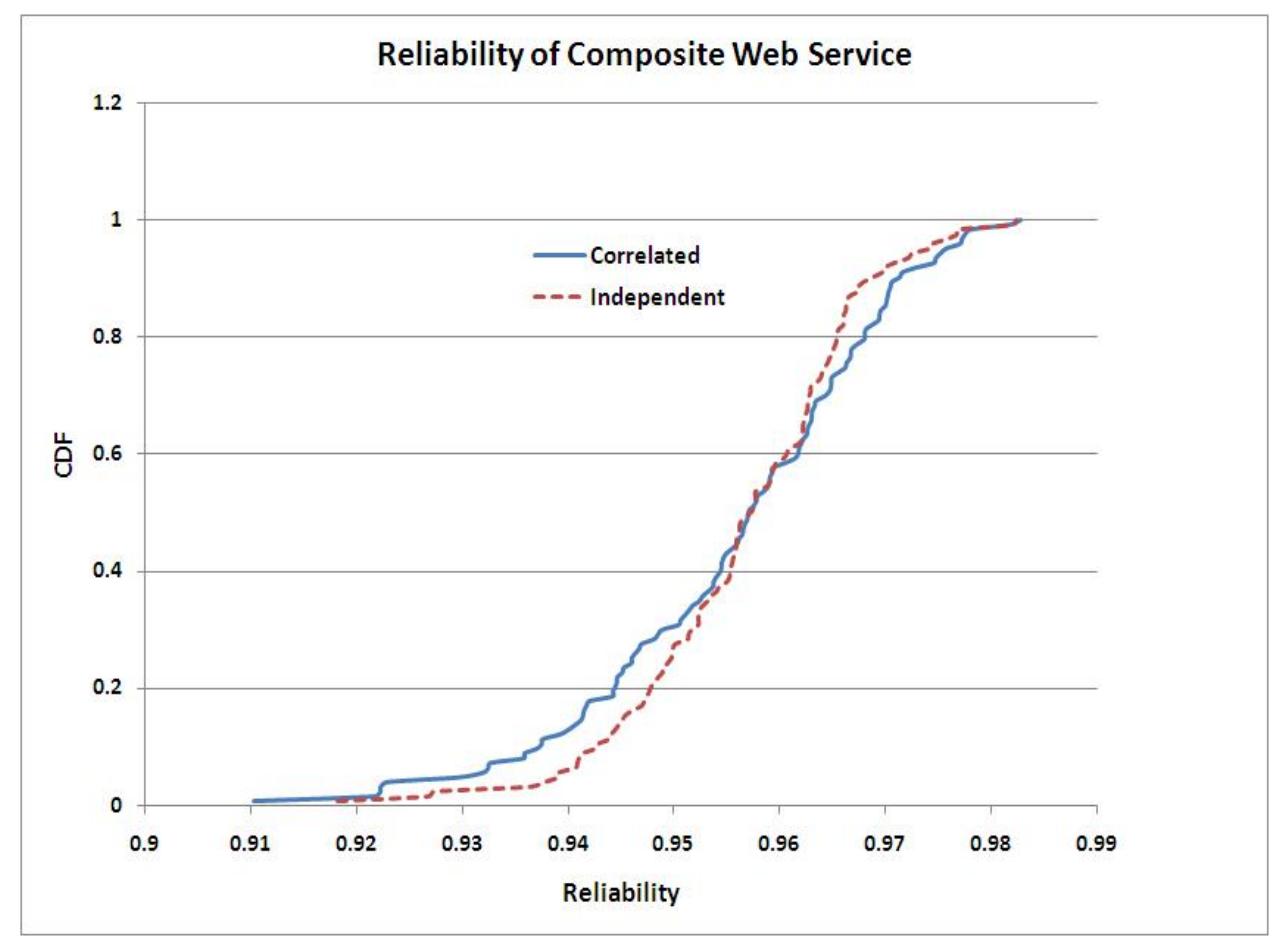

FiguRE 7.2: CDF of Reliability considering independent and correlated parameters 


\section{8}

\section{Aleatory Model with Simulative Solution}

Epistemic uncertainty propagation discussed in Chapters 3 through 7 considers epistemic uncertainty propagation through analytic aleatory models with either analytic or analytic-numerical solutions. In this chapter, we discuss epistemic uncertainty propagation when the aleatory model has a simulative solution.

While the uncertainty propagation methods discussed so far in this dissertation were non-intrusive, uncertainty propagation through aleatory models with simulative solution can be performed both in intrusive and non-intrusive way. Unlike aleatory models with analytic solution, where non-intrusive methods are difficult to implement and can be applied only to a limited range of aleatory models, intrusive uncertainty propagation through aleatory models with simulative solutions, can be performed with an effort comparable to that needed for non-intrusive uncertainty propagation.

Sampling based epistemic uncertainty propagation through analytic aleatory models as discussed in Chapters 5 through 7 can be applied for propagation of epistemic uncertainty through simulation models in a similar fashion. The sampling based uncertainty propagation method will act as a wrapper to an existing model and its solution technique, except that a simulation will be invoked instead of analytic model 
solution. One significant consideration when applying sampling based uncertainty propagation to simulation aleatory models is that while each solution of the analytic aleatory model at a particular set of parameter values sampled from their epistemic distributions, yields one value of the output, each execution of the simulation model will yield a distribution (or confidence interval) of the output metric, due to the finite number of samples from aleatory distributions. Different invocations of the simulation model will give different distributions. Thus there will be two levels of distributions or confidence intervals to be understood, to quantify the uncertainty in the output in case of simulation aleatory models.

Intrusive epistemic uncertainty propagation through a simulative aleatory model can be performed as: during the execution of the simulation model, for each sample to be drawn from the aleatory distribution, the parameter of the aleatory distribution is first obtained by drawing a sample from the epistemic distribution of the parameter. Thus each realization from the aleatory distribution accounts for both the aleatory as well as epistemic uncertainty. The single distribution of model output thus obtained upon performing the simulation incorporates both the epistemic as well as the aleatory uncertainty [57].

Non-intrusive uncertainty propagation through simulation models is a second order or two-phase simulation. In the first phase, just like sampling based uncertainty propagation through analytic aleatory models explained in Chapters 5 through 7 , samples are drawn from the epistemic distributions of each input parameter of the aleatory (simulation) model. In the second-phase, at the set of sampled input parameter values, the simulation model is executed to obtain a distribution of the model output. $n$ invocations of the simulation model will yield $n$ such distributions which need to be interpreted to understand the contributions from aleatory and epistemic uncertainties.

The single distribution of model output resulting from intrusive propagation of 
epistemic uncertainty through simulation aleatory models is difficult to interpret as separating out the contributions from epistemic uncertainty versus aleatory uncertainty from a single distribution is not easy (this information is lost). Mixing both uncertainties in a single distribution makes it difficult to understand if the source of uncertainty in the model output is due to the epistemic uncertainty in the model input parameters or the aleatory uncertainty in the model.

As explained above, non-intrusive (two-phase simulation) uncertainty propagation separates out the aleatory and epistemic uncertainty in the model output. Each invocation of the simulation model, at a set of model input parameter values (sampled from their respective epistemic distributions) provides a distribution of model output, accounting for the aleatory uncertainty at that set of input parameter values. $n$ such curves obtained, one for each set of input parameter values sampled from their epistemic distributions, account for the epistemic uncertainty. Separating out the uncertainties helps identify how much of the total uncertainty in the model output comes from aleatory uncertainty, as compared to epistemic uncertainty. This in turn, allows identifying steps which could reduce the total uncertainty in the model output. If a large part of the total uncertainty were due to epistemic uncertainty then the uncertainty in the model output can be reduced if more information about the input parameters can be obtained (e.g. more samples from the lifetime experiments) to reduce the epistemic uncertainty. However, if a large part of total uncertainty is due to the aleatory uncertainty, the simulation model may need to be executed for a longer duration and/or the system itself may need changes [66, 57, 91],

An important consideration with the non-intrusive (two-phase simulation) approach is how to summarize and report the overall uncertainty in the model output but at the same time correctly identify the source (epistemic uncertainty or aleatory uncertainty) of total uncertainty.

Interpretation of the results of non-intrusive epistemic uncertainty propagation 
through simulation aleatory model (two-phase simulation) has been carried out in a few different ways:

- For each of the $n$ executions of the simulation model, obtain $n$ curves of the distribution of the model output. Each curve summarizes the aleatory distribution at the set of model input parameters used. Depending on the spread of the curves, determine if the major contribution to the total uncertainty is coming from epistemic or aleatory uncertainty [66, 57, 91].

- For each of the $n$ distributions obtained, compute the variance of model output. Assuming each invocation of the simulation model provides $m$ values of the model output, there will be $n$ such variances (each computed over $m$ model output values). Also compute the variance of $n \times m$ values of the model output over the course of $n$ invocations of the simulation model (one variance computed over $n \times m$ values). If the variance computed over $n \times m$ values is significantly larger than the individual $n$ variances, the uncertainty in the model output can be attributed mainly to the epistemic uncertainty and vice versa [57].

- From each of the $n$ distributions of the model output obtained by the two-phase simulation, a desired percentile point is observed. The range of values of model output at that percentile, provide the uncertainty interval, an indicator of the contribution of epistemic uncertainty to the total uncertainty in the model output, at that percentile point [73].

- Based on interval arithmetic, the confidence interval of the model output to summarize the total uncertainty in model output can be computed as : if we are interested in say 90\% 2-sided confidence interval, and $x_{5}{ }^{i}$ and $x_{95}{ }^{i}$ be the value of the model output at $5^{\text {th }}$ and $95^{\text {th }}$ percentile from the $i^{\text {th }}$ distribution respectively, then the lower limit of $90 \%$ confidence interval of model output 
summarizing both the epistemic and aleatory uncertainty can be obtained as $\min \left(x_{5}{ }^{1}, x_{5}{ }^{2}, \ldots, x_{5}{ }^{n}\right)$ and upper limit as $\max \left(x_{95}{ }^{1}, x_{95}{ }^{2}, \ldots, x_{95}{ }^{n}\right)$.

\subsection{Non-Intrusive Uncertainty Propagation}

Since the sampling based uncertainty propagation method explained in this dissertation is a non-intrusive method which acts a wrapper to already existing aleatory models and their solution techniques, the steps in uncertainty propagation through analytic aleatory models, discussed in Chapter 5 through 7 can be directly applied to propagate the epistemic uncertainty through simulation aleatory models. Latin hypercube sampling (Chapter 6) as well as introducing rank correlation between model input parameters to account for epistemic dependence 7 can be performed in case of simulation aleatory models too.

The key differences here are that in place of solving the analytic model at the parameter values sampled from the epistemic distributions, a simulation aleatory model has to be executed and that each invocation of the simulation model provides a distribution of the model output values, in place of a single value obtained by solving the analytic aleatory model.

We illustrate uncertainty propagation through a simulation aleatory model, with the help of a simple simulation model, next.

\subsubsection{Illustrative Example}

We consider availability model of a two-component system with a shared repair facility [86], as the example aleatory model to illustrate epistemic uncertainty propagation through a simulation aleatory model. The continuous time Markov chain (CTMC) availability model is shown in Figure 8.1. The system is considered to be "UP" or operational in states " 2 " and "1" (implying both or one of the components to be operational), while state " 0 " is the down state (both components failed). 


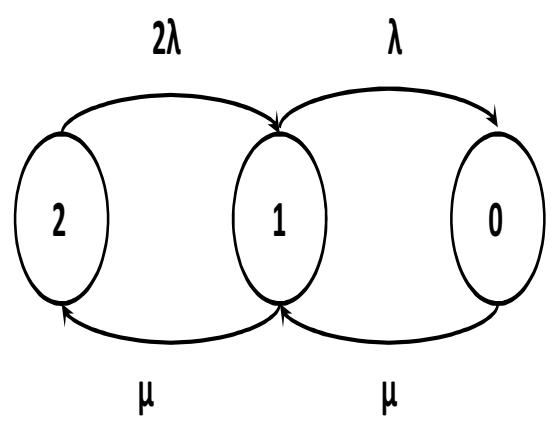

Figure 8.1: Availability Model: 2 Component System with Shared Repair

The uncertainty propagation wrapper as well as the simulation model corresponding to Figure 8.1 are implemented in Mathematica [2].

This simple aleatory model has only two input parameters, both of which are rate parameters of exponential distributions. As derived in Equation 2.14, each of the parameters has a beta epistemic distribution. The parameter values were arbitrarily chosen as $\lambda$, the failure rate of each component to be 10 per unit time and $\mu$, the repair rate to be 5 times the failure rate, and hence 50 per unit time. The epistemic uncertainty in the parameters is assumed to be presented in the form of their confidence intervals. The half-width of $95 \%$ two-sided confidence interval of input parameters is considered to be $10 \%$ of the point estimate. The number of samples $n$, to be drawn from each of the epistemic distributions is determined to 269. However, for clarity of illustration, we limit the number of samples from each 


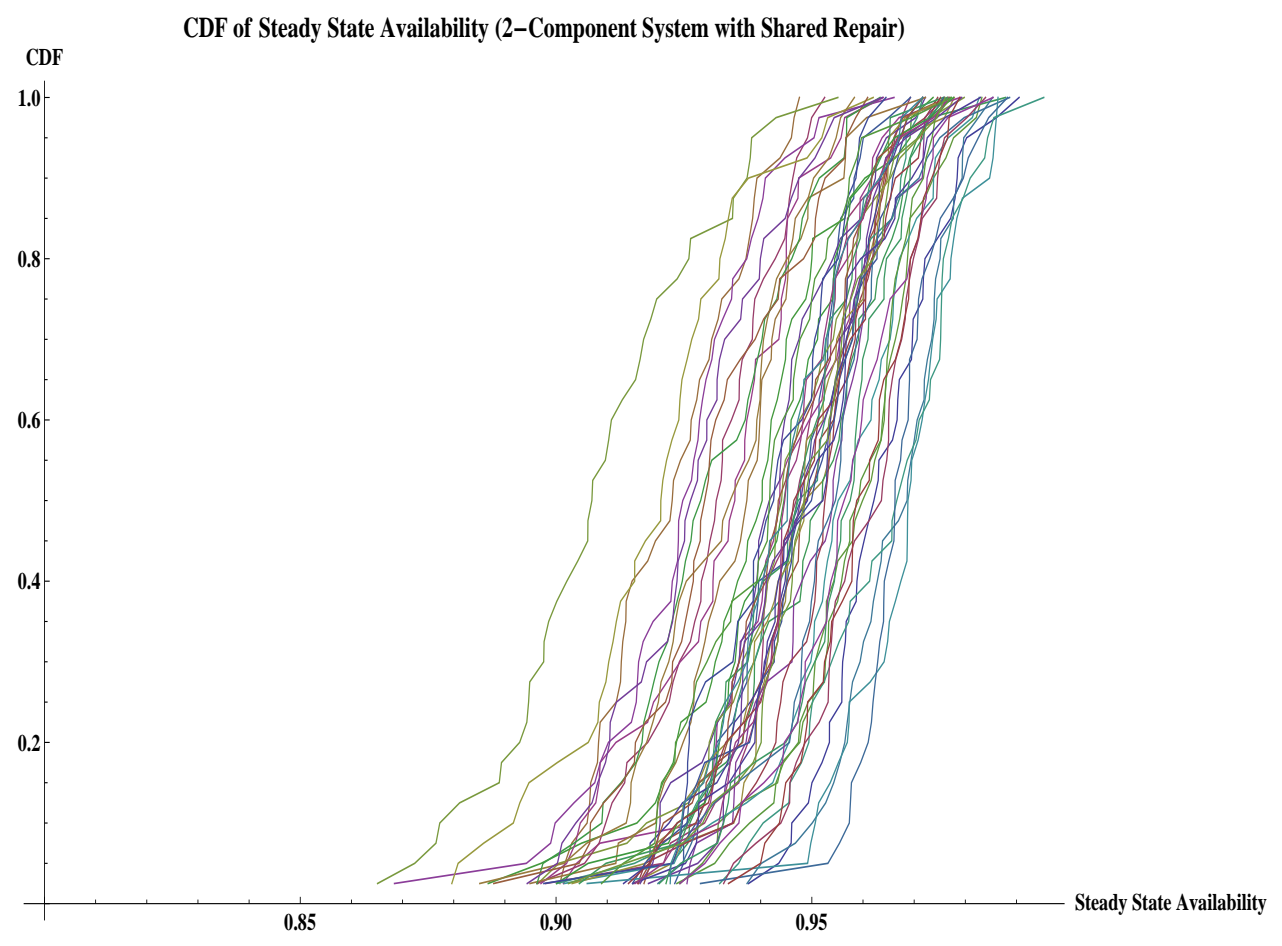

Figure 8.2: CDF: Availability of 2 Component System with Shared Repair

epistemic distribution (samples are obtained by LHS method as explained in Section 6.1) and hence the number of invocations of the simulation model to $n=50$.

Each of the invocations of the simulation model, provides a distribution of system availability, constructed out of $m=40$ values of the model output. Each of the $m$ values are obtained by running the simulation model 100 times longer than the slowest transition. Thus, there are $n=50$ distributions, each with $m=40$ values of the simulation model output. The distributions thus obtained are shown in Figure 8.2 .

As it can be seen in Figure 8.2, the $n=50$ curves are spread over a wide range of values of availability, indicating that there is a significant contribution of epistemic uncertainty in the input parameters of the aleatory model, towards the total uncertainty in the model output.

Comparing the width of $90 \%$ two-sided confidence interval of system availability, 
obtained from each of the $n=50$ distributions individually (using the value of availability at $5^{\text {th }}$ and $95^{\text {th }}$ percentile points of that distribution), to the confidence interval obtained by using the minimum value of availability at $5^{\text {th }}$ percentile point across all the $n$ distributions and maximum value of availability at $95^{\text {th }}$ percentile point across all the $n$ distributions, it can be seen that the epistemic uncertainty is a significant contributor to the total uncertainty of the model output. The width of 90\% two-sided confidence intervals thus obtained from individual distributions range from 0.02939 to 0.07211 , while the width of the $90 \%$ two-sided confidence interval obtained across all the distributions (by using the extreme values at $5^{\text {th }}$ and $95^{\text {th }}$ percentile points), is found to be 0.1134 .

The uncertainty interval at $5^{\text {th }}$ percentile point, which is the range of values of availability seen at the $5^{\text {th }}$ percentile across the $n=50$ distributions, is found to be $(0.8723,0.9531)$. Similarly, the uncertainty interval at $95^{\text {th }}$ percentile point (range of values of availability seen at the $95^{\text {th }}$ percentile across the $n=50$ distributions), is obtained as $(0.9382,0.9857)$.

\subsection{Intrusive Uncertainty Propagation}

The non-intrusive uncertainty propagation performs repeated $(n)$ executions of the simulation, each with a set of input parameter values, obtained by the sampling procedure (LHS in our case). Within each execution of the simulation in the nonintrusive method, all the samples drawn from an aleatory distribution, use the same parameter value of the aleatory distribution. In the intrusive uncertainty propagation method, for each sample to be drawn from the aleatory distribution, the parameter of the aleatory distribution is first obtained by drawing a sample from the epistemic distribution of the parameter. Thus each realization from the aleatory distribution accounts for both the aleatory as well as epistemic uncertainty. A single distribution of model output is obtained (as a result of the intrusive uncertainty propagation) 


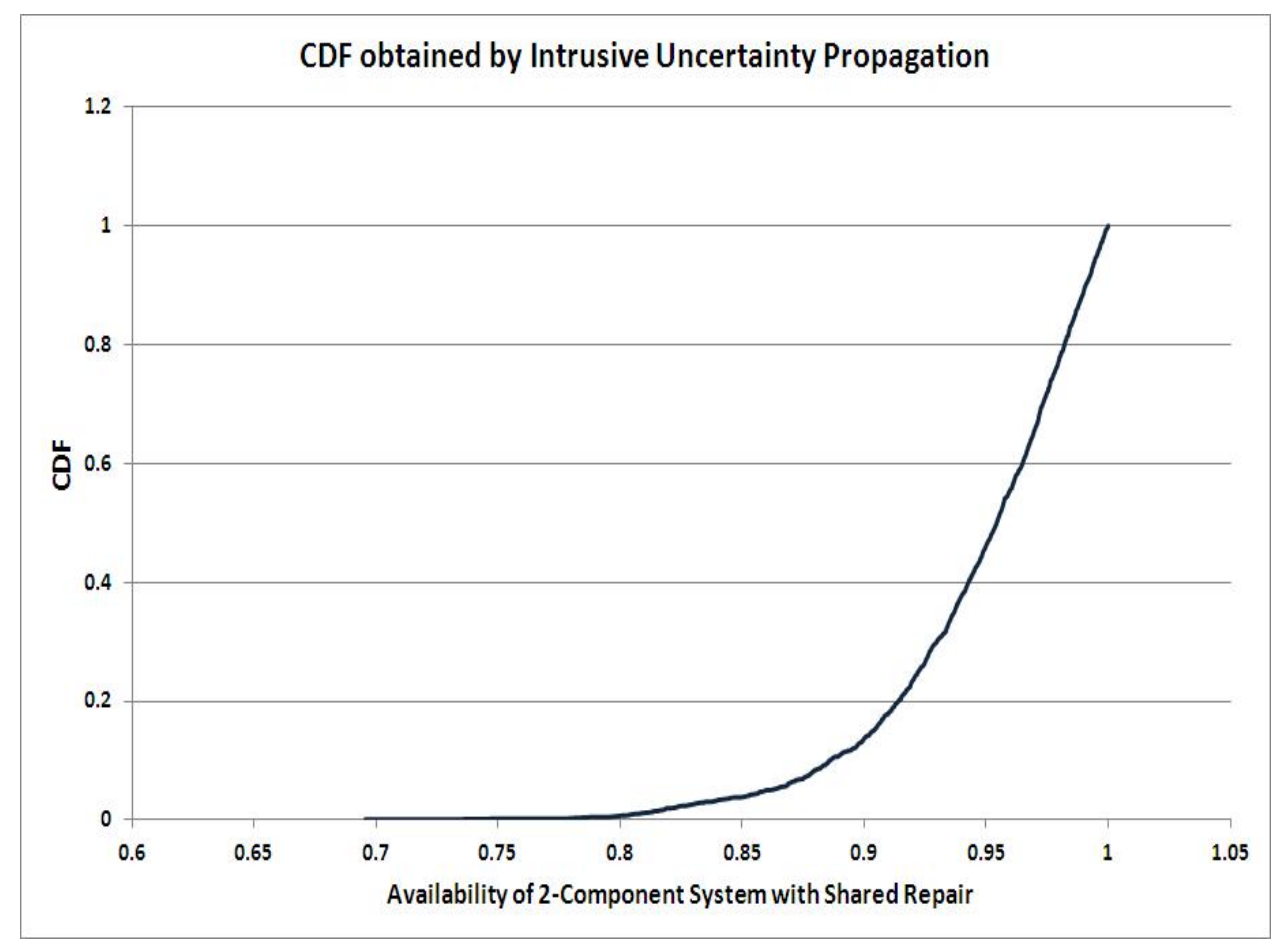

Figure 8.3: CDF of Availability by Intrusive Method

which incorporates both the epistemic and aleatory uncertainty in the model output.

We implement intrusive uncertainty propagation through the aleatory model with simulative solution, discussed in Section 8.1.1. While in the non-intrusive approach, $n=50$ distributions, each with $m=40$ values of model output were obtained, in the non-intrusive method, we obtain a single distribution with $p=2000$ values of the model output. Each of the $p$ values are obtained by running the simulation model 100 times longer than the slowest transition. Figure 8.3 provides the empirical distribution function of availability of a 2-component system with shared repair, obtained by intrusive propagation of epistemic uncertainties in the input parameters of the availability model.

Using the $5^{\text {th }}$ and $97^{\text {th }}$ percentile from empirical CDF in Figure 8.3, the $90 \%$ 2 -sided confidence interval of steady state availability of a 2 -component system with a shared repair facility, accounting for both the aleatory and epistemic uncertainties, 
is computed as $(0.79611,0.99543)$. 
9

\section{Conclusion and Future Work}

Stochastic models are often used to analyze dependability and performance of critical systems. These models are solved at fixed input parameter values to obtain the values of measures of interest. However, the model input parameters have uncertainty associated with them (known as epistemic uncertainty), as they are either derived from a finite sample size or are based on expert opinions. Solving these stochastic models at fixed parameter values, overlooks the uncertainty in the values of model output measures due to the uncertainty in the model input parameter values.

In this dissertation, computation of uncertainty in output metrics of stochastic models due to epistemic uncertainty in model input parameters was addressed, with a focus on dependability and performance models of current computer and communication systems.

An approach for propagation of parametric epistemic uncertainty through different types of stochastic aleatory models with varying complexity of models and their outputs, was presented. Uncertainty propagation was carried through a wide range of model types (ranging from simple models with closed-form expression of model output to large complex hierarchical models where only analytic-numeric solution 
is possible and even aleatory models with simulative solution), with different model output metrics. For simple aleatory models (with closed form expression of model output) the expressions for expectation, variance and distribution of model output metric due to the epistemic uncertainties, were derived and analyzed to understand their limiting behavior.

We make the following contributions in this dissertation:

- We developed an approach for epistemic uncertainty propagation through stochastic (or aleatory) models and applied it to a wide range of dependability and performance models for computer systems. These models range from simple non-state-space models with a few input parameters to large state space models or even hierarchical models with more than fifty input parameters to an aleatory model with simulative solution. A number of model output metrics were considered, including: reliability and availability of computer systems, response time of a web service, capacity oriented availability of a communication system, security (probability of successful attack) of a network routing session, expected number of jobs in a queueing system with breakdown and repair of servers and call handoff probability of a cellular wireless communication cell.

- We derived and analyzed exact closed-form expressions for distribution, expectation and variance of the model output of analytic aleatory models where the model output can be obtained as simple closed form expression of model input parameters. The results thus derived were analyzed to gain insight into the uncertainty in model output, due to the epistemic uncertainty in model input parameters and study the limiting behavior of metrics of uncertainty in model output. Expressions for distribution, expectation and variance of the model output were derived for reliability models of several simple non-repairable systems and also for availability of simple systems. 
- A numerical integration based approach was applied for epistemic uncertainty propagation through analytic aleatory models with slightly more complex closedform expression for model output.

- For uncertainty propagation through large and complex aleatory models, a sampling based non-intrusive approach was presented. This method was demonstrated to be more widely applicable than existing analytic methods and more robust (yielding more efficient estimates) than existing sampling based methods applied to epistemic uncertainty propagation through analytic dependability models of computer and communication systems. The sampling based method is also more likely to cover the entire parameter space of model input parameters, including hard to reach low probability but high impact regions (rare events).

- The uncertainty propagation approach presented can be applied when observed lifetime data of the system and its components is directly available to the modeler, as well as when only processed information about epistemic uncertainty is available, in the form of confidence intervals or bounds of the input parameters. The form and parameters of the epistemic distributions are not arbitrarily assumed, but derived based on the aleatory distribution and the provided confidence intervals. The method of obtaining the form and parameters of epistemic distribution was also presented.

- The sampling based epistemic uncertainty method was successfully applied for uncertainty propagation through several dependability and performance models of computer systems, including rather large hierarchical models with a large number of model input parameters and even a model with simulative solution.

- The sampling based method was extended to relax the assumption of epistemic 
independence (parameters of aleatory models as independent random variables) to consider correlation in the model input parameter values (epistemic dependence), even without sampling from the joint distribution function of correlated parameters. Uncertainty propagation considering epistemic dependence between input parameters was also applied to some of the dependability and performance models considered.

- The entire uncertainty propagation approach was shown to be independent of model solution tool or technique as it was applied to non-state-space, statespace, hierarchical, simulation and fixed point aleatory models solved using SHARPE, SPNP and Mathematica software packages.

As computer systems have become pervasive in an all critical aspects of modern life, accurately analyzing and predicting their dependability and performance is increasingly important. We presented the approach for quantifying the uncertainty in model outputs due to the epistemic uncertainties in their input parameters, in this dissertation. We believe this topic can be further enriched and developed in the following ways:

- While we derived the type and parameters of epistemic distributions for parameters of exponential and Bernoulli aleatory distribution, this work can be extended by deriving epistemic distributions (type and parameters) for parameters of Weibull, log normal, hypo-exponential and other aleatory distributions commonly seen in dependability and performance models of computer systems. Deriving epistemic distributions for parameters of these other aleatory distributions will enable the uncertainty propagation approach discussed in this dissertation to be applied to a wider range of aleatory models (e.g. semi-Markov process and Markov regenerative process models) which in some cases may be a closer representation of the system behavior being modeled. 
- While we applied the numerical integration based approach and closed-form analytic approach on several examples, a systematic classification of aleatory model types based on applicability of analytic closed-form and numerical integration approaches needs to be performed. Properties of the aleatory model and/or its output expression limiting the applicability of either of these methods need to be identified.

- Epistemic independence was considered in the sampling based uncertainty propagation method. Since the closed-form analytic approach provides exact expressions for measures of uncertainty of the model output, exploring and developing ways of incorporating epistemic dependence in the closed form epistemic uncertainty propagation approach, will make it even more accurate.

- To incorporate epistemic dependence in the sampling based method, we consider rank correlation between dependent parameters. Ways to consider other measures of dependence like Pearson's product moment correlation need to be explored.

- The computation time in each of these approaches need to be understood. 


\section{Appendix A}

\section{Mathematica, SHARPE and SPNP Input Files}

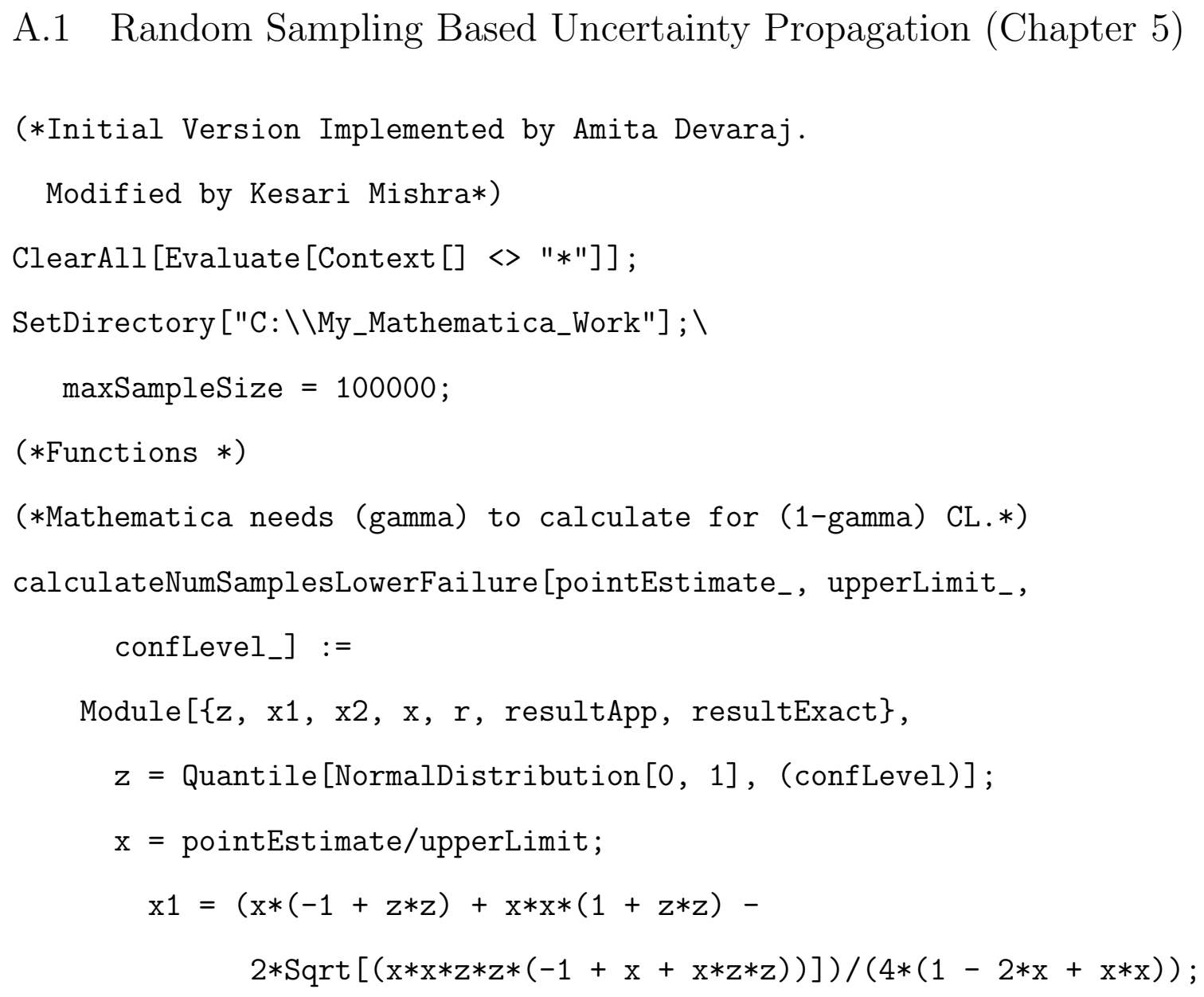




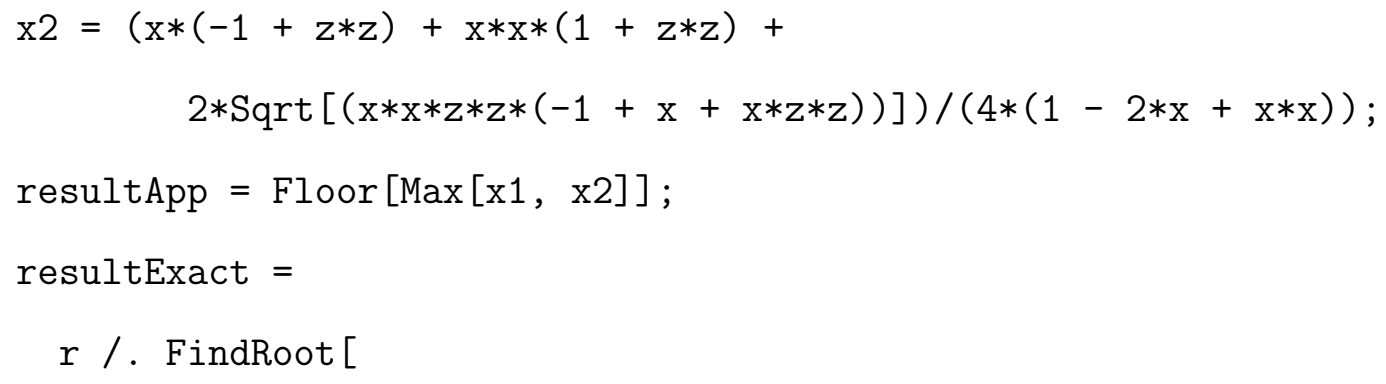

InverseCDF [ChiSquareDistribution $[2 r],(1-\operatorname{confLevel})] * \mathrm{x} / 2==$ $r,\{r$, resultApp/2, resultApp $* 2\}$, Method $\rightarrow$ Brent] ; resultExact $=$ Floor $[$ resultExact $]$; 


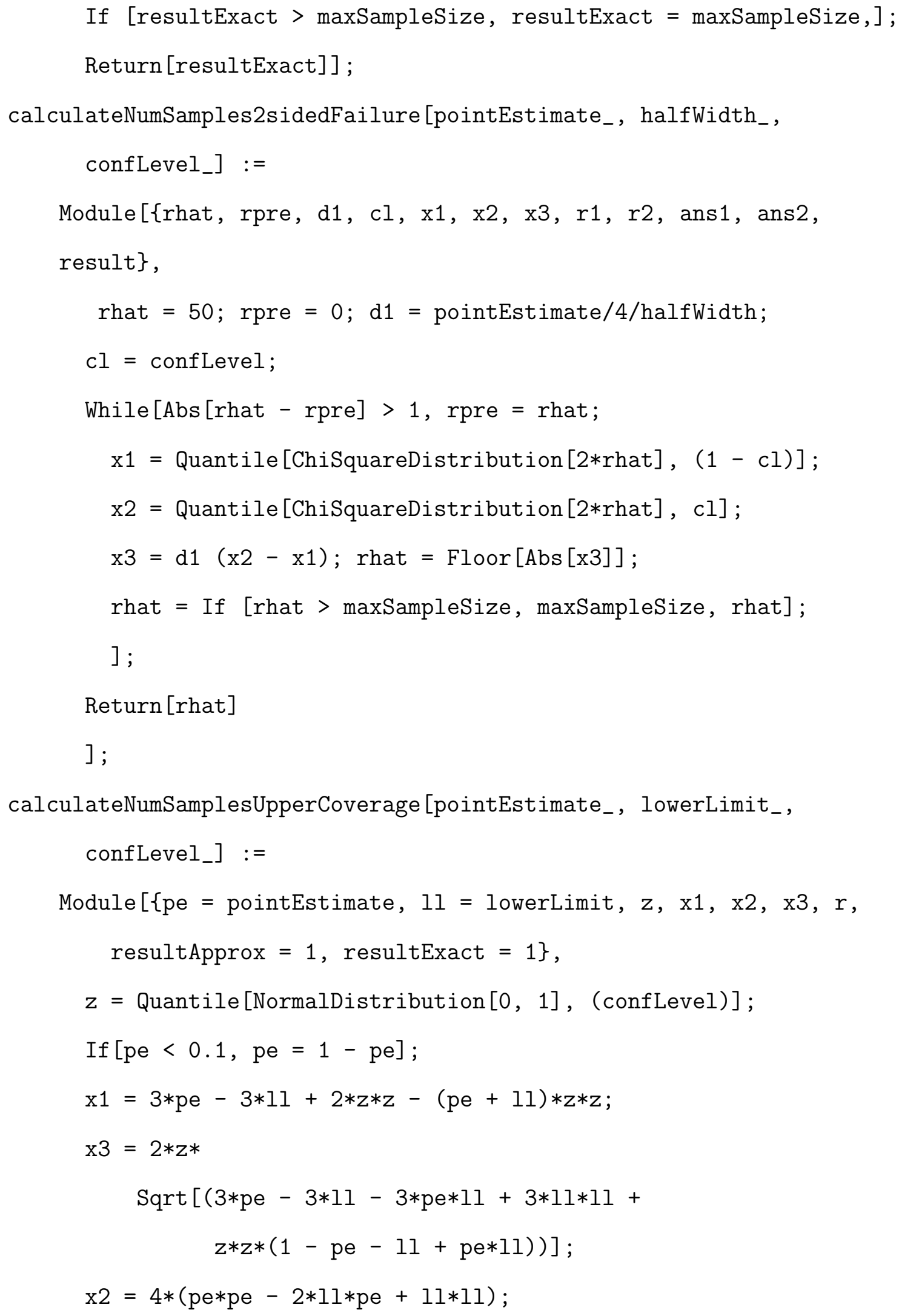




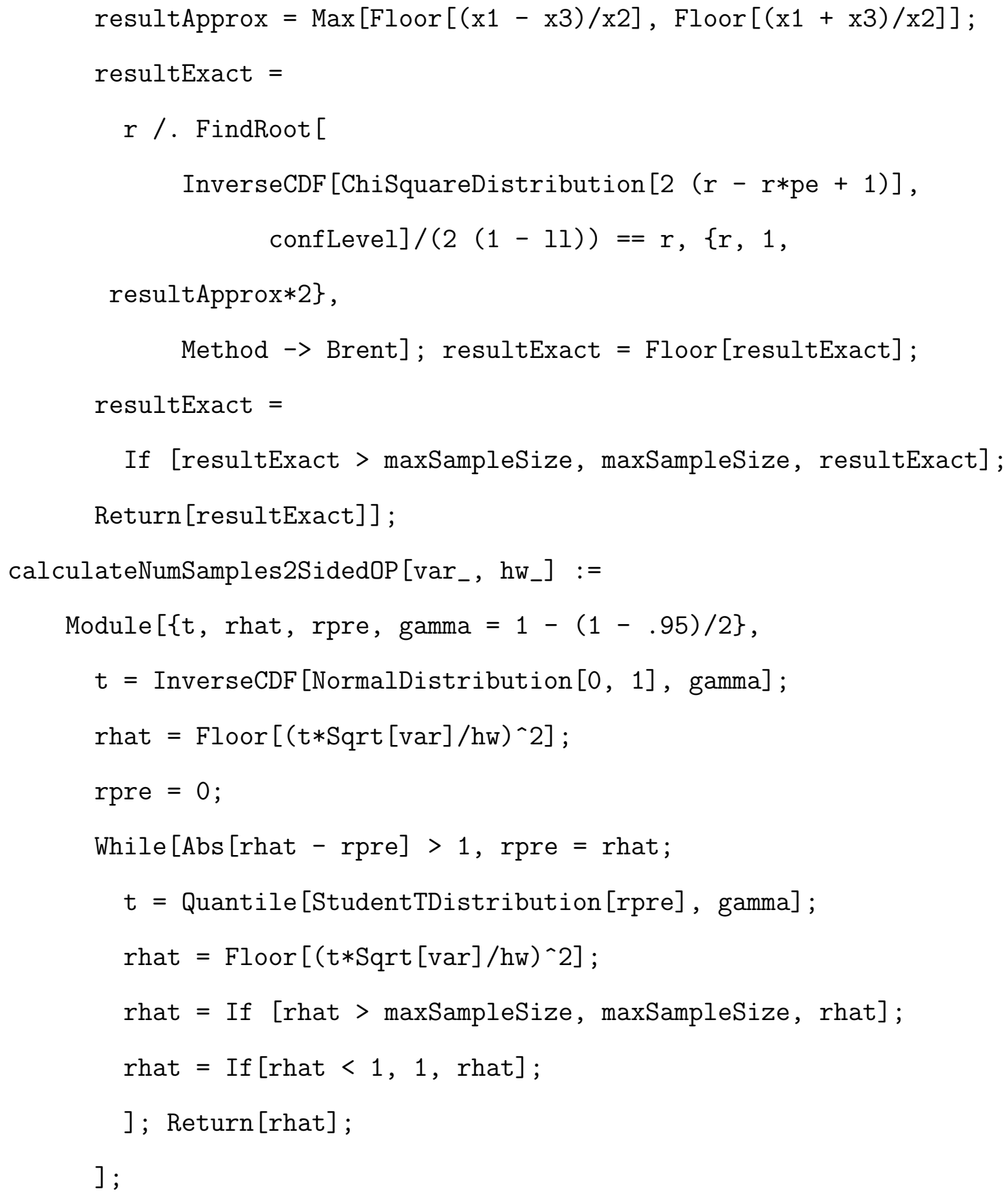




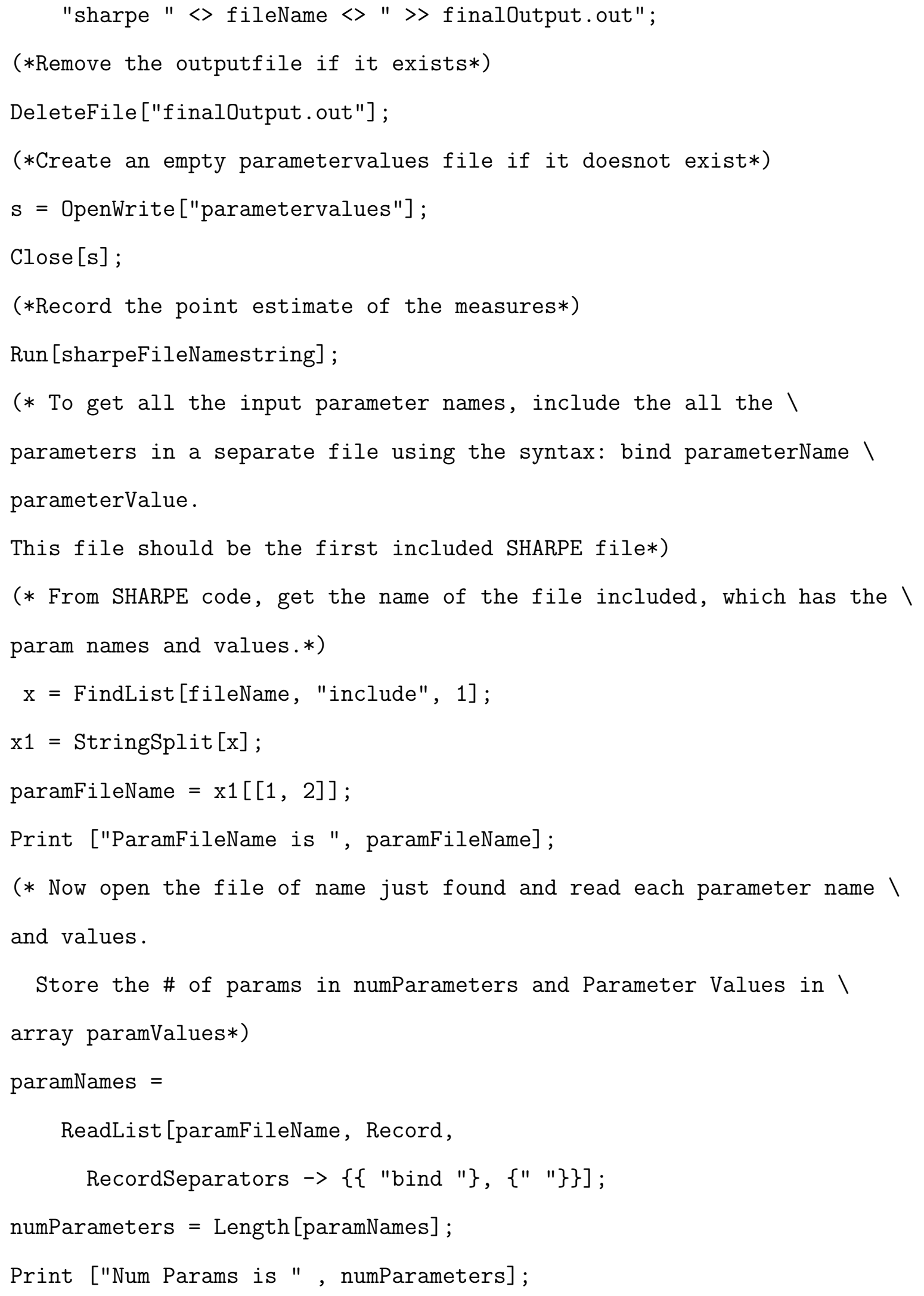




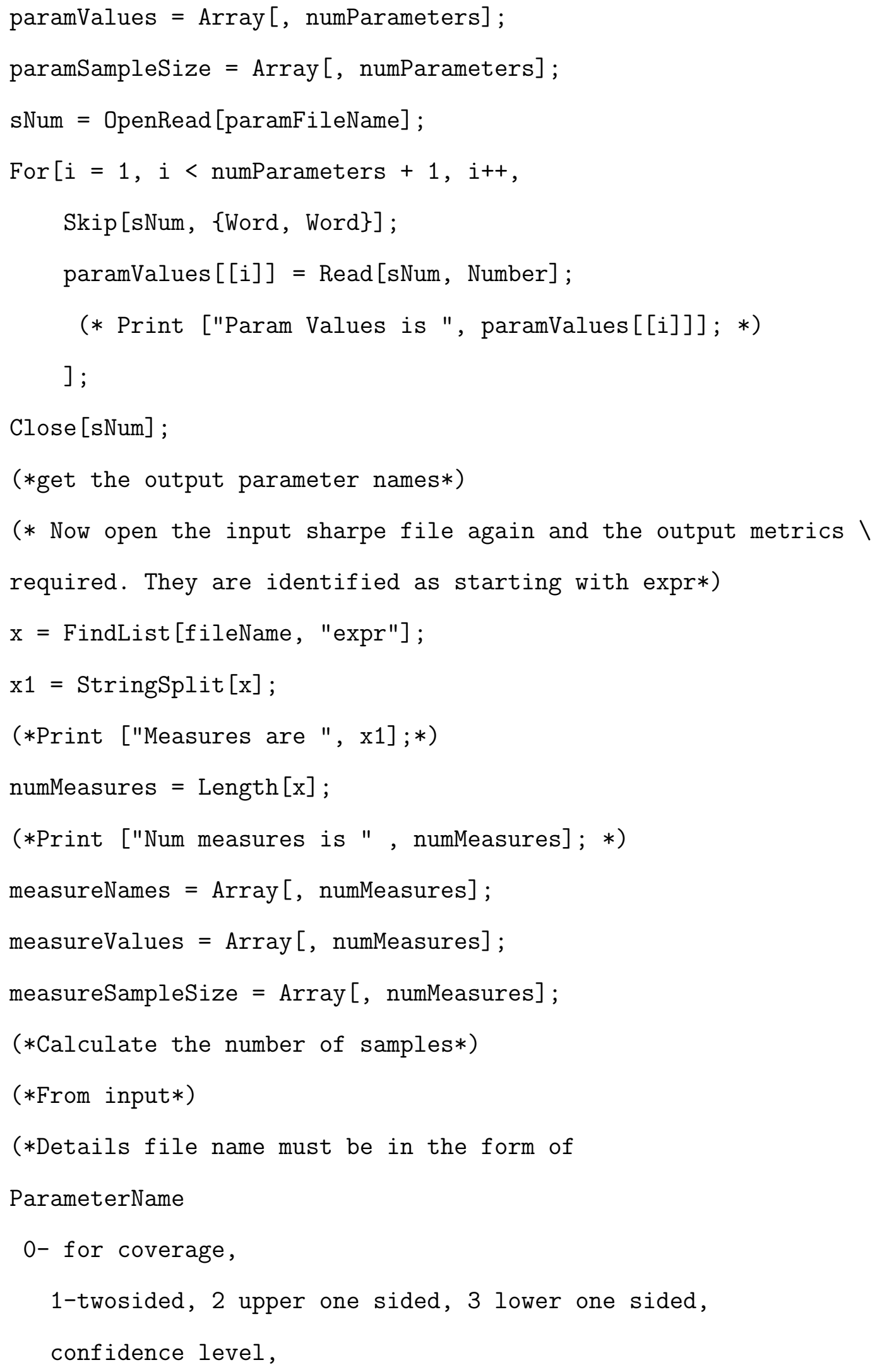


half width or lower or upper limit*)

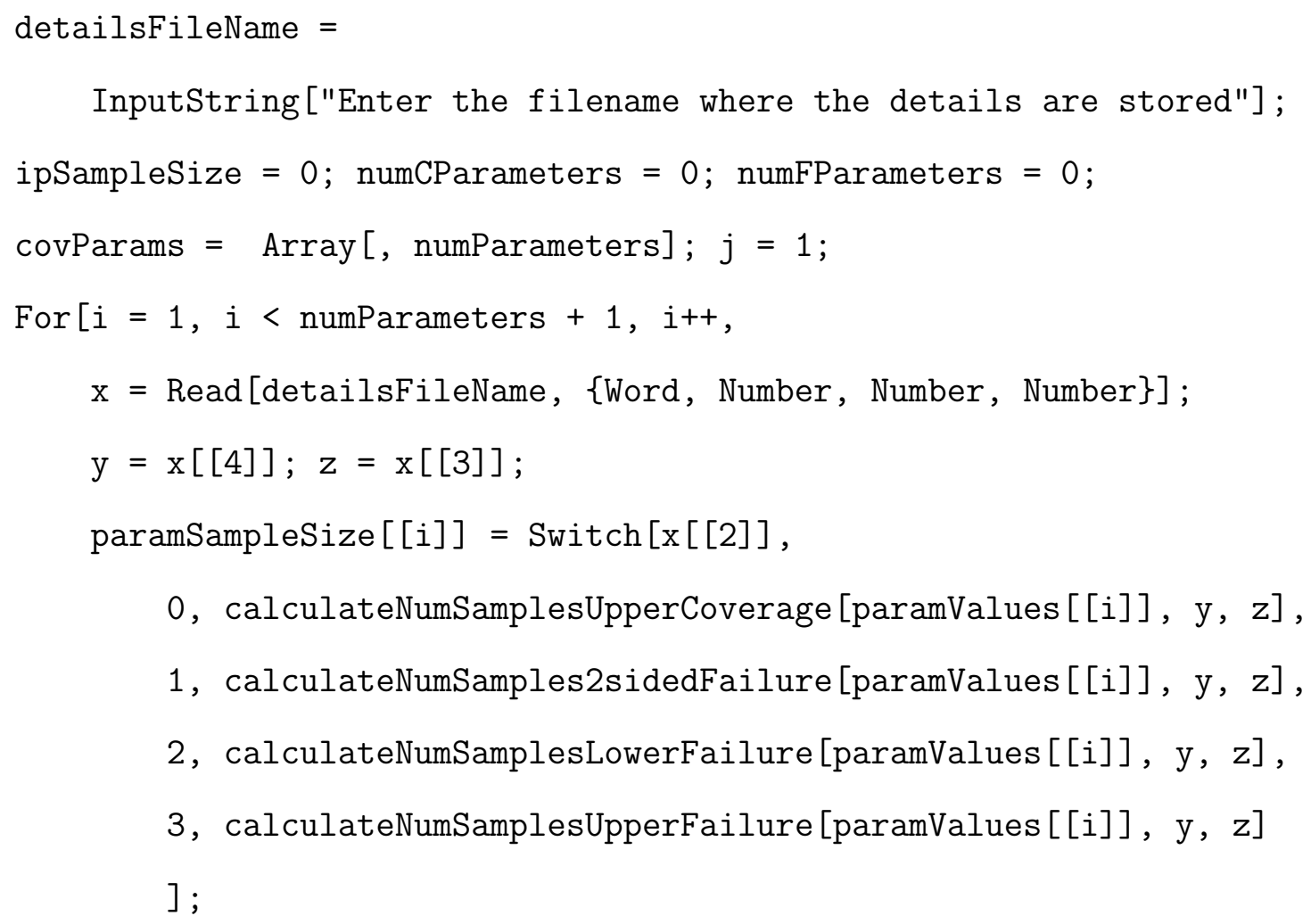

(* Identify which parameters are coverage parameters by storing the \ value $i, i . e$. the parameter number in array, covParams.

Also keep a counter to track how many parameters are coverage $\backslash$ parameters. *)

$\operatorname{If}[x[[2]]==0$, numCParameters++; $\operatorname{covParams}[[j]]=i ; j++$;

(* Print ["This is a coverage Parameter"]; *)

];

(* Get a running max of number of smaples from input across all । input params $*$ )

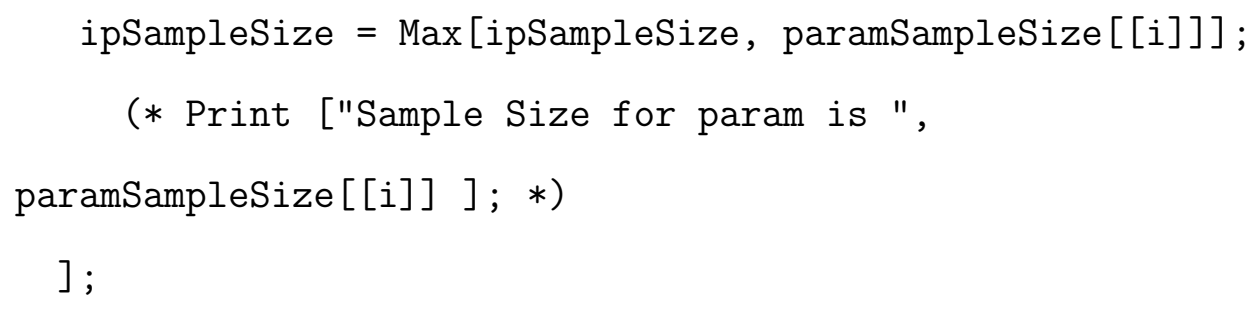


(* Number of failure parameters are numFParameters and coverage are \ numCParameters *)

numFParameters $=$ numParameters - numCParameters;

(*Print ["Input Sample Size is ", ipSampleSize];

Print ["Num Failure Params and Coverage Params are ", numFParameters, । numCParameters] ; *)

Close [detailsFileName] ;

(* Since now we know which params are coverage, Store the coverage \ parameter names, values and num samples in different arrays*)

$\mathrm{j}=1 ; \mathrm{k}=1$;

paramCNames = Array [, numCParameters $]$;

paramFNames = Array [, numFParameters $]$;

paramCValues = Array $[$, numCParameters $]$;

paramFValues = Array $[$, numFParameters $]$;

paramCSampleSize = Array [, numCParameters $]$;

paramFSampleSize = Array [, numFParameters $]$;

For $[i=1, i<$ numParameters $+1, i++$,

$\operatorname{If}[\operatorname{covParams}[[j]]==i, \operatorname{paramCNames}[[j]]=\operatorname{paramNames}[[i]]$;

(* Print ["ith parameter is the $j$ th coverage parameter $i j$ ", $i, j] ; *)$

paramCValues $[[j]]=$ paramValues $[[i]]$;

paramCSampleSize $[[j]]$ = paramSampleSize $[[i]]$;

(* Print ["Sample Size for jth coevrage parameter is ", paramCSampleSize $[[j]]] ; *)$

j++; ,

(*Print ["ith parameter is the kth failure parameter $i k$ ", i, $\mathrm{k}] ; *$ ) 


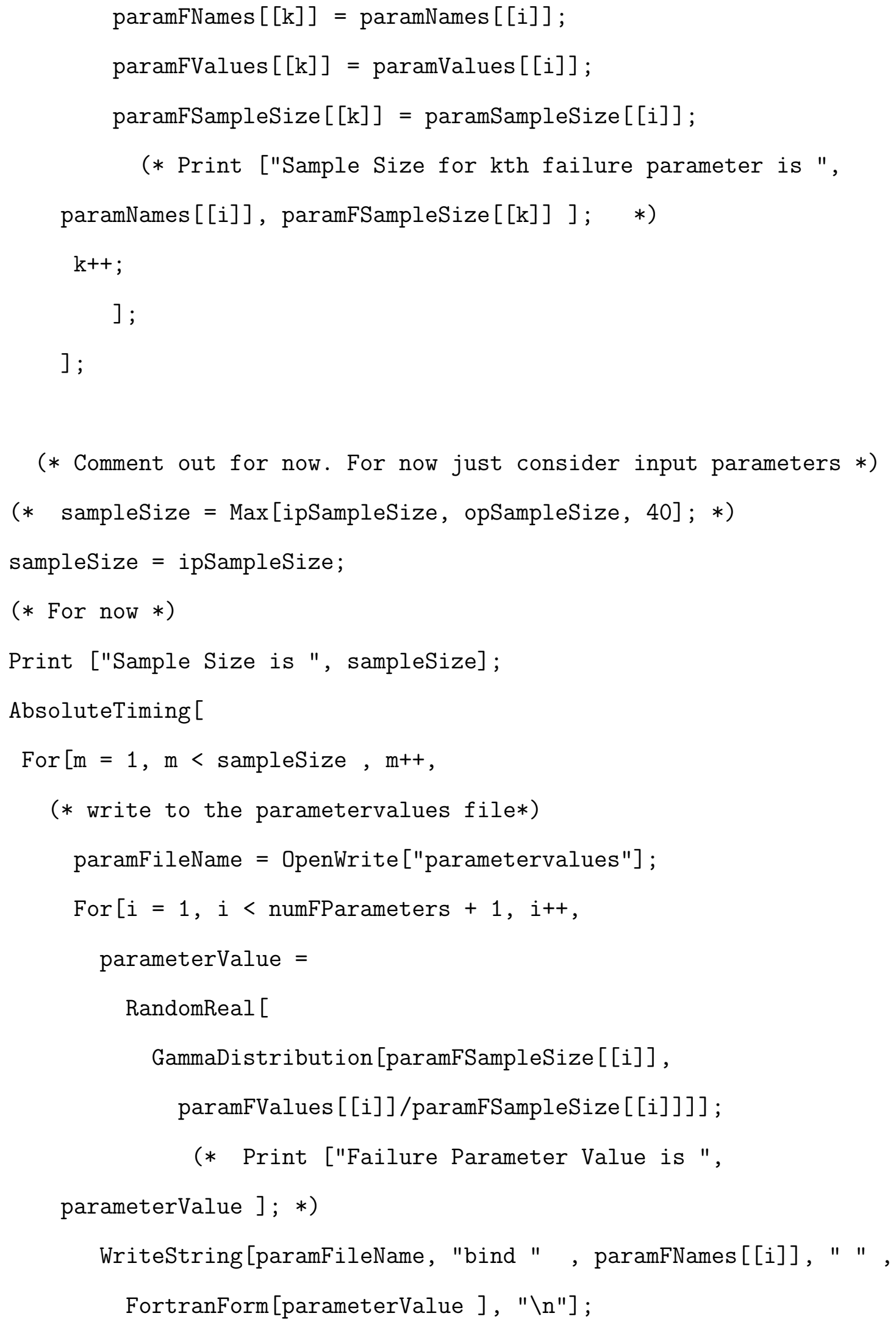




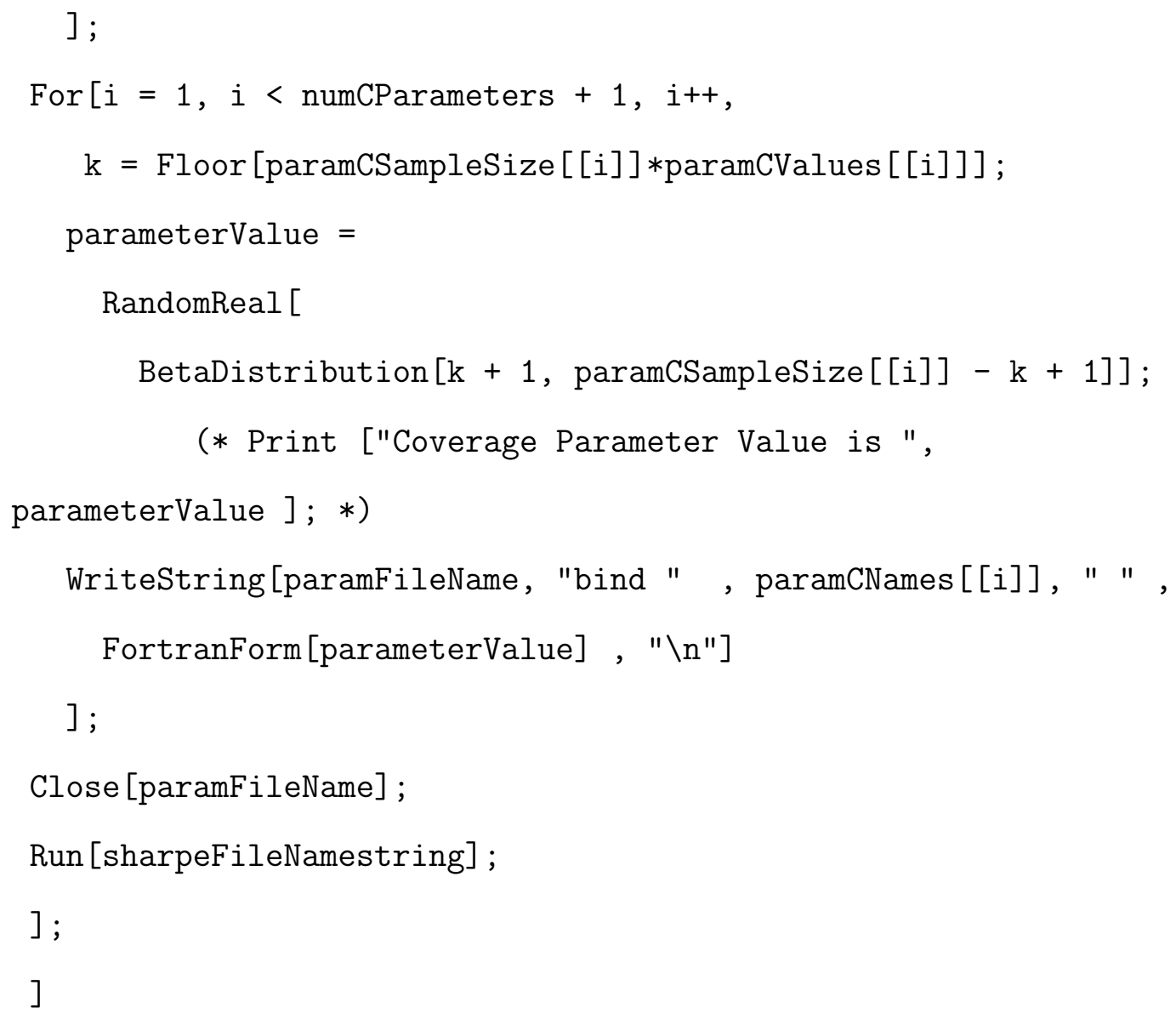

\section{A.2 LHS Based Uncertainty Propagation (Chapter 6)}

(* The part determining number of observations, implemented by Amita Devaraj*)

ClearAll [Evaluate[Context [] <> "*"]];

SetDirectory ["C: \\My_Mathematica_Work"] ;

maxSampleSize $=100000 ;$

calculateNumSamplesLowerFailure [pointEstimate_, upperLimit_, confLevel_] := Module $[\{z, x 1, x 2, x, r$, resultApp, resultExact $\}$, $z=$ Quantile[NormalDistribution $[0,1],($ confLevel)]; $\mathrm{x}=$ pointEstimate/upperLimit; 


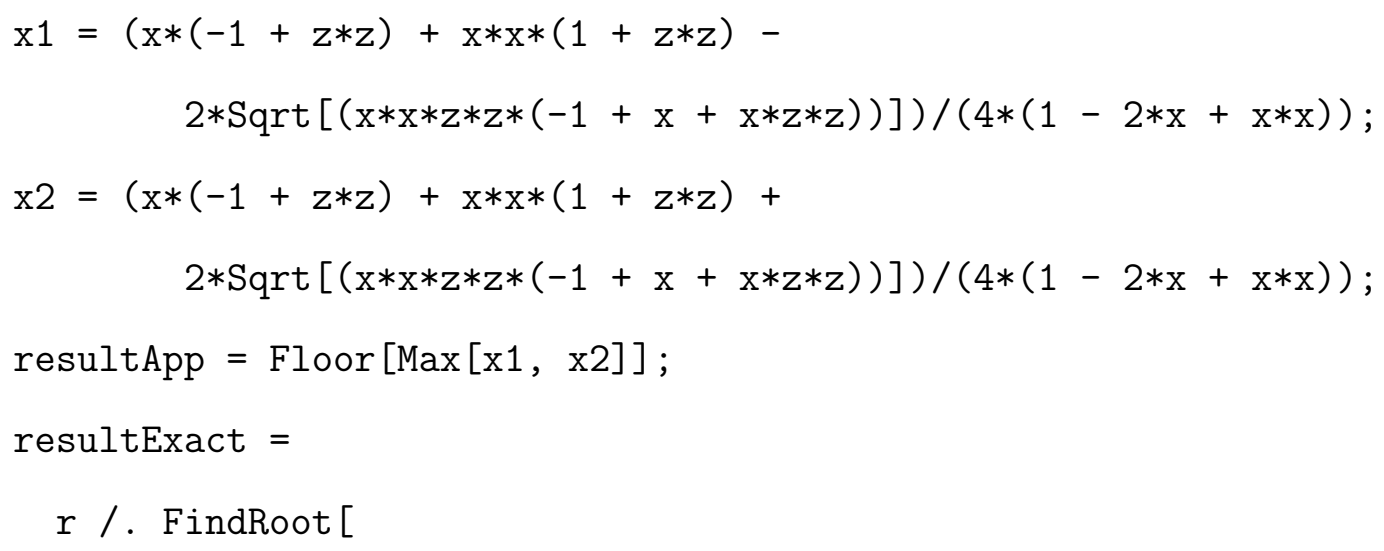

InverseCDF [ChiSquareDistribution[2 $r]$, confLevel] $* x / 2==r,\{r$, resultApp/2, resultApp*2\}, Method $\rightarrow$ Brent];

resultExact $=$ Floor [resultExact $] ;(*$ To Do: If $[$ resultExact $<$

\section{1] ; *$,}

If $[$ resultExact $>$ maxSampleSize, resultExact $=$ maxSampleSize,]; Return [resultExact] ];

calculateNumSamplesUpperFailure [pointEstimate_, lowerLimit_, confLevel_] := Module $[\{z, x 1, x 2, x, r, r e s u l t A p p, r e s u l t E x a c t\}$, $z=$ Quantile [NormalDistribution $[0,1],($ confLevel)]; $\mathrm{x}=$ pointEstimate/lowerLimit; $\mathrm{x} 1=(\mathrm{x} *(-1+\mathrm{z} * \mathrm{z})+\mathrm{x} * \mathrm{x} *(1+\mathrm{z} * \mathrm{z})-$ $2 * \operatorname{Sqrt}[(\mathrm{x} * \mathrm{x} * \mathrm{z} * \mathrm{z} *(\mathrm{x}-1+\mathrm{x} * \mathrm{z} * \mathrm{z}))]) /(4 *(\mathrm{x}-1) *(\mathrm{x}-1)) ;$ $\mathrm{x} 2=(\mathrm{x} *(-1+\mathrm{z} * \mathrm{z})+\mathrm{x} * \mathrm{x} *(1+\mathrm{z} * \mathrm{z})+$ $2 * \operatorname{Sqrt}[(\mathrm{x} * \mathrm{x} * \mathrm{z} * \mathrm{z} *(\mathrm{x}-1+\mathrm{x} * \mathrm{z} * \mathrm{z}))]) /(4 *(\mathrm{x}-1) *(\mathrm{x}-1))$; resultApp $=$ Floor $[\operatorname{Max}[\mathrm{x} 1, \mathrm{x} 2]]$; resultExact $=$ $r /$. FindRoot [ 
InverseCDF [ChiSquareDistribution[2 r], (1 - confLevel)] $* x / 2==$ $r,\{r$, resultApp/2, resultApp*2\}, Method $\rightarrow$ Brent] ; resultExact $=$ Floor $[$ resultExact $] ;(*$ To Do: If $[$ resultExact $<$ 1] ; *$,

If [resultExact $>$ maxSampleSize, resultExact $=$ maxSampleSize,]; Return [resultExact] ];

calculateNumSamples2sidedFailure [pointEstimate, halfWidth_, confLevel_] := Module $[\{r h a t$, rpre, $d 1, \mathrm{cl}, \mathrm{x} 1, \mathrm{x} 2, \mathrm{x} 3, \mathrm{r} 1, \mathrm{r} 2$, ans1, ans2, result\},

rhat $=50 ;$ rpre $=0 ; \mathrm{d} 1=$ pointEstimate $/ 4 /$ halfWidth;

cl = confLevel;

While [Abs [rhat - rpre] > 1, rpre = rhat;

$\mathrm{x} 1=$ Quantile [ChiSquareDistribution[2*rhat], $(1-\mathrm{cl})]$;

$\mathrm{x} 2=$ Quantile[ChiSquareDistribution [2*rhat], cl];

$\mathrm{x} 3=\mathrm{d} 1(\mathrm{x} 2-\mathrm{x} 1) ;$ rhat $=$ Floor $[\mathrm{Abs}[\mathrm{x} 3]]$;

rhat $=$ If $[$ rhat $>$ maxSampleSize, maxSampleSize, rhat $]$;

];

Return [rhat]

] ;

calculateNumSamplesUpperCoverage [pointEstimate_, lowerLimit_, confLevel_] :=

Module $[\{$ pe $=$ pointEstimate, $11=$ lowerLimit, $z, x 1, x 2, x 3, r$, resultApprox $=1$, resultExact $=1\}$, If $[\mathrm{pe}<0.1$, pe $=1-$ pointEstimate $]$; $z=$ Quantile[NormalDistribution $[0,1]$, (confLevel)]; $\mathrm{x} 1=3 *$ pe $-3 * 11+2 * z * z-($ pe +11$) * z * z ;$ 


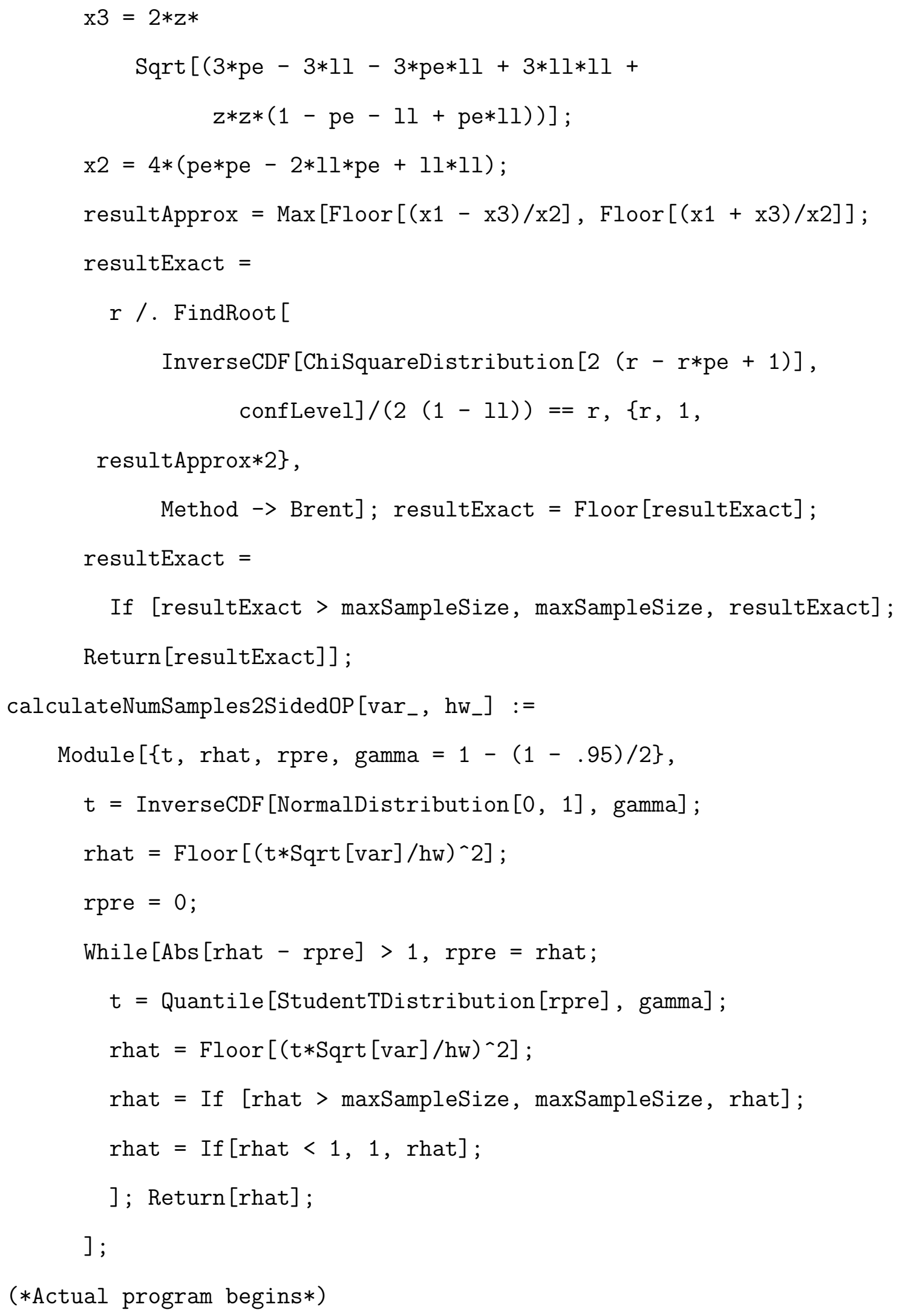


fileName = InputString["Enter the SHARPE Input file name"];

(*Construct string to run sharpe*)

sharpeFileNamestring $=$

"sharpe " <> fileName <> " >> finalOutput.out";

(*Remove the outputfile if it exists*)

DeleteFile ["finalOutput.out"];

(*Create an empty parametervalues file if it doesnot exist*)

s = OpenWrite ["parametervalues"];

Close $[\mathrm{s}]$;

(*Record the point estimate of the measures*)

Run [sharpeFileNamestring] ;

(* To get all the input parameter names, include the all the $\backslash$

parameters in a separate file using the syntax: bind parameterName \

parameterValue.

This file should be the first included SHARPE file*)

(* From SHARPE code, get the name of the file included, which has the param names and values.*)

$\mathrm{x}=$ FindList $[\mathrm{fileName,} \mathrm{"include",} \mathrm{1];}$

$\mathrm{x} 1=$ StringSplit $[\mathrm{x}]$;

paramFileName $=\mathrm{x} 1[[1,2]]$;

(*Print ["ParamFileName is ", paramFileName];*)

(* Now open the file of name just found and read each parameter name । and values.

Store the \# of params in numParameters and Parameter Values in $\backslash$ array paramValues*)

paramNames $=$ 


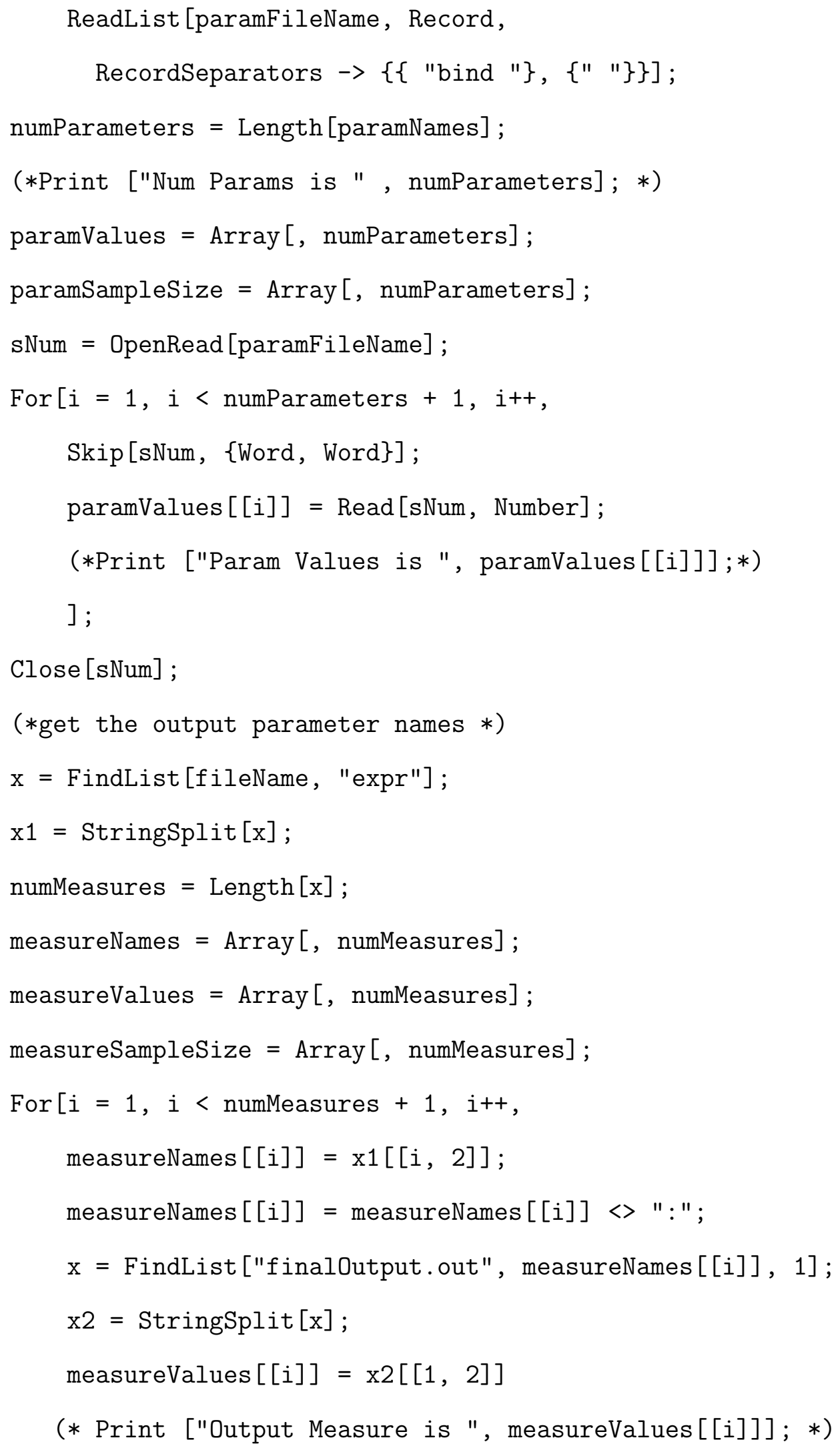


(*Calculate the number of samples*)

(*Details file name must be in the form of

ParameterName

0- for coverage,

1-twosided, 2 upper one sided, 3 lower one sided, confidence level, half width or lower or upper limit*) detailsFileName $=$

InputString["Enter the filename where the details are stored"]; ipSampleSize $=0 ;$ numCParameters $=0 ;$ numFParameters $=0 ; \operatorname{covParams}=$ Array [, numParameters $] ; j=1$;

For $[i=1, i<$ numParameters $+1, i++$, $\mathrm{x}=\operatorname{Read}[$ detailsFileName, \{Word, Number, Number, Number\}]; $\mathrm{y}=\mathrm{x}[[4]] ; \mathrm{z}=\mathrm{x}[[3]]$; paramSampleSize $[[i]]=\operatorname{Switch}[\mathrm{x}[[2]]$, 0, calculateNumSamplesUpperCoverage [paramValues [[i]], y, z], 1, calculateNumSamples2sidedFailure [paramValues [[i]], y, z], 2, calculateNumSamplesLowerFailure[paramValues [[i]], y, z], 3, calculateNumSamplesUpperFailure[paramValues [[i]], y, z] ]; $\operatorname{If}[x[[2]]==0$, numCParameters $++; \operatorname{covParams}[[j]]=i ; j++$; ] ; ipSampleSize = Max [ipSampleSize, paramSampleSize[[i]]]; ] ;

numFParameters $=$ numParameters - numCParameters; Close [detailsFileName] ; 


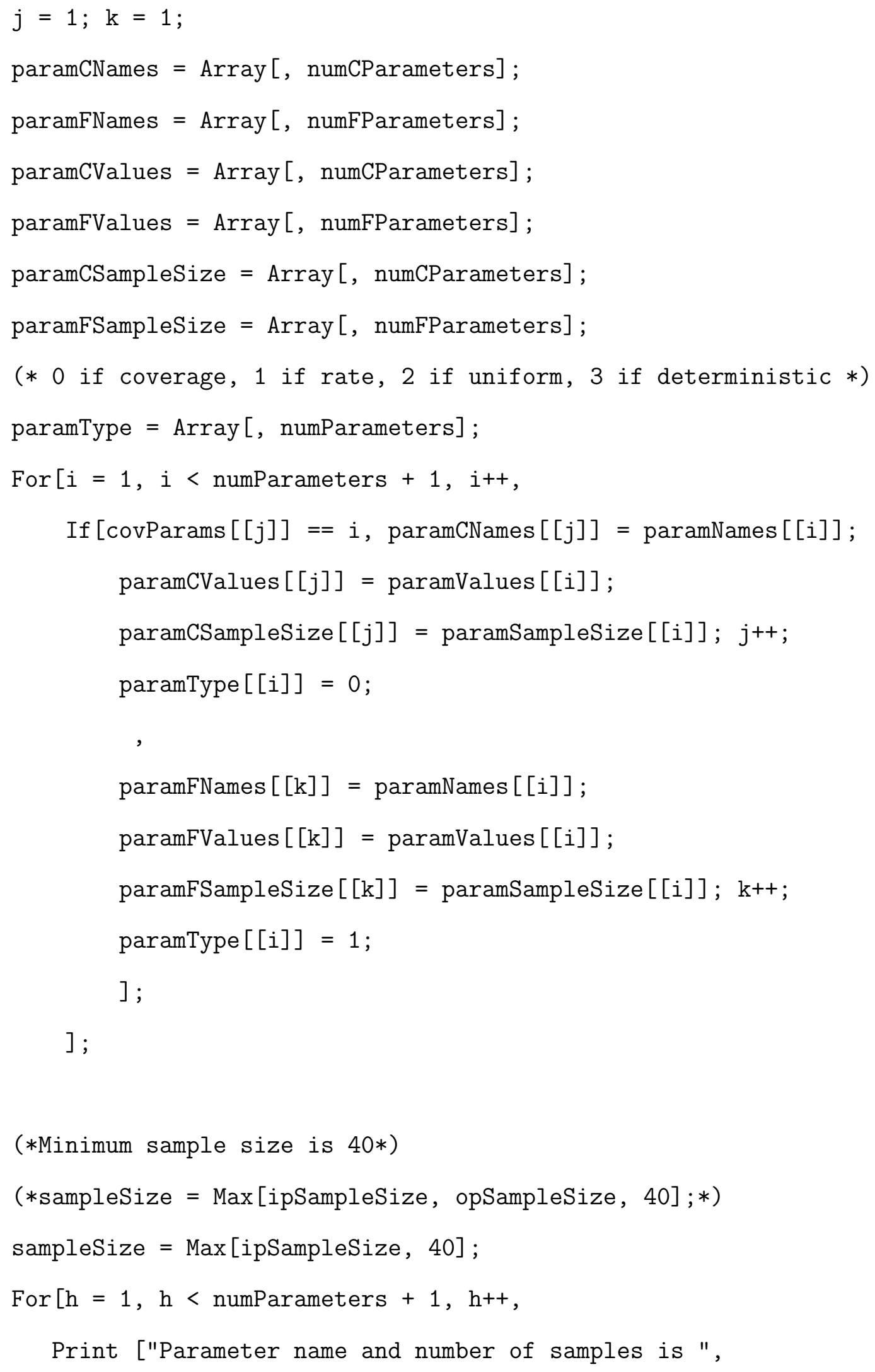




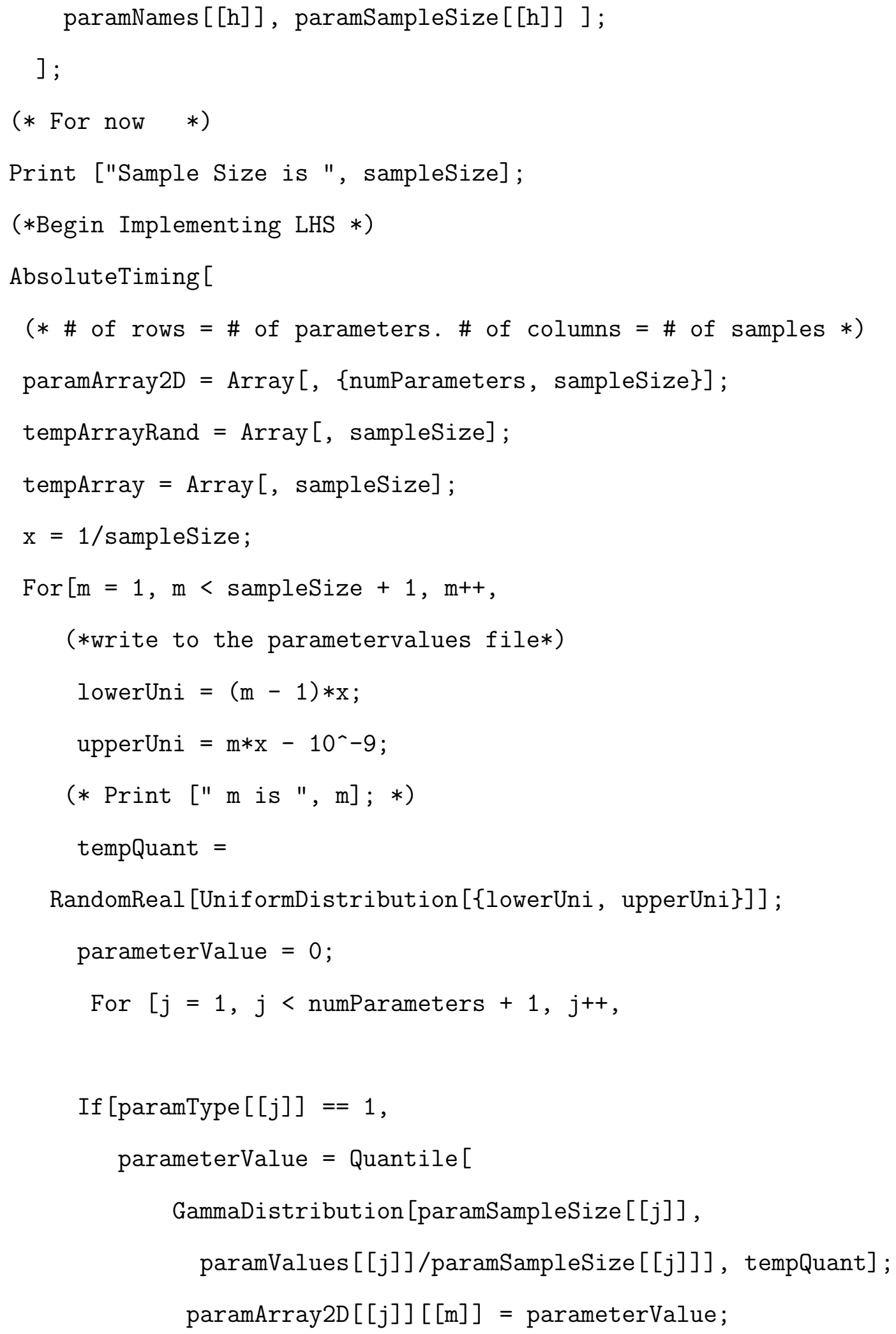




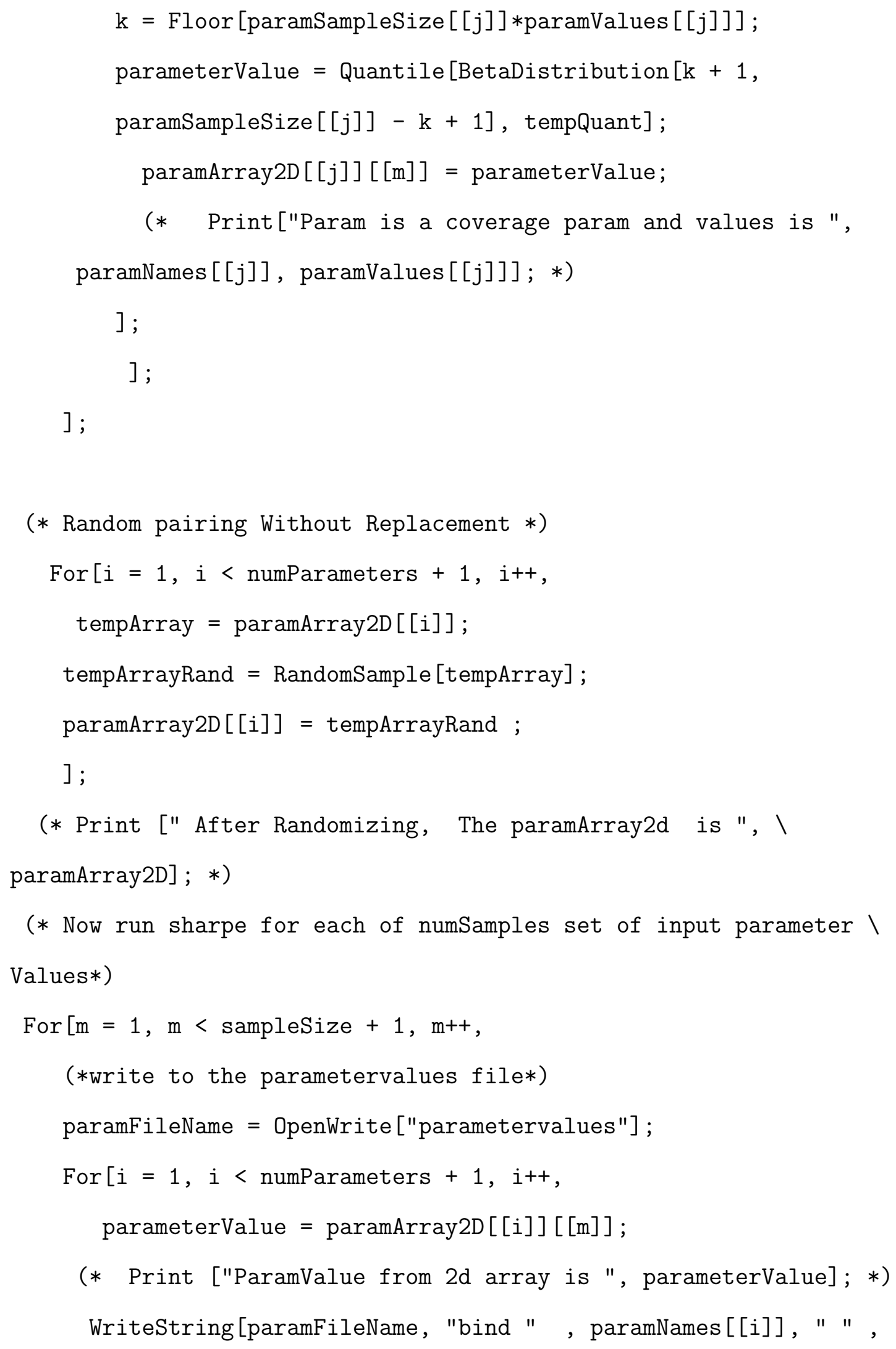




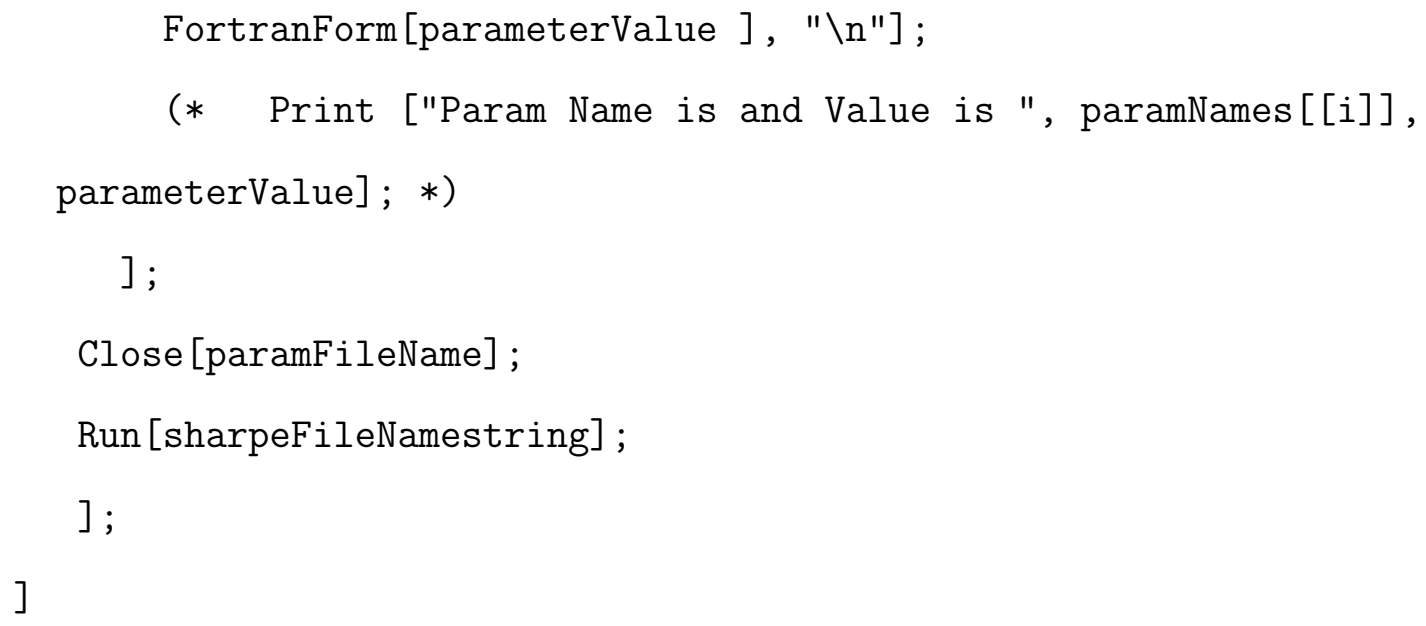

A.3 SHARPE Input File for Section 6.2.2

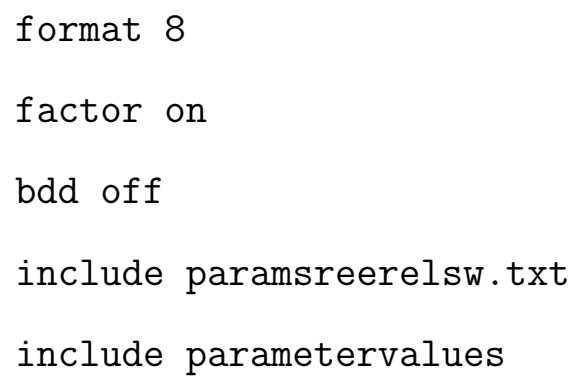


bind

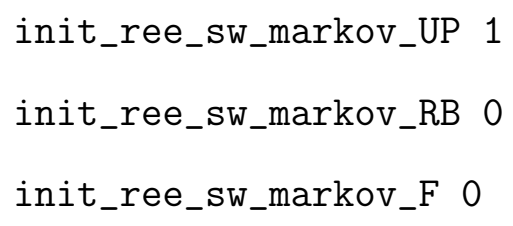




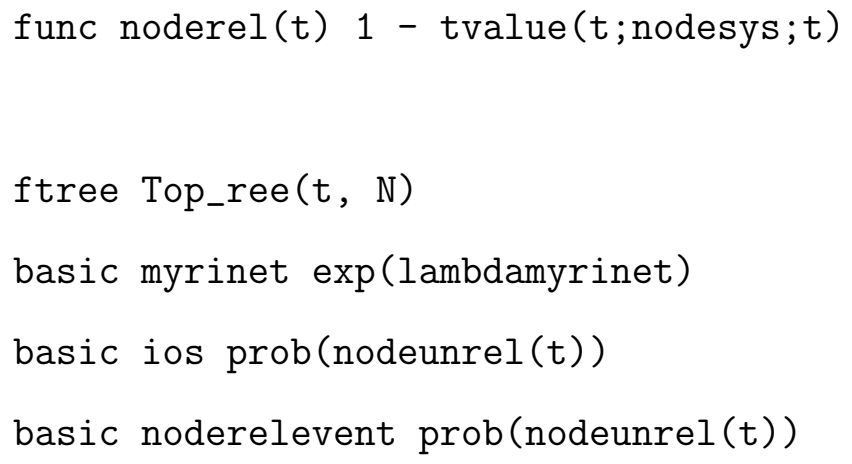

end

\section{A.4 SPNP Input File for Section 6.2.4}

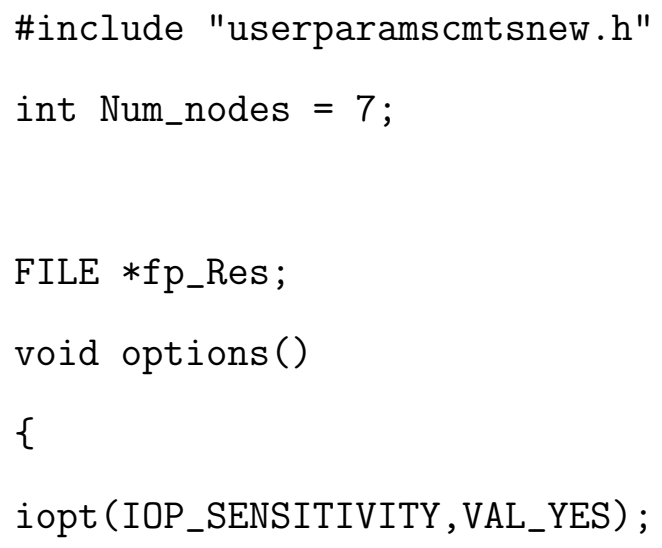




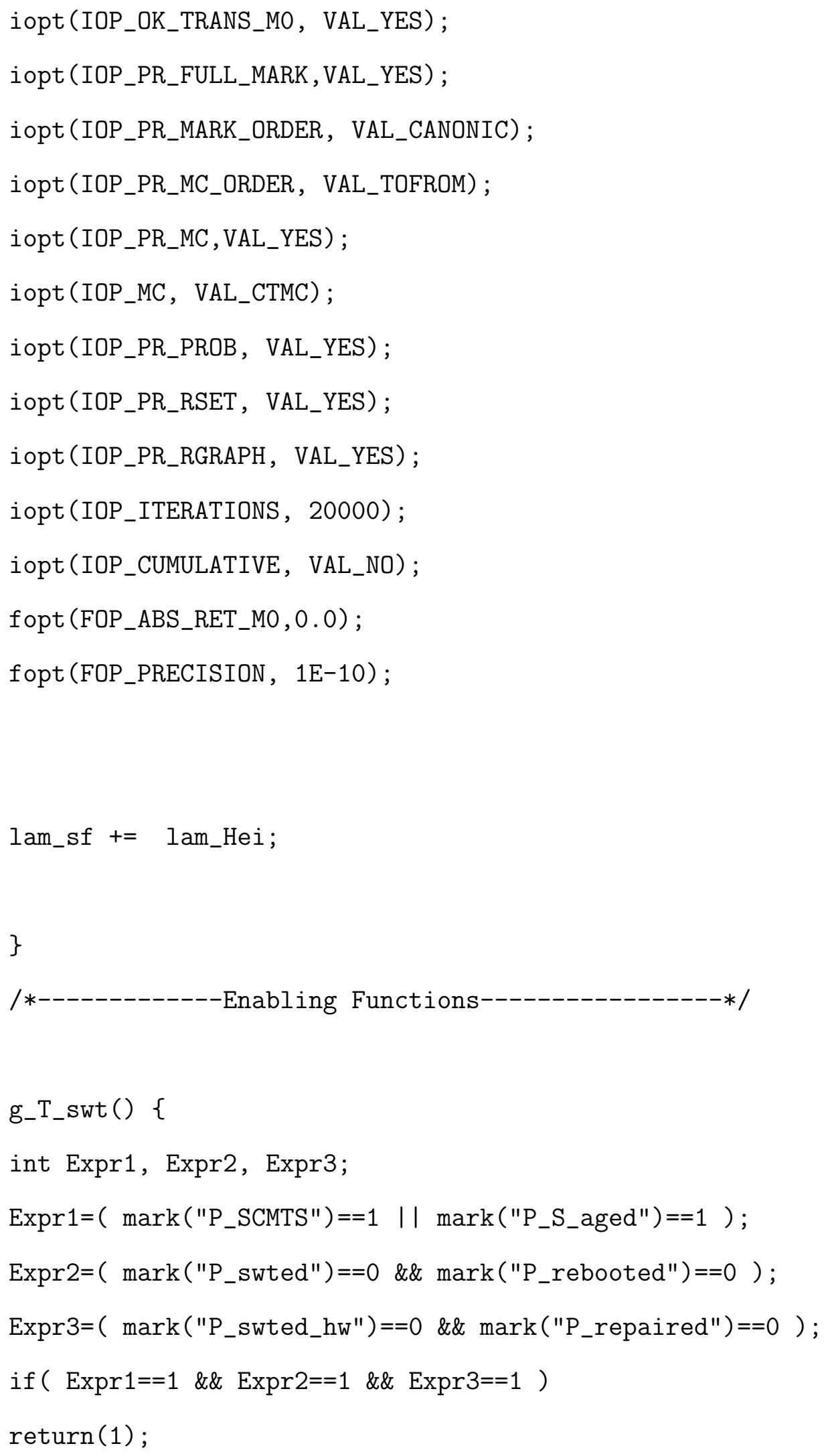




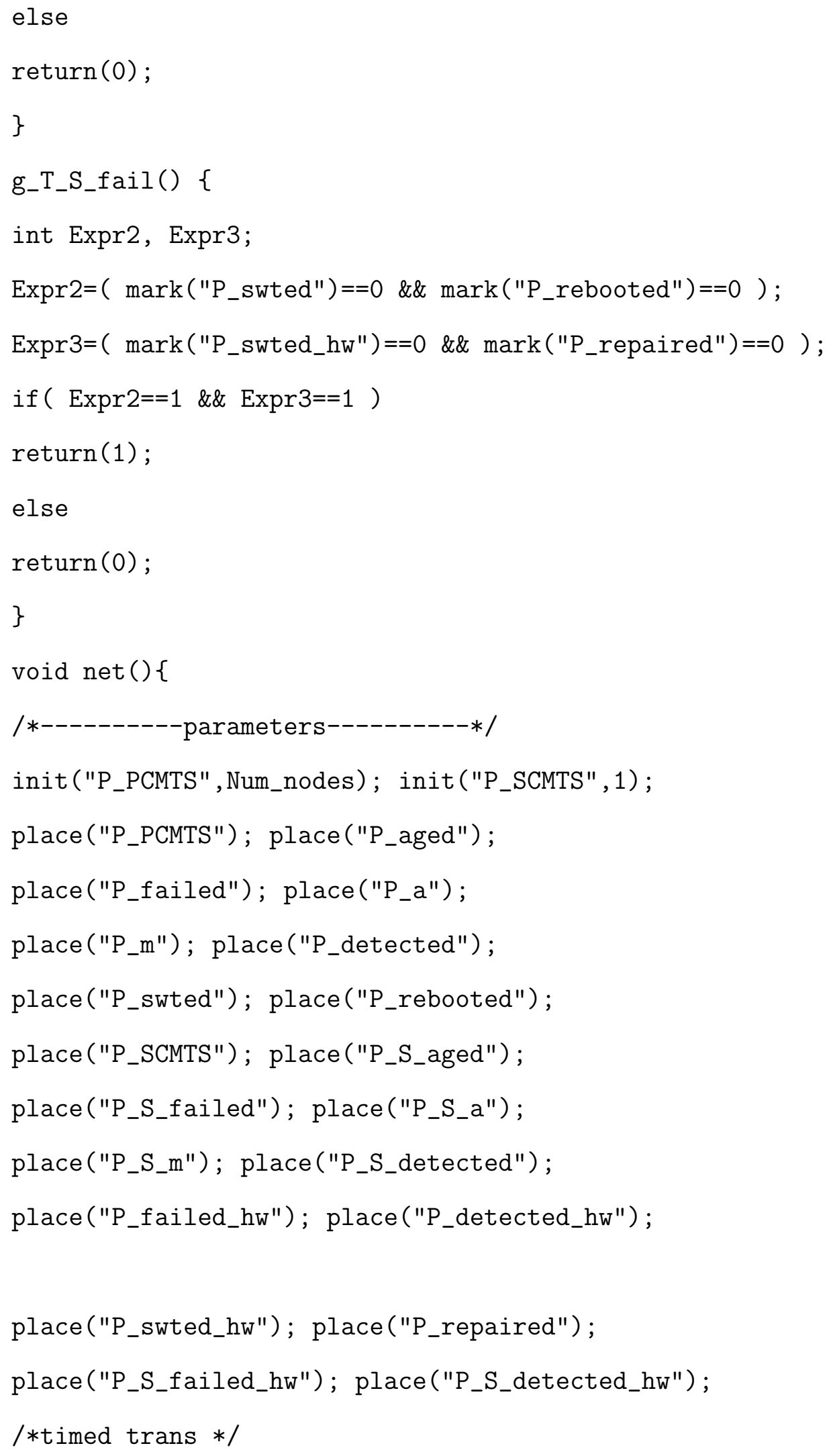




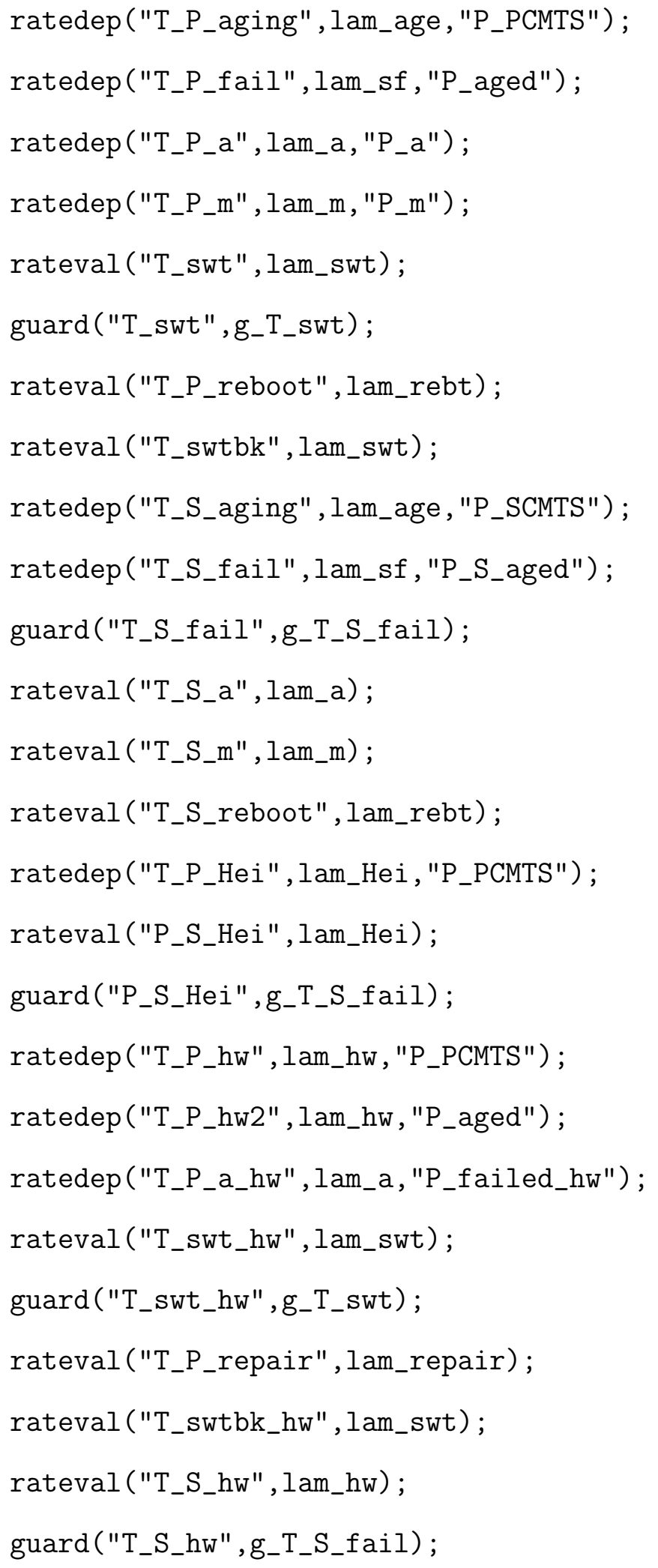




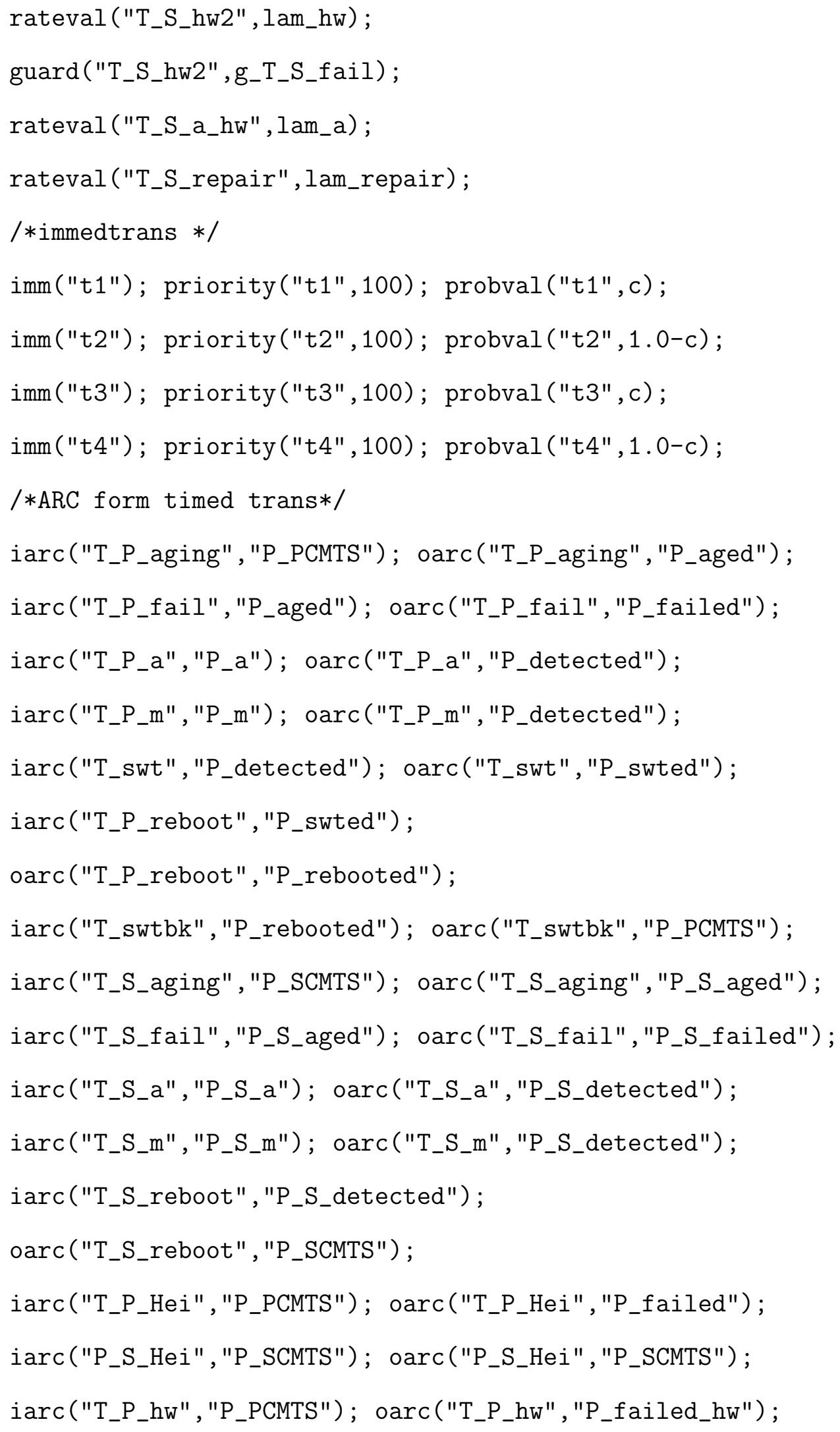




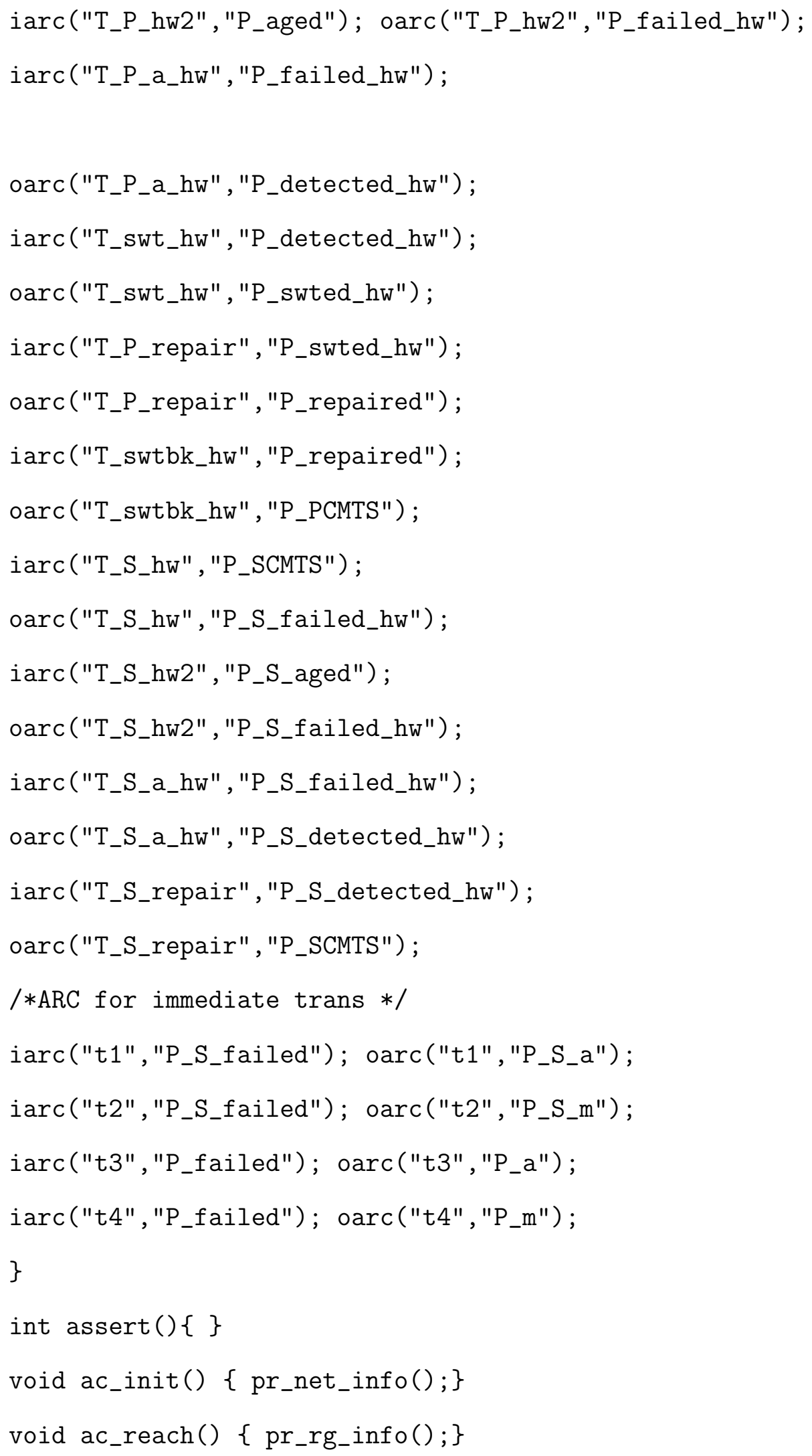




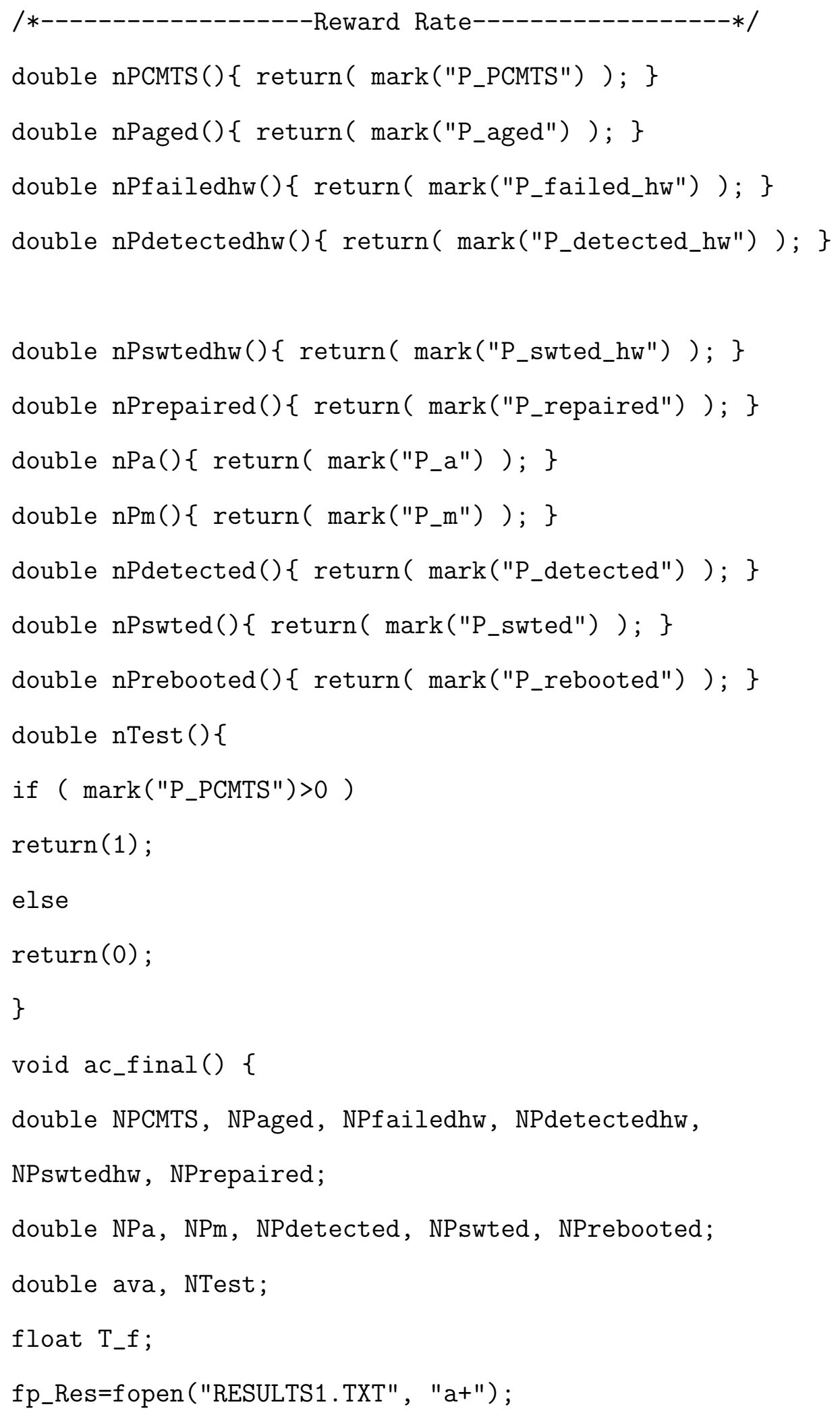




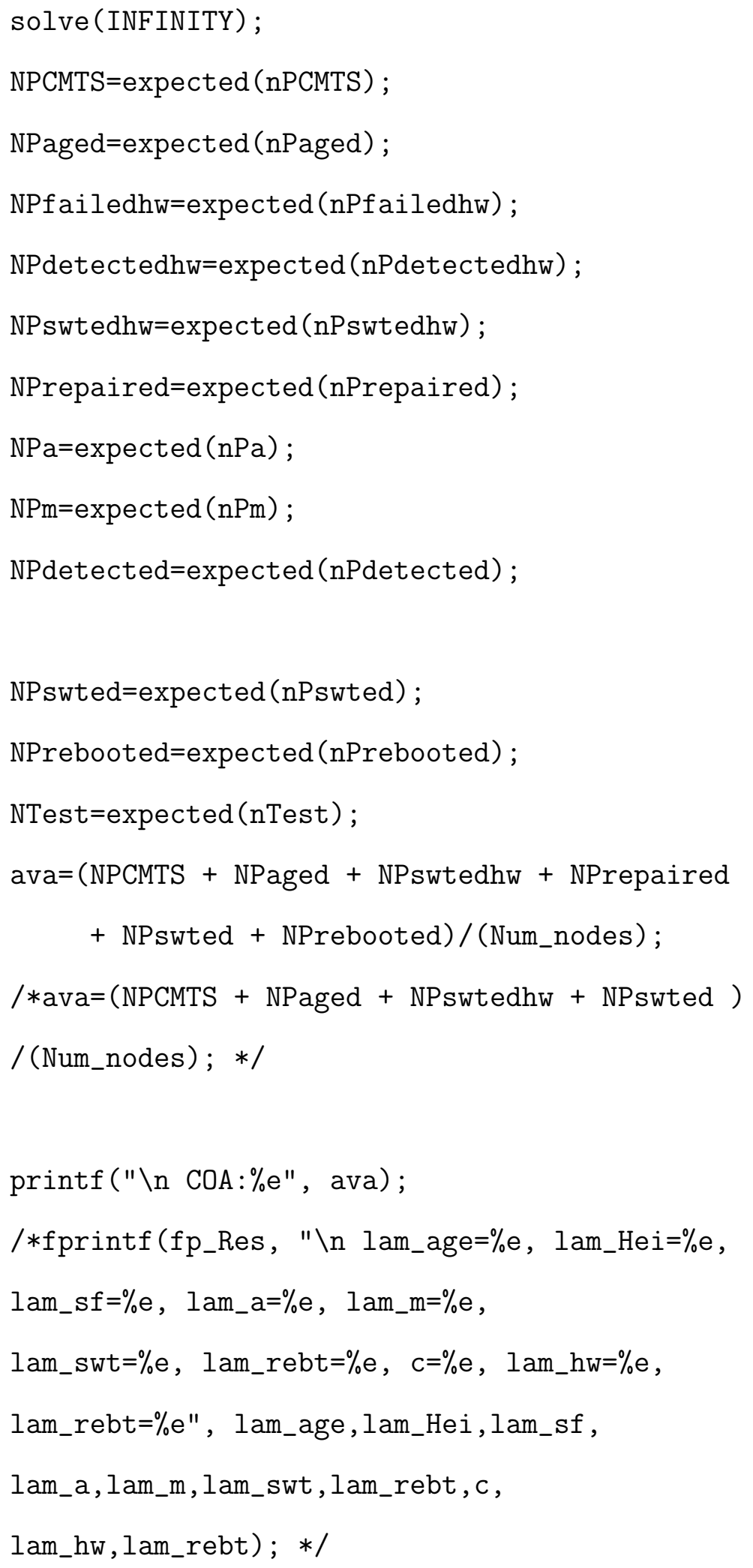




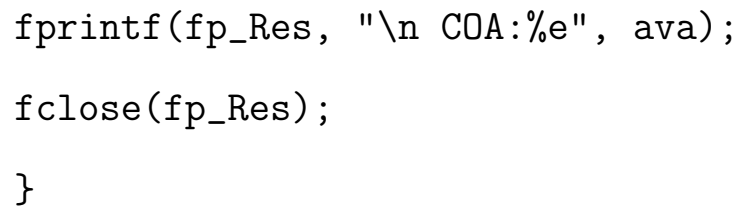

A.5 Mathematica Input File for Section 7

ClearAll [Evaluate[Context [] $<>~ " * "]]$;

SetDirectory ["C: \\My_Mathematica_Work"] ;

maxSampleSize $=100000 ;$

calculateNumSamplesLowerFailure [pointEstimate_, upperLimit_, confLevel_] :=

Module $[\{z, x 1, x 2, x, r$, resultApp, resultExact $\}$, $z=$ Quantile[NormalDistribution $[0,1],($ confLevel)];

$\mathrm{x}=$ pointEstimate/upperLimit;

$\mathrm{x} 1=(\mathrm{x} *(-1+\mathrm{z} * \mathrm{z})+\mathrm{x} * \mathrm{x} *(1+\mathrm{z} * \mathrm{z})-$ $2 * \operatorname{Sqrt}[(x * x * z * z *(-1+x+x * z * z))]) /(4 *(1-2 * x+x * x)) ;$

$\mathrm{x} 2=(\mathrm{x} *(-1+\mathrm{z} * \mathrm{z})+\mathrm{x} * \mathrm{x} *(1+\mathrm{z} * \mathrm{z})+$ $2 * \operatorname{Sqrt}[(\mathrm{x} * \mathrm{x} * \mathrm{z} * \mathrm{z} *(-1+\mathrm{x}+\mathrm{x} * \mathrm{z} * \mathrm{z}))]) /(4 *(1-2 * \mathrm{x}+\mathrm{x} * \mathrm{x}))$;

resultApp $=$ Floor $[\operatorname{Max}[\mathrm{x} 1, \mathrm{x} 2]]$;

resultExact $=$

r /. FindRoot [

InverseCDF [ChiSquareDistribution[2 $r$ ], confLevel] $* x / 2==r,\{r$, resultApp/2, resultApp*2\}, Method $\rightarrow$ Brent];

resultExact $=$ Floor $[$ resultExact $] ;(*$ To Do: If $[$ resultExact $<$ 1] ; *$,

If [resultExact > maxSampleSize, resultExact $=$ maxSampleSize,]; 


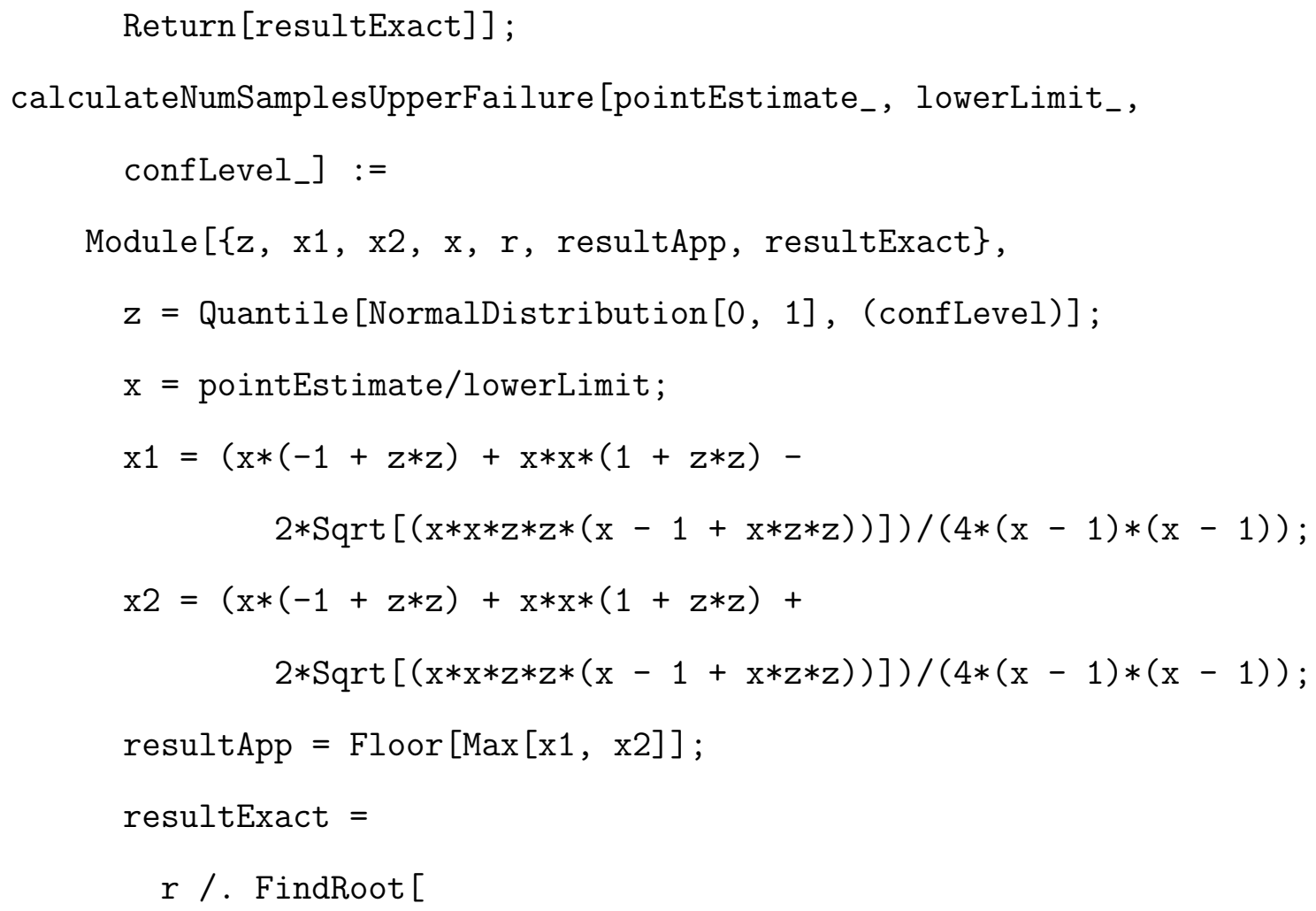




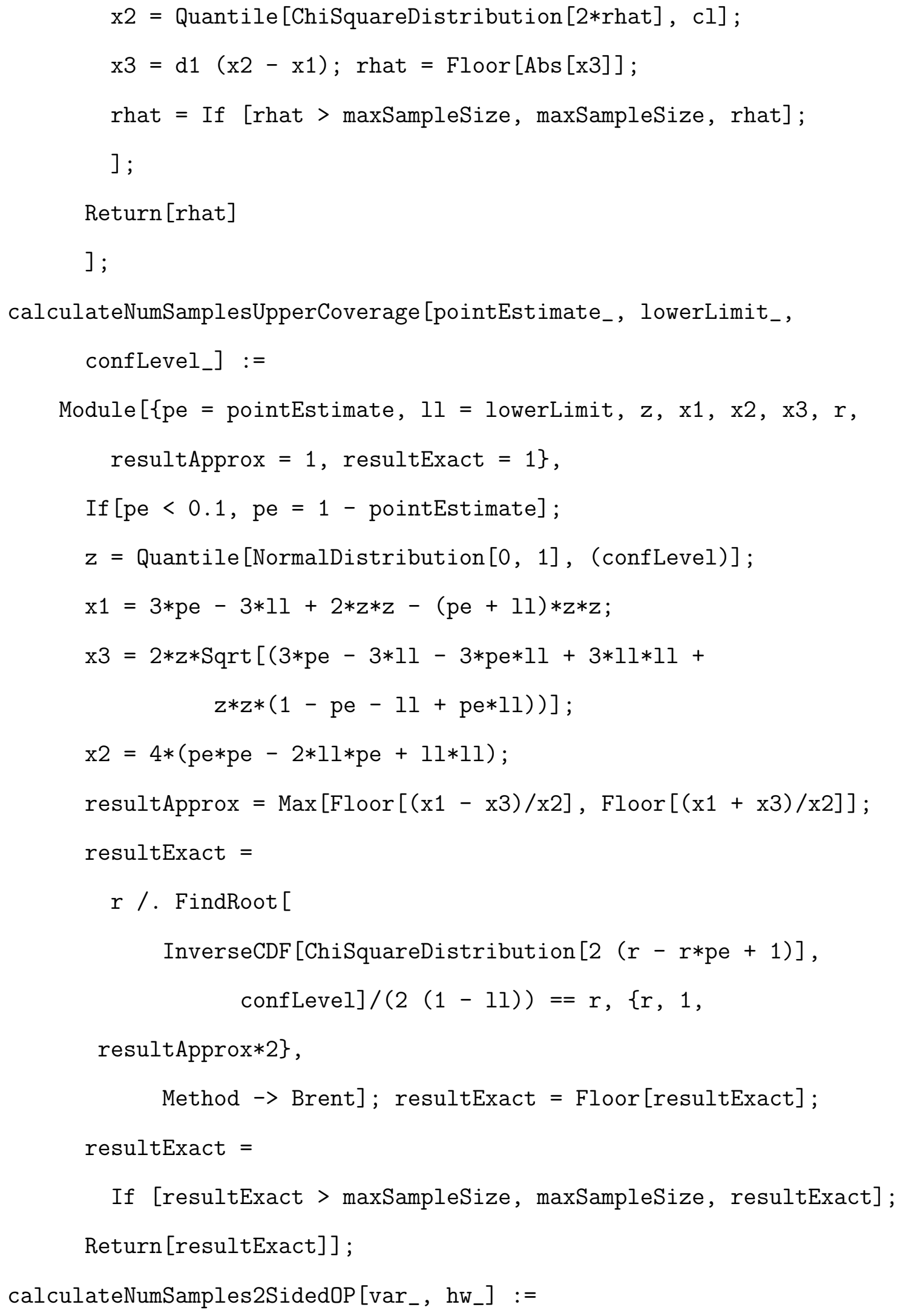




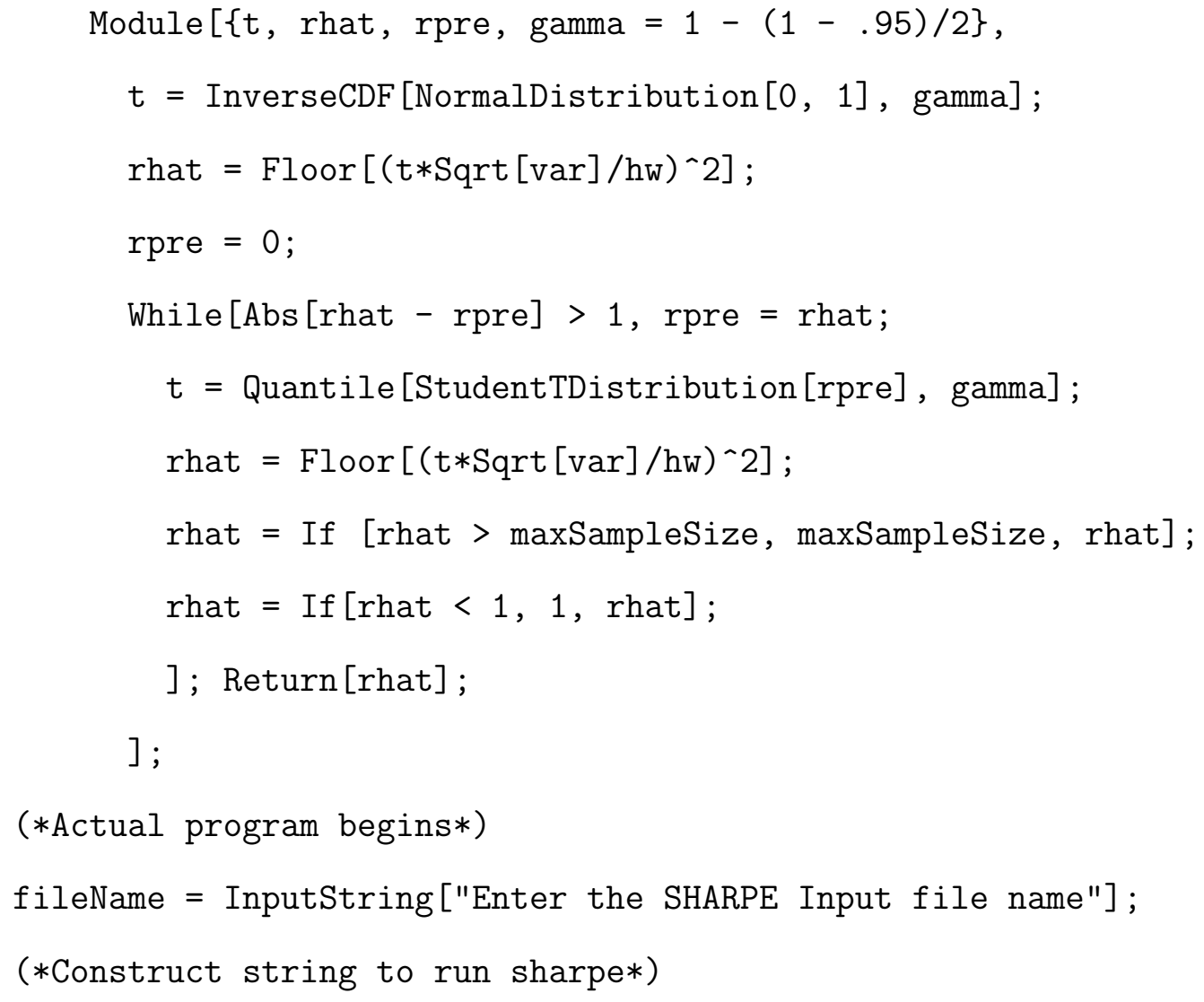

(*Actual program begins*)

fileName = InputString["Enter the SHARPE Input file name"]; (*Construct string to run sharpe*)

sharpeFileNamestring $=$

"sharpe " <> fileName <> " >> finalOutputCorrelated.out";

(*Remove the outputfile if it exists*)

DeleteFile["finalOutputCorrelated.out"] ;

(*Create an empty parametervalues file if it doesnot exist*)

s = OpenWrite["parametervalues"];

Close $[\mathrm{s}]$;

(*Record the point estimate of the measures*)

Run [sharpeFileNamestring] ;

(* To get all the input parameter names, include the all the \ parameters in a separate file using the syntax: bind parameterName \ parameterValue. 
This file should be the first included SHARPE file*)

(* From SHARPE code, get the name of the file included, which has the \ param names and values.*)

$$
\mathrm{x}=\text { FindList }[\text { fileName, "include", 1]; }
$$

$\mathrm{x} 1=$ StringSplit $[\mathrm{x}]$;

paramFileName $=x 1[[1,2]]$;

(*Print ["ParamFileName is ", paramFileName];*)

(* Now open the file of name just found and read each parameter name \ and values.

Store the \# of params in numParameters and Parameter Values in $\backslash$ array paramValues*)

paramNames $=$

ReadList [paramFileName, Record, RecordSeparators $\rightarrow\{\{$ "bind " $\},\{"$ " $\}\}]$;

numParameters = Length $[$ paramNames $]$;

(*Print ["Num Params is ", numParameters]; *)

paramValues = Array $[$, numParameters $]$;

paramSampleSize = Array [, numParameters $]$;

sNum = OpenRead $[$ paramFileName $]$;

For $[i=1, i<$ numParameters $+1, i++$,

Skip [sNum, \{Word, Word\}];

paramValues $[[i]]=\operatorname{Read}[\mathrm{sNum}$, Number $]$;

(*Print ["Param Values is ", paramValues[[i]]];*)

];

Close [sNum] ;

(*get the output parameter names *) 


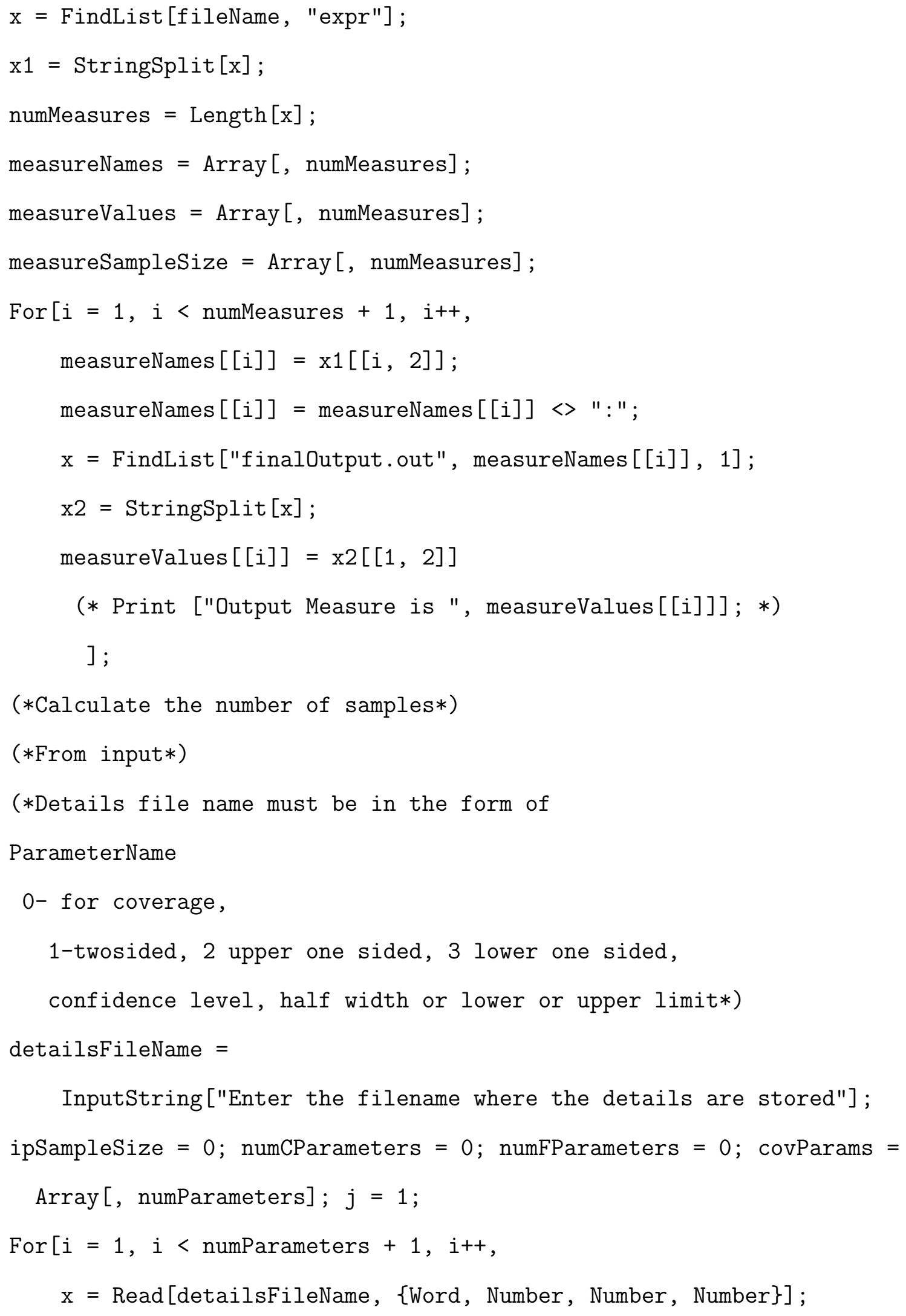




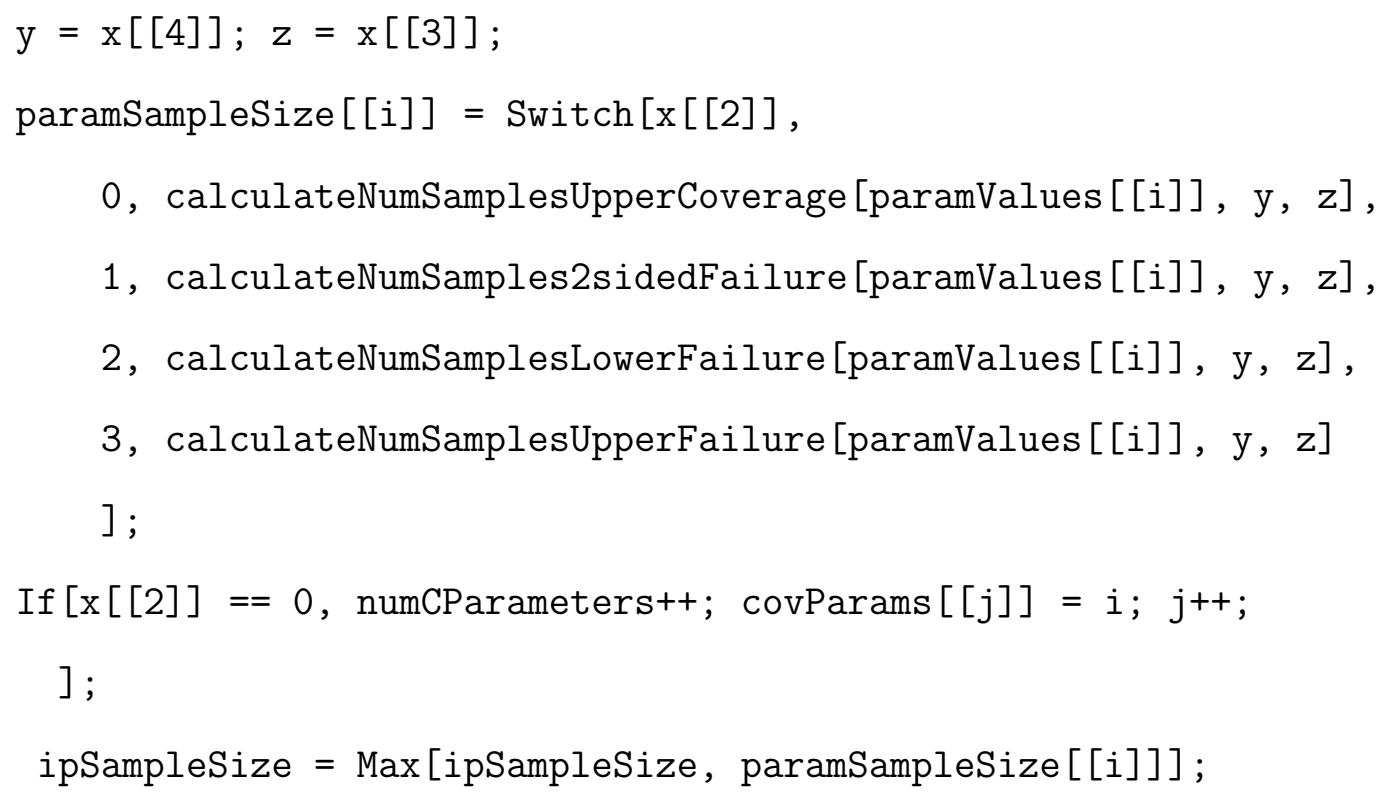


tempQuant $=$

RandomReal [UniformDistribution [\{lowerUni, upperUni\}]] ;

parameterValue $=0$;

For $[j=1, j<$ numParameters $+1, j++$,

If $[$ paramType $[[j]]==1$,

parameterValue $=$

Quantile[GammaDistribution [paramSampleSize [[j]], paramValues $[[j]] /$ paramSampleSize $[[j]]]$, tempQuant] ; (* Print ["Param is a rate param and values is ", paramNames $[[j]]$, paramValues $[[j]]] ; *)$

paramArray2D $[[j]][[\mathrm{m}]]=$ parameterValue;

$\mathrm{k}=\mathrm{Floor}[\operatorname{paramSampleSize}[[j]] * \operatorname{paramValues}[[j]]]$;

parameterValue $=$

Quantile[

BetaDistribution $[k+1$, paramSampleSize $[[j]]-k+1]$, tempQuant];

paramArray $2 \mathrm{D}[[\mathrm{j}]][[\mathrm{m}]]=$ parameterValue;

(* Print ["Param is a coverage param and values is ", paramNames $[[j]]$, paramValues $[[j]]] ; *)$

] ;

];

];

(* Print [" The paramArray2d ", paramArray2D]; *)

For $[i=1, i<$ numParameters $+1, i++$, tempArray $=$ paramArray2D $[[i]]$;

tempArrayRand = RandomSample [tempArray]; 


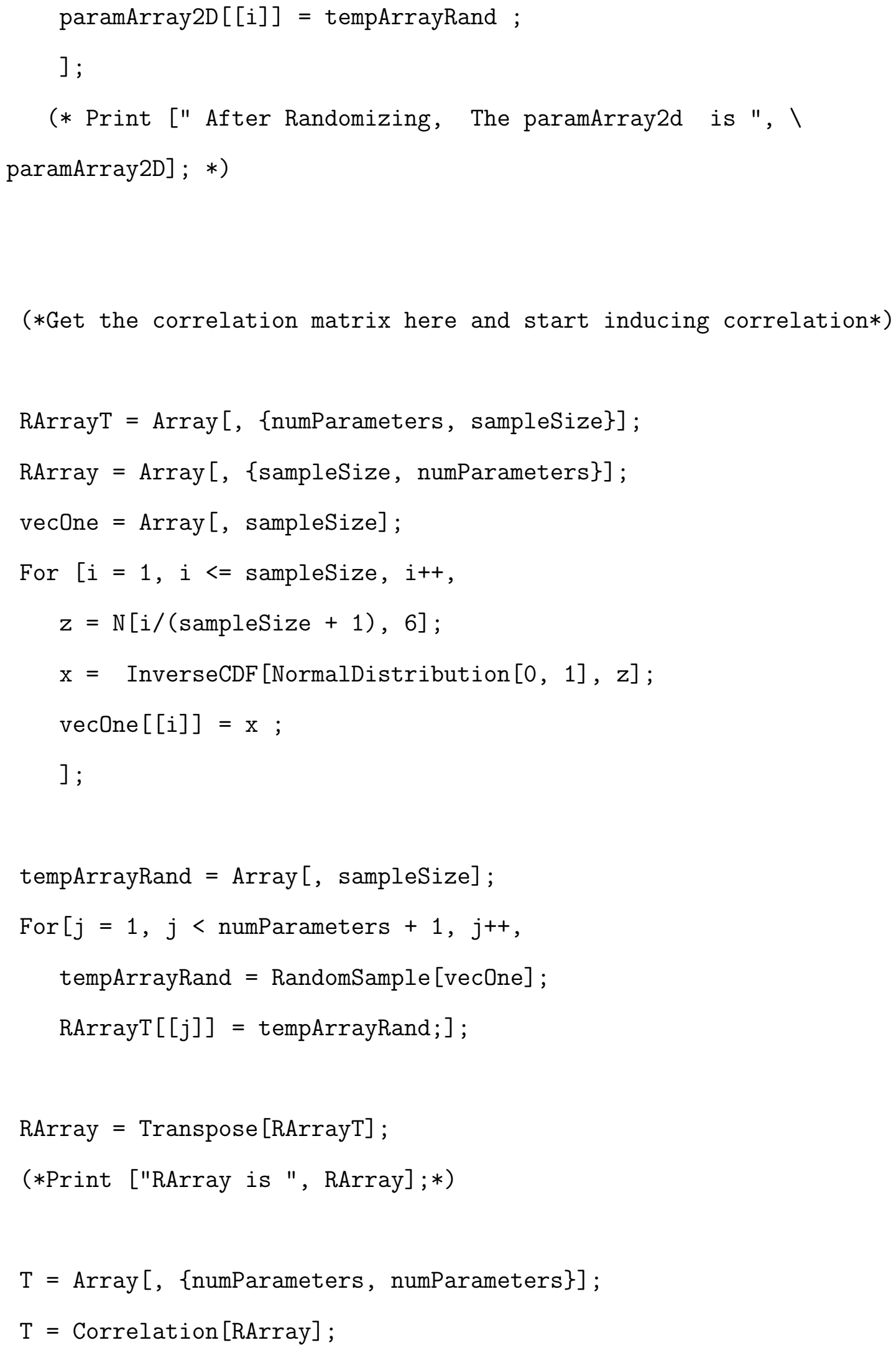




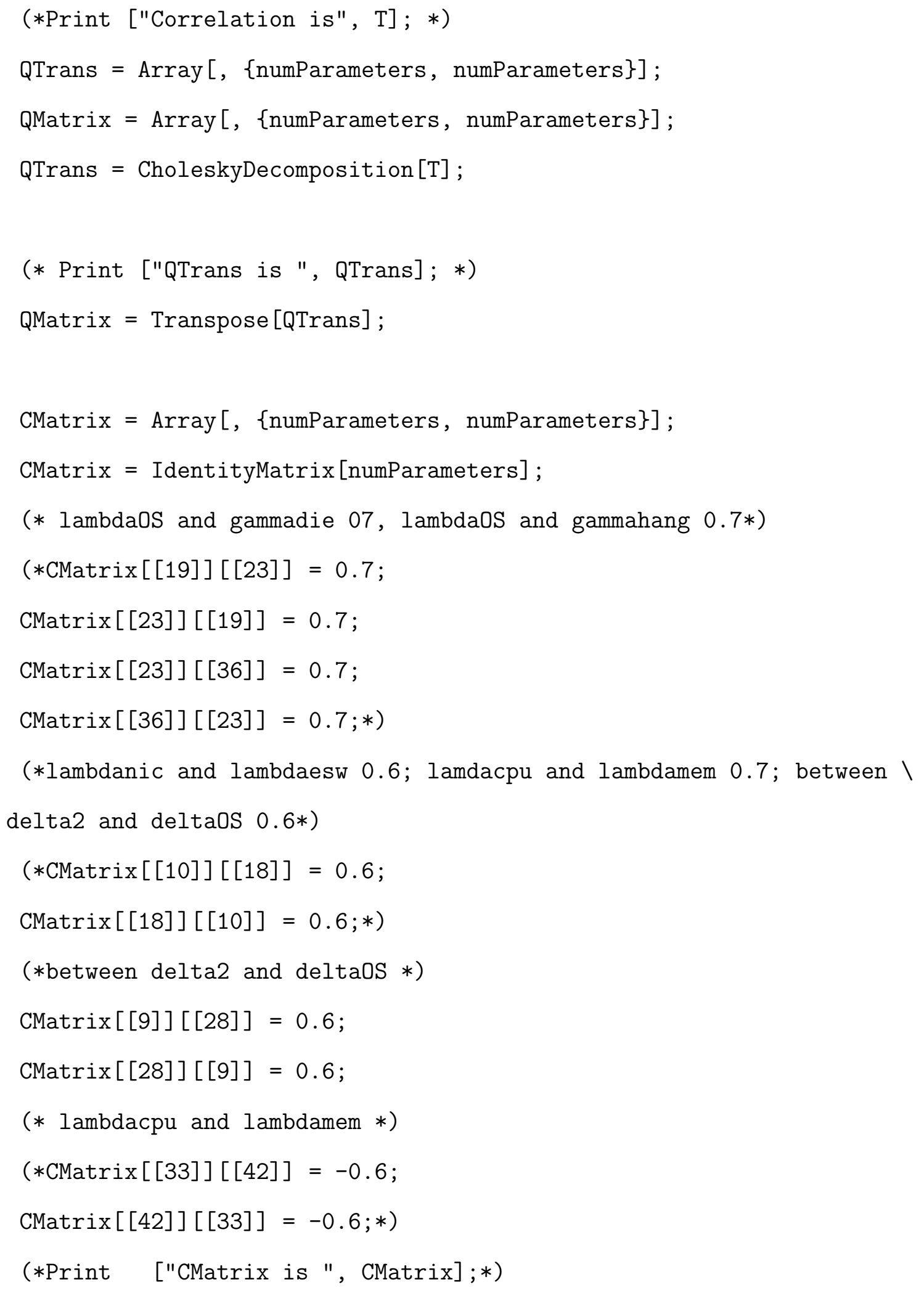




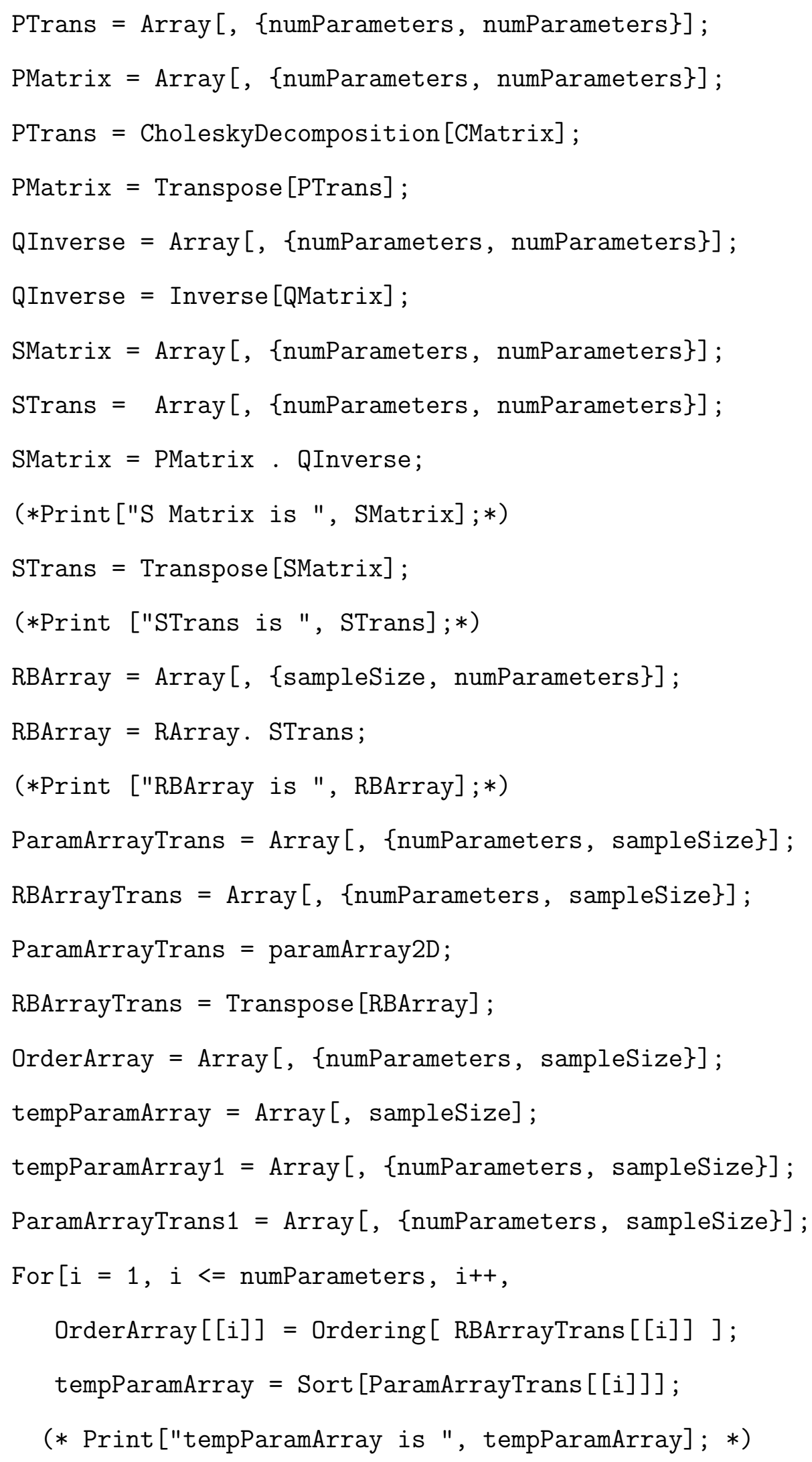




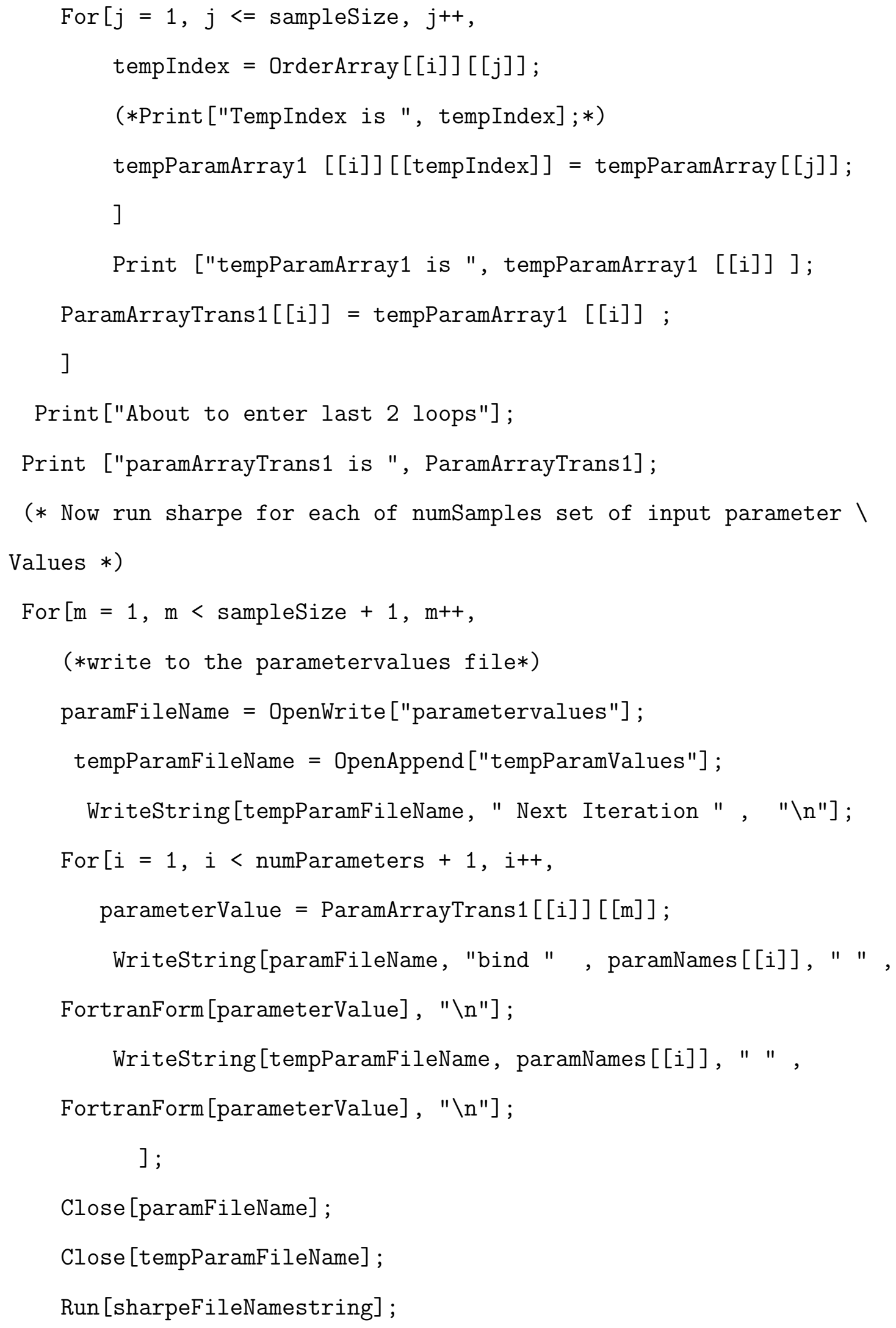


Print ["mth execution of sharpe completed ", m]; ];

]

A.6 Mathematica Input File for Section 8.1.1

(* The part determining number of samples implemented by Amita Devaraj*)

ClearAll [Evaluate[Context [] $<" * *$ ] ;

SetDirectory [

"C: \\My_Mathematica_Work"] ;

maxSampleSize $=100000$;

(*Functions*)

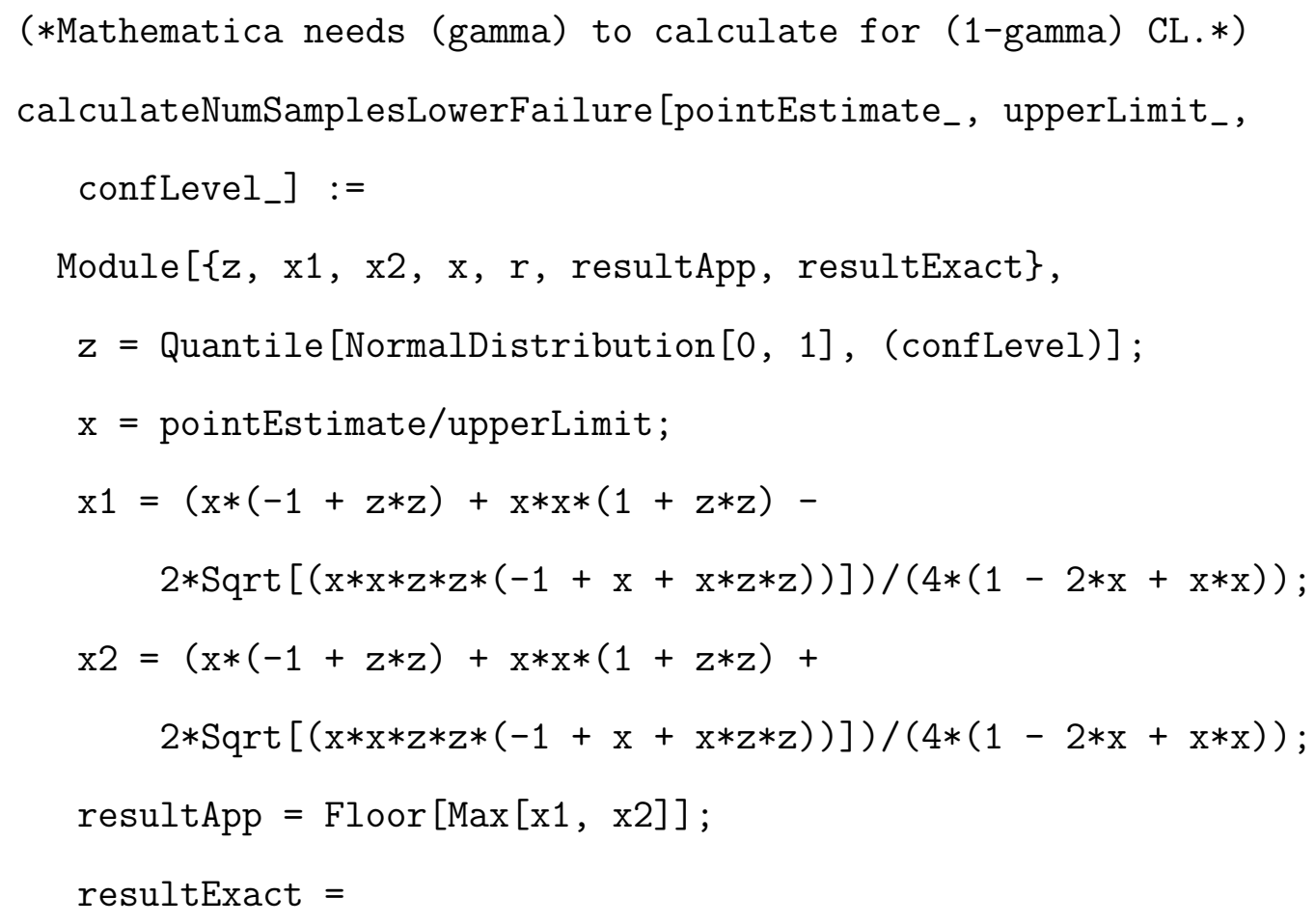




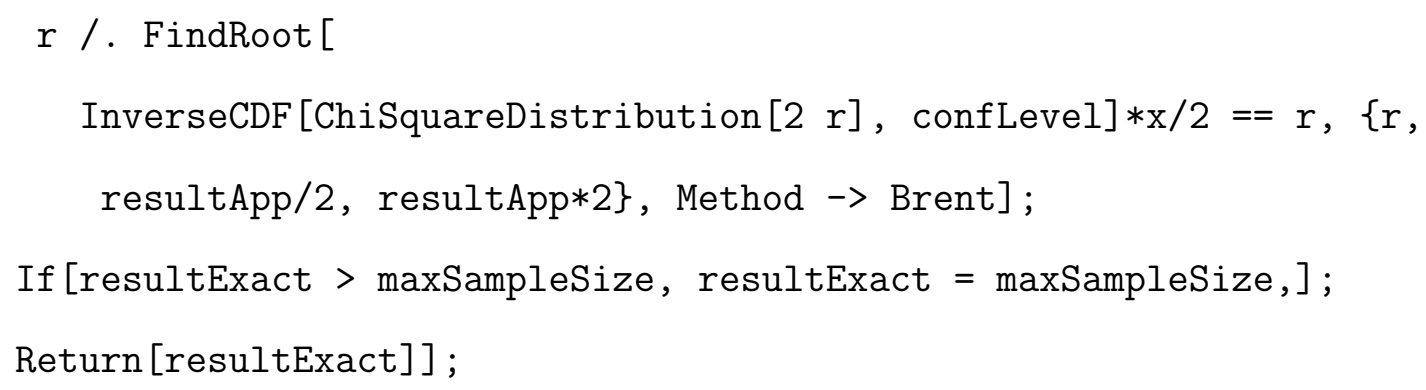




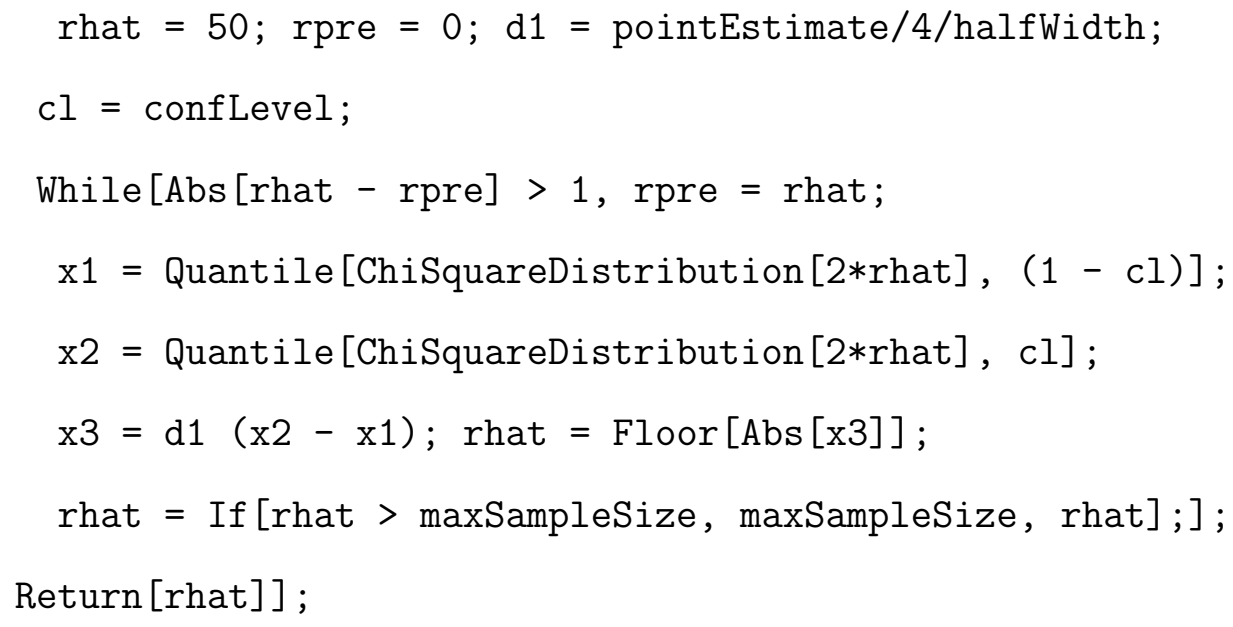




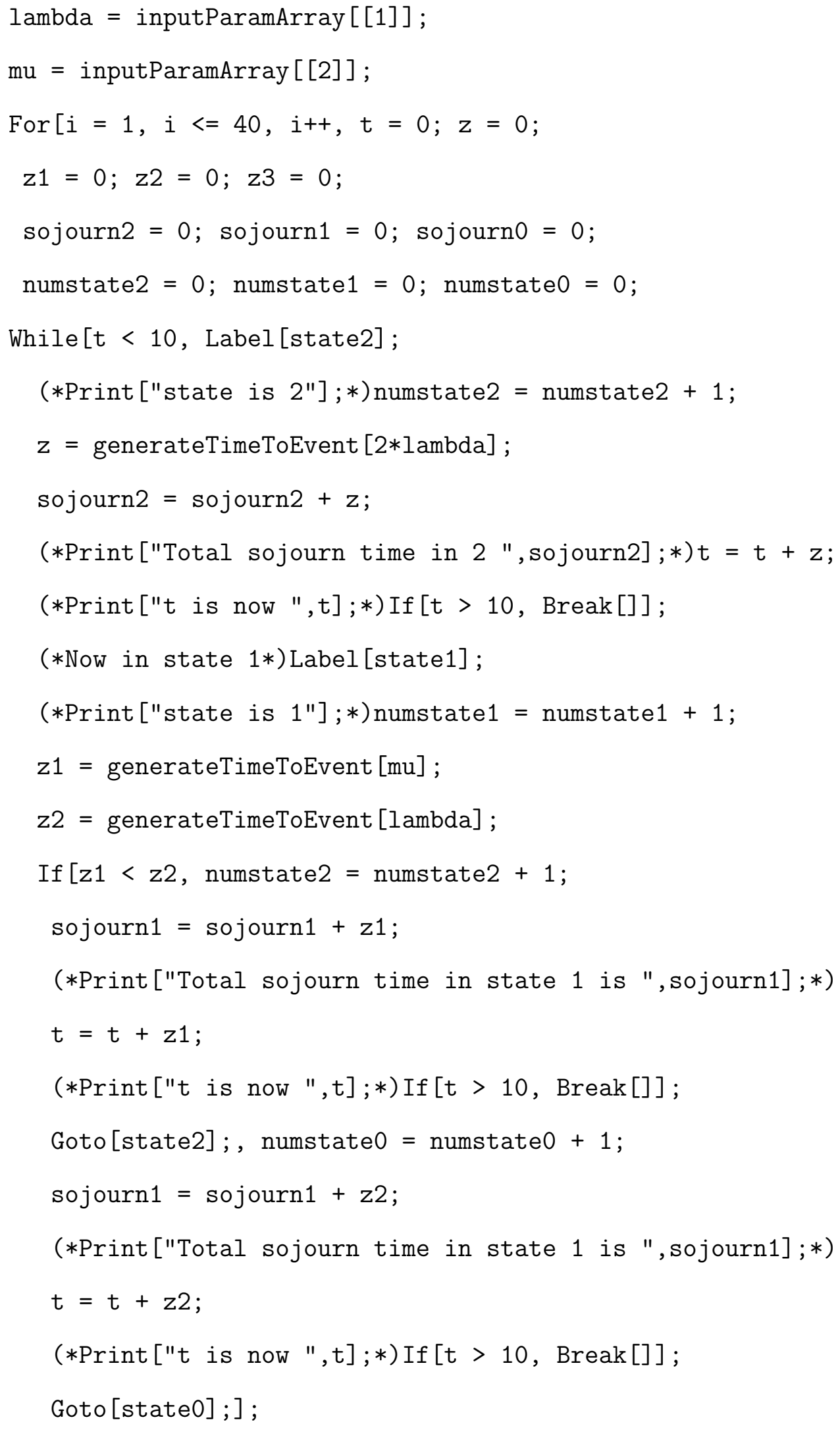




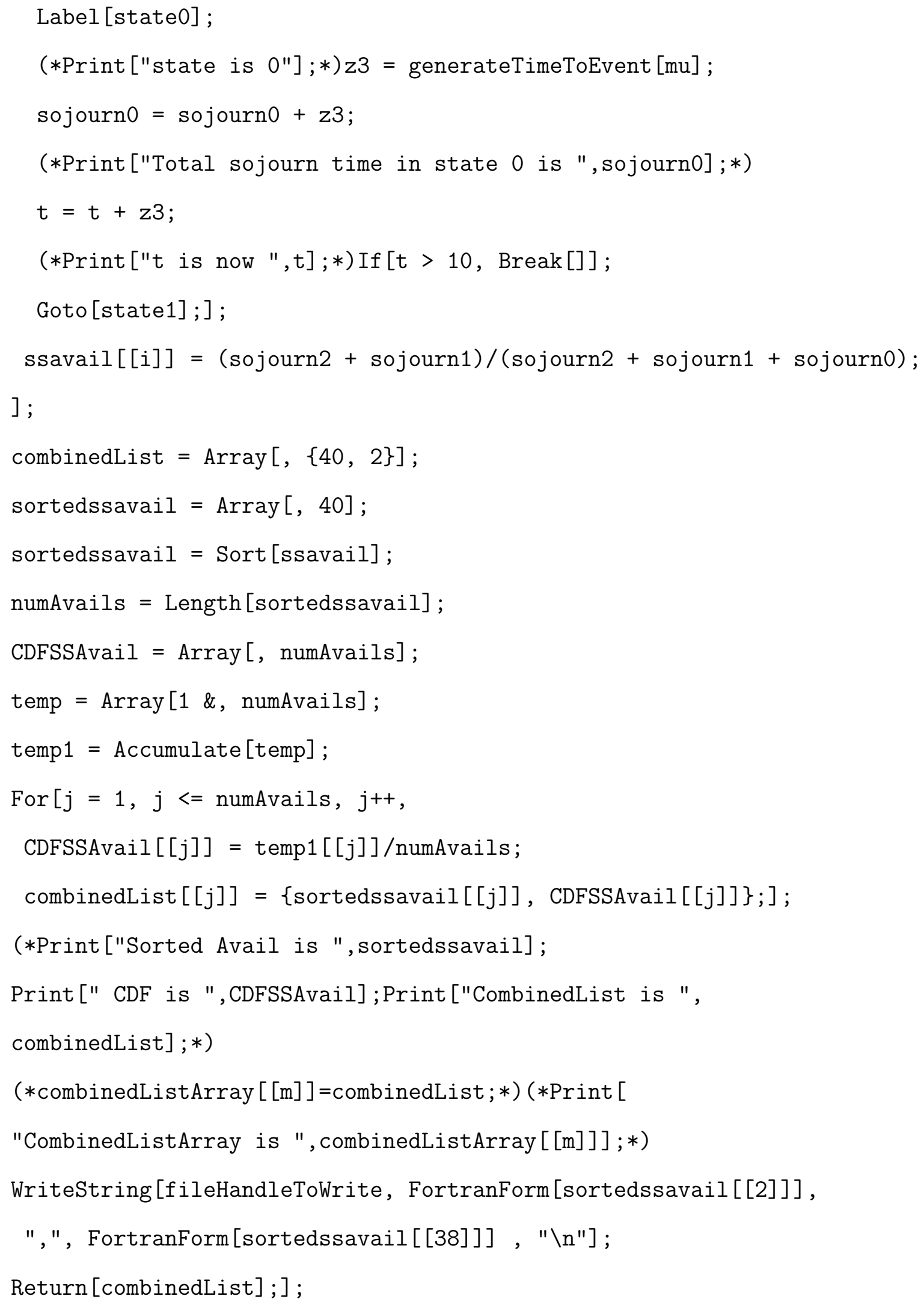




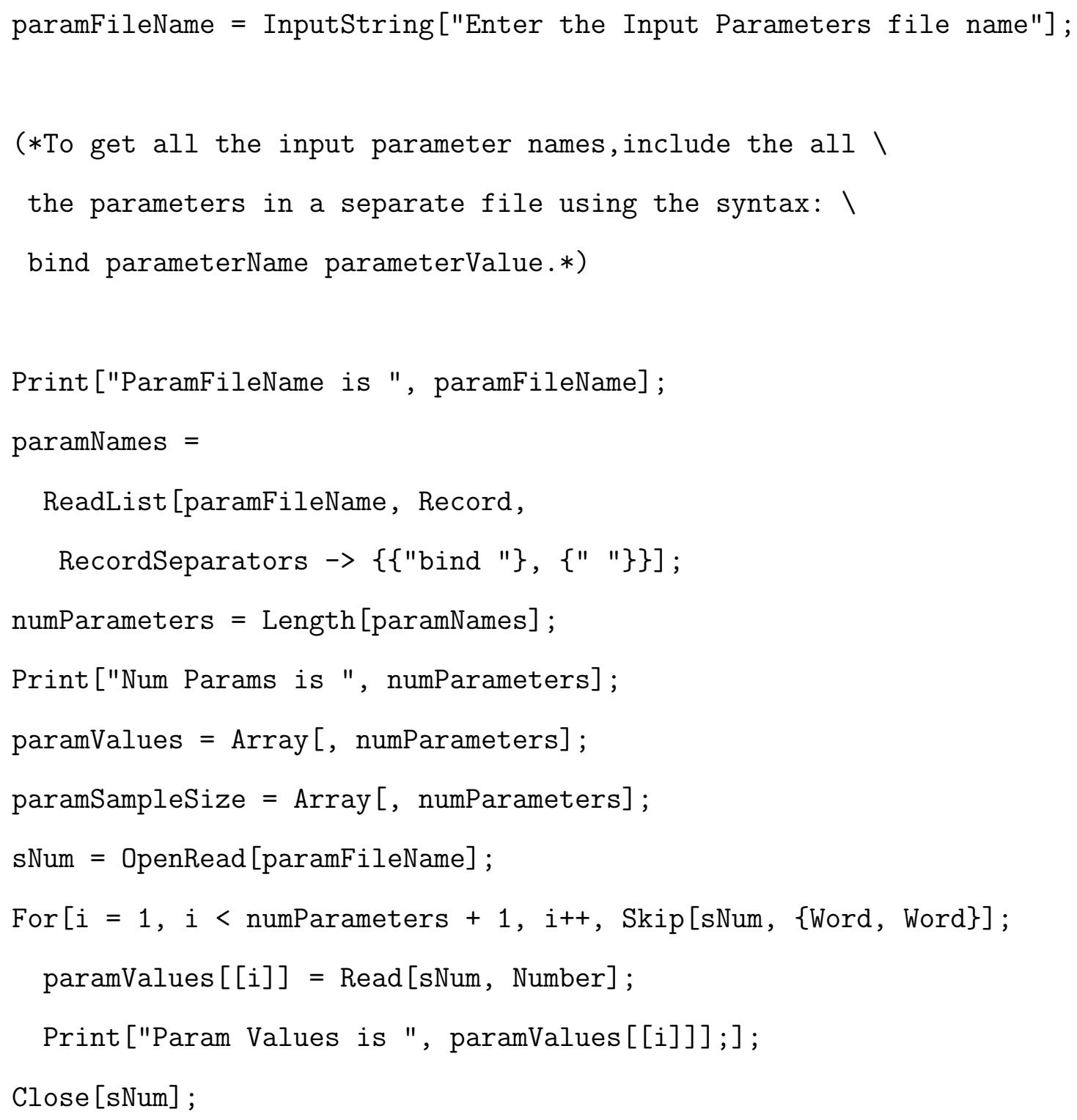




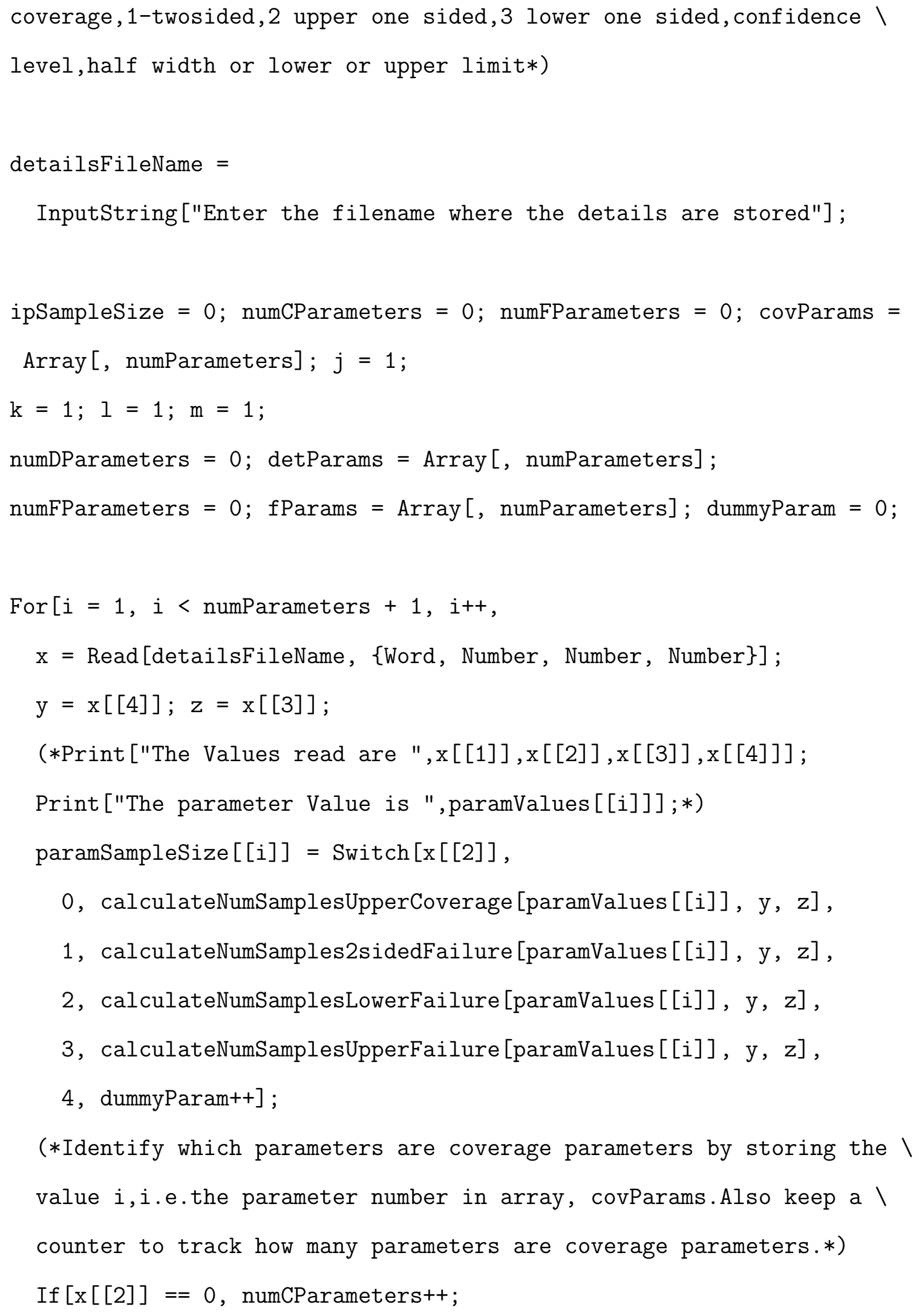




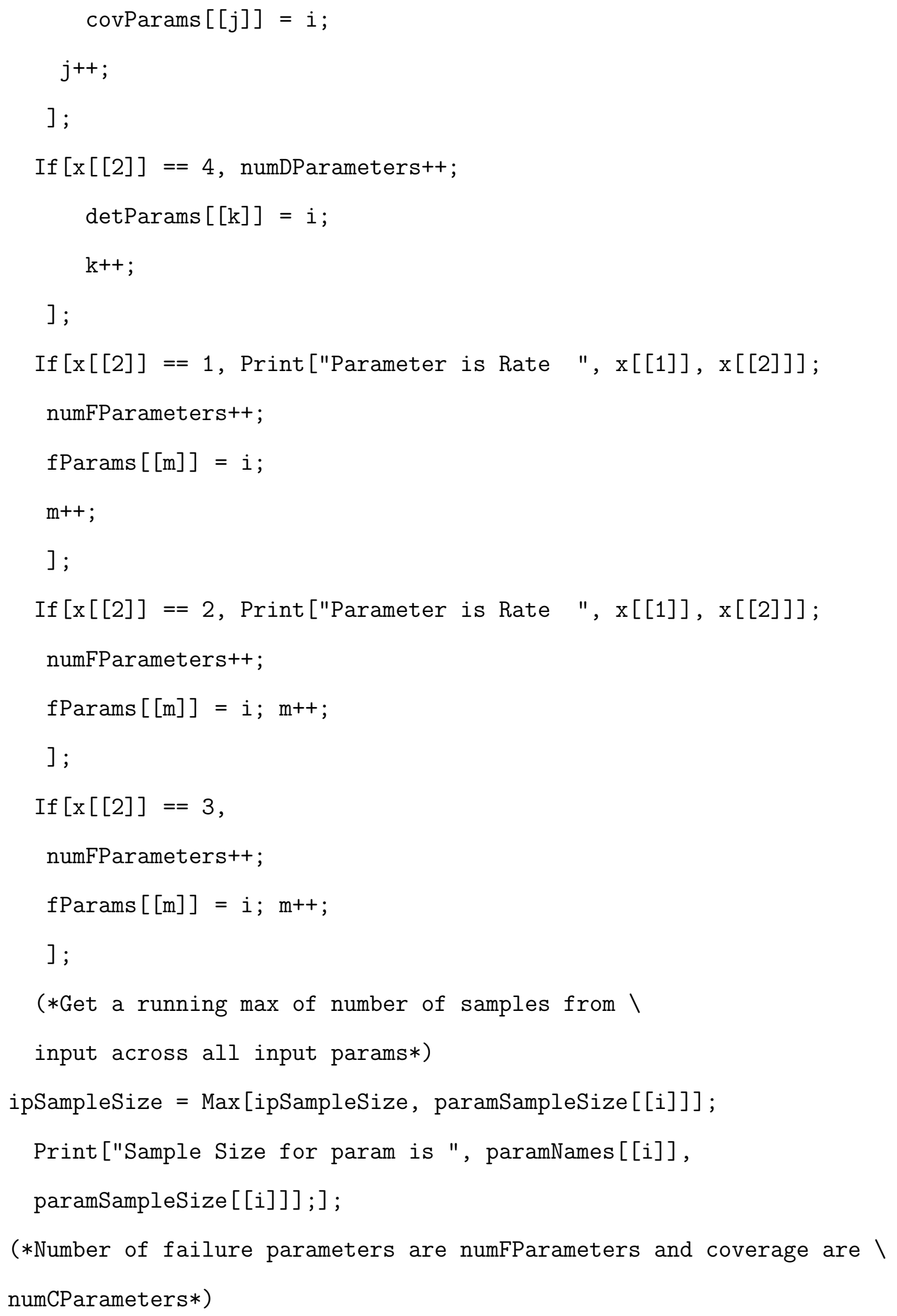




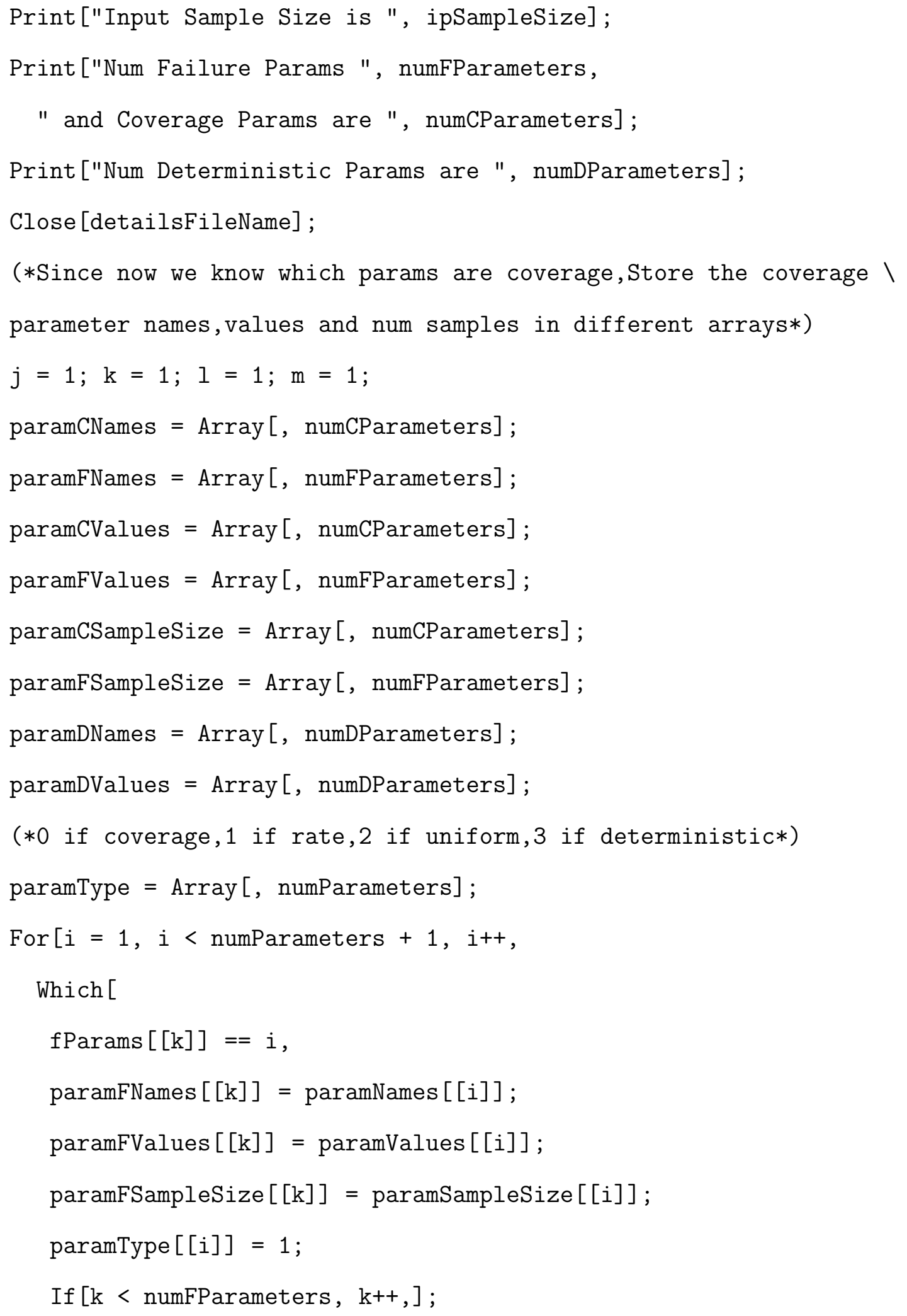




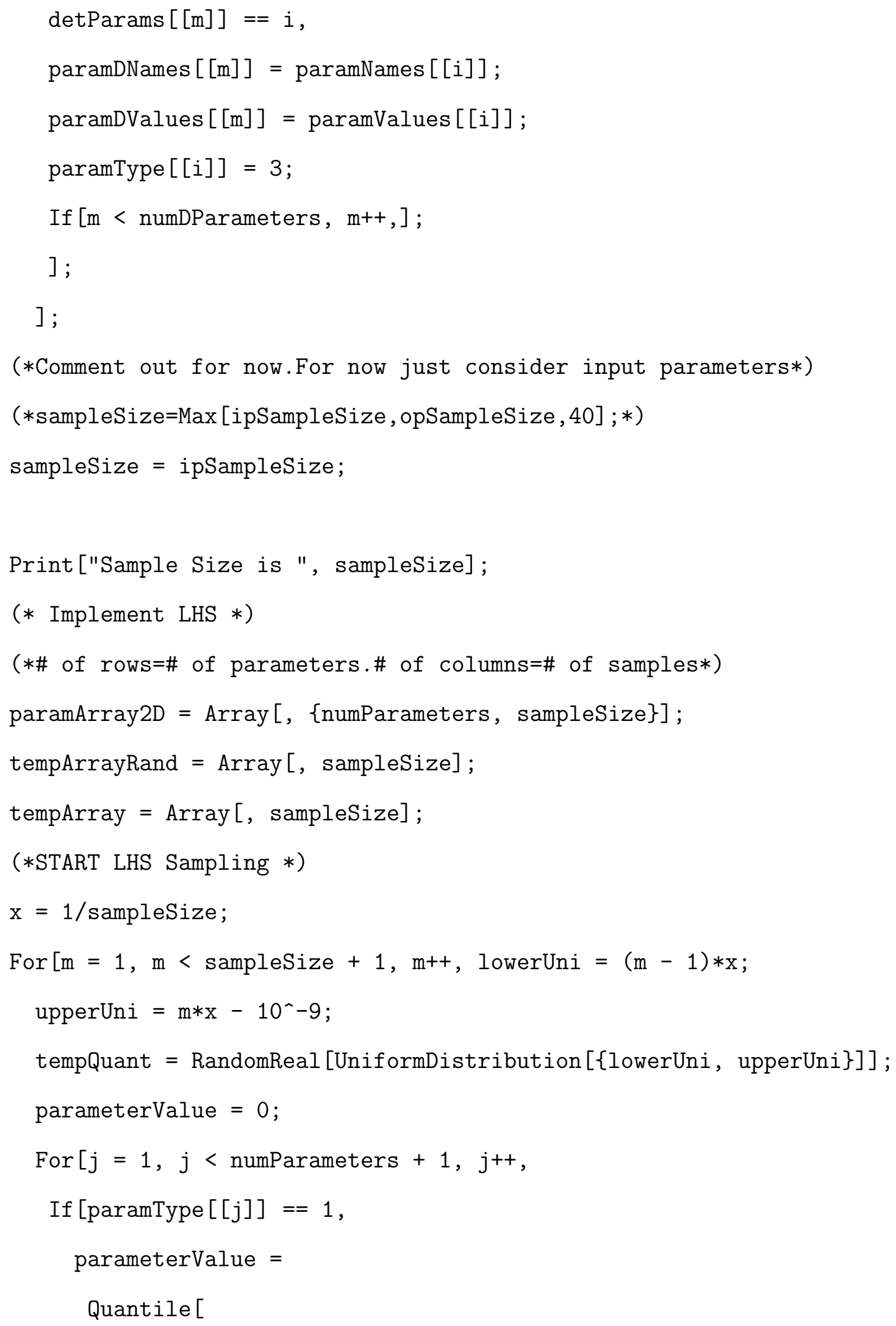




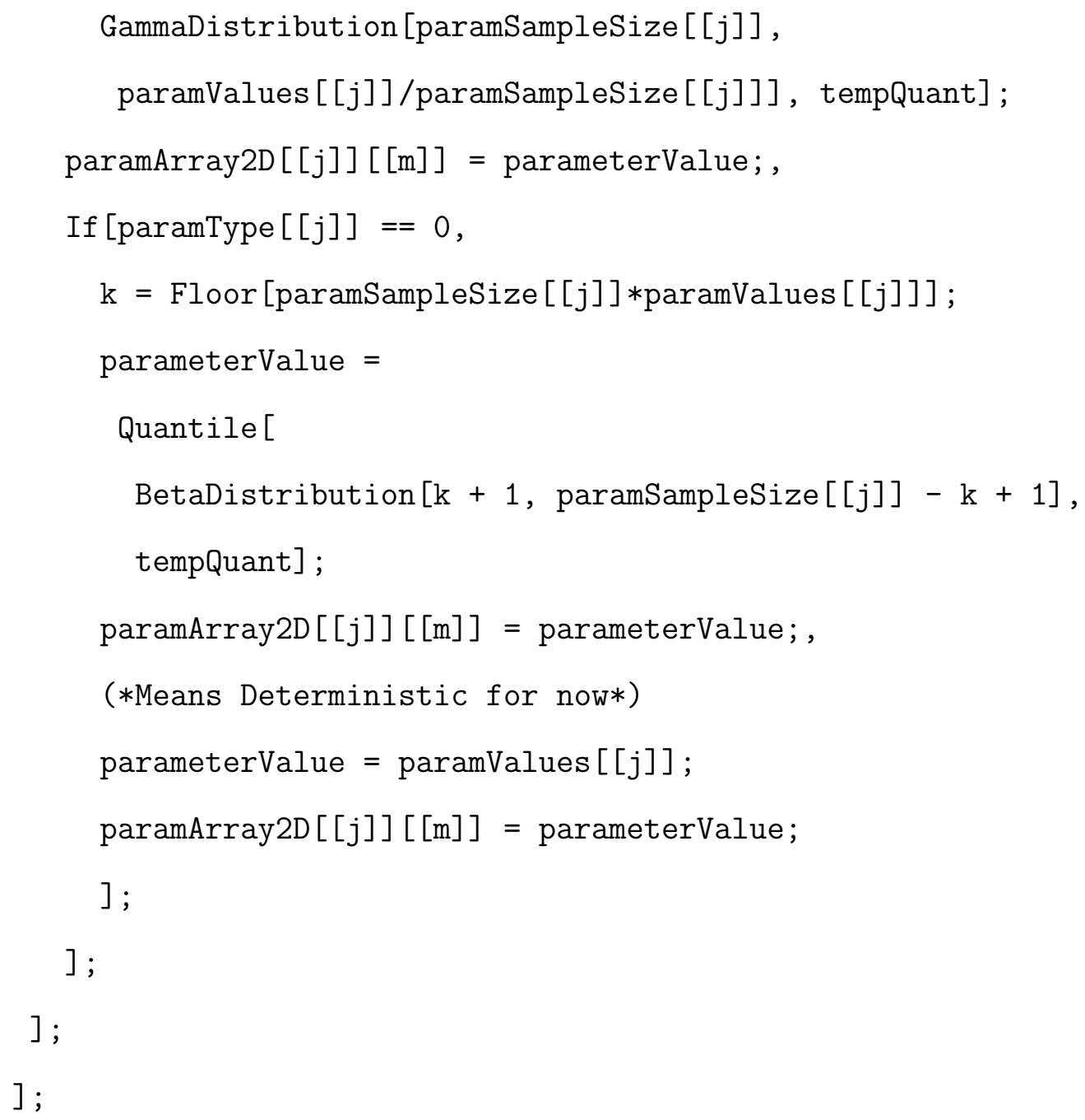




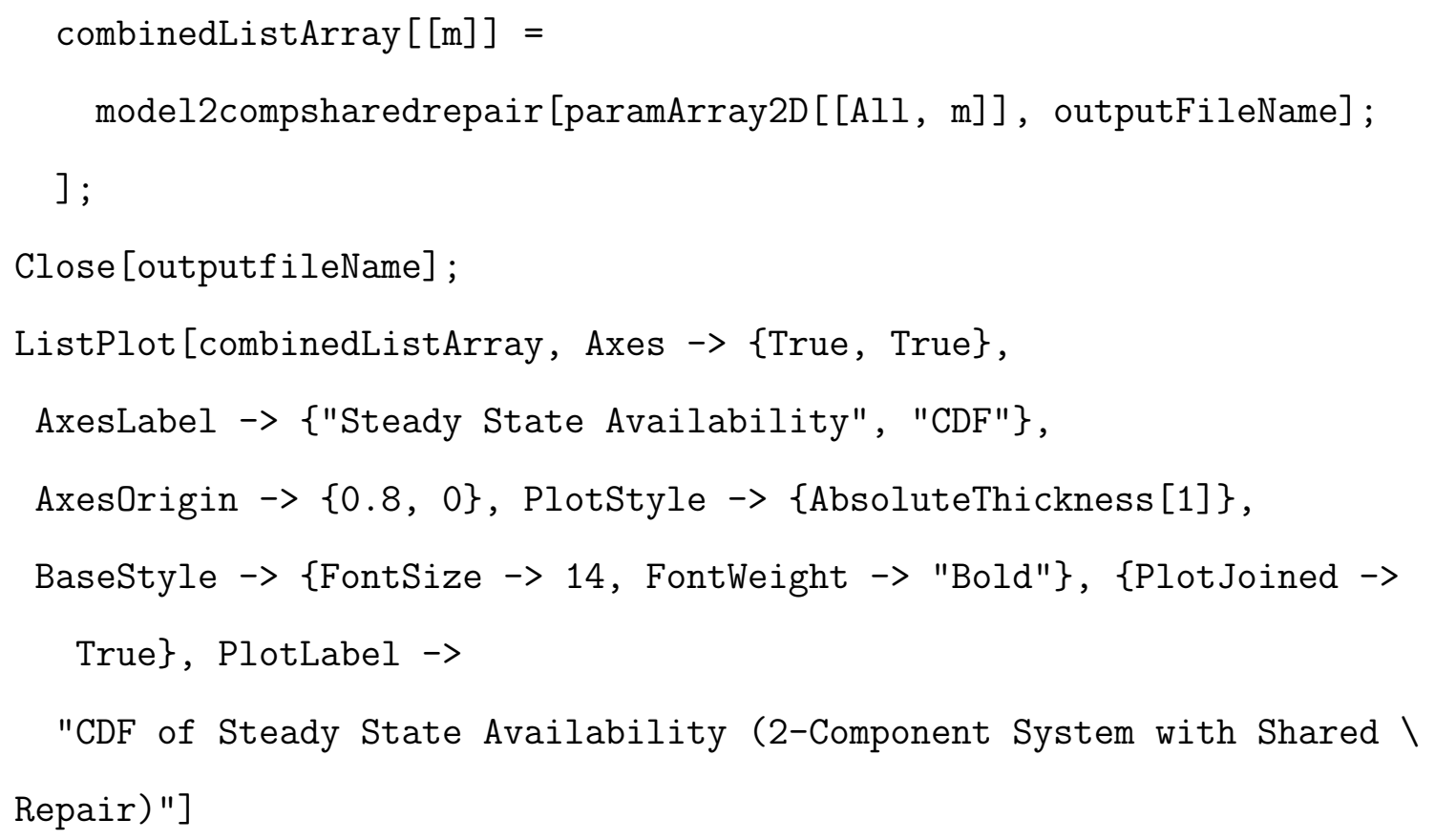

A.7 Mathematica Input File for Section 8.2

ClearAll [Evaluate[Context [] $<>" * "]]$;

SetDirectory [

"C: \\My_Mathematica_Work"] ;

maxSampleSize $=100000$;

(*Functions*)

(*Mathematica needs (gamma) to calculate for (1-gamma) CL.*)

calculateNumSamplesLowerFailure[pointEstimate_, upperLimit_, 


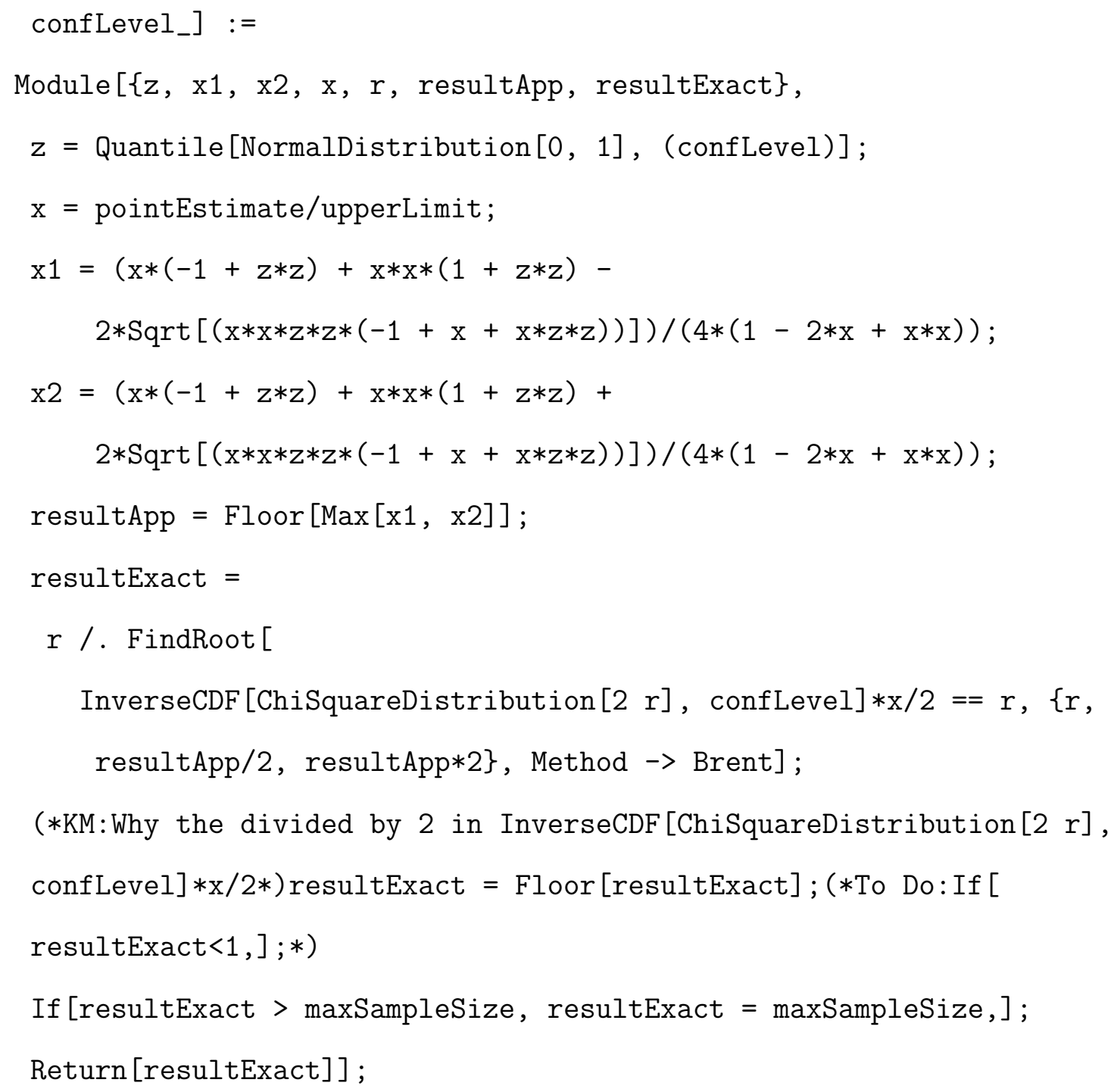

calculateNumSamplesUpperFailure [pointEstimate _, lowerLimit_, confLevel_] :=

Module $[\{z, x 1, x 2, x, r$, resultApp, resultExact $\}$, $z=$ Quantile[NormalDistribution $[0,1],($ confLevel)]; 
$2 * \operatorname{Sqrt}[(\mathrm{x} * \mathrm{x} * \mathrm{z} * \mathrm{z} *(\mathrm{x}-1+\mathrm{x} * \mathrm{z} * \mathrm{z}))]) /(4 *(\mathrm{x}-1) *(\mathrm{x}-1))$

resultApp $=$ Floor $[\operatorname{Max}[\mathrm{x} 1, \mathrm{x} 2]]$;

resultExact $=$

r /. FindRoot [

InverseCDF [ChiSquareDistribution[2 r], $(1-$ confLevel $)] * x / 2==$ $r,\{r$, resultApp/2, resultApp*2\}, Method $\rightarrow$ Brent] ;

resultExact $=$ Floor $[$ resultExact $] ;(*$ To Do:If $[$ resultExact $<1] ; *$, If [resultExact > maxSampleSize, resultExact $=$ maxSampleSize, ] Return [resultExact] ];

calculateNumSamples2sidedFailure [pointEstimate_, halfWidth_, confLevel_] :=

Module $[\{r h a t$, rpre, $d 1, \mathrm{cl}, \mathrm{x} 1, \mathrm{x} 2, \mathrm{x} 3, \mathrm{r} 1, \mathrm{r} 2$, ans1, ans2, result $\}$, rhat $=50 ;$ rpre $=0 ; d 1=$ pointEstimate $/ 4 /$ halfWidth;

cl = confLevel;

While [Abs [rhat - rpre] > 1, rpre = rhat; $\mathrm{x} 1=$ Quantile[ChiSquareDistribution [2*rhat], $(1-\mathrm{cl})]$; $\mathrm{x} 2=$ Quantile [ChiSquareDistribution [2*rhat], cl]; $\mathrm{x} 3=\mathrm{d} 1(\mathrm{x} 2-\mathrm{x} 1) ;$ rhat $=$ Floor $[\operatorname{Abs}[\mathrm{x} 3]]$; rhat $=$ If $[$ rhat $>$ maxSampleSize, maxSampleSize, rhat $] ;]$; $(*$ ans $1=r 1 /$. FindRoot [pointEstimate/2/r1*InverseCDF [ ChiSquareDistribution [2*r1] , (1-confLevel)/2]==(pointEstimatehalfWidth), \{r1, 1,2*rhat $\}$, Method->Brent] ; ans $2=r 2 /$. FindRoot [pointEstimate/2/r2*InverseCDF [ ChiSquareDistribution [2*r2], 1-(1-confLevel)/2] ==(pointEstimate+ halfWidth), \{r2, 1,2*rhat $\}$, Method->Brent] ; Print [ans1, ans2]; 
result $=\operatorname{If}[$ NumberQ[ans1]\&\&NumberQ [ans2], Floor [Max [ans1, ans2]], rhat];*) Return [rhat] ] ;

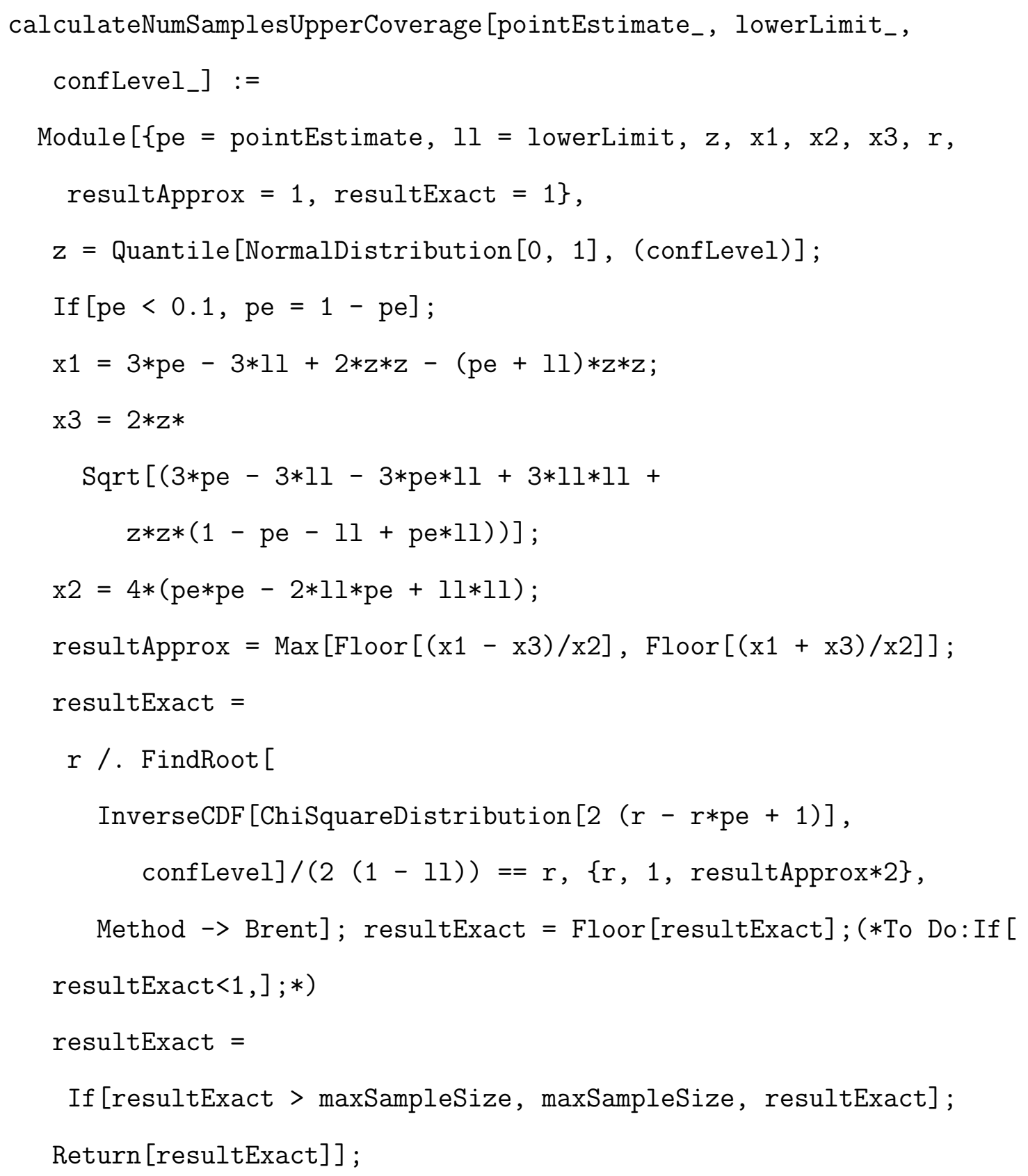




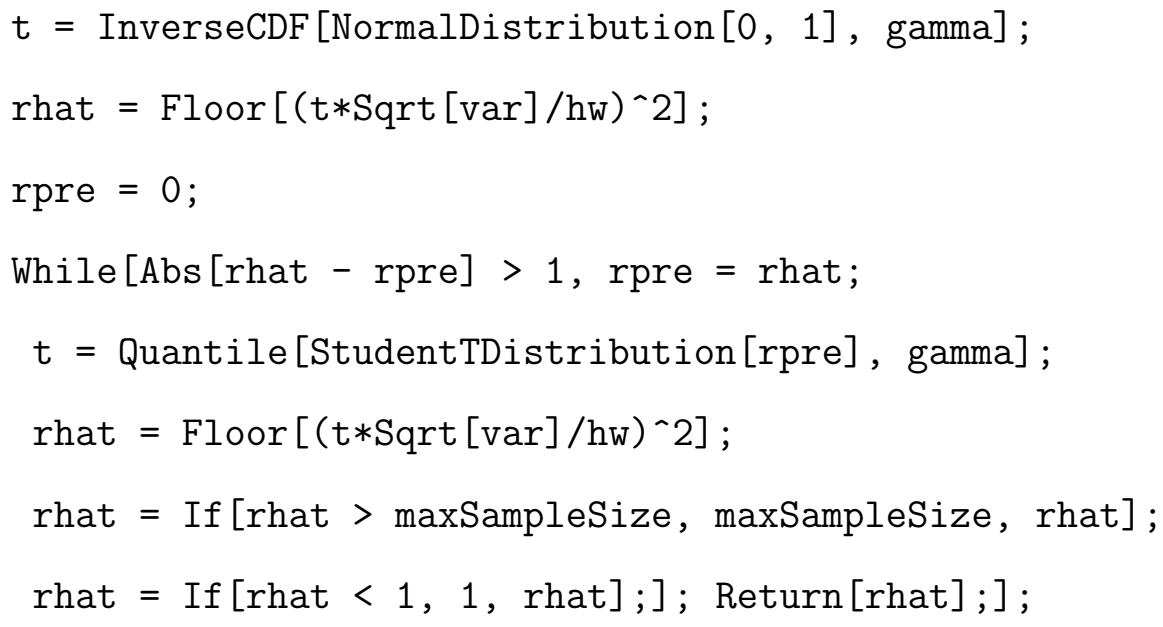

(*By Recursion *)

(*By Recursion *)

$(* \mathrm{~A}=(\backslash[\mathrm{Lambda}] 1+\backslash[\mathrm{Lambda}] 2) /(\backslash[\mathrm{Mu}] 1+\backslash[\mathrm{Mu}] 2) ; \backslash[\mathrm{Alpha}]=\backslash$ $\backslash[$ Lambda $] 2 /(\backslash[$ Lambda $] 1+\backslash[$ Lambda $] 2) ; x 0=\backslash[\mathrm{Mu}] 2 * \backslash[$ Lambda $] 1 /[\mathrm{Mu}] 1 ;$

*)

$(* \mathrm{~N} 1=100 ; \mathrm{g} 1=3 ;$

$\backslash[$ Lambda $] 1=40 ; \backslash[\mathrm{Mu}] 1=0.5 ; \backslash[\mathrm{Mu}] 2=0.5 ; *)$

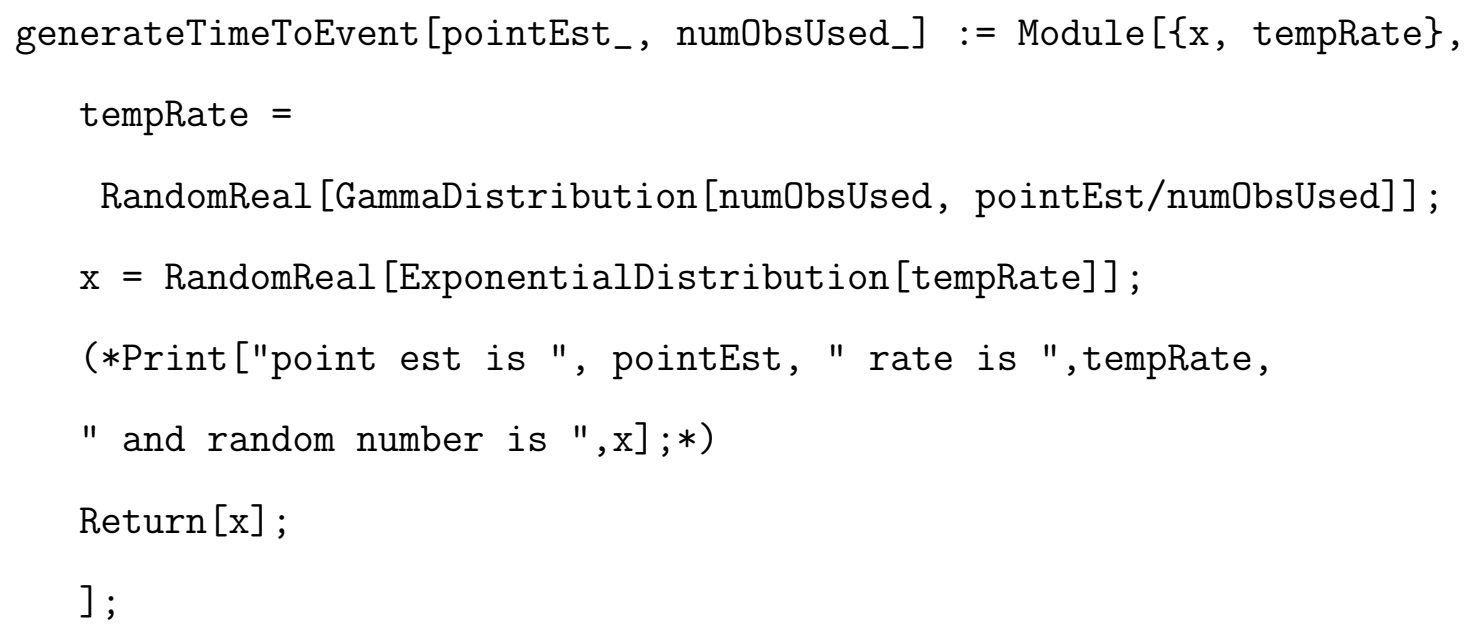


model2compsharedrepair [paramValuesArray_, paramSampleSizeArray_, outputFileName_] :=

Module [\{t, z, z1, z2, z3, sojourn1, sojourn2, sojourn0, ssavail, numstate0, numstate1, numstate2, temp, temp1, sortedssavail, combinedList, numAvails, CDFSSAvail, lambda, mu, lambdaSamples, muSamples\},

ssavail = Array $[, 2000]$;

lambda $=$ paramValuesArray $[[1]]$;

$\mathrm{mu}=\operatorname{paramValues} A \operatorname{rray}[[2]]$;

lambdaSamples = paramSampleSizeArray $[[1]]$;

muSamples = paramSampleSizeArray [[2]];

For $[i=1, i<=2000, i++, t=0 ; z=0$;

$z 1=0 ; z 2=0 ; z 3=0 ;$

sojourn2 = $0 ;$ sojourn $1=0 ;$ sojourn $0=0$;

numstate $2=0 ;$ numstate $1=0 ;$ numstate $0=0$;

(*lambda $=10$;

$\mathrm{mu}=50 ; *)$ While $[\mathrm{t}<1$, Label [state2];

$(* \operatorname{Pr}$ int ["state is $2 "] ; *)$ numstate 2 numstate $2+1$;

$z$ = generateTimeToEvent [2*lambda, lambdaSamples];

sojourn2 = sojourn2 $+z$;

(*Print["Total sojourn time in 2 ", sojourn2]; $*$ ) $t=t+z$;

$(* \operatorname{Print}[" \mathrm{t}$ is now ",t];*) If $[\mathrm{t}>1, \operatorname{Break}[]]$;

(*Now in state $1 *$ ) Label [state1];

$(*$ Print ["state is $1 "] ; *)$ numstate 1 = numstate $1+1$;

z1 = generateTimeToEvent [mu, muSamples $]$;

z2 = generateTimeToEvent [lambda, lambdaSamples];

If $[z 1<z 2$, numstate $2=$ numstate $2+1 ;$ 


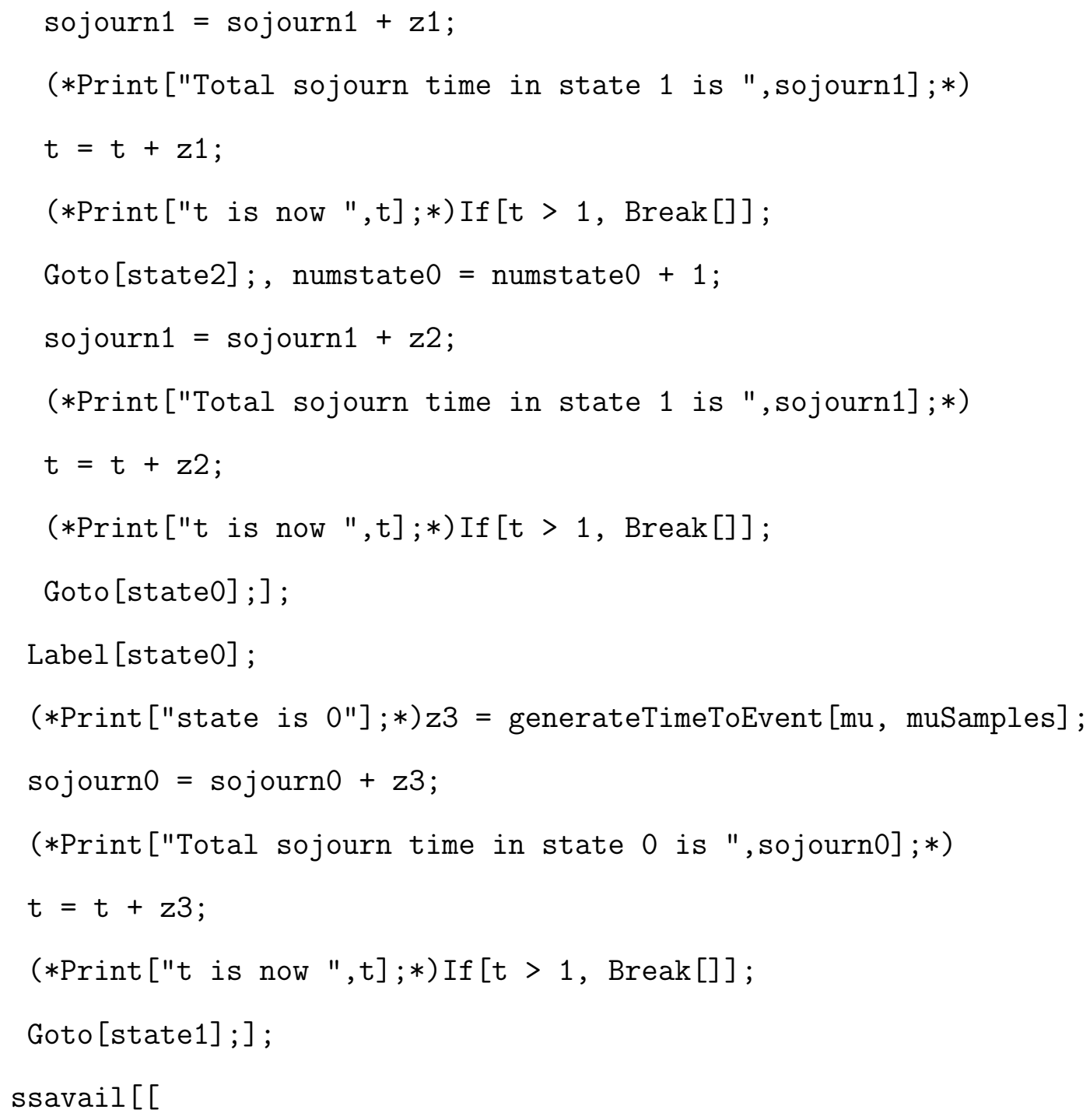




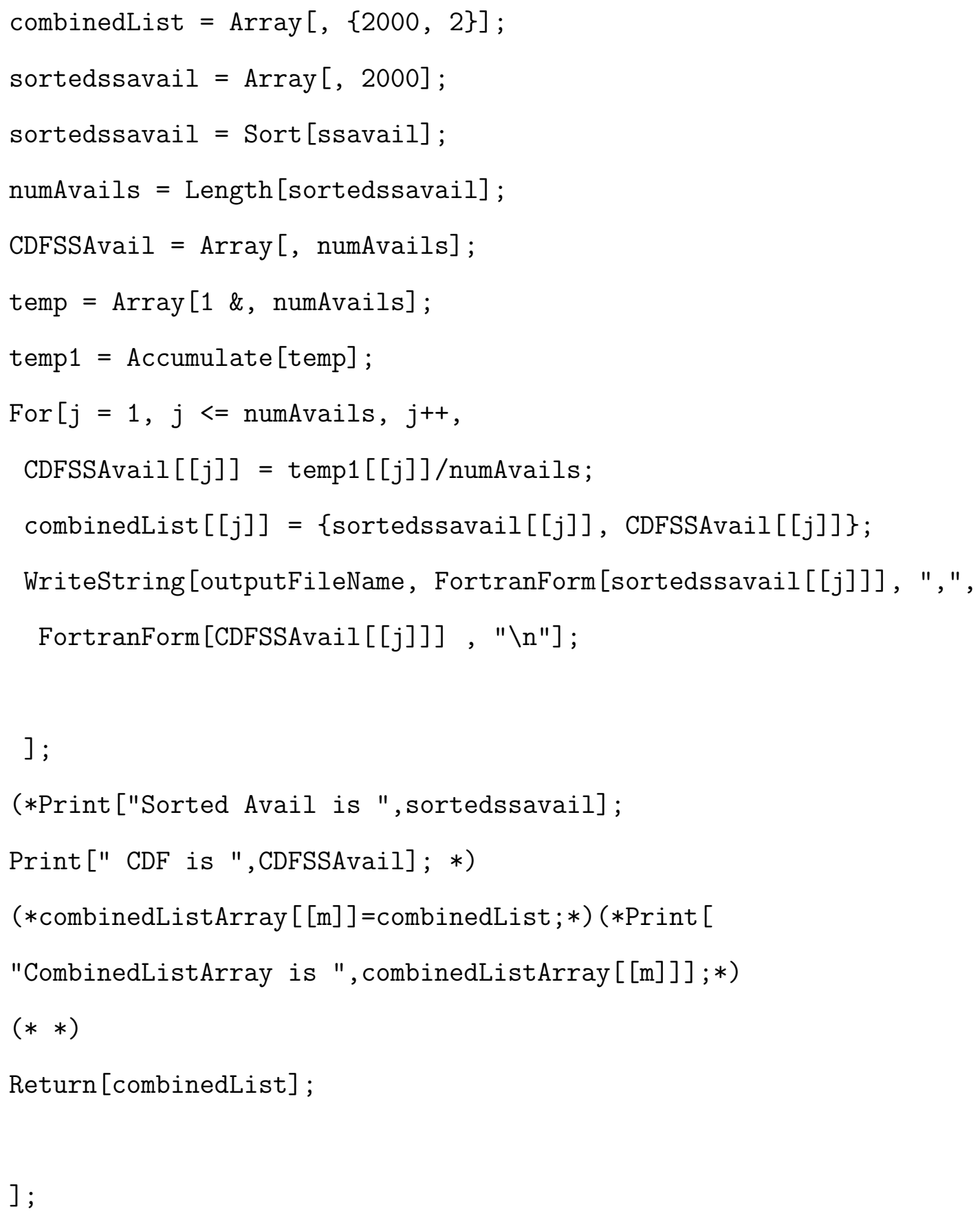




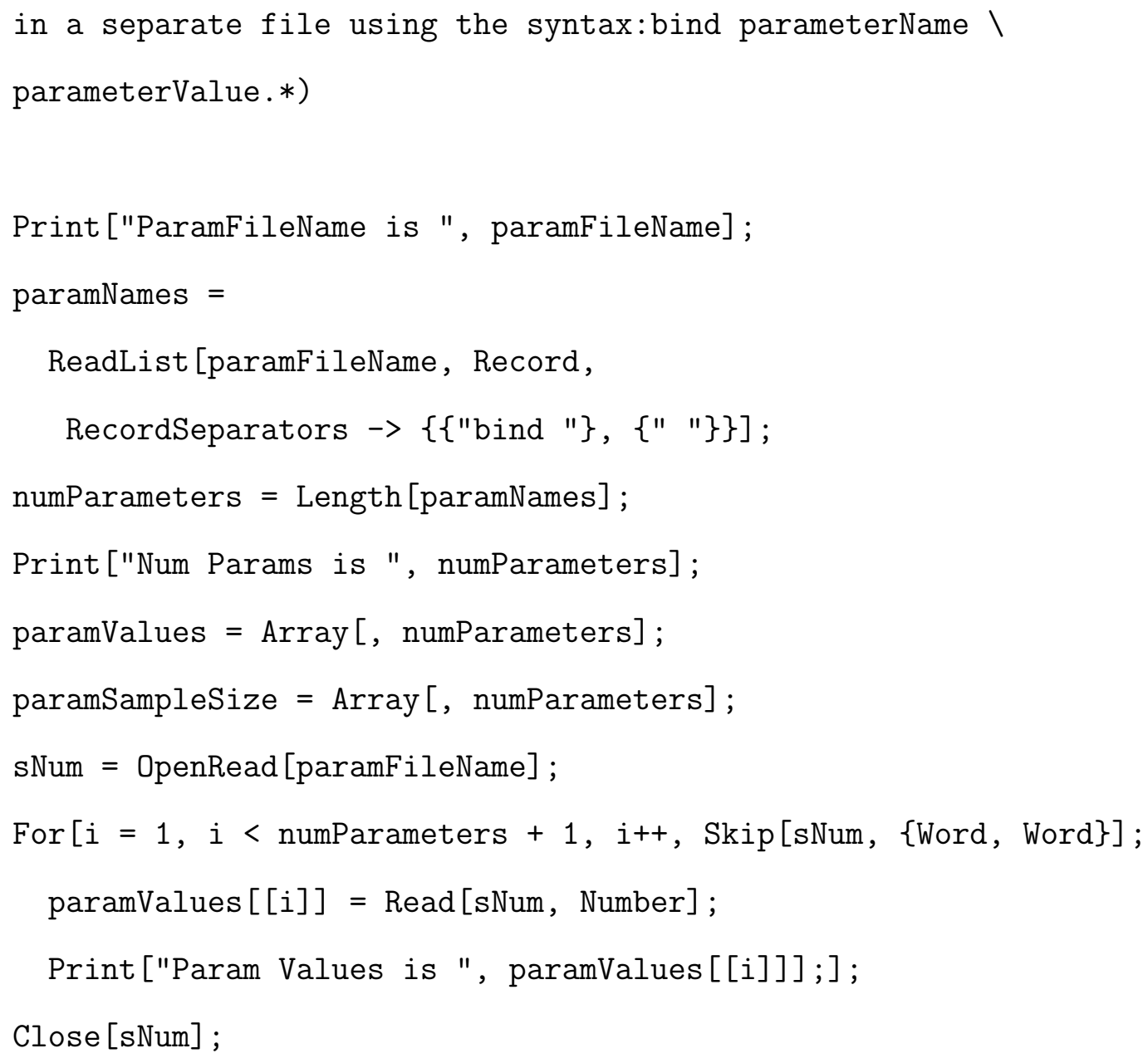




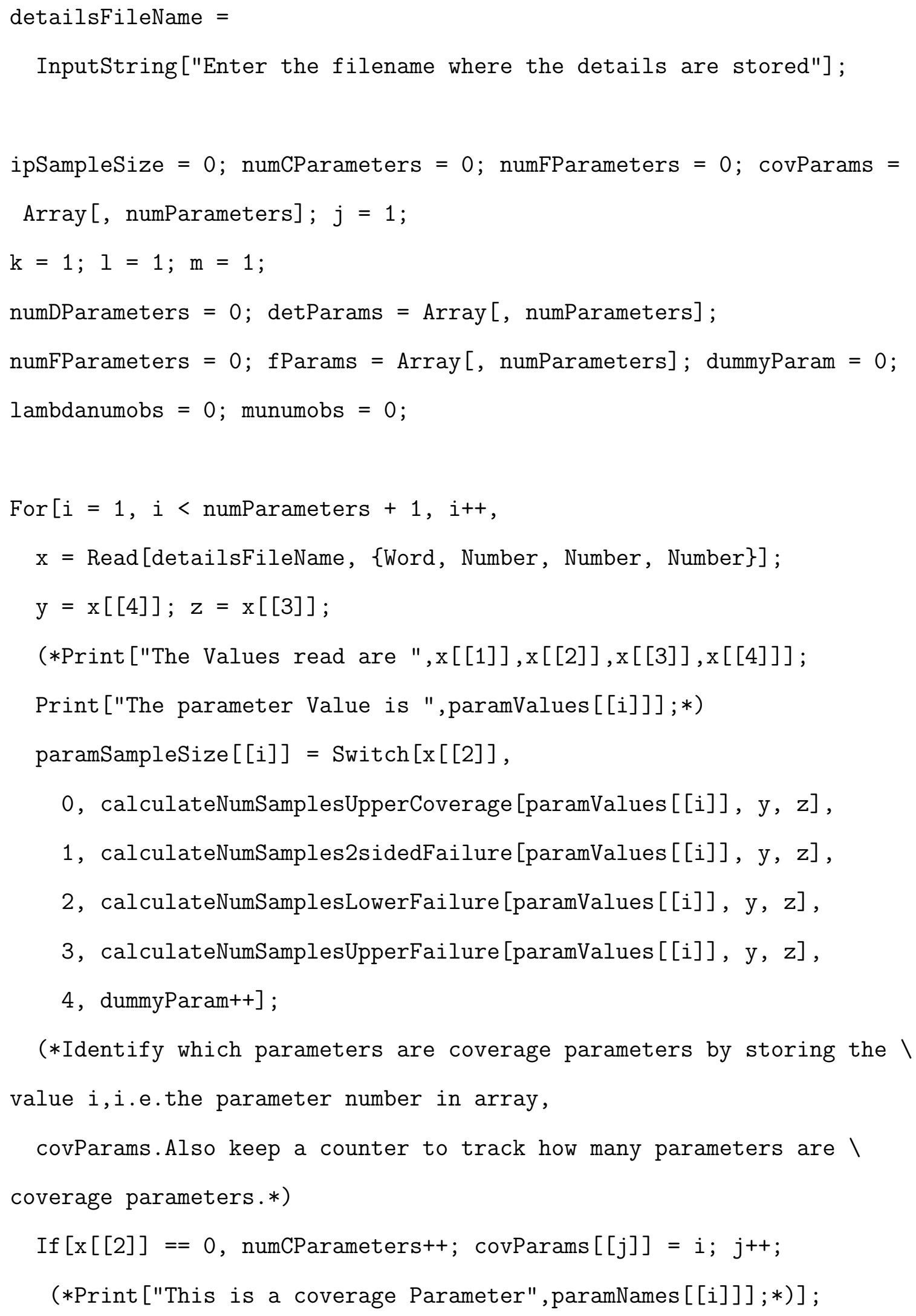




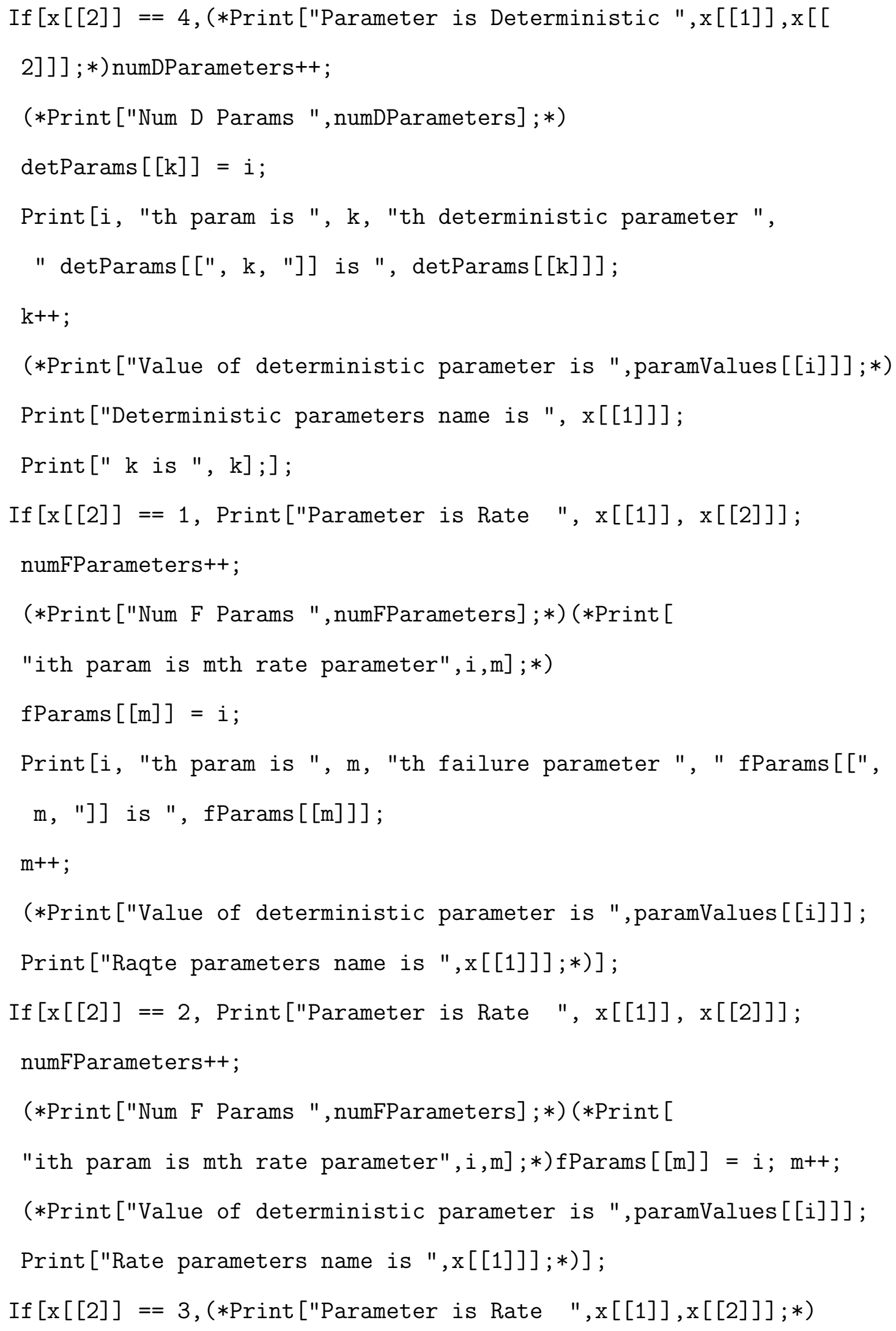




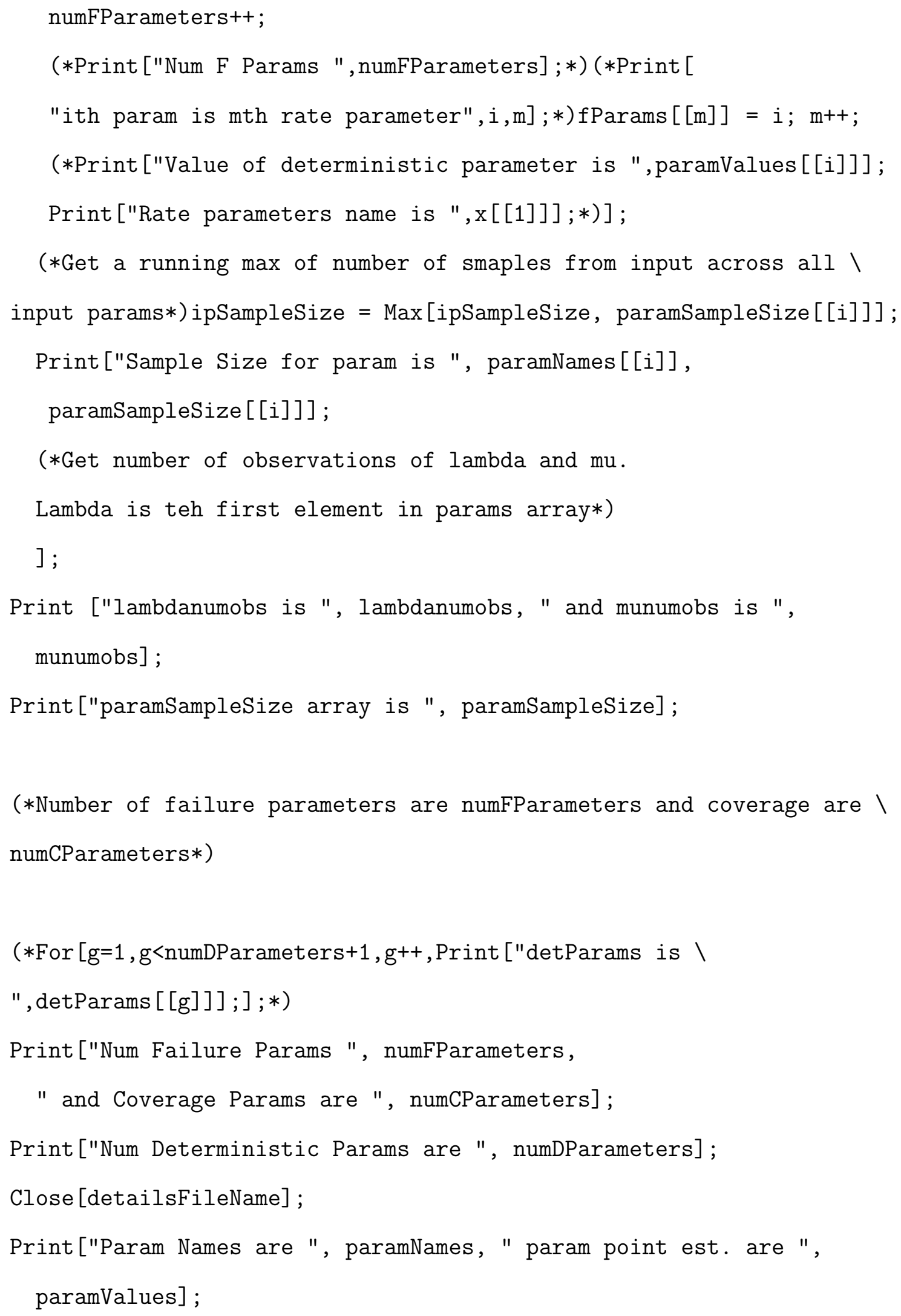


(*Since now we know which params are coverage, Store the coverage $\backslash$ parameter names, values and num samples in different arrays*)

(*parameterValue=Quantile [GammaDistribution [paramSampleSize $[[j]], \backslash$ paramValues $[[j]] /$ paramSampleSize $[[j]]]$, tempQuant $] *$ )

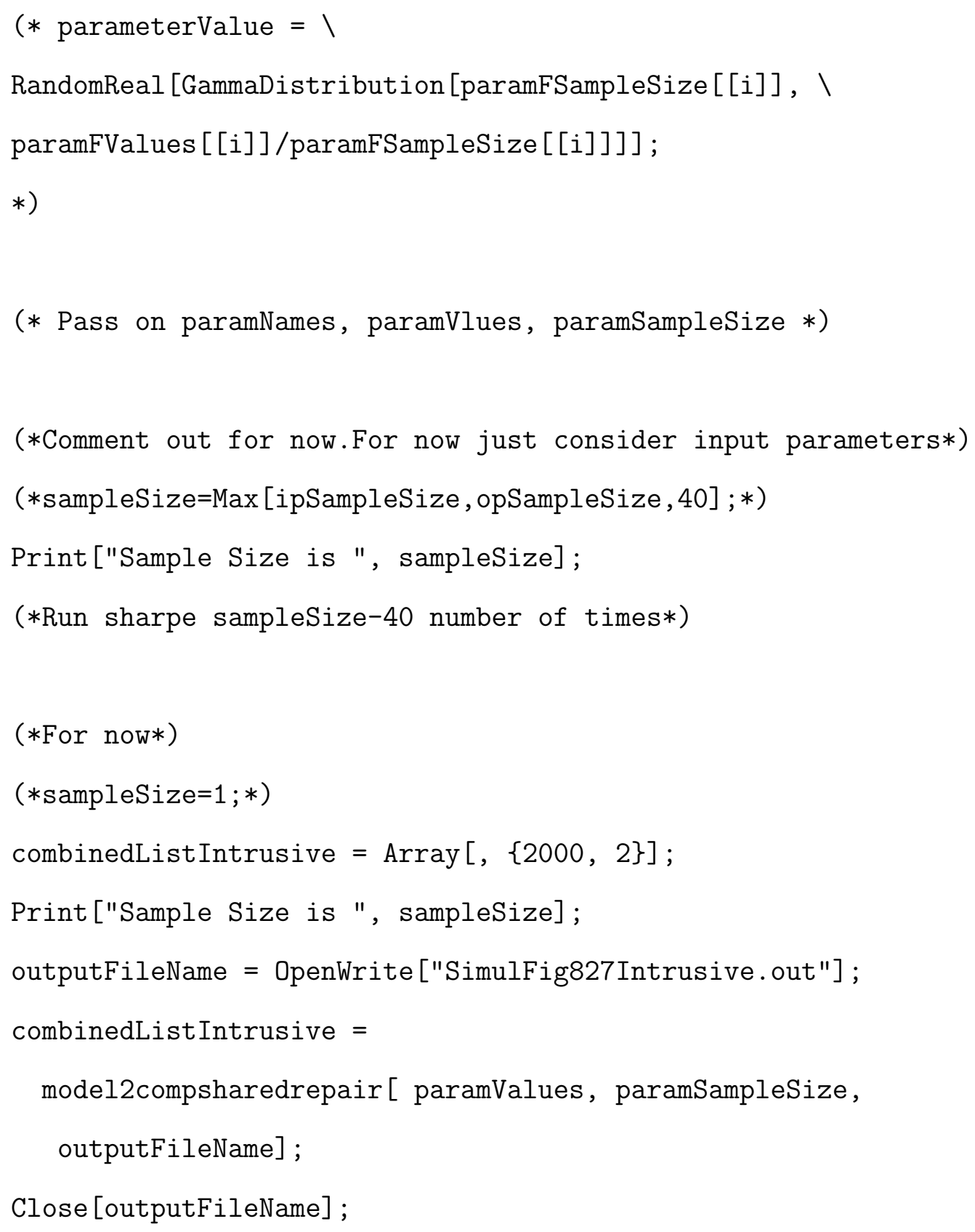




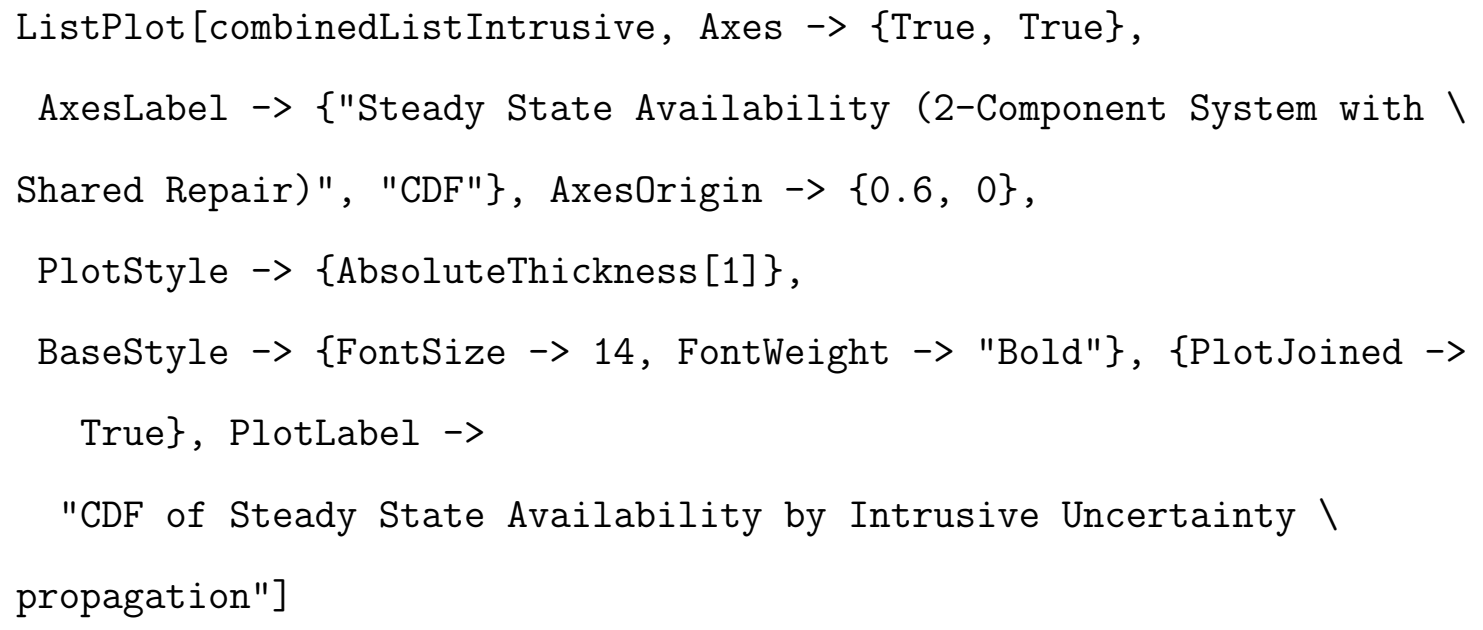




\section{Bibliography}

[1] Myrinet overview. In http://www.myri.com/myrinet/overview/.

[2] Wolfram mathematica 6, wolfram research, inc. In http://www.wolfram.com/products/mathematica/index.html.

[3] Oasis. In Specification: Business Process Execution Language for Web Services 1.1, 2004.

[4] M. Ajmone-Marsan, G. Balbo, G. Conte, S. Donatelli, and G. Franceschinis. Modeling with Generalized Stochastic Petri Nets. J. Wiley \& Sons, 1995.

[5] J.T. Blake, A.L. Reibman, and K.S. Trivedi. Sensitivity analysis of reliability and performability measures for multiprocessor systems. In 1988 ACM SIGMETRICS conf. on Measurement and modeling of computer systems.

[6] J.T. Blake and K.S. Trivedi. Reliability analysis of interconnection networks using hierarchical composition. IEEE Transactions on Reliability, 32:111-120, April 1989.

[7] A. Bobbio and K.S. Trivedi. An aggregation technique for the transient analysis of stiff markov chains. IEEE Transactions on Computers, C-35(9):803-814, September 1986.

[8] G. Bolch, K.S. Trivedi, S. Greiner, and H. de Meer. Queueing Networks and Markov Chains: Modeling and Performance Evaluation with Computer Science Applications. J. Wiley \& Sons, 2006.

[9] G. Box and M. Muller. A note on the generation of random normal deviates. In Annals Math. Statistics, volume 29, pages 610-611, 1958.

[10] D.B. Brown. A computerized algorithm for determining the reliability of redundant configurations. IEEE Transactions on Reliability, 20:121-124, 1971.

[11] R. Bryant. Graph based algorithms for boolean function manipulation. IEEE Transactions on Computers, 35(8):677-691, 1986. 
[12] J. Bukowski, L. Korn, and D. Wartenberg. Correlated inputs in quantitative risk assessment: The effects of distributional shape. Risk Analysis, 15(2):215-219, 1995.

[13] D. Chen, S. Dharmaraja, D-Y. Chen, L. Li, K.S. Trivedi, R. R. Some, and A.P. Nikora. Reliability and availability analysis for the jpl remore exploration and experimentation system. In In Proc. Int. Conf. on Dependable Systems and Networks, pages 337-344, 2002.

[14] H. Choi, V.G. Kulkarni, and K. S. Trivedi. Markov regenerative stochastic petri nets. Performance Evaluation, 20(1-3):337-357, 1994.

[15] G. Ciardo, A. Blakemore, P. F. Chimento, J. Muppala, and K. S. Trivedi. Automated generation and analysis of markov reward models using stochastic reward nets. IMA Volumes in Mathematics and its Applications, 48:145-191, 1993.

[16] D. W. Coit. System reliability confidence intervals for complex systems with estimated component reliability. IEEE Trans. on Reliability, 46(4):487-493, Dec 1997.

[17] Bert J. Debusschere, Habib N. Najm, Philippe P. Pébay, Omar M. Knio, Roger G. Ghanem, and Olivier P. Le Maître. Numerical challenges in the use of polynomial chaos representations for stochastic processes. SIAM J. Sci. Comput., 26:698-719, February 2005.

[18] A. Devaraj, K. Mishra, and K. Trivedi. Uncertainty propagation in analytic availability models. In Proc. of Symposium on Reliable Distributed Systems, SRDS 2010, pages 121-130, 2010.

[19] R. G. Easterling. Approximate confidence limits system reliability. Journal of the American Statistical Association, 67(337):220-222, Mar 1972.

[20] R. M. Fricks and K. S. Trivedi. Non-markovian dependability modeling. In Reliability and Maintainability Sysposium (RAMS), 2004.

[21] Ricardo M. Fricks and Michael Ketcham. Steady state availability estimation using field failure data. In Proc. Annual Reliability and Maintainability Symposium, Los Angeles, CA, January 2004.

[22] S. Garg, Y. Huang, C. Kintala, and K.S. Trivedi. Minimizing completion time of a program by checkpointing and rejuvenation. In SIGMETRICS, 1996.

[23] A. Gelman, J.B. Carlin, H.S. Stern, and D.B. Rubin. Bayesian Data Analysis. Chapman \& Hall, 2nd edition, 2003.

[24] R. German. Performance Analysis of Communication Systems: Modeling with Non-Markovian Stochastic Petri Nets. Kluwer Academic Publishers, 2000. 
[25] M. Grottke, A.P. Nikora, and K.S. Trivedi. An empirical investigation of fault types in space mission system software. In 40th Annual IEEE/IFIP Intl. Conf. on Dependable Systems and Networks (DSN 2010), 2010.

[26] M. Grottke and K.S. Trivedi. Fighting bugs: Remove, retry, replicate, and rejuvenate. IEEE Computer, 40(2):107-109, 2007.

[27] G. Haring, R. Marie, R. Puigjaner, and K. Trivedi. Loss formulas and their application to optimization for cellular networks. IEEE Transactions on Vehicular Technology, 50(3):664-673, May 2001.

[28] B.R. Haverkort and A.M.H. Meeuwissen. Sensitivity \& uncertainty analysis of markov-reward models. IEEE Trans. on Reliabiility, 44(1):147-154, March 1995.

[29] J. Helton and F. Davis. Latin hypercube sampling and propagation of uncertainty in analysis of complex systems. Reliability Engineering and System Safety, 81(1):23-69, 2003.

[30] J.C. Helton and F.J. Davis. Latin hypercube sampling and propagation of uncertainty in analysis of complex systems. Reliability Engineering and System Safety, 81(1):23-69, 2003.

[31] J. Hilllston. A Compositional Approach to Performance Modelling. Cambridge University Press, 1996.

[32] C. Hirel, B. Tuffin, and K.S. Trivedi. Spnp: Stochastic petri nets. version 6.0. In Computer Performance Evaluation.Modelling Techniques and Tools, volume 1786, pages 354-357. Springer Berlin / Heidelberg, 2000.

[33] O. Ibe, R. Howe, and K.S. Trivedi. Approximate availability analysis of vaxcluster systems. IEEE Trans. Reliiability, 38:146-152, 1989.

[34] O. Ibe, A. Sathaye, R. Howe, and K. S. Trivedi. Stochastic petri net modeling of vaxcluster availability. In Third Internaional Workshop on Petri Nets and Performance Models (PNPM89), pages 112-121, 1989.

[35] R.L. Iman and W.J. Conover. A distribution-free approach to inducing rank correlation among input variables. Communications in Statistics - Simulation and Computation, 11(3):311-334, 1982.

[36] B.W. Johnson. Design and analysis of fault-tolerant digital systems. AddisonWesley, 1989.

[37] M.E. Johnson. Multivariate Statistical Simulation. John Wiley \& Sons, New York, 1987. 
[38] R.E. Kass and L. Wasserman. The selection of prior distributions by formal rules. Journal of the American Statistical Association, 91(435):1343-1370, Sep. 1996.

[39] V.G. Kulkarni. Modeling and Analysis of Stochastic Systems. Chapman \& Hall, CRC, 1995.

[40] J.H. Lala and J.T. Sims. A dependability architecture framework for remote exploration \& experimentation computers. In The 29th International Symposium Fault-tolerant Computing (FTCS99), 1999.

[41] J. Laprie, J. Arlat, C. Beounes, and K. Kanoun. Architectural issues in software fault tolerance. In Software Fault Tolerance, M.Lyu (ed.). John Wiley, NY, 1994.

[42] G. J. Leiberman and S. M. Ross. Confidence intervals for independent exponential series systems. Journal of the American Statistical Association, 66(336):837840, Dec 1971.

[43] Y. Liu, K.S. Trivedi, Y. Ma, J. Han, and H. Levendel. Modeling and analysis of software rejuvenation in cable modem termination systems. In Proc. of the 13th Intl. Symp. on Software Reliability Engineering, ISSRE 2002, pages 159-170, 2002.

[44] D. Logothetis and K. S. Trivedi. The effect of detection and restoration times for error recovery in communication networks. Journal of Network and Systems Management, 5(2):145-191, 1997.

[45] D. Logothetis and K.S. Trivedi. Time-dependent behavior of redundant systems with deterministic repair. In 2nd International Workshop on the Numerical Solution of Markov Chains, pages 135-150, 1995.

[46] M.R. Lyu and V.B. Mendiratta. Software fault tolerance in a clustered architecture: techniques and reliability modeling. In 1999 IEEE Aerospace Conference.

[47] A. Madansky. Approximate confidence limits for the reliability of series and parallel systems. Technometrics, 7(4):495-503, Nov. 1965.

[48] M. Malhotra and K. S. Trivedi. Dependability modeling using petri net based models. IEEE Transactions on Reliability, 44(3):428-440, Sept. 1995.

[49] A.H. El Mawaziny and R.J. Buehler. Confidence limits for the reliability of series systems. Journal of the American Statistical Association, 62(320):14521459, Dec 1967.

[50] M.D. McKay, R.J. Beckman, and W.J. Conover. A comparison of three methods for selecting values of input variables in the analysis of output from a computer code. Technometrics, 42:55-61, 2000. 
[51] S.J. Mildenhall. Correlation and aggregate loss distributions with an emphasis on the iman-conover method. In CAS Working Party on Correlation. Casualty Actuarial Society Forum, Winter 2005.

[52] K. Mishra and K. S. Trivedi. Uncertainty analysis of reliability of the remote exploration and experimentation system. (In preparation for) Journal of Spacecrafts and Rockets.

[53] K. Mishra and K. S. Trivedi. Uncertainty propagation through software dependability models. In (To Appear In) The 22nd annual International Symposium on Software Reliability Engineering (ISSRE 2011).

[54] K. Mishra and K. S. Trivedi. An unobtrusive method for uncertainty propagation in stochastic dependability models. International Journal of Reliability and Quality Performance (IJRQP). (To appear in), Dec. 2011.

[55] K. Mishra and K.S. Trivedi. A non-obtrusive method for uncertainty propagation in analytic dependability models. In In Proc. 4th Asia-Pacific Intl. Symp. on Advanced Reliability and Maintenance Modeling (APARM 2010), 2010.

[56] J. Muppala, R. Fricks, and K.S. Trivedi. Techniques for system dependability evaluation. In Computational Probability, W. Grassman (ed.), pages 445-480. Kluwer Academic Publishers, 2000.

[57] M.J. Nauta. Separation of uncertainty and variability in quantitative microbial risk assessment models. International Journal of Food Microbiology, 57:9-18, 2000 .

[58] Y.W. Ng and A.A. Avizienis. A unified reliability model for fault-tolerant computers. IEEE Trans. on Computers, C-29(211):1002-1011, 1980.

[59] W. L. Oberkampf, S. M. DeLand, B. M. Rutherford, K. V. Diegert, and K. F. Alvin. Estimation of total uncertainty in modeling and simulation. SANDIA REPORT, SAND2000-0824, pages 698-719, April 2000.

[60] A.V. Oppenheim, R. W. Schafer, and J. R. Buck. Discrete-time signal processing. Prentice Hall, 1999.

[61] J. Pukite and P. Pukite. Modeling for Reliability Analysis : Markov Modeling for Reliability, Maintainability, Safety, and Supportability Analyses of Complex Systems. John Wiley and Sons, 1998.

[62] A. Puliafito, M. Scarpa, and K. S. Trivedi. Petri nets with k-simultaneously enabled generally distributed timed transitions. Performance Evaluation, 32(1):134, Feb. 1998. 
[63] P. Rabinowitz and P. J. Davis. Methods of Numerical Integration. Dover Publications, 2007.

[64] S. Rai, M. Veeraraghavan, and K. S. Trivedi. A survey of efficient reliability computation using disjoint products approach. Networks, 25:147-163, 1995.

[65] A.V. Ramesh and K.S. Trivedi. On the sensitivity of transient solutions of markov models. In 1993 ACM SIGMETRICS conf. on Measurement and modeling of computer systems.

[66] K.D. Rao, H.S. Kushwahaa, A.K. Verma, and A. Srividya. Quantification of epistemic and aleatory uncertainties in level-1 probabilistic safety assessment studies. Reliability Engineering and System Safety, 92:947-956, 2007.

[67] D. Rasch. Sample size determination for estimating the parameter of an exponential distribution. Akademie der Landwirtacheftswissenschaften der DDR Forschungszentrurn fir Tierproduktion Dummerstorf-Rostock, 19:521$528,1977$.

[68] D.A. Rennels, D.W. Caldwell, R. Hwang, and K. Mesarina. A fault-tolerant embedded microcontroller testbed. In Pacific Rim Int. Symp. Fault Tolerant Systems (PRFTS97), 1997.

[69] J.A. Rohr. Starex self-repair routines: software recovery in the jpl-star computer. In The 25th Int. Symp. Fault-tolerant Computing, 1995.

[70] J.A. Rohr. Software-implemented fault tolerance for supercomputing in space. In The 28th Int. Symp. Fault-Tolerant Computing, 1998.

[71] A. Roy, D.S. Kim, and K.S. Trivedi. Attack countermeasure trees (act): towards unifying the constructs of attack and defense trees. Security and Communication Networks, 2011.

[72] Robin A. Sahner, K.S. Trivedi, and A. Puliafito. Performance and Reliability Analysis of Computer Systems: An Example-Based Approach Using the SHARPE Software Package. J. Wiley \& Sons, 1996.

[73] R.N. Sanga, S.M. Bartell, R.A. Ponce, A.A.P. Boischio, C.R. Joiris, C.H. Pierce, and E.M. Faustman. Effects of uncertainties on exposure estimates to methylmercury: A monte carlo analysis of exposure biomarkers versus dietary recall estimation. Risk Analysis, 21(5):859-868, 2001.

[74] T. K. Sarkar. An exact lower confidence bound for the reliability of a series system where each component has an exponential time to failure. Technometrics, 13(3):535-546, Aug 1971. 
[75] N. Sato and K.S. Trivedi. Stochastic modeling of composite web services for closed-form analysis of their performance and reliability bottlenecks. In 5th. Intl. Conf. on Service Oriented Computing, ICSOC, 2007.

[76] A. Satyanarayana and M. K. Chang. Network reliability and the factoring theorem. Networks, 13:107-120, 1983.

[77] A. Satyanarayana and A. Prabhakar. New toplogogical formula and rapid algorithm for reliability analysis of complex networks. IEEE Transactions on Reliability, 7:82-100, 1978.

[78] S.Garg, A. Puliafito, M. Telek, and K. S. Trivedi. Analysis of software rejuvenation using markov regenerative stochastic petri net. In International Symposium on Software Reliability Engineering (ISSRE), 1995.

[79] D.P. Siewiorek and R. S. Swarz. Reliable Computer Systems: Design and Evaluation. AK Peters, Ltd, 1998.

[80] N. D. Singpurwalla. Reliability and Risk: A Bayesian Perspective. John Wiley \& Sons, 1 edition, 2006.

[81] A.E. Smith, P.B. Ryan, and J.S. Evans. The effect of neglection correlations when propagating uncertainty and estimating the population distribution risk. Risk Analysis, 12(4):467-474, 1992.

[82] W.E. Smith, K.S. Trivedi, L.A. Tomek, and J. Ackaret. Availability analysis of blade server systems. IBM Systems Journal, 47(4), 2008.

[83] M. Stamatelatos, G. Apostolakis, H. Dezfuli, C. Everline, S. Guarro, P. Moeini, A Mosleh, T. Paulos, and R. Youngblood. Probabilistic risk assessment procedures guide for nasa managers and practitioners. http://www.hq.nasa.gov/office/codeq/doctree/praguide.pdf, Ver. 1.1, 2002.

[84] L.A. Tomek and K.S. Trivedi. Fixed point iteration in availability modeling. In 5th Intl. GI/ITG/GMA Conf. on Fault-Tolerant Computing Systems, pages 229-240, 1991.

[85] K. Trivedi, D. Wang, D.J. Hunt, A. Rindos, W.E. Smith, and B. Vashaw. Availability modeling of sip protocol on ibm websphere. In Proc. of Pacific Rim Dependability Conference, 2008.

[86] K.S. Trivedi. Probability and Statistics with Reliability, Queuing and Computer Science Applications. J. Wiley \& Sons, New York, 2001.

[87] K.S. Trivedi and R. Sahner. Sharpe at the age of twenty two. SIGMETRICS Performance Evauation Review, 36(4):52-57, 2009. 
[88] J. von Neumann. Various techniques used in connection with random digits. monte carlo methods. In Nat. Bureau Standards, volume 12, page 3638, 1951.

[89] D. Wang. Service reliability: Models, algorithms and applications. Ph.D. dissertation, Department of Computer Science, Duke University, 2007.

[90] K. Whisnant, R. K. Iyer, P. Jones, R. R. Some, and D. A. Rennels. An experimental evaluation of the ree sift environment for spaceborne applications. In International Conference on Dependable Systems and Networks (DSN 2002), pages 585-594, 2002.

[91] F.-C. Wu and Y.P. Tsang. Second-order monte carlo uncertainty/variability analysis using correlated model parameters: application to salmonid embryo survival risk assessment. Ecological Modeling, 177:393-414, 2004.

[92] W. Xie, H. Sun, Y. Cao, and K. S. Trivedi. Modeling of user perceived web-server availability. In 38th annual IEEE International Conference on Communications, May 2003.

[93] L. Yin, M. Smith, and K.S. Trivedi. Uncertainty analysis in reliability modeling. Reliability and Maintainability Symposium, pages 229-234, 2001. 


\section{Biography}

Kesari Mishra was born in Darbhanga, in the state of Bihar, India. He received his B.Tech. degree in Electronics and Communication Engineering from Indian Institute of Technology, Roorkee, in 2001 and his M.S. degree in Electrical and Computer Engineering from Duke University in 2004. He worked as a graduate intern at Sun Microsystems Inc in the summers of 2002 and 2003. He is currently working at NetApp Inc (since 2004), as a member of technical staff in the Performance Engineering group. His research interests include uncertainty propagation and quantification, statistical inference and performance modeling. His scientific publications are listed below.

\section{Publications}

- K. Mishra and K. S. Trivedi. "Uncertainty Propagation through Software Dependability Models", To appear in the 22nd Annual International Symposium on Software Reliability Engineering (ISSRE 2011).

- K. Mishra and K. S. Trivedi. "An Unobtrusive Method for Uncertainty Propagation in Stochastic Dependability Models". To appear in International Journal of Reliability and Quality Performance (IJRQP), December 2011.

- K. Mishra and K. S. Trivedi. "Uncertainty Analysis of Reliability of the Remote Exploration and Experimentation System". Accepted by Journal of Spacecrafts and Rockets. 
- A. Devaraj and K. Mishra and K. Trivedi. "Uncertainty Propagation in Analytic Availability Models". In 29th IEEE Symposium on Reliable Distributed Systems (SRDS), pp. 121-130, Delhi, 2010.

- K. Mishra. "Statistics of Quantitative Availability Assurance". Student paper in 39th Annual IEEE International Conference on Dependable Systems and Networks (DSN), 2009.

- K. Mishra and K. Trivedi. "Model Based Approach for Autonomic Availability Management". In International Service Availability Symposium (ISAS), pp. 116, Helsinki, 2006.

\section{Patents}

- K. Mishra and S. Ramany. "Load Balancing a Data Storage System". United States Patent No. 7, 669, 029. Issued in Feb. 2010.

- D. Sawyer and K. Mishra and S. Ramany. "Load Balanced Storage Provisioning". United States Patent No. 7, 849, 180. Issued in Dec. 2010. 